\title{
Temporal and geographical patterns of solid waste collected at recycling centres
}

\author{
Edjabou, Maklawe Essonanawe; Faraca, Giorgia; Boldrin, Alessio; Astrup, Thomas Fruergaard
}

Published in:

Journal of Environmental Management

Link to article, DOI:

10.1016/j.jenvman.2019.05.059

Publication date:

2019

Document Version

Peer reviewed version

Link back to DTU Orbit

Citation (APA):

Edjabou, M. E., Faraca, G., Boldrin, A., \& Astrup, T. F. (2019). Temporal and geographical patterns of solid waste collected at recycling centres. Journal of Environmental Management, 245, 384-397.

https://doi.org/10.1016/j.jenvman.2019.05.059

\section{General rights}

Copyright and moral rights for the publications made accessible in the public portal are retained by the authors and/or other copyright owners and it is a condition of accessing publications that users recognise and abide by the legal requirements associated with these rights.

- Users may download and print one copy of any publication from the public portal for the purpose of private study or research.

- You may not further distribute the material or use it for any profit-making activity or commercial gain

- You may freely distribute the URL identifying the publication in the public portal 


\section{Temporal and geographical patterns of solid waste collected at recycling centres}

Maklawe Essonanawe Edjabou*, Giorgia Faraca, Alessio Boldrin, and Thomas Fruergaard
Astrup

Department of Environmental Engineering, Technical University of Denmark, $2800 \mathrm{Kgs}$.

Lyngby, Denmark

*) Corresponding author: vine@env.dtu.dk;

Phone number: +45 45251498 


\section{Abstract}

3 Citizens increasingly dispose their waste at household waste recycling centres (HWRC). To

4 enhance the collection of recyclables materials, local authorities and waste management

5 companies invest considerable resources in planning. While the planning of these centres

6 requires a comprehensive understanding of collected solid waste, only limited studies have

7 consistently investigated waste data from HWRC. To fill this knowledge gap, historical data for

8 HWRC from the Greater Copenhagen, Central Zealand, Silkeborg and Djursland in Denmark

9 were analysed with regards to temporal and geographical variation. The results showed the mass

10 of collected waste varies seasonally; this trend was consistently seen during the period 2010 -

11 2016. Moreover, the data revealed that the total waste collected was principally driven by the

12 number of visitors. The geometric bar plot and ternary plot depicted an increase in the percentage

13 of recyclable materials, whereas the percentage of incinerated waste decreased during the period

14 2010-2016. The waste characterisation study indicated that about $7 \%$ of small miscellaneous

15 combustible waste was brought in black plastic bag, although these bags were forbidden; the

16 results suggest that the percentage of misplaced recyclable materials could considerably decrease

17 if citizens bring their waste in clear plastic bags. 
20 Key words:

21 Household waste recycling centres;

22 Mean ratio

23 Ternary plot

24 clr (centered log-ratio)-biplot

25 Seasonality 


\section{Introduction}

Household waste is generally collected in a full service scheme in many developed

28 countries. In recent year, more and more citizens dispose their waste such as bulky, small

29 miscellaneous and recyclable waste directly at household waste recycling centres (HWRC)

30 (WRAP, 2012). For example, it was estimated that in Denmark about 25\% of the total household

31 waste was collected at HWRC in 2015 (Toft et al., 2017). Given this growing interest, various

32 initiatives aiming to increase recycling rates at these centres were established, including

33 competitive salaries, education and training of staffs, public information campaigns, appealing

34 signage, effective sign posting, good site layout, and extended opening hours (Krook and

35 Eklund, 2010; Ordoñez et al., 2014, and WRAP,2012). In Denmark, for example, an increasing

36 number of HWRC is open during 24 hours (a day), enabling citizens to dispose of their waste at

37 any time. Further measures towards increasing recycling focus on reducing the residual waste

38 fraction, which in Denmark is denominated "small miscellaneous combustible". For example, a

39 black bag policy was enforced in Denmark in 2010, prohibiting citizens to handle solid waste in

40 a black plastic bag at the HWRC. Instead, site users must handle their waste in clear plastic bags

41 to enable the staff to check whether the bags contain recyclable materials that could be further

42 sorted prior to the disposal of the bag (Danish EPA, 2018).

43 Although the planning and implementation of different initiatives are very costly, both

44 monitoring of their possible effect on recycling rates as well as documentation of the lessons

45 learnt at the local level are quite scarce. This may be explained by two factors. First, the lack of

46 data on waste collected at HWRC, despite their important contribution to the total municipal

47 solid waste. For example, European solid waste statistics publish only aggregated data

48 (European Commission, 2016). While waste collected at HWRC in Norway (Avfall Norge,

49 2019) and Sweden (RVF, 2018) are published each year, these data are not available for 
50 Denmark. Moreover, the existing data for these Nordic countries are solely the total mass

51 collected. As an example, the yearly waste collected at HWRC are available for all regions and

52 main cities from 2015 to 2018 (Avfall Norge, 2019). However, data describing seasonal patterns

53 remain sparse despite their importance. Second, existing methods applied to waste composition

54 datasets (in percentage) suffer from some serious shortcomings. Statistical analysis applied to

55 compare waste fractional composition datasets (in percentage) in order to assess the

56 effectiveness of an implemented measurement does not consider the inherent nature of

57 compositional dataset (Katsamaki et al., 1998; Navarro-Esbrí et al., 2002). The waste fractional

58 composition dataset is a "closed dataset" (Edjabou et al., 2017a), because the percentage of

59 individual waste fractions must sum to 100, which is known as the "total sum constrain"

60 problem (Aitchison, 1994; Buccianti and Pawlowsky-Glahn, 2011). Consequently, statistical

61 analysis applied directly to a waste fractional composition dataset results in inaccurate outcome

62 such as spurious correlation coefficients (Edjabou et al., 2017b). Moreover, although the waste

63 composition is derived from mass, previously published studies have found no relationship

64 between the distribution of the two datasets: (1) waste fraction composition in percentage and

65 (2) waste fractional generation rate such kg/person/week (Edjabou et al., 2018; Edjabou et al.,

66 2017b). As a solution, compositional data analysis technique (Aitchison, 1994; Pawlowsky-

67 Glahn et al., 2015) was shown to be a valid method to adequately analyse waste fractional

68 composition data (Edjabou et al., 2017b). This means that, to provide a complete interpretation

69 of statistical analysis where total waste is included, waste data could be modelled as

70 compositional data with total sum (Kynčlová et al., 2015).

71 Two issues are related to the inherent structure of waste composition. Firstly, a study of a

72 single waste fraction may provide incomplete knowledge of the whole system, given that waste 
management systems are generally "closed systems” (Martinez-Sanchez et al., 2016). Secondly,

74 the dynamic nature of waste may mislead the estimates of recycling rates. A typical example is

75 "heavy" waste fractions such as garden waste and construction and demolition (C\&D). The

76 collected amount of these fractions is mainly subject to the vagaries of the weather (Boldrin and

77 Christensen, 2010) and economic growth (Danish Government, 2013), respectively. These

78 inherent dynamics characteristics of waste collected at HWRC require a critical method to

79 reveal and document the impact of any changes in recycling rates at the HWRC.

80 To fill the knowledge gap aforementioned, this study aims at investigating geographical and

81 temporal patterns in the generation and composition of solid waste from HWRC. This is done

82 by: (i) collecting and analysing historical waste data of 36 HWRCs grouped into four study

83 areas; (ii) statistically comparing different variables, including the number of visitors,

84 population, generation and composition of solid waste; (iii) developing dynamic visualization

85 tools to ease the interpretation and communication of results; (iv) assessing the effectiveness of

86 the black bag policy at HWRCs in Greater Copenhagen, Central Zealand and Silkeborg. The

87 findings could support local authorities in improving the management and planning of HWRCs.

\section{$88 \quad 2$ Methods and materials}

$89 \quad 2.1$ System definition

\section{$90 \quad$ 2.1.1 Waste fractions classification}

91 Waste fractions were grouped into two compositional datasets, as presented in Table 1:

92 (1) eleven main waste fractions: (i) bulky, (ii) small miscellaneous combustible (SMC), (iii)

93 construction and demolition waste (C\&D), (iv) garden waste, (v) soil, (vi) fibres and other, (vii)

94 glass, (viii) metal, (ix) plastic, (x) wood and (xi) other waste (OW). Construction and demolition

95 (C\&D) waste comprises of bricks, blocks, plaster, concrete, tiles, paving slabs, etc. The target

96 waste fractions are included in the European Union's circular economy package and the Danish 
97 Resource Strategy Plan (Danish Government, 2013). They consist of (a) fibres and others

98 (paper, board, and textile), (b) metal, (c) glass, (d) wood and (f) plastics. Due to difference in

99 number and type of waste fractions in HWRCs, we first aggregated all fractions into detailed

100 material fractions (Table 1). These fractions were subsequently grouped into the main fractions.

101 For example, we grouped textile, paper and board into "fibres and other" for consistency reasons

102 and because textile was poorly recorded before 2016.

103 (2) waste treatment forms consist of (i) incineration (ii) recycling, and (iii) other treatments

104 (European Commission, 2016; Toft et al., 2017). This classification enables to assess the

105 progress toward waste recycling targets based on collected waste from HWRCs. Recyclable are

106 materials fractions intended for recycling such as garden waste, paper, board, glass, etc. Thus,

107 "recycling rates" refers to the percentage of waste sorted at HWRC for recycling, whereas the 108 potential recycling rate is the percentage of recyclable after adding misplaced materials. Other

109 treatments involve temporary storage, landfill and special treatments (Bigum et al., 2013). Waste

110 fractions that are neither "recyclable" nor suitable for other treatments are incinerated. Often,

111 these waste fractions are collected as "miscellaneous combustible" and are generally subdivided

112 into "small miscellaneous combustible (SMC)" and "bulky" waste. While small miscellaneous

113 combustible comprises of small items, such as dirty paper and board, composite materials, etc.,

114 bulky waste is items larger than 50x50x100 cm (Nilsson and Christensen, 2010). It includes for 115 example furniture, mattress, bric-a-brac, homewares, etc.

\section{$116 \quad$ 2.1.2 Study area and household waste recycling centres}

117 This study involved 36 HWRCs from 13 municipalities (Figure 1), located in the areas

118 ranging from densely (blue on the map) to sparsely populated (North Djursland municipality)

119 municipalities. 
HWRCs were grouped into Zealand consisting to Greater Copenhagen, Central Zealand,

121 and Jutland, which comprises of Silkeborg and Djursland (Figure 1). The HWRCs from each

122 region are managed by a waste company owned by a consortium of municipalities in this region.

123 One of the differences between these regions is the ratio between housing types, namely single-

124 family and multi-family houses (Table S1). Here, single-family houses have a garden, while

125 multi-family houses are block apartments without a garden. The highest percentage of single126 family houses was 97\% (municipality of Lejre in Central Zealand) and the lowest was 3\%

127 (municipality of Frederiksberg in Greater Copenhagen). In the contrary, the percentage of

128 single-family houses was $78 \%$ in the municipality of Dragoer that is also located in the capital

129 city (Greater Copenhagen). Overall, the percentage of single-family houses ranges from 62-94\%

130 in Djursland, Silkeborg and Central Zealand (Table S1). The distribution between housing types

131 remains stable during the period 2010-2016 (Statistics Denmark, 2017).

132 Although the management of HWRCs follows the national waste regulations and 133 legislations, each municipality and/or waste management company can organise their HWRCs 134 according to local conditions. This involves, for example, measures to increase recycling rates, 135 such as opening hours, number of waste fractions, employees at HWRCs, signage, billing 136 system, etc.

\section{$137 \quad$ 2.1.3 Household waste management}

138 The waste collection system in this study consisted of (1) door-to-door waste collection for 139 residual household waste, source-separated recyclables materials (paper, cardboard, metal and 140 glass), bulky waste (Edjabou et al., 2015); (2) public collection points for glass and paper, and 141 (3) HWRCs.

142 In single-family residential areas, households have their own waste bins; whereas in the 
143 multi-family residential areas several households shared bins (join full service collection point).

144 Collection frequencies for residual waste depended on housing types. It was every two 145 weeks in single-family residential areas and every week in multi-family areas. However, source-

146 sorted recyclable and bulky waste were collected upon request or on monthly basis.

147 Waste collected at HWRCs has considerably increased from 10 to about $30-54 \%$ of 148 households waste in this study area (Danish Government, 2013).

$149 \quad 2.2$ Data collection

150 We used data on (1) mass of collected individual waste material fractions, (2) composition 151 of small miscellaneous combustible, (3) number of visitors, (4) population, and (5) housing 152 types.

\section{$153 \quad$ 2.2.1 Mass of collected waste, number of visitors, population, and housing types}

154 Waste management companies provided monthly amount of individual waste fractions 155 collected and the number of sites users. The waste collection rates (WCR) were then computed 156 for each municipality as (1) per household (WCR house [kg/household/year]), (2) per inhabitant

157 (WCR inhabitant $_{\text {t }}[\mathrm{kg} / \mathrm{inh}$ abitant/year $\left.]\right)$, and (3) per visitor $\left(\mathrm{WCR}_{\text {visitor }}[\mathrm{kg} /\right.$ visitors/year $\left.]\right)$. This 158 method enables to consistently compared HWRC.

159 We excluded data for waste directly reused at the site through "intern" flea market and 160 "Waste exchange centres" (a visitor can leave reusable items that others can take away), because 161 HWRCs did not consistently registered these waste flows during the period 2010-2016. 162 Similarly, materials such as good quality furniture or items given to charity were not included in 163 this study.

164 The number of site users includes all visitors entering HWRC by any means (pedestrian, 165 bicycles, cars, van, etc...). Given the diversity of counting systems and discrepancy in their 
166 reliability (Cope et al., 1999), we excluded uncertainties associated with counting systems. Data

167 for number of households and inhabitant were retrieved from the Danish national statistics

168 database (Statistics Denmark, 2017).

$169 \quad$ 2.2.3 Composition of small miscellaneous combustible (SMC)

170 Small miscellaneous combustible (SMC) from seven HWRCs (Figure 1) was characterised.

171 In total, $22 \mathrm{Mg}$ of SMC (between 1 and $5 \mathrm{Mg}$ per HWRC) was collected during two weeks in

172 June 2017 and sorted manually into (Table 1): (i) target waste fraction (plastic film, plastic

173 packaging, glass packaging, metal packaging, polystyrene, paper and board, textile, etc.), (ii)

174 small miscellaneous combustible, and (iii) Other (garden waste, PVC, treated wood, residual

175 household waste). We avoided compacting and sieving in order to distinguish individual

176 material fractions. The samples were transported to the sorting facility in closed containers (30-

$17736 \mathrm{~m}^{3}$ ) to protect waste from weather exposure and minimise any physical changes that may

178 result in inaccurate waste composition data.

179 At the sorting facility, the loaded waste was first sorted according to the type of plastic bags

180 containing the waste, which were (1) black bags, (2) clear bags, (3) other bags and (4) "loose

181 waste" referring to waste disposed without bags. Following, the waste from each bag type was

182 sorted separately.

$183 \quad 2.3$ Data visualisation tools and statistical analysis

$184 \quad 2.3 .1$ Data visualisation tools

185 Various dynamic visualisation techniques were applied to explore and capture insights into 186 waste data prior to statistical analysis. In contrast to classical visualisation plots, geometric mean

187 bar plot (Edjabou et al., 2017a) was used to estimate ratio changes in waste composition. This 188 graph enables to picture systematically changes in percentage of each waste fraction in a group 
189 compared to the average of all the groups, which is considered as a consistent reference. Thus, a

190 negative value indicates that the percentage of a group has decreased compared to the average of

191 all the groups, whereas the contrary is true for a positive value.

192 Ternary diagram (Hamilton, 2017; Pomberger et al., 2017; Thió-Henestrosa and Martín-

193 Fernández, 2005) was employed to simultaneously visualise changes in waste composition for

194 all HWRCs in the period 2010 to 2016. Finally, principal component analysis of waste

195 composition based on a standard covariance biplot for the centered log-ratio (clr-biplot)

196 permitted showing multivariate compositional waste data on a one plot diagram (Aitchison and

197 Greenacre, 2002). These graphs have the advantage of illustrating clearly the variability of

198 individual waste fractions (based on log-ratio transformation) and their relationship.

199 Additionally, they enhance the visibility of the variability of waste fractions and their

200 relationships between several parameters including waste fractional composition, years, HWRC,

201 municipalities and regions (Pawlowsky-Glahn and Egozcue, 2011).

202 For total waste collected and number of visitors, we used the arithmetic mean ratio to 203 quantify and visualise average monthly variation in waste collection during the study period 204 (Cowpertwait and Metcalfe, 2009). This method compares consistently collected waste and 205 number of visitors, regardless of their units.

\section{$206 \quad$ 2.3.2 Statistical analysis methods}

207 The dynamic relationship among waste fractions was addressed applying time series 208 techniques. First, time series decomposition (Ellis, 2016) was used to identify possible seasonal 209 or cyclical patterns and trend in amount of waste collected and its composition. Second, seasonal 210 Kendall test was applied to test for statistical significance of trend and seasonality patterns 211 (Pohlert, 2016) Third, Pettitt's test was applied to ascertain whether the waste collection 
212 significantly changed in the period 2010-2016 (Pohlert, 2016). Finally, autocorrelation function

213 (acf) was used to evaluate the dynamic correlation between waste fractions (Barry, 2016).

214 We also analysed statistical relationship between waste and (i) visitors at the sites, (ii)

215 inhabitants, and (iii) density. This was addressed using the classical Spearman correlation test

216 using symmetric balances (Kynčlová et al., 2017). This latter correlation method addresses the

217 problem of total sum constrain and the interpretation of correlation coefficients is similar to the 218 classical correlation test.

219 For this study, 5\% significance level was used as recommended (European Commission, 220 2004; Nordtest, 1995; US EPA, 2002).

$221 \quad$ 2.3.3 Programming software $R$ packages

222 Data analysis and statistics were carried in R ( $\mathrm{R}$ Core Team, 2017), using tidyverse 223 (Hadley, 2017) and lubridate (Grotemund and Wickham, 2011) for data wrangling; ggplot 224 (Wickham, 2009) for graphics, ggtern for ternary plot (Hamilton, 2017), and Composition (van 225 den Boogaart, 2008) and robComposition (Filzmoser and Hron, 2009; Templ et al., 2011) for 226 compositional analysis techniques.

\section{$227 \quad 3$ Results and discussion}

\section{$228 \quad 3.1$ Waste collected at HWRC}

\section{3. 1.1 Aggregated annual waste collected at HWRC}

230 Total annual waste collected in 36 HWRC is shown in Table 2. Noticeably, the mass of 231 waste was zero from 2010 to 2012 in Bispeengen, because this centre was established in 2013.

232 The results show that the waste collection fluctuated considerably for the period 2010-2016. The 233 highest mass was recorded in 2011 at HWRC of Hvidovre (about 41,000 Mg/year) in Greater 234 Copenhagen, and the lowest was at HWRC of Sejeroe, which is a small island (about 260 $235 \mathrm{Mg} /$ year) in Central Zealand (Table S2). 
The total mass of waste increased sharply in 2011 and declined suddenly in 2012 in most

237 HWRCs (Figure 2). Subsequently, it increased steadily from 2014 to reach almost the same level

238 as in 2011 in Central Zealand, and Silkeborg. Meanwhile, the mass of waste in Djursland 239 showed a downward trend (Figure 2).

240 The waste collection rates computed for each municipality are shown in Table 3. Most 241 waste collection rates decreased steadily from 2012 to 2016 (Table 3 and Figure S1). For 242 example in 2016, waste collection rates in the municipality of Copenhagen were 127 $243 \mathrm{~kg} /$ household/year, $64 \mathrm{~kg} /$ person/year and $93 \mathrm{~kg} /$ visitor/year (Table S3). On the contrary, for the 244 same year, the waste collection rates equalled to $1481 \mathrm{~kg} / \mathrm{household} / \mathrm{year}, 630 \mathrm{~kg} /$ person/year 245 and $68 \mathrm{~kg} /$ visitor/year in Dragoer (Table S3). These results are within the range of estimates in 246 the UK. For instance, 83-292 kg/person/year and $174 \mathrm{~kg} / \mathrm{person} /$ year were reported in 247 Southampton (Woodard et al., 2004) and Altrincham (WRAP, 2012), respectively. Additionally, 248 Norfolk County Council estimated 341- 379 kg/person/year, 753-906 kg/household/year and 249 744-1514 kg/visitor/year (Norfolk County Council, 2015). Consequently, a meaningful 250 comparison among performance of HWRCs should account for the difference between units of 251 waste collection rates.

252 The most striking observation to emerge from Table 3 was the number of visitors per 253 household, which is the ratio between waste collection rates per household and per visitors 254 (Table S2). For illustration, the number of visitors per household in Frederiksberg in 2010 was 2550.43 visitor/household (Table S2 and Figure S1 and S2). The number of visitors per household 256 was lowest in Copenhagen (1.3 visitors/household) and highest in Dragoer (23 257 visitors/household) in the period 2010-2016. However, the average number of person per 258 household were 2.4 and 2.0 in Dragoer and Copenhagen respectively (Table S3). Thus, the key 
difference between these municipalities is the ratio between housing types (single and multi-

260 family houses). In the municipality of Copenhagen about $92 \%$ of houses are multi-family houses

261 where lives about $88 \%$ of the population. Conversely, the municipality of Dragoer accounts $22 \%$

262 of multi-family houses and where $18 \%$ of the population lives (Table S1). This finding indicates

263 that citizen from single-family house areas may tend to visit more often HWRC than those in

264 multi-family house areas. A possible explanation might be that garden waste occurs in single-

265 family houses. Moreover, construction and demolition waste may also arise more often in

266 single-family houses due to activities such as rehabilitation, new construction, etc. However,

267 despite these explanations, care is needed in further interpretation of these results, because

268 municipalities in the Greater Copenhagen are very close and citizens may use any HWRC

269 regardless of the municipalities where they live (Figure 1).

\section{$270 \quad$ 3.1.2 Monthly waste collection}

271 A decomposition plot shown in Figure 3 illustrates the monthly waste collection (Figure

272 3.a), the underlying trend (Figure 3.b), and the seasonal patterns (Figure 3.c). Figure 3.a shows

273 that the total waste collected followed regular cycles, exhibiting clear seasonal patterns displayed

274 in Figure 3.b, which reveals a modest upward trends.

275 The arithmetic mean ratio (in percentage) boxplots for total waste and the number of

276 visitors from all HWRCs is shown Figure 4, displaying the dynamic relationship between the

277 mass of collected waste and the number of visitors at HWRCs. The boxplots are based on data

278 from individual HWRC. Dots show the ratios (mass and the number of visitors) for each month

279 at individual HWRC.

280 Figure 4 illustrates also the seasonal and monhtly variation between the mass of collected 281 waste and the number of visitors enabling to comprehend their dynamic interaction. Here, 
282 negative mean ratios indicate that the monthly value is lower than the average value for the same

283 year, whereas positive mean ratios indicate that the monthly value is higher than the average for

284 the same year (Table S4).

285 In November 2011, extremely high mass was collected from HWRC in Hvidovre,

286 mainly due to cleaning after the storm, which occurred in 2011 and led to flooding in this area.

287 This is illustrated in Figure 4 by a dot that is higher than $100 \%$.

288 The results point out that the total mass and the number of visitors follows the same

289 seasonal patterns. The highest number of sites users and total mass was in April and May,

290 decreased moderately and consistently in summer and autumn months (April to October).

291 Subsequently, the sites users and the mass of waste dropped dramatically to the lowest value in

292 winter from November to March (Figure 4). The results also show that the average mass of waste

293 and number of visitors (red point in each boxplot) were 35-54\% lower than their respective

294 average from December to February. On the other hand, the average mass and number of visitors

295 were 27- 41\% higher than their individual average in May and July.

296 The comparison between number of visitors and total mass could indicate that the

297 average visitor brings more waste when the mean ratio of mass waste is higher than visitors. For

298 example, a visitor at HWRC in May, August, and November, may bring more waste compared to

299 January to April and July (Figure 4).

300 3.1.3 Dynamic patterns and factors affecting the mass of collected waste

301 Table 4 presents the results of the Kendall test. Here, DF is the degree of freedom equalled 302 to 11 (12months minus 1). P-values measure statistical significance for trend ( $\mathrm{z}$ (Trend)) and 303 heterogeneity (Chi-Square (Het)) respectively. The heterogeneity measures the difference 304 between the observed trends. The $95 \%$ confidence intervals refer to the trend values. 
The results shown in Table 4 depict a decreasing trends in Djursland $(-0.39 \mathrm{Mg} /$ year) and

306 Copenhagen $(-0.22 \mathrm{Mg} /$ year) and increasing trends in Silkeborg (1.86 Mg/year) and Central

307 Zealand (1.60 Mg/year), over the period 2010 and 2016 (Figure 3.c). However, the seasonal

308 Kendall test for trend presented in Table 4 (p-values $>0.05$ and two-sided 95\% confidence

309 intervals contain zero value) indicated no statistically significant trend in collected waste. These

310 results may imply that the mass of collected waste in the period 2010 - 2016 did not

311 significantly change. On the other hand, the autocorrelation (ACF) graph (Figure S3) displays a

312 clearly seasonal pattern in mass of collected waste, implying that a time series analysis of waste

313 collection at HWRCs should include a seasonal component.

314 Figure 2 depicts a noticeable increase in total mass of waste collected in these regions in

315 2011. However, the test of change-point detection (Pettitt's test) reveals that it was only in

316 Hvidovre that the collected waste in 2011 (in November) was significantly higher during the

317 study period, for the reason previously explained.

318 The spearman correlation test (Table S6-8) indicates that the number of visitors to HWRCs

319 was highly correlated with discarded waste $(r=0.80-0.96)$. However, there was a weak

320 correlation between the mass of waste and both the number of households $(r=0.32)$ and the

321 number of inhabitants ( $r=0.33$ ) for each municipality. These results suggest that the total waste

322 collected at HWRC may be highly driven by the number of visitors. Moreover, the correlation

323 test (Table S10) also shows that the number of visitors was principally influenced by single-

324 family houses ( $r=0.83$ for number of houses and $r=0.85$ for the number of persons). Owned to

325 these findings, the disparity between waste collection rates $(\mathrm{kg} /$ person/year and $\mathrm{kg} / \mathrm{visitor} / \mathrm{year})$

326 shown in Table 3 may be explained by the ratio between single-family and multi-family

327 households. For example, the estimated waste collection rates per visitor were higher than per 
328 inhabitant in Copenhagen and Frederiksberg (2013-2016), where about 92 - $97 \%$ of household 329 are multi-family houses (Table S1).

$330 \quad 3.2$ Waste composition based on main fractions

3313.21 Aggregated yearly waste composition based on main fractions

332 The yearly composition of main waste categories is shown in Table 2. The waste collected

333 at HWRC mainly consisted of construction and demolition (C\&D) waste in Greater Copenhagen

334 (24-30\%), and garden waste (24-32\%) in other regions (Central Zealand, Djursland and

335 Silkeborg). A plausible explanation is that Greater Copenhagen is the capital city. It consists 336 mainly of multi-family houses (Table S1), generating less garden waste. Additionally, there may

337 be more building activities in the capital city generating construction and demolition waste.

338 The target waste fractions represented was one of the predominant waste fractions 339 occurring in these regions. Remarkably, the percentage of target waste nearly doubled (from 13 340 to 22\%) in Greater Copenhagen from 2010 to 2011 (Table 2). This is due to establishment of a 341 new source segregation fraction, namely wood (untreated wood), to substitute bulky waste, 342 which were removed from HWRCs.

343 Overall, small miscellaneous combustible and bulky waste accounted for more than $10 \%$ of 344 total waste at HWRCs (Table 2). Moreover, the percentage distribution between main waste 345 fractions vary considerably between regions. This is illustrated in Figure 5 (Clr-biplot) 346 according to two distinct groups: (1) Djursland and (2) other areas (Central Zealand, Greater 347 Copenhagen, and Silkeborg). Figure 5 indicates also that waste composition at HWRCs from 348 where small miscellaneous combustible waste was sampled and characterised (Grenaa, 349 Hvidovre, Jyllinge, Tandskov, Tietgensvej, Vermlandsgade and Viby) exhibited similar patterns 350 as other HWRCs in their respective group. The difference between these groups is mainly due to 
351 "other waste" representing the second largest categories in Djursland (18-23\%). The underlying 352 reason is that Djursland is located in rural area with large landfill capacity. As a result, this area

353 receives considerable amount of landfill waste from neighbouring municipalities, which is 354 accounted as waste from HWRC. On the contrary, "other waste" accounted less than $12 \%$ in 355 other regions. Moreover, Figure 5 depicts that while wood was predominant main fraction in

356 Greater Copenhagen, fibres and other (paper, board and textile), soil and construction and 357 demolition waste were also prevalent in this region. We also notice that HWRCs in Greater 358 Copenhagen can be further subdivided into two groups: (1) HWRCs located in areas with 359 prevalence of single-family houses with garden (Dragoer) and (2) those located in areas with 360 prevalence of multi-family houses such as Copenhagen, Frederiksberg and Taarnby. The 361 difference between these two sub-groups in Greater Copenhagen was that target waste fraction 362 (mainly wood) was predominant in the HWRCs located in the neighbourhood of multi-family

363 houses, whereas soil and garden waste constituted the principal categories in HWRCs located 364 close to single family houses with garden namely Taarnby (Table S11 and S12 for detail waste 365 composition).

\section{$366 \quad 3.2 .2$ Monthly waste composition based on main fractions}

367 Multivariate analysis show that the composition of most waste fractions exhibited a 368 significant seasonal variation patterns (Table S9). Additionally, a generalised univariate analysis 369 (Table S10-11) indicate a significantly seasonal patterns for most main categories and HWRCs 370 excluding Christiania (Copenhagen), Sejeroe (Central Zealand), and Roende (Djursland). These

371 later HWRCs recorded relatively small mass (Table 1), which fluctuates independently of 372 monthly variation.

373 While the relationship between the collection of garden waste and seasonal variation is 
374 quite well documented (Boldrin and Christensen, 2010), these results suggest that the collection

375 of most main fractions at HWRCs may be also significantly associated with seasonal variations.

\section{$376 \quad 3.3$ Variation of waste treatments forms}

377 A comparative composition of waste fractions based on treatment forms is illustrated in

378 Figure 6. The percentage of waste for other treatment forms was lowest (9-14\%), whereas

379 recycling $(65-75 \%)$ was the largest waste treatment forms followed by incineration (14-19\%).

380 The geometric bar plot (Figure 7) reveals an overall relative increase in the percentage of

381 recycling and a relative decrease of incineration. Moreover, there was a relative small change in

382 the composition of waste treatment forms in Djursland compared to other areas. Although the

383 percentage of other treatment form was the smallest and almost constant in Figure 6, it

384 fluctuated considerably during this period as shown in Figure 7. For example, the percentage of

385 other treatment forms was $30 \%$ higher than the average in 2010 , and $24 \%$ smaller than the

386 average in 2016. Figure 7 shows that the percentage of recycling decreased from 22 to $4 \%$ in

387 Greater Copenhagen in 2016. This may be explained by the new regulation banning the

388 recycling of construction and demolition waste contaminated or suspected to be contaminated

389 with PCB (Christensen et al., 2016). Figure 7 also highlights a drastic relative change in

390 percentage of incinerated waste in Greater Copenhagen (74 to 4 higher than average) and "other

391 treatment" ( 85 higher to 12 lower than average) in 2011. This sudden decrease was mainly due

392 to the establishment of wood waste as a separate waste fraction in HWRCs.

393 To compare the performance of individual HWRCs, we used ternary plot presented in

394 Figure 8, where three groups of HWRCs are distinguished: (1) Christiania (from Greater

395 Copenhagen area) and Sejeroe (from Central Zealand) where the lowest mass of waste was

396 recorded; (2) all HWRCs in Djursland, and (3) other HWRCs from Central Zealand, Greater 
397 Copenhagen and Silkeborg. The difference between the second and the third group was the 398 percentage of waste treated by means of other treatment forms, which was $15-25 \%$ in Djursland 399 and less than $20 \%$ in the third group. Additionally, a moderate decrease in the percentage of 400 incinerated waste was observed in Djursland (15 to 12\%). On the other hand, there was a 401 considerable decrease in incinerated waste in the third group (40 to 13\%). Figure 8 indicates also 402 that the performance of monitored HWRCs was comparable to other HWRCs in the 403 distinguished groups.

\section{$404 \quad 3.4$ Effectiveness of black plastic policy}

405 The characterisation of $22 \mathrm{Mg}$ of small miscellaneous combustible (SMC) from seven 406 HWRC reveals that 50\% (41-57\%) of SMC was disposed of in HWRC without using bag. On 407 the other hand, $23 \%(11-36 \%)$ and 19\% (12-25\%) of SMC was disposed of using clear, and 408 mixed plastic bags, respectively. Moreover, 7\% (3- 9\%) waste was brought in black bags, 409 although it was forbidden. Seemingly, the percentage of SMC brought in black bag was 410 relatively lower compared to other bags (Figure 9). The results showed that black bags and other

411 bags contained principally target waste fractions. This finding suggests that misplaced target 412 waste fractions could be considerably reduced if users bring their waste mainly using clear 413 plastic bags.

414 The data revealed also that overall, only $32 \%$ (19-44\%) of waste was SMC, suggesting that 415 about $68 \%(56-81 \%)$ of waste was misplaced materials, consisting of $49 \%(41-60 \%)$ target 416 waste fractions and 18\% (13-35\%) hazardous and other waste. These results indicate that 417 considerable efforts and initiative are needed to reduce significantly the percentage of misplaced 418 waste in containers for SMC. They may also support the "believe" that citizens tend to dispose 419 of their waste in the container for small miscellaneous combustible when they are in doubt. 
420 Importantly, some of the clear plastic bags contained only target waste fractions. This may

421 suggest that although some citizens may correctly sort their waste at home, they unfortunately

422 dispose their sorted waste in the container of SMC. Therefore, an adequate assistance to users,

423 such as the "meet-and-greet staff" strategy (WRAP, 2012), could provide help to users in

424 disposing their waste in the appropriate waste containers.

425 Figure 10 show the potential recycling rates achievable if no target waste and hazardous 426 waste are disposed in container for SMC. For most of the HWRCs, there would be an obvious

427 increase in recycling rates, as well as other waste treatment forms. Noticeably, the percentage of 428 incinerated waste would decrease considerably from 32 to $15 \%$ in Viby (red arrow in Figure 10 429 moving from 2016 to the estimated potential recycling rates) and Jylling, whilst only a $3 \%$ 430 increase in the recycling rate would be observed in Hvidovre. These results highlight that the 431 effort and benefit to reduce misplacement of target recycling waste vary according to the 432 HWRC, because of the difference in the percentage of SMC. For example, in 2016, the small 433 miscellaneous combustible waste amounted to 5-7\% in Greater Copenhagen, Djusrland and 434 Silkeborg, and $11 \%$ in Central Zealand.

\section{$435 \quad 3.5$ Implications and perspectives}

436 The waste analysis shows that the waste collection at HWRC was mainly driven by the 437 number of visitors, which was significantly affected by the number of single-family houses. The 438 large disparity in waste collection rates between municipalities may cause serious inconsistency 439 in comparing the performance of HWRCs.

440 The findings of this study urge waste practitioners and researchers to explore new dynamic 441 visualisation tools to enclose features contained in waste data and consistently compare waste 442 composition data. Various data visualisation tools were used to fully understand and capture 
443 patterns in waste collection and composition data. Stacked bar plot (Figure 6) visualises the 444 distribution between waste treatment forms in a quite intuitive manner. However, changes in 445 "other treatment" are barely noticeable and direct comparison between areas was not striking. 446 Fortunately, these limitations are transcended by geometric bar plot (Figure 7), which quantify 447 relative changes regardless of the size of the fraction (percentage). Furthermore, the trend in 448 waste treatment forms were perceptible (and the comparison between areas were directly 449 feasible in Figure 7. Markedly, recycling has increased whereas incineration and "other 450 treatment" have decreased. Despite these advantages, presenting a geometric bar plot of 36 451 HWRCs in one page may be overwhelming and technically challenging. Nevertheless, this is an 452 area in which ternary plot excels. Figure 8 allows presenting the treatment forms of $36 \mathrm{HWRCs,}$ 453 from 2010 to 2016 . This visualisation enables to monitor the past, the current and to model the 454 future paths as shown in Figure 11. Additionally, ternary plot enables a direct comparison 455 between many parameters by grouping the HWRC having the same patterns. However, ternary 456 plot can be used only for illustrating three parameters at a time (here waste treatment forms). An 457 alternative could be clr-biplot, which take into consideration the inherent nature of 458 compositional datasets.

459 The results of seasonal analysis indicates the total waste collection as well as main waste 460 fraction follow seasonal patterns. Importantly, seasonal variation significantly affected the 461 number of visitors, which was strongly correlated with the waste collection. These results imply 462 that a public awareness campaigns should be carried out in the period April-September in order 463 to target a broader and larger audience.

464 Characterisation of SMC provided substantial information about misplacement rates. This 465 may also suggest that characterisation of all fractions sorted at HWRC is required in in mapping 
466 impurity and misplacement rates. This information contributes to optimise waste collection and

467 increase the purity of recyclable materials and thereby conduct a reliable material flow analysis 468 as well as environmental impact assessment of a HWRC.

\section{Conclusions}

470 The purpose of this study was to analyse historical waste compositional data to enable an

471 optimal planning of household waste recycling centres. This study identified that the mass of

472 waste collected at recycling centres was significantly correlated with the number of visitors.

473 Moreover, the number of visitors and waste collection followed a seasonal pattern. The seasonal

474 Kendall test for trend revealed no significant trend in waste collection suggesting that it did not

475 significantly change during the period 2010-2016. The results also showed a large discrepancy in 476 waste collection rates (kg/visitors/year, $\mathrm{kg} /$ inhabitant/year, and kg/household/year), which could

477 be explained by the ratio between number of visitors and single-family houses. However, a

478 further historical data analysis of all household waste streams may provide a comprehensive 479 understanding of the discrepancy between waste collection rates. This may help identifying the 480 factors influencing waste collection and its composition.

481 We also observed an increase in recyclable rates and a decrease in the percentage of

482 incinerated waste for the 36 HWRCs involved in the study period 2010-2016. This demonstrates

483 the importance of ternary plot for comparing directly multiple parameters.

484 The characterisation of small miscellaneous combustible waste suggested that about $7 \%$

485 of small miscellaneous combustible was brought in black plastic bags. Overall, about $68 \%$ of

486 small miscellaneous combustible was misplaced materials consisting of recyclable materials

487 (49\%) and hazardous waste and other (18\%). These results suggest that a consistent material 
488 flow analysis of waste from recycling centres may require a characterising of source-segregated 489 waste fractions in order to estimate misplacement rates.

\section{Acknowledgments}

491 The authors acknowledge the Danish Environmental Protection Agency (EPA), the waste 492 management companies ARC (Amager resource Centre), ARGO (Affald Resourcer, Genbrug

493 Overskud (en: waste, resource,recycling, benefit)), Silkeborg forsyning, and RenoDjurs for 494 providing waste data. Additionally, we will like to express our gratitude to Claus Petersen, Hardy

495 Mikkelsen, Karsten Søndergaard, Ejvind Mortensen and Linda Rebien for the diverse 496 contribution to the manuscript. 
Aitchison, J., 1994. A Concise Guide to Compositional Data Analysis. Lecture NotesMonograph Series 24, 73-81.

Aitchison, J., Greenacre, M., 2002. Biplots of Compositional Data. Applied Statistics 51, 375392. doi:10.1111/1467-9876.00275

RVF, 2018. Afvallshantering, hushållsavfall/Municipal solid waste management. RVF Report 2018, (RVF)/The Swedish Association of Waste Management, Malmö, Sweden (in Swedish).

Avfall Norge, 2019. Husholdningsrenovasjon 2018. Resultater fra enchmarking

Barry, D., 2016. Business Intelligence with R From Acquiring Data to Pattern Exploration.

Bigum, M., Petersen, C., Christensen, T.H., Scheutz, C., 2013. WEEE and portable batteries in residual household waste: Quantification and characterisation of misplaced waste. Waste management (New York, N.Y.) 33, 2372-80. doi:10.1016/j.wasman.2013.05.019

Boldrin, A., Christensen, T.H., 2010. Seasonal generation and composition of garden waste in Aarhus (Denmark). Waste Management 30, 551-557. doi:10.1016/j.wasman.2009.11.031

Buccianti, A., Pawlowsky-Glahn, V., 2011. Compositional Data Analysis, Compositional Data Analysis: Theory and Applications. John Wiley \& Sons, Ltd, Chichester, UK. doi:10.1002/9781119976462

Christensen, F., Warming, M., Kirkeby, J.T., Maag, J., Steinhausen, J., Hansen, H.J., 2016. Kemiske stoffer i forbrugerprodukter, der kan hindre genanvendelse - Udvikling af metode Kortlægning af kemiske stoffer i forbruger- produkter. Danish Environmental Protection Agency, Copenhagen, Denmark.

Cowpertwait, P.S.P., Metcalfe, A. V., 2009. Introductory Time Series with R, Media. New York, New York, USA. doi:10.1007/978-0-387-88698-5

Danish EPA, 2018. Portal for waste recycling [WWW Document]. URL http://genanvend.mst.dk/om-genanvendmstdk/ (accessed 4.5.18).

Danish Government, 2013. Denmark without waste: recycle more -incinerate less. Danish Ministry of the Environment, Copenhagen, Denmark. doi:978-87-03026-59-5

Edjabou, M.E., Boldrin, A., Astrup, T.F., 2018. Compositional analysis of seasonal variation in Danish residual household waste. Resources, Conservation and Recycling 130. doi:10.1016/j.resconrec.2017.11.013

Edjabou, M.E., Boldrin, A., Astrup, T.F., 2017a. Compositional analysis of seasonal variation in Danish residual household waste. Resources, Conservation and Recycling. doi:10.1016/j.resconrec.2017.11.013

Edjabou, M.E., Martín-Fernández, J.A., Scheutz, C., Astrup, T.F., 2017b. Statistical analysis of solid waste composition data: Arithmetic mean, standard deviation and correlation coefficients. Waste Management 69, 13-23. doi:10.1016/j.wasman.2017.08.036

Ellis, P., 2018. ggseas: "stats" for Seasonal Adjustment on the Fly with "ggplot2." R package version 0.5.4. Available at: https://CRAN.R-project.org/package=ggseas

European Commission, 2016. Council conclusions on the EU action plan for the circular economy. SWD/2015/0259 final - 2015/0275 (COD). Available at: http://eur?lex.europa.eu/legal?content/EN/TXT/?uri=CELEX:52015SC0259

Filzmoser, P., Hron, K., 2009. Correlation analysis for compositional data. Mathematical Geosciences 41, 905-919. doi:10.1007/s11004-008-9196-y

Filzmoser, P., Hron, K., 2008. Correlation Analysis for Compositional Data. Mathematical 
Geosciences 41, 905-919. doi:10.1007/s11004-008-9196-y

Grotemund, G., Wickham, H., 2011. Dates and Times Made Easy with lubridate. Journal of Statistical Software (40),3,p1-25. Available at: http://www.jstatsoft.org/v40/i03/

Hadley, W., 2018. tidyverse: Easily Install and Load the 'Tidyverse'. R package version 1.2.1. https://CRAN.R-project.org/package=tidyverse.

Hamilton, N., 2018. ggtern: An Extension to "ggplot2", for the Creation of Ternary Diagrams. R package version 3.0.0. Available at: https:/CRAN.R-project.org/package=ggtern

Katsamaki, A., Willems, S., Diamadopoulos, E., 1998. Time Series Analysis of Municipal Solid Waste Generation Rates. Journal of Environmental Engineering 124, 178-183. doi:10.1061/(ASCE)0733-9372(1998)124:2(178)

Krook, J., Eklund, M., 2010. Developing a monitoring method facilitating continual improvements in the sorting of waste at recycling centres. Waste Management 30, 32-40. doi:10.1016/j.wasman.2009.08.009

Kynčlová, P., Filzmoser, P., Hron, K., 2015. Modeling compositional time series with vector autoregressive models. Journal of Forecasting 34, 303-314. doi:10.1002/for.2336

Kynčlová, P., Hron, K., Filzmoser, P., 2017. Correlation Between Compositional Parts Based on Symmetric Balances. Mathematical Geosciences. doi:10.1007/s11004-016-9669-3

Martinez-Sanchez, V., Hulgaard, T., Hindsgaul, C., Riber, C., Kamuk, B., Astrup, T.F., 2016. Estimation of marginal costs at existing waste treatment facilities. Waste Management 50, 364-375. doi:10.1016/j.wasman.2016.02.032

Navarro-Esbrí, J., Diamadopoulos, E., Ginestar, D., 2002. Time series analysis and forecasting techniques for municipal solid waste management. Resources, Conservation and Recycling 35, 201-214. doi:10.1016/S0921-3449(02)00002-2

Nilsson, P., Christensen, T.H., 2010. Waste Collection: Systems and Organization, in: Christensen, T.H. (Ed.), Solid Waste Technology \& Management, Volume $1 \& 2$. Chichester, UK. doi:10.1002/9780470666883.ch21

Norfolk County Council, 2015. Provision of Household Waste Recycling Centre Services Appendix M - Recycling Centre Infrastructure Strategy 2015. Appendix M- Recycling Centre Strategy.

Nordtest. 1995. "Municipal Solid Waste: Sampling and Characterisation.” NT ENVIR 001. Nordtest Method. Espoo, Finland. http://www.nordtest.info/images/documents/ntmethods/environment/NT envir 001_Solid waste, municipal_Sampling and characterisation_Nordtest Method.pdf.

Ordoñez, I., Harder, R., Nikitas, A., Rahe, U., 2014. Waste sorting in apartments: integrating the perspective of the user. J. Clean. Prod. doi:10.1016/j.jclepro.2014.09.100

Pawlowsky-Glahn, V., Egozcue, J., 2011. Exploring Compositional Data with the CoDaDendrogram. Austrian Journal of Statistics 40, 103-113.

Pawlowsky-Glahn, V., Egozcue, J., Tolosana-Delgado, R., 2015. Modeling and Analysis of Compositional Data. John Wiley \& Sons.

Pohlert, T., 2016. Non-Parametric Trend Tests and Change-Point Detection. R package 26. doi:10.13140/RG.2.1.2633.4243

Pomberger, R., Sarc, R., Lorber, K.E., 2017. Dynamic visualisation of municipal waste management performance in the EU using Ternary Diagram method. Waste Management 1-14. doi:10.1016/j.wasman.2017.01.018

R Core Team, 2018. R: A Language and Environment for Statistical Computing. R Foundation for Statistical Computing. Vienna, Austria. Available at: https://www.R-project.org/ 
Statistics Denmark, 2017. BOL103: Dwellings by county, type of resident, type of dwelling, number of rooms, size of dwelling in square metre and household size [WWW Document]. URL http://www.statistikbanken.dk/BOL103

Templ, M., Hron, K., Filzmoser, P., 2011. robCompositions: an R-package for robust statistical analysis of compositional data, in: Pawlowsky-Glahn, V., Buccianti, A. (Eds.), Compositional Data Analysis. Theory and Applications. John Wiley \& Sons, Chichester (UK), pp. 341-355.

Thió-Henestrosa, S., Martín-Fernández, J.A., 2005. Dealing with compositional data: The freeware CoDaPack. Mathematical Geology 37, 773-793. doi:10.1007/s11004-005-7379-3

Toft, R., Nissen, E.L., Sørensen, A., 2017. Affaldsstatistik (Waste statistitics) 2015. Ministery of environment and food-Danish Environmental Agency (EPA).

US EPA, OSWER, O. of R.C. and R., 2017. Resource Conservation | Wastes | US EPA.

US EPA, 2002. RCRA Waste Sampling Draft Technical Guidance Planning, Implementation, and Assessment (No. EPA530-D-02-002), Solid Waste and Emergency Response (5305W). <http://www3.epa.gov/epawaste/hazard/ testmethods/sw846/pdfs/rwsdtg_a.pdf>. van den Boogaart, K.G., 2008. "compositions": A unified R package to analyze compositional data. COMPUTERS AND GEOSCIENCES 34, 320-338.

Wickham, H., 2009. ggplot2: Elegant Graphics for Data Analysis. Springer-Verlag New York. Woodard, R., Bench, M., Harder, M.K., Stantzos, N., 2004. The optimisation of household waste recycling centres for increased recycling - a case study in Sussex, UK. Resources, Conservation and Recycling 43, 75-93. doi: 10.1016/j.resconrec.2004.05.002

WRAP, 2012. Household Waste Recycling Centre (HWRC) Guide. p127. www.wrap.org.uk 


\section{Temporal and geographical patterns of solid waste collected at recycling}

\section{centres}

Maklawe Essonanawe Edjabou*, Giorgia Faraca, Alessio Boldrin, and Thomas Fruergaard Astrup

Department of Environmental Engineering, Technical University of Denmark, 2800 Kgs. Lyngby, Denmark

*) Corresponding author: vine@env.dtu.dk;

Phone number: +4545251498 


\section{List of Tables}

Table 1: List of waste fraction disposed of at HWRC grouped into Waste treatment forms, Main waste fraction, and Waste target fractions

\begin{tabular}{|c|c|c|}
\hline Waste Treatments & Main fractions & List of components \\
\hline \multirow[b]{2}{*}{ Incineration } & Bulky & (1) Furniture, (2) sludge and Other bulky waste \\
\hline & $\begin{array}{l}\text { Small miscellaneous combustibles } \\
\text { (SMC) }\end{array}$ & $\begin{array}{l}\text { (3)Residual household waste } \\
\text { (4) Miscellaneous combustible }\end{array}$ \\
\hline \multirow{8}{*}{ Recycling } & $\begin{array}{l}\text { Construction and demolition waste } \\
\text { (C\&D) }\end{array}$ & $\begin{array}{l}\text { (5) Asphalt residues, (6) bricks, (7) mixed bricks, (8) blocs and tiles, (9) } \\
\text { concrete, (10) Gypsum waste, (11) sanitary ware, (12) tiles, (13) waste } \\
\text { exchange }\end{array}$ \\
\hline & Garden waste & (14) Garden waste \\
\hline & Soil & (15) Soil \\
\hline & Fibres and other $^{\mathrm{a}}$ & (16) Paper and board, (17) Textiles \\
\hline & Glass $^{\mathrm{a}}$ & (18) Glass packaging, (19) window glass \\
\hline & Metal $^{\mathrm{a}}$ & $\begin{array}{l}\text { (20) Cables, (21) ferrous metal, (22) metal containers and cans, (23) non- } \\
\text { ferrous metal }\end{array}$ \\
\hline & Plastic $^{\mathrm{a}}$ & $\begin{array}{l}\text { (24) hard plastics, }(25) \text { plastic composite, }(26) \text { plastic film, (27) plastic } \\
\text { garden furniture, }(28) \text { polystyrene }\end{array}$ \\
\hline & Wood $^{\text {a }}$ & (29) untreated wood \\
\hline Other $^{\mathrm{b}}$ & Other waste & $\begin{array}{l}\text { (30) Anti-freeze, (31) asbestos, (32) waste, (33) batteries, (34) big bags, } \\
\text { (35) CDs and DVDs, (36) chemical fertilize, (37) clinical waste, (38) } \\
\text { cooling and heating white goods, (39) fire extinguisher, } \\
\text { (40) fireworks, (41) garden herbicides and pesticides, (42) gas bottles, } \\
\text { (43) landfilled waste, (44) large household Appliances, (45) lighting } \\
\text { equipment, mattresses, } \\
\text { (46) mineral oil, (47) mixed waste, (48) other wood, (49) paints, (50) } \\
\text { polyvinylchloride (PVC), (51) printer cartridges, (52) small household } \\
\text { appliances, (53) solvent-based waste, } \\
\text { (54) spray can, (55) thermal insulation materials, (56) treated wood, (57) } \\
\text { tyres, untreated wood, (58) waste accumulators, (59) windows glass with } \\
\text { PCB }\end{array}$ \\
\hline
\end{tabular}

${ }^{a}$ Target waste fractions consisted of Fibres and textiles, Glass, Metal, Plastic and wood; ${ }^{b}$ Landfill, temporarily storage and special treatments 
Table 2: Waste composition [\% wet mass basis] at HWRC based on main waste categories

\begin{tabular}{|c|c|c|c|c|c|c|c|c|}
\hline \multirow[b]{2}{*}{ Areas } & \multirow[b]{2}{*}{ MWC $^{\mathrm{a}}$} & \multicolumn{7}{|c|}{ Waste composition [\%] } \\
\hline & & 2010 & 2011 & 2012 & 2013 & 2014 & 2015 & 2016 \\
\hline \multirow{13}{*}{ Greater Copenhagen } & Fibres $^{\mathrm{c}}$ & 2.80 & 2.60 & 2.60 & 2.70 & 2.60 & 2.60 & 2.90 \\
\hline & Plastic & 0.10 & 0.10 & 0.30 & 0.70 & 0.90 & 0.90 & 1.00 \\
\hline & Metal & 4.70 & 4.40 & 4.20 & 4.10 & 3.80 & 3.90 & 4.20 \\
\hline & Wood & 4.00 & 13.50 & 13.90 & 14.70 & 14.80 & 15.70 & 15.90 \\
\hline & Glass & 1.40 & 1.40 & 1.10 & 1.10 & 1.00 & 1.10 & 1.00 \\
\hline & $T W F^{b}$ & 13.00 & 22.00 & 22.10 & 23.30 & 23.10 & 24.20 & 25.00 \\
\hline & Garden & 12.30 & 12.90 & 11.90 & 12.60 & 13.00 & 12.60 & 12.90 \\
\hline & Soil & 14.10 & 14.00 & 15.40 & 14.10 & 14.50 & 14.50 & 13.90 \\
\hline & C\&D & 24.40 & 31.40 & 32.50 & 32.10 & 32.80 & 32.60 & 30.80 \\
\hline & SMC & 12.40 & 11.00 & 9.60 & 9.40 & 8.70 & 8.10 & 7.80 \\
\hline & Bulky & 8.00 & 1.20 & 0.40 & 1.30 & 1.40 & 1.30 & 1.40 \\
\hline & OW & 15.80 & 7.50 & 8.00 & 7.10 & 6.40 & 6.70 & 8.20 \\
\hline & Total $^{\mathrm{d}}$ & 100.00 & 100.00 & 100.00 & 100.00 & 100.00 & 100.00 & 100.00 \\
\hline \multirow{6}{*}{$\mathrm{Mg} /$ year wet mass ${ }^{\mathrm{f}}$} & Total & 106156 & 123789 & 93360 & 90652 & 99548 & 104371 & 104521 \\
\hline & Fibres $^{\mathfrak{c}}$ & 2.30 & 2.00 & 2.10 & 2.10 & 1.90 & 1.90 & 1.80 \\
\hline & Plastic & 0.00 & 0.00 & 0.00 & 0.10 & 0.10 & 0.10 & 0.10 \\
\hline & Metal & 4.10 & 4.30 & 4.30 & 3.90 & 4.30 & 4.30 & 4.70 \\
\hline & Wood & 0.10 & 0.00 & 0.00 & 0.00 & 0.00 & 2.30 & 6.60 \\
\hline & Glass & 2.20 & 2.30 & 2.70 & 2.30 & 2.30 & 2.40 & 2.00 \\
\hline \multirow[t]{8}{*}{ Central Zealand } & $T W F^{b}$ & 8.70 & 8.60 & 9.10 & 8.40 & 8.60 & 11.00 & 15.20 \\
\hline & Garden & 26.00 & 24.20 & 25.70 & 25.30 & 25.80 & 25.50 & 25.90 \\
\hline & Soil & 8.60 & 7.80 & 7.90 & 7.30 & 8.20 & 8.10 & 8.10 \\
\hline & C\&D & 24.00 & 23.80 & 24.00 & 23.90 & 25.10 & 25.70 & 25.40 \\
\hline & SMC & 12.00 & 18.00 & 12.30 & 12.60 & 13.60 & 14.20 & 11.10 \\
\hline & Bulky & 11.70 & 8.40 & 12.90 & 13.10 & 11.90 & 9.50 & 7.90 \\
\hline & OW & 8.90 & 9.20 & 8.00 & 9.50 & 6.80 & 6.00 & 6.30 \\
\hline & Total $^{\mathrm{d}}$ & 100.00 & 100.00 & 100.00 & 100.00 & 100.00 & 100.00 & 100.00 \\
\hline \multirow[t]{6}{*}{ Mg/year wet mass ${ }^{\mathrm{f}}$} & Total & 118773 & 147437 & 126747 & 127689 & 132580 & 138100 & 140811 \\
\hline & Fibres $^{\mathrm{c}}$ & 1.90 & 1.90 & 1.80 & 1.80 & 1.70 & 1.80 & 2.30 \\
\hline & Plastic & 0.70 & 0.90 & 0.90 & 1.00 & 0.90 & 1.00 & 1.00 \\
\hline & Metal & 4.30 & 4.70 & 4.40 & 4.50 & 4.40 & 5.00 & 5.00 \\
\hline & Wood & 7.00 & 7.10 & 7.00 & 7.20 & 7.00 & 7.90 & 8.70 \\
\hline & Glass & 0.80 & 1.60 & 1.80 & 1.80 & 1.70 & 2.00 & 2.20 \\
\hline \multirow[t]{8}{*}{ Djursland } & $T W F^{b}$ & 14.70 & 16.20 & 15.90 & 16.30 & 15.70 & 17.70 & 19.20 \\
\hline & Garden & 28.50 & 29.60 & 33.10 & 34.40 & 37.50 & 33.30 & 32.30 \\
\hline & Soil & 9.70 & 6.90 & 7.00 & 6.00 & 6.20 & 6.40 & 6.50 \\
\hline & C\&D & 14.80 & 16.00 & 15.20 & 14.60 & 14.00 & 14.50 & 13.70 \\
\hline & SMC & 6.00 & 6.10 & 5.80 & 5.80 & 5.40 & 5.50 & 5.50 \\
\hline & Bulky & 3.50 & 3.30 & 3.30 & 3.30 & 3.00 & 3.00 & 3.20 \\
\hline & OW & 22.80 & 22.00 & 19.60 & 19.70 & 18.20 & 19.70 & 19.60 \\
\hline & Total $^{\mathrm{d}}$ & 100.00 & 100.00 & 100.00 & 100.00 & 100.00 & 100.00 & 100.00 \\
\hline \multirow[t]{6}{*}{$\mathrm{Mg} /$ year wet mass $^{\mathrm{f}}$} & Total & 40320 & 42861 & 40841 & 39251 & 42921 & 40358 & 37254 \\
\hline & Fibres $^{\mathrm{c}}$ & 3.70 & 2.80 & 3.40 & 3.90 & 4.10 & 3.60 & 3.80 \\
\hline & Plastic & 0.10 & 0.20 & 1.00 & 1.20 & 1.20 & 1.30 & 1.20 \\
\hline & Metal & 4.20 & 4.10 & 3.90 & 3.90 & 3.70 & 3.90 & 4.00 \\
\hline & Wood & 6.00 & 8.30 & 9.70 & 10.80 & 11.30 & 11.10 & 11.10 \\
\hline & Glass & 1.70 & 2.00 & 2.50 & 2.50 & 2.50 & 2.40 & 2.40 \\
\hline \multirow[t]{8}{*}{ Silkeborg } & $T W F^{b}$ & 15.70 & 17.40 & 20.50 & 22.30 & 22.80 & 22.30 & 22.50 \\
\hline & Garden & 26.00 & 27.00 & 28.30 & 26.50 & 27.70 & 27.60 & 27.00 \\
\hline & Soil & 10.30 & 10.50 & 10.80 & 11.30 & 11.70 & 11.60 & 11.10 \\
\hline & C\&D & 20.50 & 22.30 & 21.70 & 21.50 & 20.90 & 21.80 & 22.20 \\
\hline & SMC & 10.50 & 10.10 & 8.10 & 8.00 & 6.90 & 6.70 & 6.00 \\
\hline & Bulky & 6.70 & 5.00 & 3.40 & 3.00 & 2.80 & 3.10 & 3.40 \\
\hline & OW & 10.30 & 7.80 & 7.20 & 7.40 & 7.20 & 6.90 & 7.00 \\
\hline & Total $^{\mathrm{d}}$ & 100.00 & 100.00 & 100.00 & 100.00 & 100.00 & 100.00 & 100.00 \\
\hline $\mathrm{Mg} /$ year wet mass ${ }^{\mathrm{f}}$ & Total & 39306 & 41181 & 38324 & 37554 & 39523 & 40938 & 41389 \\
\hline
\end{tabular}

${ }^{a}$ Main waste categories; ${ }^{b}$ target waste fractions; ${ }^{c}$ Fibers includes paper, board and textiles. ${ }^{\mathrm{d}}$ Total is rounded to 100.00. Due to significant digit,

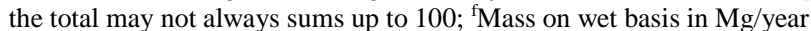


Table 3: Aggregated yearly waste generation rates (wet mass), expressed in $\mathrm{kg} / \mathrm{household} / \mathrm{year}$ for $\mathrm{WCR}_{\text {house, }}$ $\mathrm{kg} /$ inhabitant/year for $\mathrm{WCR}_{\text {inhabitant, }}$ and $\mathrm{kg} /$ visitors/year for $\mathrm{WCR}_{\text {visitor }}$

\begin{tabular}{|c|c|c|c|c|c|c|c|c|}
\hline \multirow{2}{*}{ Municipalities } & \multirow{2}{*}{ Parameter } & \multicolumn{7}{|c|}{ Years } \\
\hline & & 2010 & 2011 & 2012 & 2013 & 2014 & 2015 & 2016 \\
\hline \multirow{3}{*}{ Greater Copenhagen } & $\mathrm{WCR}_{\text {house }}$ & 326 & 377 & 244 & 235 & 257 & 268 & 266 \\
\hline & $\mathrm{WCR}_{\text {inhabitant }}$ & 171 & 195 & 125 & 120 & 129 & 133 & 132 \\
\hline & $\mathrm{WCR}_{\text {visitor }}$ & 118 & 125 & 117 & 112 & 98 & 97 & 98 \\
\hline \multirow{3}{*}{ Central Zealand } & $\mathrm{WCR}_{\text {house }}$ & 1143 & 1407 & 1205 & 1206 & 1243 & 1283 & 1299 \\
\hline & $\mathrm{WCR}_{\text {inhabitant }}$ & 507 & 627 & 538 & 541 & 558 & 576 & 583 \\
\hline & $\mathrm{WCR}_{\text {visitor }}$ & - & - & 172 & 131 & 130 & 121 & 127 \\
\hline \multirow{3}{*}{ Djursland } & $\mathrm{WCR}_{\text {house }}$ & 1128 & 1193 & 1134 & 1080 & 1172 & 1095 & 1002 \\
\hline & $\mathrm{WCR}_{\text {inhabitant }}$ & 512 & 543 & 518 & 496 & 543 & 511 & 469 \\
\hline & $\mathrm{WCR}_{\text {visitor }}$ & 103 & 100 & 99 & 95 & 100 & 90 & 88 \\
\hline \multirow[b]{2}{*}{ Silkeborg } & $\mathrm{WCR}_{\text {house }}$ & 1020 & 1066 & 988 & 963 & 1008 & 1037 & 1035 \\
\hline & $\mathrm{WCR}_{\text {inhabitant }}$ & 448 & 467 & 433 & 424 & 444 & 458 & 459 \\
\hline
\end{tabular}

${ }^{\mathrm{a}} \mathrm{kg} / \mathrm{visitor} / \mathrm{year}$; ${ }^{\mathrm{b}} \mathrm{kg} / \mathrm{inh}$ abitant/year; Data on population statistics and density areas, and number of visitors are provided in Table S1-3.

Table 4: Results of testing for trend in waste generation using the seasonal Kendall test for trend:

\begin{tabular}{|c|c|c|c|c|c|c|c|}
\hline \multirow{2}{*}{ Areas } & \multirow{2}{*}{$\mathrm{DF}^{\mathrm{a}}$} & \multicolumn{2}{|c|}{ Test Statistics } & \multicolumn{2}{|c|}{ P-values } & \multicolumn{2}{|c|}{$\begin{array}{c}\text { 95\% Confidence } \\
\text { Interval }^{\mathrm{i}}\end{array}$} \\
\hline & & Chi-Square (Het) ${ }^{\mathrm{b}}$ & $\mathrm{z}\left(\right.$ Trend) ${ }^{\mathrm{c}}$ & $\begin{array}{c}\text { Chi-Square } \\
{\text { (Het })^{\mathrm{d}}}^{\text {Con }}\end{array}$ & $\mathrm{z}(\text { Trend })^{\mathrm{f}}$ & $\mathrm{LCL}^{\mathrm{g}}$ & $\mathrm{UCL}^{\mathrm{h}}$ \\
\hline Central Zealand & 11 & 6.940 & 1.604 & 0.804 & 0.109 & -0.033 & 0.270 \\
\hline Djursland & 11 & 19.030 & -0.390 & 0.061 & 0.696 & -0.098 & 0.054 \\
\hline
\end{tabular}

${ }^{a}$ Degree of freedom; ${ }^{b}$ chi-square test for heterogeneity;

${ }^{c}$ Test statistics value for trend

${ }^{d} p$-value for chi-square test for heterogeneity indicating if the trend is different for different seasons;

${ }^{f}$-value associated with seasonal Kendall test for trend ( $p>0.05$ signifies that the trend is not statistically significant)

${ }^{g}$ Lower bound confidence interval;

${ }^{h}$ Higher bound confidence interval

${ }^{i}$ The two sided $95 \%$ confidence interval for the trend(e.g. Djursland 95\% confidence interval of estimated trend was [-0.09; 0.05]) 


\section{Temporal and geographical patterns of solid waste collected at recycling centres}

Maklawe Essonanawe Edjabou*, Giorgia Faraca, Alessio Boldrin, and Thomas Fruergaard Astrup

Department of Environmental Engineering, Technical University of Denmark, 2800 Kgs. Lyngby, Denmark

*) Corresponding author: vine@env.dtu.dk;

Phone number: +45 45251498 


\section{Figure captions}

Figure 1: Map of Denmark showing the population per municipality, 36 household waste recycling centres (HWRC) involved in this study from which seven were selected to evaluate the effectiveness of the black plastic bag policy. "Sorting WS" are HWRCs where small miscellaneous combustible (SMC) was sampled and characterised.

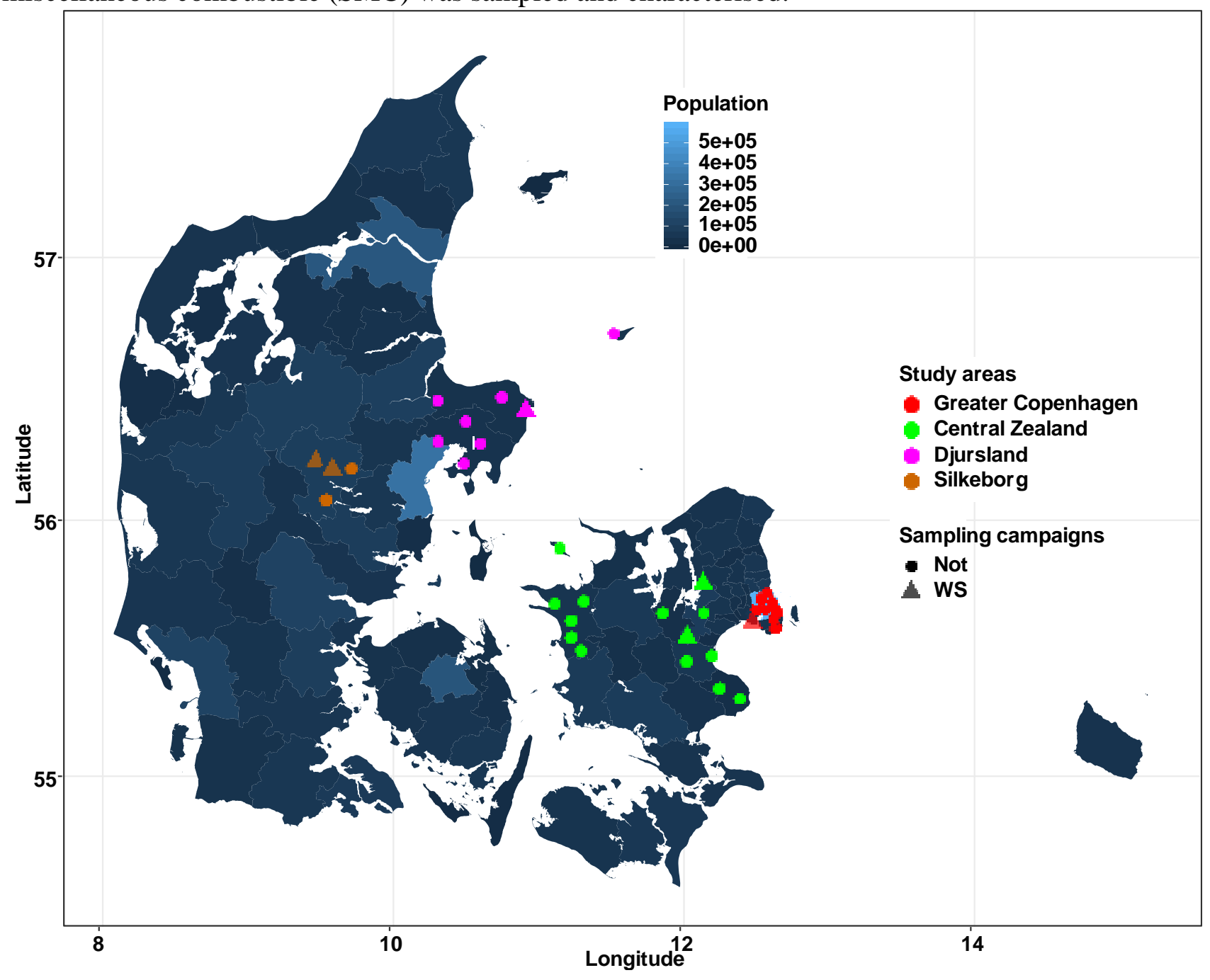


Figure 2: Total yearly waste generation [1000 Mg/year] in each study region from 2010 to 2016

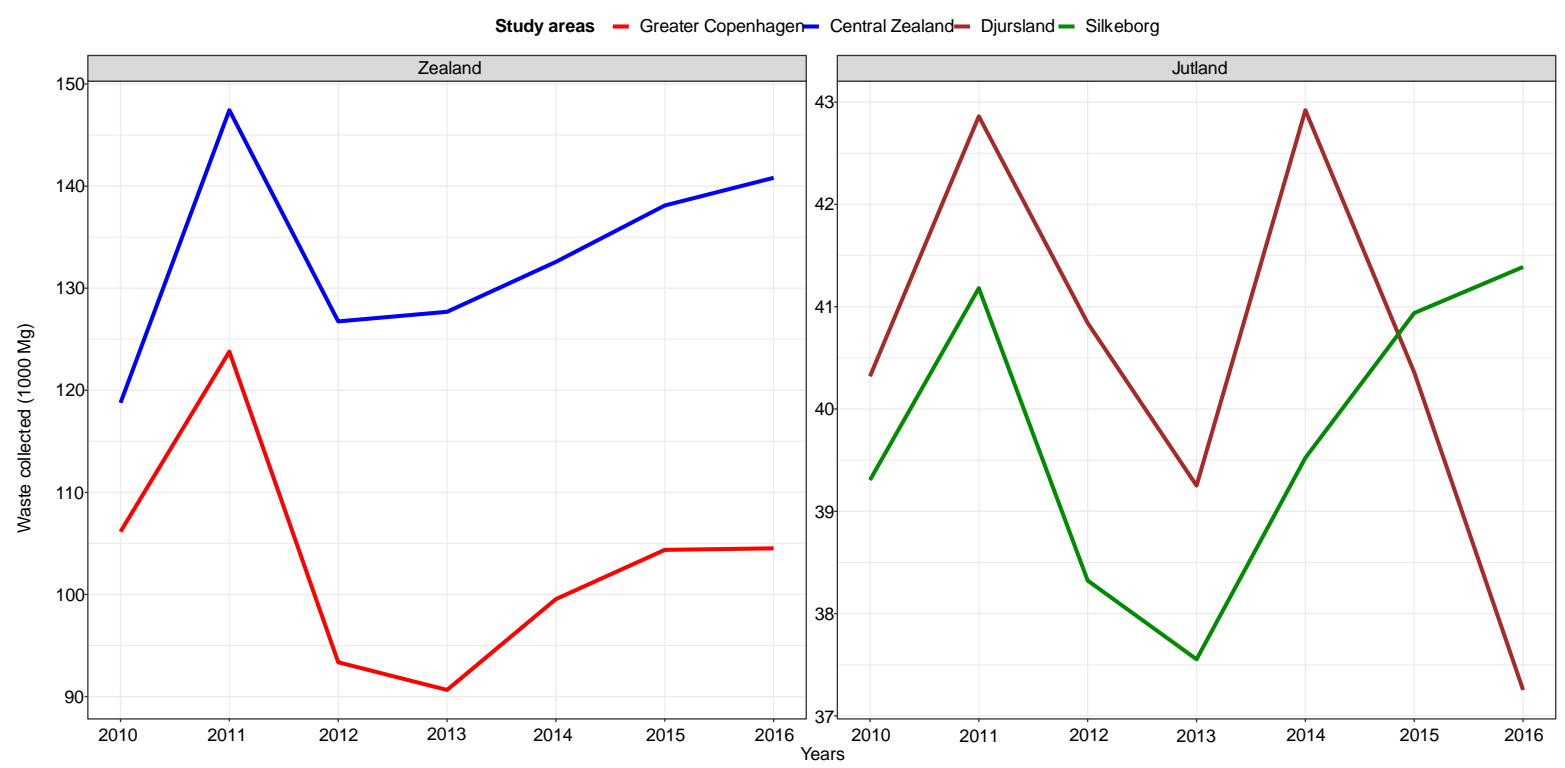


Figure 3: Decomposition of waste generation on a monthly basis in the four studies areas: Greater Copenhagen, Central Zealand, Silkeborg and Djursland.
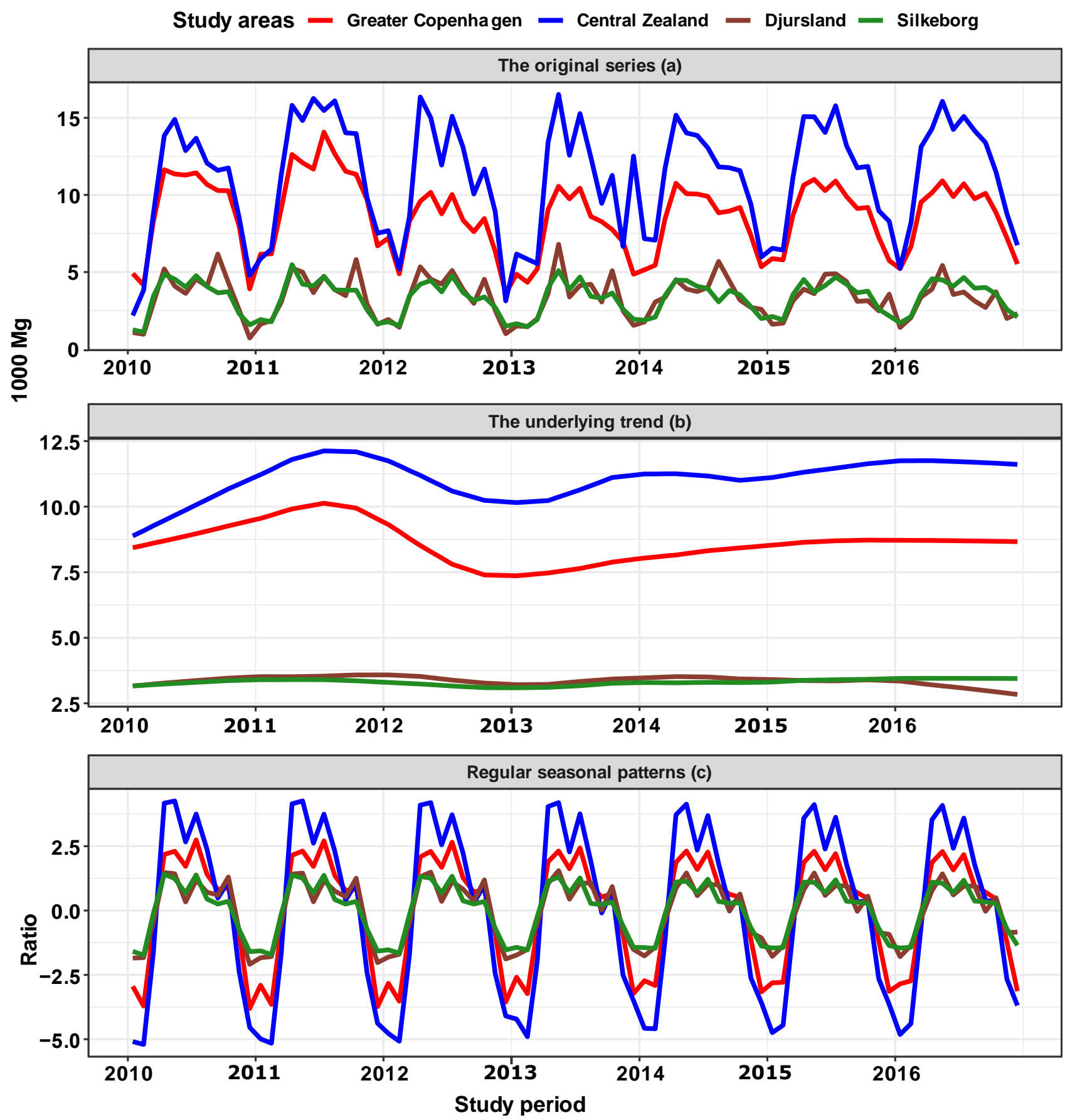
Figure 4: Boxplot showing the percentage change in mass of waste collected in and number of visitors for the period 2010-2016 (see Table S4-5 for detailed data)

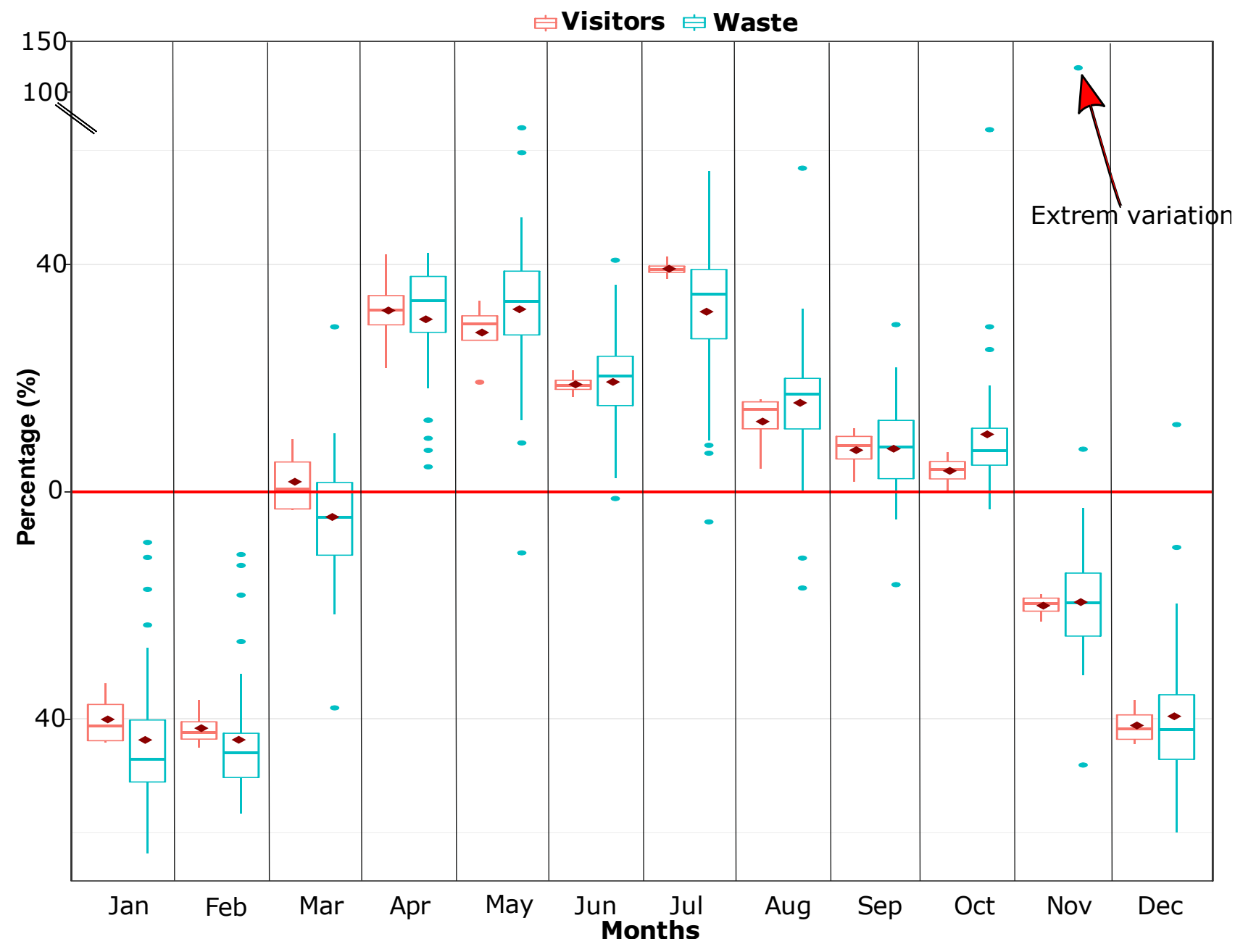


Figure 5:clr-biplot analysis of main categories and detailed target waste fractions. Dots represents the yearly waste composition. The proportion of the total variability retained by the first $\left(1^{\text {st }}\right)$ and second $\left(2^{\text {nd }}\right)$ equalled to $85 \%$, indicating that the representation of the composition was significant.

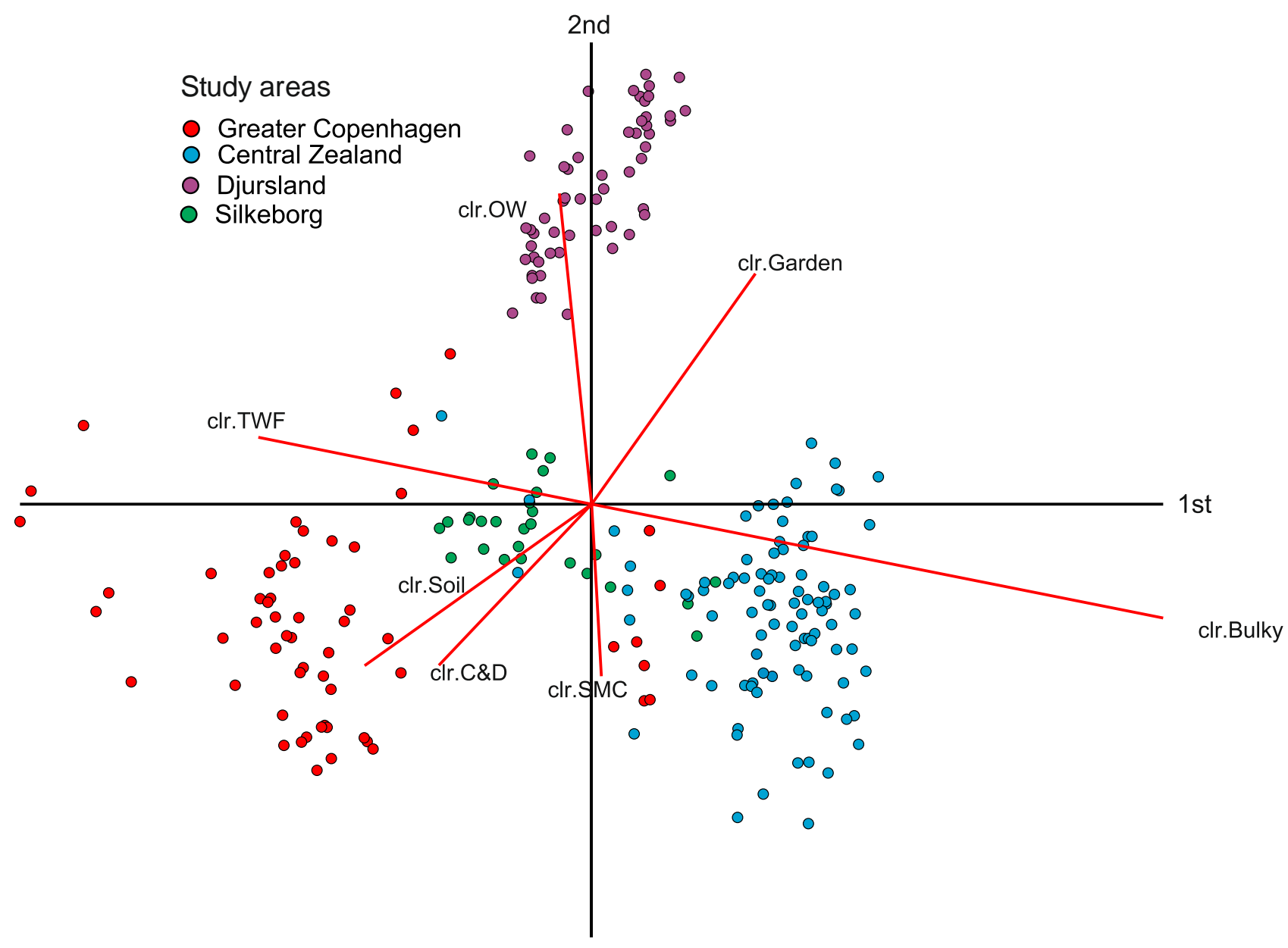


Figure 6: Bar plot showing the distribution of treatment forms of waste in Greater Copenhagen, Central Zealand, Silkeborg and Djursland from 2010 to 2016.

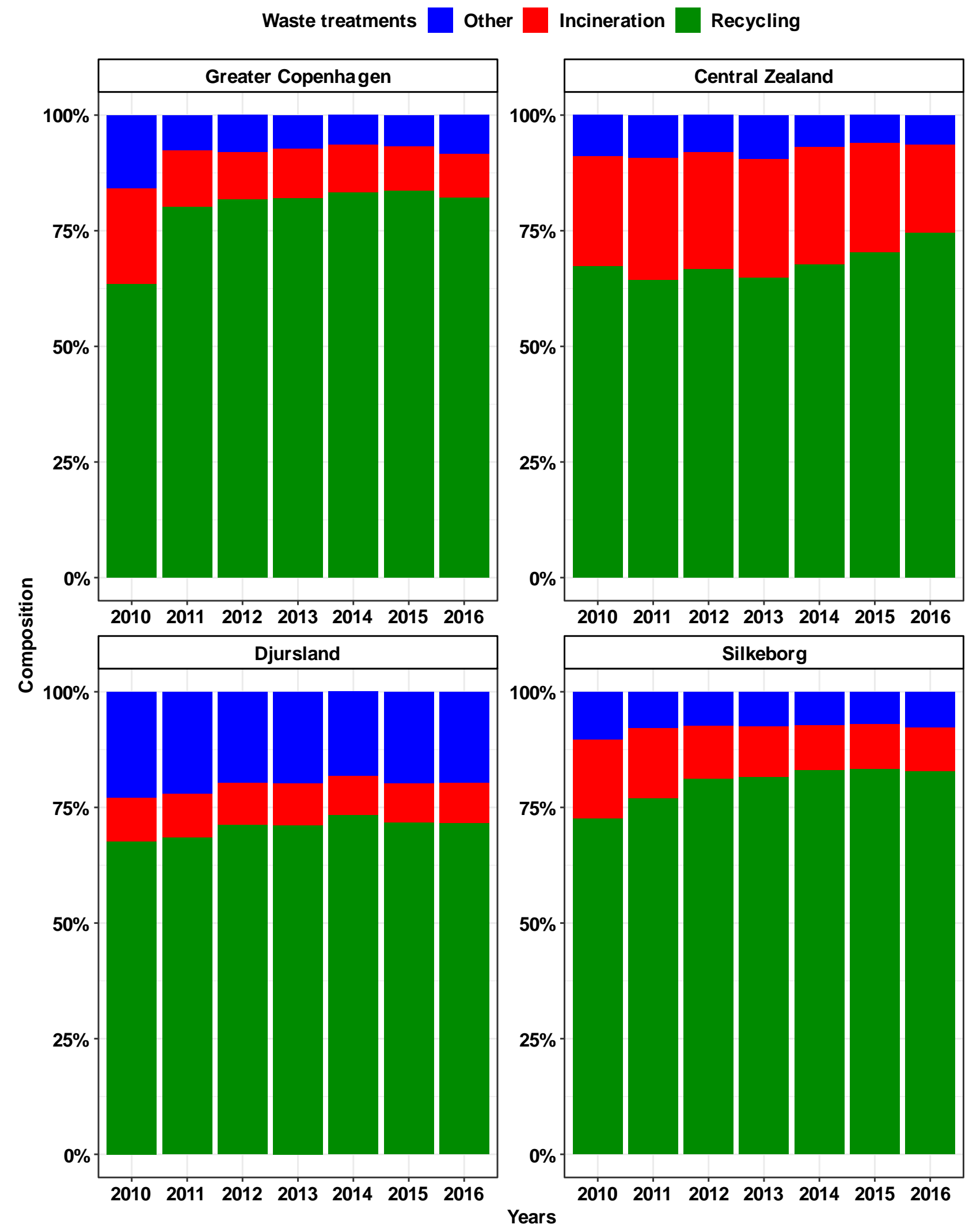


Figure 7: Geometric bar plot exploring the relative changes in treatment forms in Copenhagen, Central Zealand, Silkeborg and Djursland from 2010 to 2016

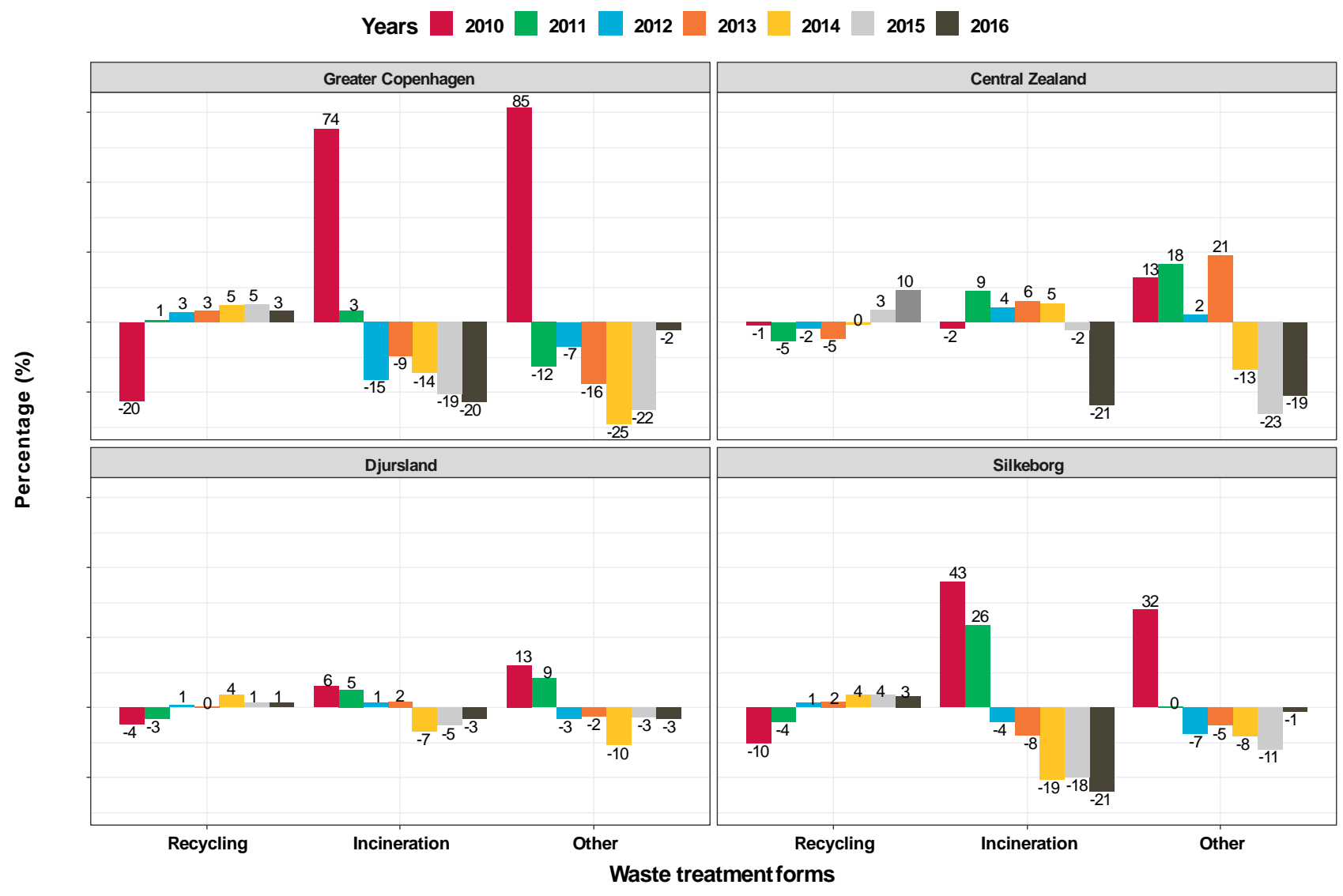


Figure 8: Ternary plot exploring the distribution between waste treatment forms. Dots present treatment forms in each HWRC from 2010 to 2016

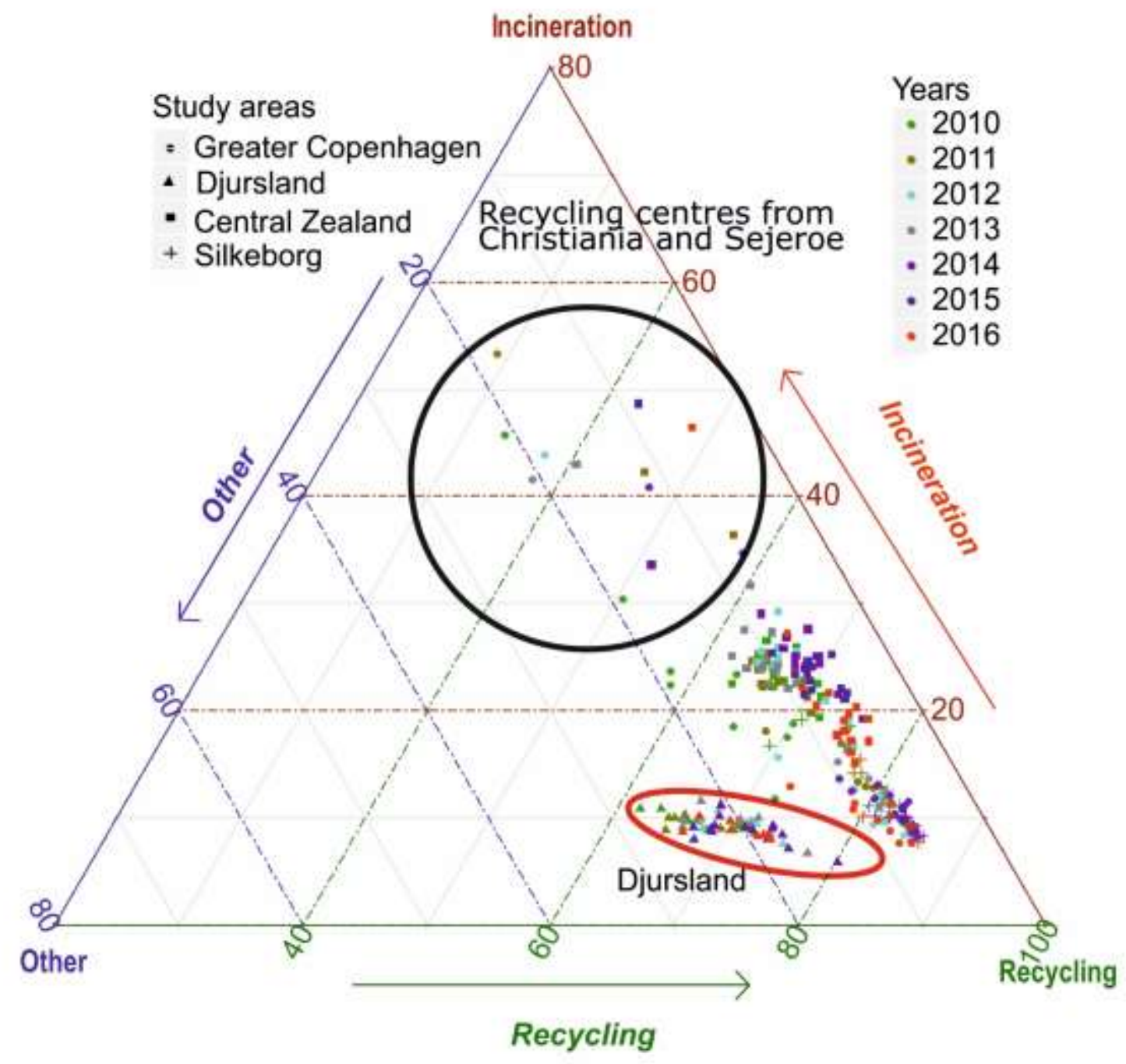


Figure 9: Composition of miscellaneous combustible from HWRC sorted according to delivery bags including mixed, clear, and black plastic bags as well as without bag. Waste sorted into target fractions, miscellaneous combustible and other (Table S14 for detailed descriptive statistics).

Main categories:

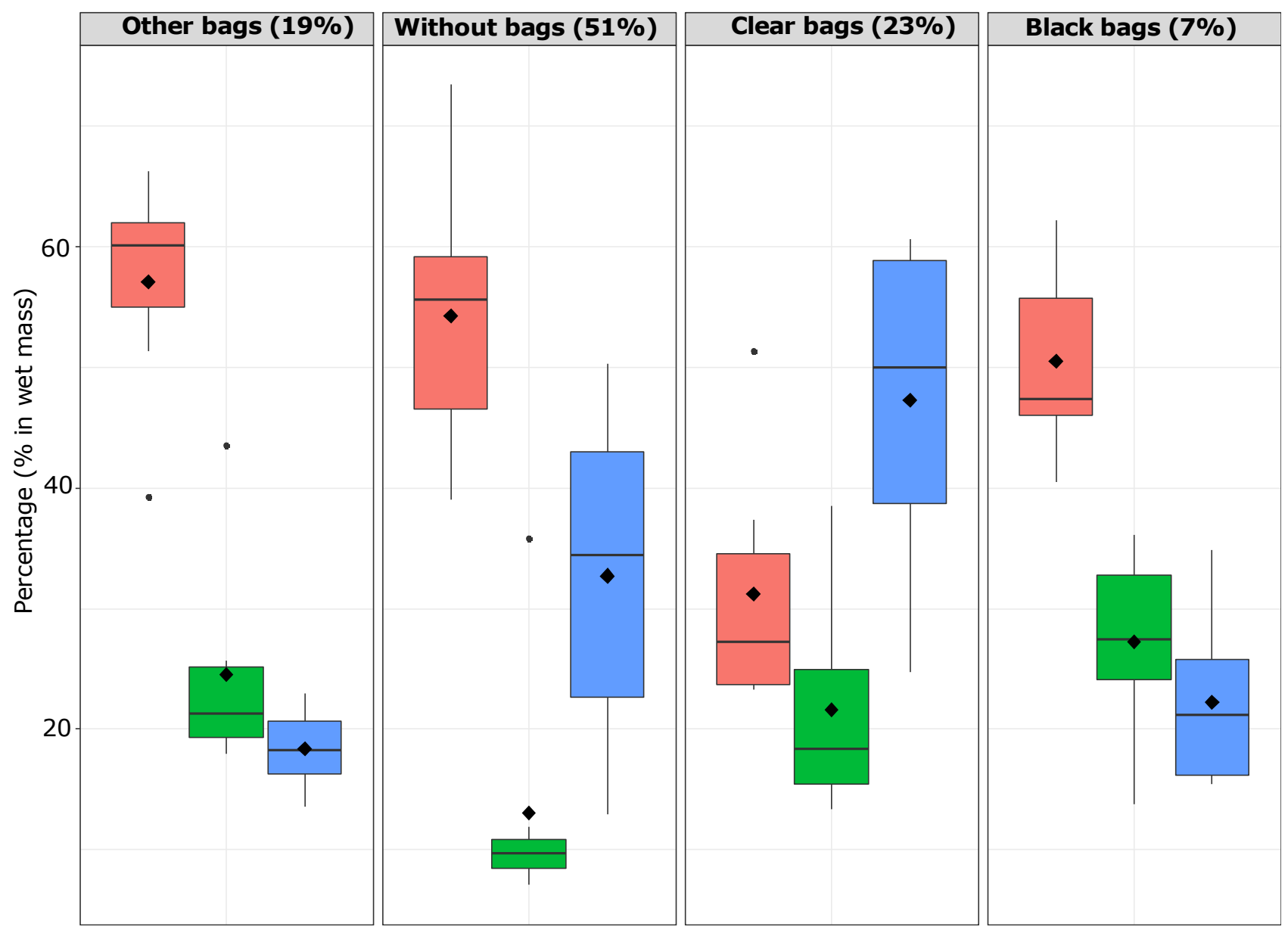


Figure 10: Ternary plot showing waste treatment forms in period 2015-2016 and expected potential if miscellaneous waste is sorted correctly at HWRCs marked as "Sorting WS" in Figure 1.

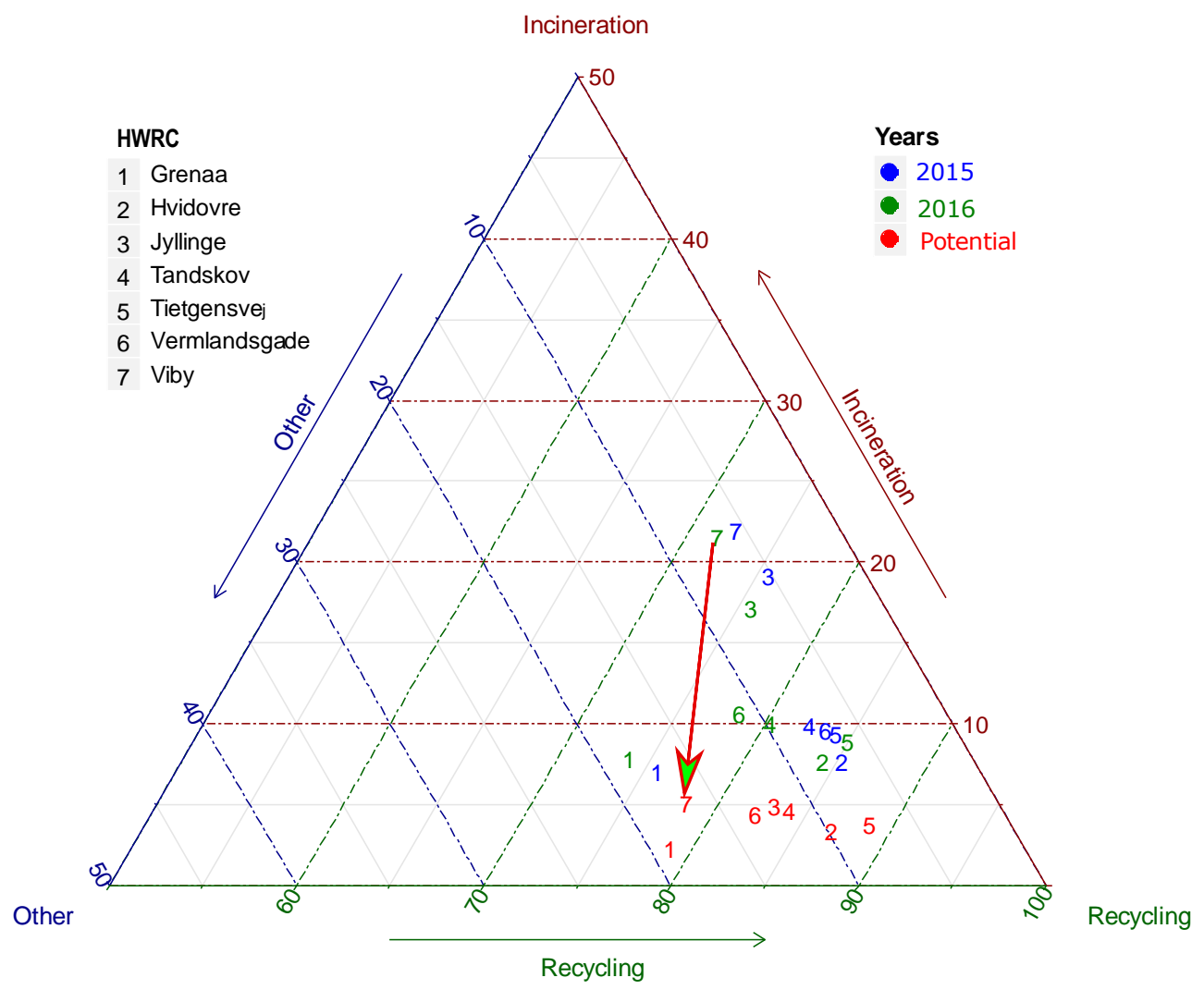


Intended for submission in Journal of environmental management

\section{Supplementary materials for:}

\section{Temporal and geographical patterns of solid waste collected at recycling centres}

Maklawe Essonanawe Edjabou*, Giorgia Faraca, Alessio Boldrin, and Thomas Fruergaard Astrup

Department of Environmental Engineering, Technical University of Denmark, 2800 Kgs. Lyngby, Denmark

*) Corresponding author: vine@env.dtu.dk;

Phone number: +45 45251498 


\section{List of Tables}

Table S1: Percentage of single-family houses and people living in single-family houses

\begin{tabular}{|c|c|c|c|c|c|c|c|c|c|}
\hline Kommune & Factor & 2010 & 2011 & 2012 & 2013 & 2014 & 2015 & 2016 & 2017 \\
\hline Frederiksberg & Houses SF & 97 & 97 & 97 & 97 & 97 & 97 & 97 & 97 \\
\hline Frederiksberg & Person SF & 95 & 95 & 95 & 95 & 95 & 95 & 95 & 95 \\
\hline Dragoer & Houses SF & 22 & 22 & 23 & 22 & 22 & 22 & 22 & 22 \\
\hline Dragoer & Person SF & 16 & 16 & 16 & 15 & 15 & 15 & 15 & 15 \\
\hline Hvidovre & Houses SF & 57 & 57 & 57 & 57 & 57 & 57 & 57 & 57 \\
\hline Hvidovre & Person SF & 46 & 46 & 46 & 46 & 47 & 46 & 46 & 46 \\
\hline Koebenhavn & Houses SF & 92 & 92 & 92 & 92 & 92 & 92 & 92 & 92 \\
\hline Koebenhavn & Person SF & 88 & 88 & 88 & 88 & 88 & 88 & 88 & 88 \\
\hline Taarnby & Houses SF & 53 & 53 & 53 & 53 & 53 & 53 & 53 & 53 \\
\hline Taarnby & Person SF & 43 & 43 & 43 & 43 & 43 & 43 & 43 & 43 \\
\hline Koege & Houses SF & 30 & 30 & 30 & 31 & 31 & 31 & 31 & 31 \\
\hline Koege & Person SF & 24 & 24 & 24 & 24 & 24 & 25 & 25 & 25 \\
\hline Kalundborg & Houses SF & 17 & 17 & 18 & 17 & 18 & 18 & 18 & 18 \\
\hline Kalundborg & Person SF & 13 & 13 & 13 & 13 & 13 & 13 & 13 & 14 \\
\hline Lejre & Houses SF & 3 & 3 & 3 & 3 & 3 & 3 & 3 & 4 \\
\hline Lejre & Person SF & 2 & 2 & 2 & 2 & 3 & 3 & 3 & 3 \\
\hline Stevns & Houses SF & 6 & 6 & 6 & 7 & 7 & 7 & 7 & 8 \\
\hline Stevns & Person SF & 5 & 5 & 5 & 5 & 5 & 5 & 6 & 6 \\
\hline Roskilde & Houses SF & 38 & 38 & 38 & 38 & 38 & 38 & 38 & 38 \\
\hline Roskilde & Person SF & 28 & 29 & 29 & 29 & 29 & 29 & 29 & 28 \\
\hline Syddjurs & Houses SF & 11 & 12 & 12 & 12 & 12 & 12 & 12 & 12 \\
\hline Syddjurs & Person SF & 7 & 8 & 8 & 8 & 8 & 8 & 8 & 8 \\
\hline Norddjurs & Houses SF & 17 & 18 & 18 & 18 & 18 & 19 & 19 & 19 \\
\hline Norddjurs & Person SF & 12 & 12 & 12 & 13 & 13 & 13 & 13 & 14 \\
\hline Silkeborg & Houses SF & 26 & 26 & 26 & 26 & 27 & 27 & 27 & 27 \\
\hline Silkeborg & Person SF & 17 & 17 & 18 & 18 & 18 & 18 & 19 & 19 \\
\hline
\end{tabular}


Table S2: Estimated number of visitors per household and population for all municipalities

\begin{tabular}{|c|c|c|c|c|c|c|c|c|c|}
\hline Regions & Municipalities & Number of visitors per & 2010 & 2011 & 2012 & 2013 & 2014 & 2015 & 2016 \\
\hline \multirow{9}{*}{$\begin{array}{c}\text { Greater } \\
\text { Copenhagen }\end{array}$} & Frederiksberg & Household & - & - & 0.43 & 1.59 & 3.1 & 3.54 & 3.42 \\
\hline & Frederiksberg & Population & - & - & 0.23 & 0.83 & 1.6 & 1.82 & 1.74 \\
\hline & Dragoer & Household & 21.58 & 23.10 & 21.93 & 21.56 & 23.25 & 21.44 & 21.68 \\
\hline & Dragoer & Population & 9.25 & 9.86 & 9.38 & 9.15 & 9.9 & 9.18 & 9.22 \\
\hline & Hvidovre & Household & 4.64 & 5.22 & 3.79 & 3.63 & 4 & 3.98 & 3.96 \\
\hline & Hvidovre & Population & 2.20 & 2.46 & 1.78 & 1.7 & 1.85 & 1.83 & 1.81 \\
\hline & Koebenhavn & Population & 0.81 & 0.87 & 0.61 & 0.54 & 0.66 & 0.71 & 0.69 \\
\hline & Taarnby & Household & 13.27 & 14.52 & 12.17 & 11.72 & 13.19 & 13.97 & 14.05 \\
\hline & Taarnby & Population & 6.13 & 6.67 & 5.56 & 5.32 & 5.94 & 6.25 & 6.25 \\
\hline \multirow{9}{*}{ Central Zealand } & Koege & Household & - & - & 6.2 & 8 & 8.36 & 8.22 & 7.31 \\
\hline & Koege & Population & - & - & 2.7 & 3.5 & 3.66 & 3.61 & 3.19 \\
\hline & Kalundborg & Household & - & - & 9.11 & 13.09 & 14.04 & 15.1 & 13.65 \\
\hline & Lejre & Household & - & - & 5.76 & 8.69 & 9.08 & 10.37 & 11 \\
\hline & Lejre & Population & - & - & 2.33 & 3.52 & 3.69 & 4.21 & 4.47 \\
\hline & Stevns & Household & - & - & 5.45 & 6.3 & 7.62 & 9.67 & 9.59 \\
\hline & Stevns & Population & - & - & 2.4 & 2.8 & 3.41 & 4.33 & 4.3 \\
\hline & Roskilde & Household & - & - & 6.97 & 8.52 & 8.38 & 9.87 & 10.1 \\
\hline & Roskilde & Population & - & - & 3.19 & 3.9 & 3.84 & 4.51 & 4.6 \\
\hline \multirow{4}{*}{ Djursland } & Syddjurs & Household & 11.23 & 12.26 & 12.04 & 12.08 & 12.33 & 12.81 & 13.35 \\
\hline & Syddjurs & Population & 4.98 & 5.42 & 5.33 & 5.38 & 5.54 & 5.8 & 6.04 \\
\hline & Norddjurs & Household & 10.63 & 11.66 & 10.95 & 10.67 & 11.04 & 11.4 & 9.49 \\
\hline & Norddjurs & Population & 4.95 & 5.46 & 5.16 & 5.06 & 5.28 & 5.5 & 4.61 \\
\hline \multirow{2}{*}{ Silkeborg } & Silkeborg & Household & 10.29 & 10.91 & 10.55 & 10.6 & 11.31 & 11.55 & 11.03 \\
\hline & Silkeborg & Population & 4.52 & 4.79 & 4.62 & 4.66 & 4.99 & 5.1 & 4.89 \\
\hline
\end{tabular}


Table S3: Aggregated yearly waste generation rates (wet mass), expressed in kg/household/year for WCRhouse,

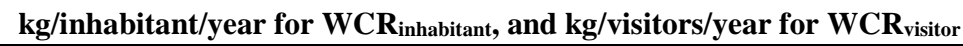

\begin{tabular}{|c|c|c|c|c|c|c|c|c|c|}
\hline \multirow{2}{*}{ Regions } & \multirow{2}{*}{ Municipalities } & \multirow{2}{*}{ Parameter } & \multicolumn{7}{|c|}{ Years } \\
\hline & & & 2010 & 2011 & 2012 & 2013 & 2014 & 2015 & 2016 \\
\hline \multirow{15}{*}{$\begin{array}{l}\text { Greater } \\
\text { Copenhagen }\end{array}$} & \multirow{3}{*}{ Frederiksberg } & $\mathrm{WCR}_{\text {house }}$ & & & 44 & 178 & 273 & 283 & 291 \\
\hline & & $\mathrm{WCR}_{\text {inhabitant }}$ & & & 23 & 93 & 141 & 146 & 148 \\
\hline & & $\mathrm{WCR}_{\text {visitor }}$ & & & 101 & 112 & 88 & 80 & 85 \\
\hline & \multirow{3}{*}{ Dragoer } & $\mathrm{WCR}_{\text {house }}$ & 1586 & 1681 & 1374 & 1392 & 1611 & 1548 & 1481 \\
\hline & & $\mathrm{WCR}_{\text {inhabitant }}$ & 680 & 717 & 587 & 591 & 686 & 663 & 630 \\
\hline & & $\mathrm{WCR}_{\text {visitor }}$ & 73 & 73 & 63 & 65 & 69 & 72 & 68 \\
\hline & \multirow{3}{*}{ Hvidovre } & $\mathrm{WCR}_{\text {house }}$ & 805 & 883 & 608 & 547 & 593 & 612 & 615 \\
\hline & & $\mathrm{WCR}_{\text {inhabitant }}$ & 382 & 416 & 285 & 255 & 275 & 282 & 281 \\
\hline & & $\mathrm{WCR}_{\text {visitor }}$ & 174 & 169 & 161 & 151 & 148 & 154 & 155 \\
\hline & \multirow{3}{*}{ Copenahgen } & $\mathrm{WCR}_{\text {house }}$ & 175 & 214 & 150 & 122 & 124 & 129 & 127 \\
\hline & & $\mathrm{WCR}_{\text {inhabitant }}$ & 93 & 113 & 78 & 63 & 63 & 65 & 64 \\
\hline & & $\mathrm{WCR}_{\text {visitor }}$ & 115 & 130 & 128 & 117 & 95 & 92 & 93 \\
\hline & \multirow{3}{*}{ Taarnby } & $\mathrm{WCR}_{\text {house }}$ & 1602 & 1793 & 1425 & 1371 & 1400 & 1517 & 1522 \\
\hline & & $\mathrm{WCR}_{\text {inhabitant }}$ & 740 & 823 & 651 & 622 & 631 & 679 & 677 \\
\hline & & $\mathrm{WCR}_{\text {visitor }}$ & 121 & 124 & 117 & 117 & 106 & 109 & 108 \\
\hline \multirow{15}{*}{ Central Zealand } & \multirow{3}{*}{ Koege } & $\mathrm{WCR}_{\text {house }}$ & 963 & 1259 & 1096 & 1106 & 1154 & 1156 & 1251 \\
\hline & & $\mathrm{WCR}_{\text {inhabitant }}$ & 418 & 547 & 477 & 484 & 506 & 508 & 546 \\
\hline & & $\mathrm{WCR}_{\text {visitor }}$ & & & 177 & 138 & 138 & 141 & 171 \\
\hline & \multirow{3}{*}{ Kalundborg } & $\mathrm{WCR}_{\text {house }}$ & 1576 & 1681 & 1477 & 1403 & 1559 & 1563 & 1454 \\
\hline & & $\mathrm{WCR}_{\text {inhabitant }}$ & 727 & 778 & 690 & 660 & 735 & 740 & 689 \\
\hline & & $\mathrm{WCR}_{\text {visitor }}$ & & & 162 & 107 & 111 & 104 & 106 \\
\hline & \multirow{3}{*}{ Lejre } & $\mathrm{WCR}_{\text {house }}$ & 1079 & 1294 & 1144 & 1268 & 1135 & 1148 & 1207 \\
\hline & & $\mathrm{WCR}_{\text {inhabitant }}$ & 430 & 520 & 462 & 514 & 461 & 466 & 491 \\
\hline & & $\mathrm{WCR}_{\text {visitor }}$ & & & 198 & 146 & 125 & 111 & 110 \\
\hline & \multirow{3}{*}{ Stevns } & $\mathrm{WCR}_{\text {house }}$ & 1203 & 1461 & 1220 & 1243 & 965 & 963 & 1038 \\
\hline & & $\mathrm{WCR}_{\text {inhabitant }}$ & 523 & 641 & 537 & 553 & 431 & 431 & 466 \\
\hline & & $\mathrm{WCR}_{\text {visitor }}$ & & & 224 & 197 & 127 & 100 & 108 \\
\hline & \multirow{3}{*}{ Roskilde } & $\mathrm{WCR}_{\text {house }}$ & 1002 & 1357 & 1128 & 1128 & 1215 & 1320 & 1332 \\
\hline & & $\mathrm{WCR}_{\text {inhabitant }}$ & 458 & 622 & 517 & 517 & 557 & 603 & 607 \\
\hline & & $\mathrm{WCR}_{\text {visitor }}$ & & & 162 & 132 & 145 & 134 & 132 \\
\hline \multirow{6}{*}{ Djursland } & \multirow{3}{*}{ Syddjurs } & $\mathrm{WCR}_{\text {house }}$ & 1054 & 1109 & 998 & 965 & 1039 & 1005 & 1063 \\
\hline & & $\mathrm{WCR}_{\text {inhabitant }}$ & 468 & 491 & 442 & 430 & 467 & 455 & 481 \\
\hline & & $\mathrm{WCR}_{\text {visitor }}$ & 94 & 91 & 83 & 80 & 84 & 78 & 80 \\
\hline & \multirow{3}{*}{ Norddjurs } & $\mathrm{WCR}_{\text {house }}$ & 1204 & 1280 & 1275 & 1199 & 1310 & 1188 & 941 \\
\hline & & $\mathrm{WCR}_{\text {inhabitant }}$ & 560 & 600 & 602 & 569 & 627 & 573 & 456 \\
\hline & & $\mathrm{WCR}_{\text {visitor }}$ & 113 & 110 & 117 & 112 & 119 & 104 & 99 \\
\hline \multirow{3}{*}{ Silkeborg } & \multirow{3}{*}{ Silkeborg } & $\mathrm{WCR}_{\text {house }}$ & 1020 & 1066 & 988 & 963 & 1008 & 1037 & 1035 \\
\hline & & $\mathrm{WCR}_{\text {inhabitant }}$ & 448 & 467 & 433 & 424 & 444 & 458 & 459 \\
\hline & & $\mathrm{WCR}_{\text {visitor }}$ & 99 & 98 & 94 & 91 & 89 & 90 & 94 \\
\hline
\end{tabular}

${ }^{\mathrm{a}} \mathrm{kg} / \mathrm{visitor} / \mathrm{year}$; ${ }^{\mathrm{kg}} / \mathrm{inhabitant/year;} \mathrm{Data} \mathrm{on} \mathrm{population} \mathrm{statistics} \mathrm{and} \mathrm{density} \mathrm{areas,} \mathrm{and} \mathrm{number} \mathrm{of} \mathrm{visitors} \mathrm{are} \mathrm{provided} \mathrm{in} \mathrm{Table} \mathrm{S1-3}$ 
Table S4: Mass of waste annually collected [Mg/year wet mass] at individual HWRCs

\begin{tabular}{|c|c|c|c|c|c|c|c|c|c|c|}
\hline \multirow{2}{*}{ Areas } & \multirow{2}{*}{ Municipalities } & & \multirow{2}{*}{ HWRC } & \multicolumn{7}{|c|}{ Mass of waste collected [Mg/year] } \\
\hline & & & & 2010 & 2011 & 2012 & 2013 & 2014 & 2015 & 2016 \\
\hline \multirow{10}{*}{$\begin{array}{l}\text { Greater } \\
\text { Copenha } \\
\text { gen }\end{array}$} & Frederiksberg & 1 & Bispeengen & 0 & 0 & 2277 & 9334 & 14344 & 14897 & 15339 \\
\hline & Dragoer & 2 & Dragoer & 9136 & 9757 & 7993 & 8177 & 9522 & 9200 & 8808 \\
\hline & Hvidovre & 3 & Hvidovre & 18864 & 20647 & 14317 & 13008 & 14141 & 14621 & 14720 \\
\hline & \multirow{5}{*}{ Copenhagen } & 4 & Borgervaenget & 1749 & 5097 & 6197 & 5886 & 5701 & 6249 & 6613 \\
\hline & & 5 & Chrisiania & 519 & 543 & 589 & 764 & 706 & 998 & 998 \\
\hline & & 6 & Kulbanevej & 21995 & 25474 & 17691 & 13148 & 12106 & 12782 & 13670 \\
\hline & & 7 & Vasbygade & 3977 & 4285 & 2391 & 2152 & 2558 & 2437 & 2375 \\
\hline & & 8 & Vermlandsgade & 20347 & 24756 & 15500 & 12684 & 14439 & 14784 & 13494 \\
\hline & \multirow[t]{2}{*}{ Taarnby } & 9 & Kirstinehoej & 28585 & 32307 & 25599 & 24578 & 25099 & 27398 & 27549 \\
\hline & & 10 & Vaegtergangen & 984 & 923 & 806 & 921 & 932 & 1005 & 955 \\
\hline \multirow{14}{*}{$\begin{array}{l}\text { Central } \\
\text { Zealand }\end{array}$} & \multirow{6}{*}{ Kalundborg } & 11 & Bregninge & 5585 & 6438 & 6312 & 6536 & 7448 & 7065 & 6831 \\
\hline & & 12 & Goerlev & 6772 & 7413 & 6655 & 6799 & 6961 & 6487 & 6408 \\
\hline & & 13 & Hoeng & 3908 & 4114 & 3321 & 3454 & 4030 & 4330 & 4194 \\
\hline & & 14 & Kalundborg & 16280 & 17116 & 14339 & 12624 & 14074 & 15110 & 13491 \\
\hline & & 15 & Sejeroe & 472 & 339 & 619 & 264 & 285 & 288 & 291 \\
\hline & & 16 & Ubby & 2513 & 2578 & 2132 & 2055 & 2582 & 2411 & 2162 \\
\hline & \multirow[t]{2}{*}{ Koege } & 17 & Bjaeverskov & 4364 & 5414 & 4248 & 4720 & 4457 & 5068 & 6784 \\
\hline & & 18 & Koege & 19145 & 25514 & 22669 & 22848 & 24670 & 24647 & 25443 \\
\hline & Lejre & 19 & Torkilstrup & 11330 & 13743 & 12250 & 13655 & 12300 & 12496 & 13209 \\
\hline & \multirow{3}{*}{ Roskilde } & 20 & Jyllinge & 7036 & 7575 & 7628 & 7599 & 8731 & 10371 & 9926 \\
\hline & & 21 & Roskilde & 23430 & 35210 & 27502 & 27287 & 28697 & 30077 & 30769 \\
\hline & & 22 & Viby & 6672 & 8215 & 7544 & 7945 & 9023 & 10362 & 11065 \\
\hline & \multirow[t]{2}{*}{ Stevns } & 23 & Haarlev & 5303 & 6572 & 6281 & 6811 & 4032 & 3869 & 4651 \\
\hline & & 24 & Store Hedding & 5961 & 7196 & 5245 & 5092 & 5288 & 5519 & 5588 \\
\hline \multirow{8}{*}{ Djursland } & \multirow{4}{*}{ Norddjurs } & 25 & Glesborg & 4126 & 4491 & 4141 & 3967 & 4063 & 4097 & 4185 \\
\hline & & 26 & Grenaa & 9107 & 9869 & 10765 & 10468 & 12139 & 9854 & 9232 \\
\hline & & 27 & Roende & 5084 & 5132 & 4797 & 4294 & 4673 & 4796 & 2361 \\
\hline & & 28 & Tirstrup & 2842 & 3075 & 2836 & 2677 & 2695 & 2810 & 1483 \\
\hline & \multirow{4}{*}{ Syddjurs } & 29 & Ebeltoft & 6255 & 6618 & 5994 & 5816 & 6365 & 6206 & 6556 \\
\hline & & 30 & Hornslet & 5187 & 5441 & 4979 & 4819 & 5372 & 5157 & 5760 \\
\hline & & 31 & Knebel & 3503 & 3706 & 3212 & 3169 & 3296 & 3219 & 3285 \\
\hline & & 32 & Ryomgaard & 4216 & 4529 & 4118 & 4042 & 4318 & 4220 & 4393 \\
\hline \multirow{4}{*}{ Silkeborg } & \multirow{4}{*}{ Silkeborg } & 33 & Gjern & 3838 & 4247 & 4048 & 4027 & 4306 & 4346 & 4580 \\
\hline & & 34 & Tandskov & 16114 & 17615 & 16438 & 15660 & 16169 & 17028 & 16805 \\
\hline & & 35 & Them & 4303 & 4489 & 4187 & 4044 & 4859 & 4913 & 5375 \\
\hline & & 36 & Tietgensvej & 15052 & 14831 & 13651 & 13823 & 14188 & 14651 & 14629 \\
\hline
\end{tabular}


Table S5: Number of person per household; number of household for single and multi-family houses

\begin{tabular}{|c|c|c|c|c|c|c|c|c|c|}
\hline Municipalities & Number of person in & 2010 & 2011 & 2012 & 2013 & 2014 & 2015 & 2016 & 2017 \\
\hline Frederiksberg & Household & 1.86 & 1.88 & 1.9 & 1.92 & 1.94 & 1.95 & 1.97 & 1.98 \\
\hline Frederiksberg & MF household & 1.82 & 1.85 & 1.86 & 1.89 & 1.91 & 1.92 & 1.93 & 1.94 \\
\hline Frederiksberg & SF household & 3.07 & 3.09 & 3.07 & 3.1 & 3.13 & 3.15 & 3.16 & 3.17 \\
\hline Dragoer & Household & 2.33 & 2.35 & 2.34 & 2.37 & 2.36 & 2.35 & 2.36 & 2.38 \\
\hline Dragoer & MF household & 1.64 & 1.64 & 1.61 & 1.63 & 1.64 & 1.6 & 1.61 & 1.63 \\
\hline Dragoer & SF household & 2.53 & 2.55 & 2.55 & 2.58 & 2.57 & 2.57 & 2.58 & 2.59 \\
\hline Hvidovre & Household & 2.13 & 2.15 & 2.16 & 2.17 & 2.19 & 2.2 & 2.22 & 2.23 \\
\hline Hvidovre & MF household & 1.73 & 1.74 & 1.74 & 1.76 & 1.78 & 1.79 & 1.79 & 1.8 \\
\hline Hvidovre & SF household & 2.67 & 2.69 & 2.71 & 2.72 & 2.73 & 2.76 & 2.79 & 2.81 \\
\hline Copenhagen & Household & 1.88 & 1.91 & 1.94 & 1.96 & 1.98 & 2 & 2.02 & 2.03 \\
\hline Copenhagen & MF household & 1.81 & 1.83 & 1.86 & 1.88 & 1.9 & 1.92 & 1.94 & 1.95 \\
\hline Copenhagen & SF household & 2.78 & 2.8 & 2.82 & 2.85 & 2.89 & 2.91 & 2.94 & 2.96 \\
\hline Taarnby & Household & 2.17 & 2.18 & 2.19 & 2.2 & 2.22 & 2.24 & 2.25 & 2.26 \\
\hline Taarnby & MF household & 1.75 & 1.76 & 1.77 & 1.78 & 1.79 & 1.79 & 1.8 & 1.81 \\
\hline Taarnby & SF household & 2.64 & 2.65 & 2.67 & 2.7 & 2.72 & 2.75 & 2.76 & 2.78 \\
\hline Koege & Household & 2.3 & 2.3 & 2.3 & 2.29 & 2.28 & 2.28 & 2.29 & 2.29 \\
\hline Koege & MF household & 1.8 & 1.8 & 1.8 & 1.79 & 1.79 & 1.8 & 1.82 & 1.83 \\
\hline Koege & SF household & 2.52 & 2.52 & 2.52 & 2.5 & 2.5 & 2.49 & 2.5 & 2.5 \\
\hline Kalundborg & Household & 2.19 & 2.19 & 2.16 & 2.17 & 2.16 & 2.15 & 2.15 & 2.14 \\
\hline Kalundborg & MF household & 1.58 & 1.59 & 1.57 & 1.57 & 1.58 & 1.59 & 1.61 & 1.64 \\
\hline Kalundborg & SF household & 2.32 & 2.31 & 2.29 & 2.29 & 2.28 & 2.27 & 2.26 & 2.25 \\
\hline Lejre & Household & 2.55 & 2.53 & 2.53 & 2.51 & 2.51 & 2.51 & 2.51 & 2.5 \\
\hline Lejre & MF household & 1.84 & 1.86 & 1.84 & 1.84 & 1.89 & 1.86 & 1.86 & 1.84 \\
\hline Lejre & SF household & 2.57 & 2.55 & 2.55 & 2.54 & 2.53 & 2.53 & 2.53 & 2.52 \\
\hline Stevns & Household & 2.33 & 2.3 & 2.3 & 2.27 & 2.26 & 2.26 & 2.25 & 2.25 \\
\hline Stevns & MF household & 1.76 & 1.75 & 1.71 & 1.66 & 1.69 & 1.72 & 1.75 & 1.84 \\
\hline Stevns & SF household & 2.36 & 2.34 & 2.33 & 2.32 & 2.31 & 2.3 & 2.29 & 2.29 \\
\hline Roskilde & Household & 2.23 & 2.22 & 2.23 & 2.22 & 2.22 & 2.23 & 2.24 & 2.25 \\
\hline Roskilde & MF household & 1.67 & 1.67 & 1.68 & 1.68 & 1.67 & 1.68 & 1.68 & 1.69 \\
\hline Roskilde & SF household & 2.56 & 2.56 & 2.56 & 2.56 & 2.56 & 2.57 & 2.58 & 2.59 \\
\hline Syddjurs & Household & 2.28 & 2.29 & 2.29 & 2.28 & 2.26 & 2.25 & 2.24 & 2.23 \\
\hline Syddjurs & MF household & 1.47 & 1.49 & 1.51 & 1.5 & 1.51 & 1.5 & 1.51 & 1.53 \\
\hline Syddjurs & SF household & 2.39 & 2.4 & 2.39 & 2.38 & 2.36 & 2.34 & 2.34 & 2.32 \\
\hline Norddjurs & Household & 2.15 & 2.14 & 2.13 & 2.12 & 2.1 & 2.09 & 2.08 & 2.07 \\
\hline Norddjurs & MF household & 1.49 & 1.47 & 1.49 & 1.48 & 1.49 & 1.51 & 1.5 & 1.52 \\
\hline Norddjurs & SF household & 2.3 & 2.28 & 2.27 & 2.26 & 2.24 & 2.22 & 2.21 & 2.2 \\
\hline Silkeborg & Household & 2.28 & 2.29 & 2.29 & 2.28 & 2.27 & 2.27 & 2.26 & 2.25 \\
\hline Silkeborg & MF household & 1.53 & 1.53 & 1.55 & 1.55 & 1.56 & 1.57 & 1.57 & 1.58 \\
\hline Silkeborg & SF household & 2.55 & 2.55 & 2.55 & 2.54 & 2.53 & 2.52 & 2.51 & 2.5 \\
\hline
\end{tabular}


Table S6: Ratio to determine the difference in monthly waste generation in the four areas

\begin{tabular}{|c|c|c|c|c|c|c|c|c|c|c|c|c|}
\hline \multicolumn{13}{|l|}{ Month } \\
\hline & \multicolumn{3}{|c|}{ Greater Copenhagen } & \multicolumn{3}{|c|}{ Roskilde } & \multicolumn{3}{|c|}{ Djursland } & \multicolumn{3}{|c|}{ Silkeborg } \\
\hline & Tonnes/year & Month/Mean ${ }^{\mathrm{b}}$ & Ratio $^{c}$ & Tonnes/year & Month/Mean ${ }^{\mathrm{b}}$ & Ratio $^{c}$ & Tonnes/year & Month/Mean ${ }^{\mathrm{b}}$ & Ratio $^{c}$ & Tonnes/year & Month/Mean ${ }^{\mathrm{b}}$ & Ratio $^{\mathrm{c}}$ \\
\hline Jan & 6411.00 & 0.65 & -35.33 & 5834.44 & 0.53 & -47.42 & 1560.80 & 0.46 & -53.80 & 1758.47 & 0.53 & -46.91 \\
\hline $\mathrm{Feb}$ & 6085.71 & 0.61 & -38.61 & 6149.45 & 0.55 & -44.58 & 1786.45 & 0.53 & -47.13 & 1707.45 & 0.52 & -48.45 \\
\hline Mar & 9431.14 & 0.95 & -4.86 & 10018.52 & 0.90 & -9.72 & 3036.11 & 0.90 & -10.14 & 3240.12 & 0.98 & -2.17 \\
\hline Apr & 12350.71 & 1.25 & 24.59 & 14850.91 & 1.34 & 33.83 & 4526.92 & 1.34 & 33.99 & 4582.81 & 1.38 & 38.37 \\
\hline May & 12614.86 & 1.27 & 27.25 & 15197.23 & 1.37 & 36.95 & 4765.23 & 1.41 & 41.04 & 4424.31 & 1.34 & 33.58 \\
\hline June & 11886.57 & 1.20 & 19.91 & 13682.08 & 1.23 & 23.30 & 3861.84 & 1.14 & 14.30 & 4000.57 & 1.21 & 20.79 \\
\hline July & 12752.14 & 1.29 & 28.64 & 14778.88 & 1.33 & 33.18 & 4445.21 & 1.32 & 31.57 & 4594.03 & 1.39 & 38.71 \\
\hline Aug & 11423.86 & 1.15 & 15.24 & 13269.57 & 1.20 & 19.58 & 4173.94 & 1.24 & 23.54 & 3748.28 & 1.13 & 13.17 \\
\hline Sep & 10886.57 & 1.10 & 9.82 & 11718.40 & 1.06 & 5.60 & 3696.43 & 1.09 & 9.41 & 3627.29 & 1.10 & 9.52 \\
\hline Oct & 10690.57 & 1.08 & 7.84 & 11942.28 & 1.08 & 7.62 & 4258.83 & 1.26 & 26.05 & 3636.94 & 1.10 & 9.81 \\
\hline Nov & 8631.00 & 0.87 & -12.93 & 8725.73 & 0.79 & -21.37 & 2524.75 & 0.75 & -25.27 & 2583.61 & 0.78 & -21.99 \\
\hline Dec & 5795.14 & 0.58 & -41.54 & 6994.87 & 0.63 & -36.97 & 1907.27 & 0.56 & -43.55 & 1841.03 & 0.56 & -44.41 \\
\hline Mean $^{a}$ & 9913.00 & - & - & 11097.00 & - & - & 3379.00 & - & - & 3312.00 & - & - \\
\hline
\end{tabular}

${ }^{a}$ monthly mean or mean of waste from the 12 months (Mean).

${ }^{b}$ monthly mean divided by the monthly waste generation. Example: Jan for Greater Copenhagen: $6411 / 9913=0.65$

${ }^{c}$ calculated based on the value of month/mean. If month/mean is higher than 1 (e.g. April Greater Copenhagen, 1.25), then Ratio is (1.27-1)*100=27.25\%. However, if month/mean is lower than 1, (e.g. January Roskilde, 0.53), then Ratio is $-(1-0.53) * 100=-47.42$ 
Table S7: Ratio to determine the difference in monthly waste generation in the four areas

\begin{tabular}{|c|c|c|c|c|c|c|c|c|c|c|c|c|}
\hline \multirow{2}{*}{ HWRC } & \multicolumn{12}{|c|}{ Months } \\
\hline & Jan & Feb & Mar & Apr & Maj & Jun & Jul & Aug & Sep & Okt & Nov & Dec \\
\hline Tandskov & -44.2 & -44.5 & 5.2 & 42.0 & 34.6 & 20.4 & 35.8 & 6.8 & 4.9 & 6.0 & -19.6 & -47.4 \\
\hline Tietgensvej & -49.4 & -51.9 & -12.2 & 35.3 & 34.4 & 22.6 & 40.4 & 19.2 & 14.4 & 14.4 & -25.6 & -41.7 \\
\hline Bispeengen & -23.5 & -18.2 & 0.8 & 18.2 & 21.6 & 23.6 & 22.1 & -11.7 & -4.6 & 6.2 & -8.6 & -25.9 \\
\hline Bjaeverskov & -54.7 & -55.0 & -14.7 & 39.8 & 42.8 & 23.4 & 56.3 & 18.7 & 10.0 & 9.2 & -32.2 & -43.6 \\
\hline Borgervaenget & -11.6 & -13.0 & 0.4 & 7.3 & 8.6 & -1.2 & 8.1 & 0.0 & 4.1 & 9.7 & 7.5 & -19.8 \\
\hline Bregninge & -52.8 & -52.9 & 0.2 & 40.9 & 31.7 & 22.8 & 42.7 & 32.1 & 1.6 & 2.5 & -25.4 & -43.5 \\
\hline Chrisiania & -31.3 & -42.5 & 29.0 & 12.6 & 12.6 & 26.6 & 9.0 & 5.5 & 19.1 & 12.6 & -17.7 & -35.5 \\
\hline Dragoer & -49.1 & -53.5 & -11.1 & 38.5 & 44.6 & 23.8 & 34.6 & 17.1 & 21.9 & 6.0 & -19.6 & -53.3 \\
\hline Ebeltoft & -55.0 & -47.5 & 6.1 & 38.8 & 33.0 & 15.8 & 34.8 & 11.9 & 10.4 & 16.7 & -14.4 & -50.5 \\
\hline Gjern & -49.8 & -48.4 & 6.3 & 34.0 & 34.0 & 15.2 & 42.3 & 11.0 & 10.1 & 8.2 & -22.3 & -40.6 \\
\hline Glesborg & -56.0 & -48.4 & 0.8 & 34.8 & 33.6 & 15.5 & 55.2 & 22.6 & 2.7 & 10.0 & -19.6 & -51.2 \\
\hline Goerlev & -51.3 & -43.1 & -4.9 & 37.0 & 32.5 & 21.5 & 46.0 & 17.6 & 13.0 & 3.7 & -26.6 & -45.4 \\
\hline Grenaa & -63.6 & -43.3 & -38.0 & 31.0 & 64.0 & 2.3 & 6.7 & 56.9 & -4.9 & 63.7 & -48.1 & -26.7 \\
\hline Hoeng & -44.2 & -43.2 & 10.2 & 28.2 & 48.2 & 24.0 & 31.5 & 13.9 & -2.3 & -3.1 & -25.9 & -37.3 \\
\hline Hornslet & -51.0 & -53.5 & -10.1 & 26.4 & 35.7 & 19.7 & 38.6 & 10.9 & 29.4 & 18.6 & -17.2 & -47.5 \\
\hline Hvidovre & -40.0 & -43.8 & -7.5 & 30.8 & 31.9 & 25.0 & 27.9 & 21.5 & 12.4 & 6.3 & -17.4 & -47.0 \\
\hline Haarlev & -43.8 & -56.0 & -21.5 & 36.0 & 45.2 & 8.0 & 17.5 & 25.5 & 1.8 & 9.1 & -11.9 & -9.8 \\
\hline Jyllinge & -52.1 & -51.6 & -7.5 & 30.7 & 38.7 & 36.4 & 36.5 & 15.3 & 8.1 & 7.1 & -25.6 & -35.8 \\
\hline Kalundborg & -42.0 & -40.0 & 1.6 & 31.3 & 39.0 & 19.9 & 29.7 & 15.6 & 10.1 & 1.4 & -26.2 & -40.5 \\
\hline Kirstinehoej & -48.6 & -48.7 & -6.2 & 37.3 & 41.9 & 29.5 & 41.9 & 19.6 & 0.7 & 4.8 & -20.3 & -51.9 \\
\hline Knebel & -48.3 & -46.6 & -4.7 & 30.5 & 27.7 & 10.9 & 45.2 & 16.3 & 13.9 & 14.1 & -16.8 & -42.1 \\
\hline Koege & -53.5 & -44.6 & -15.9 & 38.2 & 41.2 & 19.6 & 37.2 & 18.2 & 6.7 & 9.4 & -23.2 & -33.4 \\
\hline Kulbanevej & -27.4 & -32.0 & 1.8 & 18.8 & 16.8 & 15.6 & 30.8 & 12.0 & 10.8 & 6.5 & -14.7 & -38.8 \\
\hline Roende & -46.7 & -48.9 & -4.2 & 33.2 & 32.9 & 26.3 & 31.6 & 0.6 & 15.7 & 29.0 & -12.0 & -57.4 \\
\hline Roskilde & -39.8 & -39.9 & -15.5 & 27.3 & 32.5 & 23.5 & 23.9 & 18.8 & 2.4 & 17.3 & -12.6 & -37.8 \\
\hline Ryomgaard & -46.3 & -48.7 & -2.2 & 36.0 & 28.6 & 15.0 & 37.9 & 17.1 & 13.9 & 6.0 & -12.6 & -44.8 \\
\hline Sejeroe & -47.4 & -49.8 & -14.5 & 4.4 & -10.8 & 8.7 & -5.3 & -17.0 & -16.4 & 3.0 & 133.3 & 11.8 \\
\hline Store_Hedding & -45.8 & -42.4 & -3.8 & 29.1 & 34.8 & 29.9 & 35.5 & 26.0 & 4.8 & -0.5 & -22.3 & -45.4 \\
\hline Them & -46.3 & -51.9 & -5.2 & 38.8 & 27.2 & 21.5 & 40.5 & 19.2 & 10.4 & 10.7 & -19.1 & -45.7 \\
\hline Tirstrup & -50.7 & -45.8 & 4.0 & 38.5 & 33.2 & 16.8 & 30.0 & 14.9 & 0.6 & 25.0 & -14.3 & -52.2 \\
\hline Torkilstrup & -51.0 & -46.1 & -6.5 & 37.7 & 36.2 & 20.1 & 35.1 & 21.9 & 7.7 & 3.0 & -25.4 & -32.6 \\
\hline Ubby & -37.1 & -37.1 & 6.1 & 37.0 & 21.6 & 12.2 & 33.1 & 30.8 & -3.0 & -0.7 & -24.3 & -38.5 \\
\hline Vaegtergangen & -40.2 & -56.6 & -11.6 & 30.0 & 59.6 & 27.4 & 35.9 & 21.0 & 17.1 & 4.3 & -27.0 & -59.9 \\
\hline Vasbygade & -8.9 & -11.1 & 2.9 & 12.5 & 13.3 & 4.6 & 19.4 & 2.2 & 7.4 & 7.5 & -13.5 & -36.2 \\
\hline Vermlandsgade & -17.1 & -26.3 & -0.5 & 9.5 & 13.8 & 6.7 & 17.5 & 10.5 & 12.2 & 8.8 & -3.0 & -32.2 \\
\hline Viby & -51.8 & -44.5 & -17.7 & 38.5 & 37.7 & 40.7 & 33.1 & 19.0 & 4.5 & 6.0 & -23.0 & -42.5 \\
\hline Mean & -43.7 & -43.7 & -4.5 & 30.3 & 32.1 & 19.3 & 31.6 & 15.6 & 7.5 & 10.1 & -15.2 & -39.5 \\
\hline Standard deviation & 12.5 & 11.2 & 11.1 & 10.3 & 14.1 & 8.3 & 13.3 & 12.6 & 8.7 & 11.5 & 27.4 & 13.6 \\
\hline
\end{tabular}


Table S8: Spearman correlation analysis to determine factors influencing the total waste generation and the number of visitors at household recycling centres. Correlation analysis was based on data collected from 2010 to 2016 . .

\begin{tabular}{lcccrc}
\hline \multirow{2}{*}{ Municipalities } & \multicolumn{3}{c}{ Correlation between total mass and: } & \multicolumn{2}{c}{ Correlation between number and visitors and: } \\
& Visitors & Inhabitants & Density (inhabitant/km2) & Inhabitants & Density (inhabitant/km2) \\
\hline Dragør & 0.50 & $\mathbf{- 0 . 0 4}$ & $\mathbf{0 . 4 6}$ & $\mathbf{- 0 . 0 7}$ & 0.99 \\
Frederiksberg & 0.96 & 0.99 & 0.95 & $\mathbf{0 . 3 2}$ \\
Hvidovre & 0.75 & $\mathbf{- 0 . 3 6}$ & $\mathbf{- 0 . 4 3}$ & $\mathbf{- 0 . 3 9}$ & -0.64 \\
Copenhagen & 0.79 & -0.64 & $\mathbf{- 0 . 3 2}$ & $\mathbf{- 0 . 4 6}$ & $\mathbf{- 0 . 3 9}$ \\
Taarnby & 0.71 & $\mathbf{- 0 . 3 6}$ & $\mathbf{0 . 2 5}$ & $\mathbf{- 0 . 0 7}$ \\
Norddjurs & 0.86 & -0.75 & -0.68 & $\mathbf{0 . 1 4}$ \\
Syddjurs & 0.71 & -0.64 & $\mathbf{- 0 . 2 1}$ & $\mathbf{0 . 0 0}$ \\
\hline
\end{tabular}

${ }^{a}$ The number of recycling centres per municipalities and number of visitors are shown in Table 1

${ }^{b}: \mathrm{kg}$ wet mass waste per working day for the office area investigated.

$c$ : Number employees per working days.

${ }^{d}$ :Employees at work.

Table S9: Spearman correlation analysis to determine factors influencing the total waste generation and the number of visitors at household recycling centres. Correlation analysis was computed per year based on the data from municipalities (Table 1) Dargør, Frederiksberg, Hvidovre, Copenhagen, Taarnby, Norddjurs and Syddjurs.

\begin{tabular}{lccccc}
\hline \multirow{2}{*}{ Municipalities } & \multicolumn{3}{c}{ Correlation between total mass and: } & \multicolumn{2}{c}{ Correlation between number and visitors and: } \\
& Visitors & Inhabitants & Density (inhabitant/km2) & Inhabitants & Density (inhabitant/km2) \\
\hline 2010 & 0.82 & $\mathbf{0 . 1 8}$ & -0.21 & $\mathbf{0 . 0 7}$ & -0.32 \\
2011 & 0.93 & $\mathbf{0 . 1 4}$ & -0.32 & $\mathbf{0 . 0 7}$ & -0.32 \\
2012 & 0.93 & $\mathbf{0 . 1 4}$ & -0.32 & $\mathbf{0 . 0 7}$ & -0.32 \\
2013 & 0.79 & $\mathbf{0 . 3 2}$ & -0.21 & $\mathbf{0 . 1 1}$ & -0.36 \\
2014 & 0.93 & $\mathbf{0 . 3 6}$ & -0.14 & $\mathbf{0 . 2 9}$ & -0.14 \\
2015 & 0.93 & $\mathbf{0 . 4 3}$ & -0.14 & $\mathbf{0 . 3 2}$ & -0.14 \\
2016 & 0.96 & 0.77 & 0.70 & 0.63 & 0.67 \\
\hline
\end{tabular}

${ }^{a}$ Wet mass

${ }^{b}: k g$ wet mass waste per working day for the office area investigated.

c: Number employees per working days.

Table S10: Correlation between number of visitors and household size

\begin{tabular}{|c|c|c|c|c|c|c|c|c|}
\hline & $\begin{array}{l}\text { Visitor } \\
\mathrm{s}\end{array}$ & $\begin{array}{l}\text { area_km } \\
2\end{array}$ & $\begin{array}{l}\begin{array}{l}\text { Househol } \\
\mathrm{d}\end{array} \\
\end{array}$ & Person & $\begin{array}{l}\text { MF_Househol } \\
\text { d }\end{array}$ & $\begin{array}{l}\text { MF_Perso } \\
\text { n }\end{array}$ & $\begin{array}{l}\text { SF_Househol } \\
\text { d }\end{array}$ & $\begin{array}{l}\text { SF_Perso } \\
\text { n }\end{array}$ \\
\hline Visitors & 1 & & & & & & & \\
\hline area_km2 & 0.41 & 1 & & & & & & \\
\hline Household & 0.5 & -0.22 & 1 & & & & & \\
\hline Person & 0.51 & -0.21 & 1 & 1 & & & & \\
\hline MF_Household & 0.43 & -0.29 & 0.99 & 0.99 & 1 & & & \\
\hline MF_Person & 0.42 & -0.29 & 0.99 & 0.99 & 1 & 1 & & \\
\hline SF_Household & 0.84 & 0.58 & 0.38 & 0.4 & 0.28 & 0.28 & 1 & \\
\hline SF_Person & 0.85 & 0.47 & 0.48 & 0.5 & 0.39 & 0.39 & 0.99 & 1 \\
\hline
\end{tabular}

Table S11: Results of the test Ljunk-Box

\begin{tabular}{lccc}
\hline Areas & X-squared & Df & p-value \\
\hline Greater Copenhagen & 47.19 & 1 & $6.44 \mathrm{E}-12 * * *$ \\
\hline
\end{tabular}




\begin{tabular}{lccc}
\hline Roskilde & 35.766 & 1 & $2.23 \mathrm{E}-09^{* * *}$ \\
Djursland & 19.783 & 1 & $8.68 \mathrm{E}-06^{* * *}$ \\
Silkeborg & 37.346 & 1 & $9.89 \mathrm{E}-10^{* * *}$ \\
\hline
\end{tabular}

p-values $<0.05$ : significant autocorrelation; $* * *$ Highly significant autocorrelation

Table S12: Univariate Box-Pierce test

\begin{tabular}{|c|c|c|c|c|c|c|c|}
\hline Areas & Municipalities & Recyling centers & statistic & p.value & parameter & method & Answer \\
\hline \multirow{8}{*}{ Djursland } & Norddjurs & Glesborg & 26.71535 & 0.00000 & 1 & Box-Pierce test & significant \\
\hline & Norddjurs & Grenaa & 0.00005 & 0.99442 & 1 & Box-Pierce test & No-sign \\
\hline & Norddjurs & Roende & 24.64980 & 0.00000 & 1 & Box-Pierce test & significant \\
\hline & Norddjurs & Tirstrup & 24.68332 & 0.00000 & 1 & Box-Pierce test & significant \\
\hline & Syddjurs & Ebeltoft & 27.15883 & 0.00000 & 1 & Box-Pierce test & significant \\
\hline & Syddjurs & Hornslet & 21.93223 & 0.00000 & 1 & Box-Pierce test & significant \\
\hline & Syddjurs & Knebel & 27.57318 & 0.00000 & 1 & Box-Pierce test & significant \\
\hline & Syddjurs & Ryomgaard & 26.50164 & 0.00000 & 1 & Box-Pierce test & significant \\
\hline \multirow{4}{*}{ Silkeborg } & Silkeborg & Tandskov & 30.44238 & 0.00000 & 1 & Box-Pierce test & significant \\
\hline & Silkeborg & Tietgensvej & 36.15314 & 0.00000 & 1 & Box-Pierce test & significant \\
\hline & Silkeborg & Gjern & 24.43454 & 0.00000 & 1 & Box-Pierce test & significant \\
\hline & Silkeborg & Them & 28.12465 & 0.00000 & 1 & Box-Pierce test & significant \\
\hline \multirow{10}{*}{$\begin{array}{c}\text { Greater } \\
\text { Copenhagen }\end{array}$} & Dragoer & Dragoer & 32.61141 & 0.00000 & 1 & Box-Pierce test & significant \\
\hline & Frederiksberg & Bispeengen & 31.58424 & 0.00000 & 1 & Box-Pierce test & significant \\
\hline & Hvidovre & Hvidovre & 48.81693 & 0.00000 & 1 & Box-Pierce test & significant \\
\hline & Copenhagen & Borgervaenget & 32.19019 & 0.00000 & 1 & Box-Pierce test & significant \\
\hline & Copenhagen & Chrisiania & 10.69465 & 0.00107 & 1 & Box-Pierce test & significant \\
\hline & Copenhagen & Kulbanevej & 58.15301 & 0.00000 & 1 & Box-Pierce test & significant \\
\hline & Copenhagen & Vasbygade & 41.82745 & 0.00000 & 1 & Box-Pierce test & significant \\
\hline & Copenhagen & Vermlandsgade & 56.07806 & 0.00000 & 1 & Box-Pierce test & significant \\
\hline & Taarnby & Kirstinehoej & 43.03532 & 0.00000 & 1 & Box-Pierce test & significant \\
\hline & Taarnby & Vaegtergangen & 28.24106 & 0.00000 & 1 & Box-Pierce test & significant \\
\hline \multirow{14}{*}{ Central Zealand } & Kalundborg & Bregninge & 32.21069 & 0.00000 & 1 & Box-Pierce test & significant \\
\hline & Kalundborg & Goerlev & 32.43045 & 0.00000 & 1 & Box-Pierce test & significant \\
\hline & Kalundborg & Hoeng & 21.67075 & 0.00000 & 1 & Box-Pierce test & significant \\
\hline & Kalundborg & Kalundborg & 30.62308 & 0.00000 & 1 & Box-Pierce test & significant \\
\hline & Kalundborg & Sejeroe & 0.61653 & 0.43234 & 1 & Box-Pierce test & No-sign \\
\hline & Kalundborg & Ubby & 20.75011 & 0.00001 & 1 & Box-Pierce test & significant \\
\hline & Koege & Bjaeverskov & 31.99063 & 0.00000 & 1 & Box-Pierce test & significant \\
\hline & Koege & Koege & 27.30350 & 0.00000 & 1 & Box-Pierce test & significant \\
\hline & Lejre & Torkilstrup & 22.92409 & 0.00000 & 1 & Box-Pierce test & significant \\
\hline & Roskilde & Jyllinge & 35.42817 & 0.00000 & 1 & Box-Pierce test & significant \\
\hline & Roskilde & Roskilde & 25.64590 & 0.00000 & 1 & Box-Pierce test & significant \\
\hline & Roskilde & Viby & 31.65126 & 0.00000 & 1 & Box-Pierce test & significant \\
\hline & Stevns & Haarlev & 8.77963 & 0.00305 & 1 & Box-Pierce test & significant \\
\hline & Stevns & Store Hedding & 32.82482 & 0.00000 & 1 & Box-Pierce test & significant \\
\hline
\end{tabular}


Table S13: Univariate test of autoregression based on on aggregrade

\begin{tabular}{lccccc}
\hline Areas & Statistic & p-value & Parameter & Method & Answer \\
\hline Djursland & 19.09252 & $1.25 \mathrm{E}-05$ & 1 & Box-Pierce test & significant \\
Greater Copenhagen & 45.54366 & $1.49 \mathrm{E}-11$ & 1 & Box-Pierce test & significant \\
Central Zealand & 34.51795 & $4.22 \mathrm{E}-09$ & 1 & Box-Pierce test & significant \\
Silkeborg & 36.04304 & $1.93 \mathrm{E}-09$ & 1 & Box-Pierce test & significan \\
\hline
\end{tabular}


Table S14: Detail composition based on main fractions for each household recycling centers from 2010 to 2016 (wet mass percentage)

\begin{tabular}{|c|c|c|c|c|c|c|c|c|c|c|}
\hline Regions & Municipaliteis & HWRC & FraktionTWF2 & 2010 & 2011 & 2012 & 2013 & 2014 & 2015 & 2016 \\
\hline Greater Copenhagen & Frederiksberg & Bispeengen & Fibres & & & 2.77 & 3.1 & 3.06 & 3.4 & 3.87 \\
\hline Greater Copenhagen & Frederiksberg & Bispeengen & Plastic & & & 0.75 & 0.78 & 0.77 & 0.87 & 1 \\
\hline Greater Copenhagen & Frederiksberg & Bispeengen & Metal & & & 4.61 & 4.69 & 4.11 & 4.33 & 4.8 \\
\hline Greater Copenhagen & Frederiksberg & Bispeengen & Wood & & & 20.11 & 19.54 & 18.72 & 19.73 & 20.13 \\
\hline Greater Copenhagen & Frederiksberg & Bispeengen & Glass & & & 1.71 & 1.21 & 1.22 & 1.05 & 1.04 \\
\hline Greater Copenhagen & Frederiksberg & Bispeengen & Garden & & & 2.2 & 5.09 & 6.39 & 7.34 & 7.72 \\
\hline Greater Copenhagen & Frederiksberg & Bispeengen & Soil & & & 9.53 & 8.64 & 9.99 & 8.96 & 8.12 \\
\hline Greater Copenhagen & Frederiksberg & Bispeengen & $\mathrm{C} \& \mathrm{D}$ & & & 39.53 & 37.78 & 38.91 & 36.78 & 34.62 \\
\hline Greater Copenhagen & Frederiksberg & Bispeengen & SMC & & & 9.44 & 9.35 & 8.3 & 8.65 & 8.03 \\
\hline Greater Copenhagen & Frederiksberg & Bispeengen & Bulky & & & & 1.86 & 1.78 & 1.81 & 1.86 \\
\hline Greater Copenhagen & Frederiksberg & Bispeengen & OW & & & 9.35 & 7.96 & 6.76 & 7.08 & 8.79 \\
\hline Greater Copenhagen & Dragoer & Dragoer & Fibres & 2.78 & 2.62 & 2.7 & 3.18 & 2.7 & 2.93 & 3.11 \\
\hline Greater Copenhagen & Dragoer & Dragoer & Plastic & 0 & 0 & 0.46 & 0.64 & 0.7 & 0.65 & 0.82 \\
\hline Greater Copenhagen & Dragoer & Dragoer & Metal & 3.62 & 3.49 & 3.92 & 3.72 & 3.59 & 3.78 & 3.81 \\
\hline Greater Copenhagen & Dragoer & Dragoer & Wood & 2.07 & 7.94 & 9.77 & 10.11 & 10.04 & 10.62 & 10.82 \\
\hline Greater Copenhagen & Dragoer & Dragoer & Glass & 1.98 & 0.68 & 0.71 & 0.81 & 0.69 & 0.68 & 0.84 \\
\hline Greater Copenhagen & Dragoer & Dragoer & Garden & 25.74 & 27.02 & 25 & 25.2 & 26.11 & 24.22 & 24.19 \\
\hline Greater Copenhagen & Dragoer & Dragoer & Soil & 13.78 & 15.09 & 15.33 & 13.93 & 15.98 & 15.77 & 14.98 \\
\hline Greater Copenhagen & Dragoer & Dragoer & $\mathrm{C} \& \mathrm{D}$ & 20.07 & 23.63 & 22.42 & 23.3 & 23.16 & 24.14 & 23.21 \\
\hline Greater Copenhagen & Dragoer & Dragoer & SMC & 12.9 & 11.84 & 12.04 & 11.48 & 10.74 & 10.46 & 10.52 \\
\hline Greater Copenhagen & Dragoer & Dragoer & Bulky & 6.06 & 0.93 & 0.19 & 0.81 & 0.81 & 0.77 & 0.89 \\
\hline Greater Copenhagen & Dragoer & Dragoer & OW & 10.98 & 6.75 & 7.47 & 6.82 & 5.47 & 5.97 & 6.81 \\
\hline Greater Copenhagen & Hvidovre & Hvidovre & Fibres & 1.96 & 1.95 & 2.27 & 2.37 & 2.29 & 2.07 & 2.43 \\
\hline Greater Copenhagen & Hvidovre & Hvidovre & Plastic & 0.07 & 0.08 & 0.08 & 0.65 & 0.91 & 0.88 & 0.95 \\
\hline Greater Copenhagen & Hvidovre & Hvidovre & Metal & 4.26 & 4.02 & 3.73 & 3.73 & 3.49 & 3.37 & 3.72 \\
\hline Greater Copenhagen & Hvidovre & Hvidovre & Wood & 3.6 & 12.41 & 12 & 12.32 & 12.47 & 13.45 & 13.89 \\
\hline Greater Copenhagen & Hvidovre & Hvidovre & Glass & 1.52 & 1.59 & 1.06 & 1.08 & 1.03 & 1.16 & 1.13 \\
\hline Greater Copenhagen & Hvidovre & Hvidovre & Garden & 12.29 & 13.44 & 12.3 & 14.18 & 14.09 & 14.25 & 14.37 \\
\hline Greater Copenhagen & Hvidovre & Hvidovre & Soil & 15.51 & 14.75 & 17.44 & 16.66 & 15.91 & 15.3 & 15.62 \\
\hline
\end{tabular}




\begin{tabular}{|c|c|c|c|c|c|c|c|c|c|c|}
\hline Greater Copenhagen & Hvidovre & Hvidovre & $\mathrm{C} \& \mathrm{D}$ & 26.07 & 32.65 & 32.97 & 32.23 & 34.5 & 34.64 & 32 \\
\hline Greater Copenhagen & Hvidovre & Hvidovre & SMC & 11.95 & 10.64 & 9.54 & 8.93 & 7.76 & 6.88 & 6.74 \\
\hline Greater Copenhagen & Hvidovre & Hvidovre & Bulky & 6.74 & 1.1 & 0.23 & 0.91 & 1.07 & 0.99 & 1.08 \\
\hline Greater Copenhagen & Hvidovre & Hvidovre & OW & 16.03 & 7.37 & 8.37 & 6.93 & 6.48 & 7.03 & 8.08 \\
\hline Greater Copenhagen & Koebenhavn & Borgervaenget & Fibres & 5.83 & 5.63 & 4.15 & 3.69 & 3.86 & 3.55 & 4.01 \\
\hline Greater Copenhagen & Koebenhavn & Chrisiania & Fibres & 3.47 & & 4.92 & 0.52 & 3.12 & 3.51 & 3.41 \\
\hline Greater Copenhagen & Koebenhavn & Kulbanevej & Fibres & 3.74 & 3.15 & 2.96 & 3.06 & 2.73 & 2.62 & 2.81 \\
\hline Greater Copenhagen & Koebenhavn & Vasbygade & Fibres & 7.79 & 6.44 & 5.94 & 6.13 & 4.96 & 5.01 & 5.73 \\
\hline Greater Copenhagen & Koebenhavn & Vermlandsgade & Fibres & 4.03 & 3.33 & 3.19 & 3.41 & 3.08 & 3.06 & 3.49 \\
\hline Greater Copenhagen & Koebenhavn & Borgervaenget & Plastic & 0.17 & 0.26 & 0.53 & 0.85 & 0.95 & 1.04 & 0.92 \\
\hline Greater Copenhagen & Koebenhavn & Chrisiania & Plastic & & & & & 0 & 0.3 & 0.6 \\
\hline Greater Copenhagen & Koebenhavn & Kulbanevej & Plastic & 0.1 & 0.1 & 0.06 & 0.66 & 0.91 & 0.88 & 0.86 \\
\hline Greater Copenhagen & Koebenhavn & Vasbygade & Plastic & 0.35 & 0.28 & 0.08 & 0.6 & 1.13 & 1.27 & 1.09 \\
\hline Greater Copenhagen & Koebenhavn & Vermlandsgade & Plastic & 0.14 & 0.11 & 0.49 & 0.96 & 0.98 & 1.01 & 1.13 \\
\hline Greater Copenhagen & Koebenhavn & Borgervaenget & Metal & 4.86 & 4.89 & 4.7 & 4.37 & 4.44 & 4.26 & 5.14 \\
\hline Greater Copenhagen & Koebenhavn & Chrisiania & Metal & 8.48 & 8.1 & 7.64 & 6.15 & 5.38 & 5.11 & 4.81 \\
\hline Greater Copenhagen & Koebenhavn & Kulbanevej & Metal & 5.01 & 4.63 & 4.09 & 3.95 & 3.62 & 3.61 & 3.71 \\
\hline Greater Copenhagen & Koebenhavn & Vasbygade & Metal & 6.64 & 6.11 & 6.32 & 5.58 & 5.24 & 5.91 & 4.93 \\
\hline Greater Copenhagen & Koebenhavn & Vermlandsgade & Metal & 5.8 & 5.08 & 4.62 & 4.53 & 4.11 & 4.02 & 4.74 \\
\hline Greater Copenhagen & Koebenhavn & Borgervaenget & Wood & 17.04 & 18.68 & 19.62 & 19.91 & 20.19 & 19.96 & 19.78 \\
\hline Greater Copenhagen & Koebenhavn & Kulbanevej & Wood & 4.36 & 14.97 & 14.59 & 14.92 & 14.76 & 15.62 & 15.68 \\
\hline Greater Copenhagen & Koebenhavn & Vasbygade & Wood & 5.86 & 20.82 & 22.42 & 23.28 & 21.93 & 23.51 & 21.26 \\
\hline Greater Copenhagen & Koebenhavn & Vermlandsgade & Wood & 5.49 & 16.81 & 17.97 & 18.39 & 19.14 & 18.77 & 19.68 \\
\hline Greater Copenhagen & Koebenhavn & Borgervaenget & Glass & 1.14 & 1.28 & 1.16 & 1.39 & 1.39 & 1.66 & 1.19 \\
\hline Greater Copenhagen & Koebenhavn & Kulbanevej & Glass & 1.07 & 1.29 & 1.05 & 1.16 & 1.02 & 0.95 & 0.98 \\
\hline Greater Copenhagen & Koebenhavn & Vasbygade & Glass & 1.21 & 1.31 & 1.25 & 1.39 & 1.6 & 1.56 & 1.05 \\
\hline Greater Copenhagen & Koebenhavn & Vermlandsgade & Glass & 1.49 & 1.72 & 1.46 & 1.14 & 1.1 & 1.31 & 1.15 \\
\hline Greater Copenhagen & Koebenhavn & Borgervaenget & Garden & 7.2 & 7.16 & 5.5 & 6.05 & 6.42 & 6.19 & 7.32 \\
\hline Greater Copenhagen & Koebenhavn & Chrisiania & Garden & 0.96 & 0.37 & 0.51 & 2.23 & & & \\
\hline Greater Copenhagen & Koebenhavn & Kulbanevej & Garden & 8.87 & 9.61 & 9.22 & 10.14 & 12.18 & 10.44 & 10.72 \\
\hline Greater Copenhagen & Koebenhavn & Vasbygade & Garden & 3.87 & 3.9 & 3.22 & 3.67 & 5.04 & 3.73 & 3.16 \\
\hline Greater Copenhagen & Koebenhavn & Vermlandsgade & Garden & 5.78 & 5.88 & 5.65 & 6.44 & 6.27 & 6.57 & 7.27 \\
\hline
\end{tabular}




\begin{tabular}{|c|c|c|c|c|c|c|c|c|c|c|}
\hline Greater Copenhagen & Koebenhavn & Borgervaenget & Soil & 10.52 & 9.75 & 7.47 & 9.02 & 8.51 & 9.39 & 9.92 \\
\hline Greater Copenhagen & Koebenhavn & Chrisiania & Soil & & & & & 16.15 & 25.65 & 35.87 \\
\hline Greater Copenhagen & Koebenhavn & Kulbanevej & Soil & 10.96 & 11.13 & 13.57 & 13.4 & 12.64 & 14.31 & 15.05 \\
\hline Greater Copenhagen & Koebenhavn & Vasbygade & Soil & 5.73 & 5.09 & 5.44 & 5.44 & 6.92 & 3.98 & 4.88 \\
\hline Greater Copenhagen & Koebenhavn & Vermlandsgade & Soil & 9.9 & 11.69 & 14.48 & 11.61 & 12.92 & 15.5 & 9.88 \\
\hline Greater Copenhagen & Koebenhavn & Borgervaenget & $\mathrm{C} \& \mathrm{D}$ & 25.33 & 30.33 & 37.81 & 36.51 & 35.5 & 36.77 & 33.81 \\
\hline Greater Copenhagen & Koebenhavn & Chrisiania & $\mathrm{C} \& \mathrm{D}$ & 20.42 & 20.44 & 24.45 & 28.8 & 22.8 & 23.45 & 20.64 \\
\hline Greater Copenhagen & Koebenhavn & Kulbanevej & $\mathrm{C} \& \mathrm{D}$ & 24.24 & 33.91 & 36.7 & 35.09 & 35.69 & 36.2 & 32.68 \\
\hline Greater Copenhagen & Koebenhavn & Vasbygade & $\mathrm{C} \& \mathrm{D}$ & 19.06 & 30.95 & 33.42 & 32.53 & 32.96 & 31.35 & 30.61 \\
\hline Greater Copenhagen & Koebenhavn & Vermlandsgade & $C \& D$ & 25.07 & 34.85 & 34.22 & 35.15 & 35.33 & 33.33 & 30.75 \\
\hline Greater Copenhagen & Koebenhavn & Borgervaenget & SMC & 9.78 & 12.62 & 10.73 & 9.89 & 10.42 & 9.47 & 8.54 \\
\hline Greater Copenhagen & Koebenhavn & Chrisiania & SMC & 24.86 & 29.65 & 22.07 & 23.3 & 28.75 & 24.85 & 19.64 \\
\hline Greater Copenhagen & Koebenhavn & Kulbanevej & SMC & 14.11 & 12.04 & 9.34 & 8.94 & 8.31 & 7.45 & 7.51 \\
\hline Greater Copenhagen & Koebenhavn & Vasbygade & SMC & 20.09 & 14.73 & 11.67 & 11.2 & 10.71 & 10.96 & 10.19 \\
\hline Greater Copenhagen & Koebenhavn & Vermlandsgade & SMC & 12.72 & 10.95 & 9.54 & 9.68 & 8.93 & 8.17 & 8.73 \\
\hline Greater Copenhagen & Koebenhavn & Borgervaenget & Bulky & 2.12 & 0.88 & 0.37 & 1.38 & 1.77 & 1.58 & 1.84 \\
\hline Greater Copenhagen & Koebenhavn & Chrisiania & Bulky & 21 & 23.76 & 21.9 & 18.32 & 12.18 & 9.92 & 7.72 \\
\hline Greater Copenhagen & Koebenhavn & Kulbanevej & Bulky & 8.41 & 1.08 & 0.4 & 1.4 & 1.45 & 1.35 & 1.43 \\
\hline Greater Copenhagen & Koebenhavn & Vasbygade & Bulky & 10.33 & 2.29 & 1.09 & 2.6 & 2.42 & 2.83 & 2.82 \\
\hline Greater Copenhagen & Koebenhavn & Vermlandsgade & Bulky & 11.1 & 1.96 & 0.57 & 1.67 & 1.92 & 1.62 & 2.09 \\
\hline Greater Copenhagen & Koebenhavn & Borgervaenget & OW & 16.01 & 8.53 & 7.96 & 6.95 & 6.56 & 6.11 & 7.52 \\
\hline Greater Copenhagen & Koebenhavn & Chrisiania & OW & 20.81 & 17.68 & 18.51 & 20.68 & 11.61 & 7.21 & 7.31 \\
\hline Greater Copenhagen & Koebenhavn & Kulbanevej & OW & 19.12 & 8.09 & 8.02 & 7.28 & 6.68 & 6.56 & 8.56 \\
\hline Greater Copenhagen & Koebenhavn & Vasbygade & OW & 19.06 & 8.1 & 9.16 & 7.57 & 7.08 & 9.89 & 14.27 \\
\hline Greater Copenhagen & Koebenhavn & Vermlandsgade & OW & 18.46 & 7.61 & 7.8 & 7.02 & 6.22 & 6.64 & 11.09 \\
\hline Greater Copenhagen & Taarnby & Kirstinehoej & Fibres & 1.6 & 1.58 & 1.62 & 1.86 & 1.9 & 1.98 & 1.93 \\
\hline Greater Copenhagen & Taarnby & Vaegtergangen & Fibres & 2.85 & 3.79 & 3.23 & 3.69 & 3.54 & 3.48 & 4.08 \\
\hline Greater Copenhagen & Taarnby & Kirstinehoej & Plastic & 0.03 & 0.02 & 0.45 & 0.81 & 0.84 & 0.91 & 0.99 \\
\hline Greater Copenhagen & Taarnby & Vaegtergangen & Plastic & & & & 0.54 & 1.18 & 1.29 & 1.57 \\
\hline Greater Copenhagen & Taarnby & Kirstinehoej & Metal & 4.4 & 4.1 & 3.99 & 4.02 & 3.79 & 3.91 & 4.01 \\
\hline Greater Copenhagen & Taarnby & Vaegtergangen & Metal & 6 & 6.07 & 5.83 & 5.1 & 4.72 & 4.48 & 4.92 \\
\hline Greater Copenhagen & Taarnby & Kirstinehoej & Wood & 2.99 & 11.19 & 12.12 & 13.17 & 13.19 & 14.93 & 14.64 \\
\hline
\end{tabular}




\begin{tabular}{|c|c|c|c|c|c|c|c|c|c|c|}
\hline Greater Copenhagen & Taarnby & Vaegtergangen & Wood & 2.03 & 10.62 & 11.66 & 14.98 & 11.37 & 10.85 & 11.62 \\
\hline Greater Copenhagen & Taarnby & Kirstinehoej & Glass & 1.27 & 1.28 & 1.21 & 1.03 & 0.92 & 1.04 & 1.02 \\
\hline Greater Copenhagen & Taarnby & Vaegtergangen & Glass & 2.95 & 0.11 & & & & & \\
\hline Greater Copenhagen & Taarnby & Kirstinehoej & Garden & 16.43 & 17.9 & 16.15 & 16.42 & 16.85 & 15.9 & 16.24 \\
\hline Greater Copenhagen & Taarnby & Vaegtergangen & Garden & 23.78 & 27.63 & 24.57 & 22.58 & 28.43 & 35.02 & 34.24 \\
\hline Greater Copenhagen & Taarnby & Kirstinehoej & Soil & 19.27 & 18.95 & 18.72 & 17.6 & 19.09 & 17.37 & 17.3 \\
\hline Greater Copenhagen & Taarnby & Vaegtergangen & Soil & 12.8 & 6.28 & 10.42 & 12.49 & 9.87 & 12.24 & 10.16 \\
\hline Greater Copenhagen & Taarnby & Kirstinehoej & $\mathrm{C} \& \mathrm{D}$ & 24.18 & 28.51 & 29.86 & 29.34 & 28.38 & 29.35 & 29.04 \\
\hline Greater Copenhagen & Taarnby & Vaegtergangen & $\mathrm{C} \& \mathrm{D}$ & 12.8 & 13.65 & 14.64 & 16.07 & 18.78 & 12.74 & 12.15 \\
\hline Greater Copenhagen & Taarnby & Kirstinehoej & SMC & 10.06 & 9.05 & 8.25 & 8.27 & 7.94 & 7.36 & 6.87 \\
\hline Greater Copenhagen & Taarnby & Vaegtergangen & SMC & 17.58 & 18.2 & 15.76 & 13.57 & 11.16 & 10.65 & 9.84 \\
\hline Greater Copenhagen & Taarnby & Kirstinehoej & Bulky & 7.55 & 0.61 & 0.24 & 0.85 & 1.01 & 0.99 & 0.96 \\
\hline Greater Copenhagen & Taarnby & Vaegtergangen & Bulky & 5.89 & 0 & & 1.74 & 1.5 & 1.19 & 1.68 \\
\hline Greater Copenhagen & Taarnby & Kirstinehoej & OW & 12.23 & 6.79 & 7.38 & 6.64 & 6.1 & 6.26 & 7 \\
\hline Greater Copenhagen & Taarnby & Vaegtergangen & OW & 13.31 & 13.65 & 13.9 & 9.23 & 9.44 & 8.06 & 9.74 \\
\hline Central Zealand & Koege & Bjaeverskov & Fibres & 1.65 & 1.45 & 2.01 & 1.7 & 1.66 & 1.58 & 1.29 \\
\hline Central Zealand & Koege & Koege & Fibres & 1.43 & 1.24 & 1.22 & 1.22 & 1.26 & 1.24 & 1.15 \\
\hline Central Zealand & Koege & Bjaeverskov & Plastic & 0 & 0.06 & 0 & 0 & 0.01 & 0.03 & 0.02 \\
\hline Central Zealand & Koege & Koege & Plastic & 0.02 & 0.01 & 0.01 & 0.04 & 0.06 & 0.08 & 0.09 \\
\hline Central Zealand & Koege & Bjaeverskov & Metal & 5.01 & 4.89 & 5.27 & 4.52 & 5.15 & 4.72 & 4.11 \\
\hline Central Zealand & Koege & Koege & Metal & 4 & 3.93 & 4.07 & 3.07 & 3.94 & 4.09 & 4.35 \\
\hline Central Zealand & Koege & Bjaeverskov & Wood & 0 & & & 0 & 0 & 1.37 & 2.2 \\
\hline Central Zealand & Koege & Koege & Wood & 0 & & & 0 & 0 & 0.98 & 4.87 \\
\hline Central Zealand & Koege & Bjaeverskov & Glass & 0.73 & 0.96 & 1.89 & 2.11 & 1.74 & 1.3 & 2.23 \\
\hline Central Zealand & Koege & Koege & Glass & 1.46 & 1.54 & 1.73 & 1.22 & 1.67 & 1.74 & 1.56 \\
\hline Central Zealand & Koege & Bjaeverskov & Garden & 29.3 & 28.25 & 26.6 & 26.79 & 31.5 & 31.46 & 27.78 \\
\hline Central Zealand & Koege & Koege & Garden & 27.81 & 23.27 & 21.9 & 22.27 & 23.19 & 23.4 & 24.58 \\
\hline Central Zealand & Koege & Bjaeverskov & Soil & 9.33 & 12.54 & 10.78 & 7.93 & 10.08 & 10.9 & 11.67 \\
\hline Central Zealand & Koege & Koege & Soil & 9.64 & 8.43 & 8.18 & 8.15 & 9.1 & 9.07 & 8.65 \\
\hline Central Zealand & Koege & Bjaeverskov & $\mathrm{C} \& \mathrm{D}$ & 21.34 & 17.74 & 19.34 & 25.02 & 22.24 & 21.78 & 25.12 \\
\hline Central Zealand & Koege & Koege & $C \& D$ & 24.62 & 30 & 29.98 & 30.17 & 29.28 & 28.43 & 28.94 \\
\hline Central Zealand & Koege & Bjaeverskov & SMC & 9.87 & 13.67 & 11.25 & 10.43 & 10.31 & 14.62 & 11.36 \\
\hline
\end{tabular}




\begin{tabular}{|c|c|c|c|c|c|c|c|c|c|c|}
\hline Central Zealand & Koege & Koege & SMC & 9.4 & 11.38 & 9.52 & 9.26 & 10.5 & 13.21 & 7.41 \\
\hline Central Zealand & Koege & Bjaeverskov & Bulky & 12.95 & 10.55 & 13.73 & 11.7 & 11.23 & 6.56 & 9.09 \\
\hline Central Zealand & Koege & Koege & Bulky & 13.41 & 11.4 & 15.97 & 15.05 & 13.49 & 11.94 & 12.37 \\
\hline Central Zealand & Koege & Bjaeverskov & OW & 9.82 & 9.89 & 9.14 & 9.78 & 6.07 & 5.69 & 5.14 \\
\hline Central Zealand & Koege & Koege & OW & 8.21 & 8.79 & 7.41 & 9.54 & 7.51 & 5.83 & 6.03 \\
\hline Central Zealand & Kalundborg & Bregninge & Fibres & 2.73 & 2.55 & 2.91 & 2.61 & 2.41 & 2.49 & 2.59 \\
\hline Central Zealand & Kalundborg & Goerlev & Fibres & 4.27 & 4.03 & 4.02 & 3.73 & 3.26 & 3.28 & 2.94 \\
\hline Central Zealand & Kalundborg & Hoeng & Fibres & 5.53 & 5.64 & 5.93 & 5.06 & 4.34 & 3.65 & 3.64 \\
\hline Central Zealand & Kalundborg & Kalundborg & Fibres & 4.41 & 4.47 & 4.44 & 4.67 & 3.8 & 3.45 & 3.51 \\
\hline Central Zealand & Kalundborg & Sejeroe & Fibres & 3.52 & 7.59 & 4.31 & 2.36 & 2.04 & 1.67 & 7.81 \\
\hline Central Zealand & Kalundborg & Ubby & Fibres & 4.54 & 4.44 & 4.29 & 5.26 & 3.36 & 3.31 & 3.84 \\
\hline Central Zealand & Kalundborg & Bregninge & Plastic & 0.09 & 0.03 & 0.04 & 0.12 & 0.15 & 0.18 & 0.15 \\
\hline Central Zealand & Kalundborg & Goerlev & Plastic & 0.11 & 0.13 & 0.12 & 0.29 & 0.14 & 0.16 & 0.19 \\
\hline Central Zealand & Kalundborg & Hoeng & Plastic & 0.13 & 0.08 & 0.19 & 0.19 & 0.23 & 0.23 & 0.24 \\
\hline Central Zealand & Kalundborg & Kalundborg & Plastic & 0 & 0.01 & 0.01 & 0.05 & 0.07 & 0.11 & 0.09 \\
\hline Central Zealand & Kalundborg & Sejeroe & Plastic & 0 & 0 & 0 & 0 & 0 & 0 & 0 \\
\hline Central Zealand & Kalundborg & Ubby & Plastic & 0.07 & 0.12 & 0.17 & 0.31 & 0.28 & 0.17 & 0.16 \\
\hline Central Zealand & Kalundborg & Bregninge & Metal & 4.89 & 5.24 & 4.71 & 8.46 & 4.15 & 4.88 & 4.95 \\
\hline Central Zealand & Kalundborg & Goerlev & Metal & 4.53 & 4.73 & 4.69 & 3.16 & 4.27 & 4.57 & 4.99 \\
\hline Central Zealand & Kalundborg & Hoeng & Metal & 5.01 & 4.93 & 4.85 & 3.38 & 4.18 & 4.64 & 4.74 \\
\hline Central Zealand & Kalundborg & Kalundborg & Metal & 3.19 & 4.25 & 3.87 & 4.13 & 4.74 & 3.87 & 5.22 \\
\hline Central Zealand & Kalundborg & Sejeroe & Metal & 7.48 & 9.17 & 4.05 & 10.46 & 8.31 & 9.16 & 10.84 \\
\hline Central Zealand & Kalundborg & Ubby & Metal & 4.63 & 5.72 & 4.78 & 4.01 & 4.63 & 3.77 & 5.1 \\
\hline Central Zealand & Kalundborg & Bregninge & Wood & 0 & & & 0 & 0 & 2.58 & 10.91 \\
\hline Central Zealand & Kalundborg & Goerlev & Wood & 0 & & & 0 & 0 & 2.18 & 6.05 \\
\hline Central Zealand & Kalundborg & Hoeng & Wood & 0 & & & 0 & 0 & 2.06 & 8.31 \\
\hline Central Zealand & Kalundborg & Kalundborg & Wood & 0 & & & 0 & 0 & 2.36 & 9.44 \\
\hline Central Zealand & Kalundborg & Sejeroe & Wood & 0 & & & 0 & 0 & 0 & 0 \\
\hline Central Zealand & Kalundborg & Ubby & Wood & 0 & & & 0 & 0 & 2.39 & 9.02 \\
\hline Central Zealand & Kalundborg & Bregninge & Glass & 1.19 & 1.12 & 1.16 & 1.27 & 2.38 & 2.75 & 2.01 \\
\hline Central Zealand & Kalundborg & Goerlev & Glass & 1.22 & 1.54 & 1.55 & 1.61 & 2.47 & 2.96 & 2.05 \\
\hline Central Zealand & Kalundborg & Hoeng & Glass & 0.79 & 1.21 & 0.92 & 0.64 & 2.28 & 2.84 & 2.56 \\
\hline
\end{tabular}




\begin{tabular}{|c|c|c|c|c|c|c|c|c|c|c|}
\hline Central Zealand & Kalundborg & Kalundborg & Glass & 6.01 & 6.35 & 8.38 & 5.34 & 2.75 & 2.76 & 2.39 \\
\hline Central Zealand & Kalundborg & Sejeroe & Glass & 1.93 & 1.2 & 0.82 & 1.05 & 2.29 & 1.6 & 1.55 \\
\hline Central Zealand & Kalundborg & Ubby & Glass & 0.93 & 0.77 & 0.8 & 0.7 & 3.42 & 2.67 & 2.43 \\
\hline Central Zealand & Kalundborg & Bregninge & Garden & 29.68 & 27.45 & 29.05 & 32.17 & 31.48 & 29.36 & 28.3 \\
\hline Central Zealand & Kalundborg & Goerlev & Garden & 28.35 & 30.29 & 30.23 & 32.67 & 33.24 & 32.76 & 28.97 \\
\hline Central Zealand & Kalundborg & Hoeng & Garden & 24.97 & 25.96 & 26.97 & 27.26 & 26.68 & 27.08 & 26.79 \\
\hline Central Zealand & Kalundborg & Kalundborg & Garden & 23.38 & 22.97 & 25.47 & 28.89 & 27.15 & 25.64 & 27.03 \\
\hline Central Zealand & Kalundborg & Sejeroe & Garden & 27.96 & 0 & 54.92 & 0 & 0 & 0 & 0 \\
\hline Central Zealand & Kalundborg & Ubby & Garden & 24.41 & 22.96 & 27.63 & 29.7 & 22.9 & 28.34 & 26.03 \\
\hline Central Zealand & Kalundborg & Bregninge & Soil & 2.94 & 6.61 & 6.41 & 7.1 & 7 & 6.59 & 6.95 \\
\hline Central Zealand & Kalundborg & Goerlev & Soil & 3.77 & 4.94 & 5.78 & 5.65 & 5.02 & 5 & 4.76 \\
\hline Central Zealand & Kalundborg & Hoeng & Soil & 4.43 & 8.09 & 7.1 & 5.73 & 9.09 & 7.77 & 7.56 \\
\hline Central Zealand & Kalundborg & Kalundborg & Soil & 9.6 & 7.26 & 6.19 & 7.91 & 6.37 & 5.36 & 6.25 \\
\hline Central Zealand & Kalundborg & Sejeroe & Soil & 5.44 & 0 & 0 & 0 & 0 & 0 & 0 \\
\hline Central Zealand & Kalundborg & Ubby & Soil & 5.43 & 6.26 & 7.73 & 8.21 & 9.12 & 5.25 & 6.87 \\
\hline Central Zealand & Kalundborg & Bregninge & $C \& D$ & 22.64 & 21.58 & 21.08 & 10.07 & 18.26 & 19.67 & 20.28 \\
\hline Central Zealand & Kalundborg & Goerlev & C\&D & 21.02 & 20.23 & 19.32 & 19.2 & 19.59 & 18.67 & 24.26 \\
\hline Central Zealand & Kalundborg & Hoeng & $\mathrm{C} \& \mathrm{D}$ & 25.26 & 21.01 & 19.52 & 22.85 & 20.37 & 21.21 & 20.27 \\
\hline Central Zealand & Kalundborg & Kalundborg & $C \& D$ & 23.65 & 22.55 & 20.8 & 13.19 & 24.19 & 29.51 & 23.1 \\
\hline Central Zealand & Kalundborg & Sejeroe & $\mathrm{C} \& \mathrm{D}$ & 20.43 & 28.47 & 10.4 & 26.71 & 38.66 & 30.27 & 28.04 \\
\hline Central Zealand & Kalundborg & Ubby & C\&D & 26.22 & 24.53 & 18.79 & 13.9 & 25.18 & 24.18 & 23.44 \\
\hline Central Zealand & Kalundborg & Bregninge & SMC & 15.03 & 13.38 & 14.39 & 14.66 & 18.46 & 14.5 & 13.32 \\
\hline Central Zealand & Kalundborg & Goerlev & SMC & 13.01 & 12.92 & 15.6 & 14.35 & 13.64 & 13.61 & 8.66 \\
\hline Central Zealand & Kalundborg & Hoeng & SMC & 12.63 & 11.73 & 14.11 & 14.7 & 15.47 & 15.09 & 11.32 \\
\hline Central Zealand & Kalundborg & Kalundborg & SMC & 14.62 & 21.28 & 11.99 & 14.89 & 16.54 & 12.75 & 11.9 \\
\hline Central Zealand & Kalundborg & Sejeroe & SMC & 21.1 & 42.27 & 18.49 & 42.18 & 29.77 & 40.62 & 42.5 \\
\hline Central Zealand & Kalundborg & Ubby & SMC & 13.6 & 15.77 & 16.32 & 16.08 & 13.25 & 14.1 & 10.02 \\
\hline Central Zealand & Kalundborg & Bregninge & Bulky & 11.5 & 12.02 & 11.57 & 12.85 & 7.44 & 9.73 & 2.81 \\
\hline Central Zealand & Kalundborg & Goerlev & Bulky & 9.57 & 9.41 & 8.76 & 8.25 & 9.79 & 8.01 & 9.12 \\
\hline Central Zealand & Kalundborg & Hoeng & Bulky & 9.76 & 11.05 & 11.75 & 9.11 & 9.7 & 7.74 & 7.86 \\
\hline Central Zealand & Kalundborg & Kalundborg & Bulky & 7.9 & 2.4 & 12.13 & 12.46 & 8.6 & 9.01 & 5.28 \\
\hline Central Zealand & Kalundborg & Sejeroe & Bulky & 1.15 & 0 & 0.47 & 0.77 & 3.87 & 8.03 & 3.93 \\
\hline
\end{tabular}




\begin{tabular}{|c|c|c|c|c|c|c|c|c|c|c|}
\hline Central Zealand & Kalundborg & Ubby & Bulky & 9.76 & 8.15 & 9.23 & 9.02 & 10.34 & 7.91 & 5.16 \\
\hline Central Zealand & Kalundborg & Bregninge & OW & 9.32 & 10.02 & 8.67 & 10.68 & 8.29 & 7.28 & 7.73 \\
\hline Central Zealand & Kalundborg & Goerlev & OW & 14.13 & 11.78 & 9.93 & 11.08 & 8.58 & 8.79 & 8.02 \\
\hline Central Zealand & Kalundborg & Hoeng & OW & 11.49 & 10.31 & 8.66 & 11.07 & 7.65 & 7.69 & 6.71 \\
\hline Central Zealand & Kalundborg & Kalundborg & OW & 7.24 & 8.46 & 6.73 & 8.48 & 5.78 & 5.19 & 5.78 \\
\hline Central Zealand & Kalundborg & Sejeroe & OW & 10.99 & 11.29 & 6.53 & 16.46 & 15.06 & 8.65 & 5.34 \\
\hline Central Zealand & Kalundborg & Ubby & OW & 10.4 & 11.27 & 10.25 & 12.82 & 7.51 & 7.9 & 7.92 \\
\hline Central Zealand & Lejre & Torkilstrup & Fibres & 1.51 & 1.35 & 1.46 & 1.26 & 1.35 & 1.42 & 1.64 \\
\hline Central Zealand & Lejre & Torkilstrup & Plastic & 0.07 & 0.1 & 0.1 & 0.17 & 0.13 & 0.19 & 0.14 \\
\hline Central Zealand & Lejre & Torkilstrup & Metal & 4.94 & 5.18 & 5.17 & 4.17 & 4.29 & 4.6 & 5.2 \\
\hline Central Zealand & Lejre & Torkilstrup & Wood & 0.1 & & & 0.03 & 0 & 3.12 & 7.15 \\
\hline Central Zealand & Lejre & Torkilstrup & Glass & 1.64 & 2.45 & 2.47 & 2.26 & 2.63 & 2.68 & 2.23 \\
\hline Central Zealand & Lejre & Torkilstrup & Garden & 25.44 & 26.32 & 27.56 & 25.12 & 28.52 & 30.15 & 30.08 \\
\hline Central Zealand & Lejre & Torkilstrup & Soil & 10.39 & 9.52 & 7.7 & 7.14 & 7.63 & 9.34 & 8.72 \\
\hline Central Zealand & Lejre & Torkilstrup & $\mathrm{C} \& \mathrm{D}$ & 20.7 & 18.96 & 20.68 & 25 & 21.02 & 16.94 & 19.5 \\
\hline Central Zealand & Lejre & Torkilstrup & SMC & 13.01 & 17.35 & 12.42 & 12.31 & 12.58 & 14.92 & 13.13 \\
\hline Central Zealand & Lejre & Torkilstrup & Bulky & 12.3 & 7.4 & 12.97 & 12.22 & 14.28 & 9.74 & 5.01 \\
\hline Central Zealand & Lejre & Torkilstrup & OW & 9.91 & 11.37 & 9.48 & 10.33 & 7.56 & 6.93 & 7.21 \\
\hline Central Zealand & Stevns & Haarlev & Fibres & 1.02 & 0.9 & 0.84 & 0.89 & 1.55 & 1.53 & 1.46 \\
\hline Central Zealand & Stevns & Store_Hedding & Fibres & 1.9 & 1.68 & 1.79 & 1.65 & 1.66 & 1.71 & 1.8 \\
\hline Central Zealand & Stevns & Haarlev & Plastic & 0 & 0 & 0 & 0 & 0 & 0.05 & 0.38 \\
\hline Central Zealand & Stevns & Store_Hedding & Plastic & 0 & 0 & 0.03 & 0.04 & 0.08 & 0.11 & 0.14 \\
\hline Central Zealand & Stevns & Haarlev & Metal & 3.55 & 4.3 & 3.9 & 3.24 & 5.72 & 6.9 & 6.57 \\
\hline Central Zealand & Stevns & Store_Hedding & Metal & 4.91 & 4.59 & 6.23 & 4.13 & 5.17 & 5.63 & 5.43 \\
\hline Central Zealand & Stevns & Haarlev & Wood & 0 & & & 0 & 0 & 3.4 & 3.74 \\
\hline Central Zealand & Stevns & Store_Hedding & Wood & 0 & & & 0 & 0 & 2.9 & 5.52 \\
\hline Central Zealand & Stevns & Haarlev & Glass & 1.82 & 2.35 & 2.22 & 3.04 & 2.91 & 3.22 & 2.13 \\
\hline Central Zealand & Stevns & Store_Hedding & Glass & 2.18 & 2.34 & 2.49 & 3.06 & 2.98 & 3.08 & 2.18 \\
\hline Central Zealand & Stevns & Haarlev & Garden & 42.93 & 42.31 & 43.92 & 31.19 & 27.41 & 27.08 & 28.54 \\
\hline Central Zealand & Stevns & Store_Hedding & Garden & 24.55 & 26.92 & 24.41 & 27.19 & 29.48 & 27.58 & 27.34 \\
\hline Central Zealand & Stevns & Haarlev & Soil & 5.46 & 5.26 & 4.91 & 3.67 & 4.27 & 7.6 & 5.86 \\
\hline Central Zealand & Stevns & Store_Hedding & Soil & 8.55 & 9.73 & 9.16 & 6.96 & 7.16 & 8.12 & 8.2 \\
\hline
\end{tabular}




\begin{tabular}{|c|c|c|c|c|c|c|c|c|c|c|}
\hline Central Zealand & Stevns & Haarlev & $C \& D$ & 17.2 & 15.97 & 20.17 & 33.64 & 20.55 & 15.32 & 20.49 \\
\hline Central Zealand & Stevns & Store_Hedding & $C \& D$ & 21.98 & 20.39 & 20.22 & 20.77 & 21.03 & 19.79 & 20.59 \\
\hline Central Zealand & Stevns & Haarlev & SMC & 8.5 & 11.03 & 8.24 & 8.33 & 16.38 & 15.91 & 11.69 \\
\hline Central Zealand & Stevns & Store_Hedding & SMC & 13.15 & 13.5 & 11.38 & 13.16 & 19.1 & 19.7 & 12.51 \\
\hline Central Zealand & Stevns & Haarlev & Bulky & 10.93 & 8.78 & 8.74 & 8.16 & 12.69 & 9.5 & 10.57 \\
\hline Central Zealand & Stevns & Store_Hedding & Bulky & 12.06 & 9.06 & 13.15 & 10.82 & 5.24 & 4.44 & 7.99 \\
\hline Central Zealand & Stevns & Haarlev & OW & 8.61 & 9.1 & 7.07 & 7.82 & 8.53 & 9.49 & 8.58 \\
\hline Central Zealand & Stevns & Store_Hedding & OW & 10.72 & 11.79 & 11.14 & 12.22 & 8.1 & 6.94 & 8.31 \\
\hline Central Zealand & Roskilde & Jyllinge & Fibres & 1.62 & 1.54 & 1.54 & 1.54 & 1.4 & 1.23 & 1.37 \\
\hline Central Zealand & Roskilde & Roskilde & Fibres & 1.61 & 1.22 & 1.42 & 1.6 & 1.37 & 1.51 & 1.44 \\
\hline Central Zealand & Roskilde & Viby & Fibres & 1.6 & 1.38 & 1.35 & 1.45 & 1.28 & 1.37 & 1.33 \\
\hline Central Zealand & Roskilde & Jyllinge & Plastic & 0.19 & 0.2 & 0.17 & 0.11 & 0.08 & 0.11 & 0.14 \\
\hline Central Zealand & Roskilde & Roskilde & Plastic & 0 & 0 & 0 & 0.01 & 0.05 & 0.09 & 0.12 \\
\hline Central Zealand & Roskilde & Viby & Plastic & 0 & 0 & 0.01 & 0.06 & 0.06 & 0.05 & 0.07 \\
\hline Central Zealand & Roskilde & Jyllinge & Metal & 3.99 & 4.69 & 4.95 & 2.96 & 4.05 & 3.81 & 4.6 \\
\hline Central Zealand & Roskilde & Roskilde & Metal & 3.57 & 3.26 & 3.51 & 3.52 & 3.84 & 3.97 & 4.12 \\
\hline Central Zealand & Roskilde & Viby & Metal & 4.06 & 4.97 & 4.64 & 4.64 & 4.46 & 4.43 & 4.51 \\
\hline Central Zealand & Roskilde & Jyllinge & Wood & 0 & & & 0 & 0 & 2.39 & 5.72 \\
\hline Central Zealand & Roskilde & Roskilde & Wood & 0.32 & & & 0 & 0 & 2.84 & 8.04 \\
\hline Central Zealand & Roskilde & Viby & Wood & 0.11 & & & 0 & 0 & 2.5 & 4.15 \\
\hline Central Zealand & Roskilde & Jyllinge & Glass & 1.81 & 1.78 & 1.76 & 1.75 & 1.9 & 1.76 & 1.97 \\
\hline Central Zealand & Roskilde & Roskilde & Glass & 1.96 & 1.49 & 2.22 & 2.12 & 2.45 & 2.54 & 1.89 \\
\hline Central Zealand & Roskilde & Viby & Glass & 1.68 & 2.57 & 2.49 & 2.63 & 2.27 & 2.44 & 2.16 \\
\hline Central Zealand & Roskilde & Jyllinge & Garden & 30.89 & 30.2 & 29.43 & 27.25 & 27.45 & 25.91 & 29.01 \\
\hline Central Zealand & Roskilde & Roskilde & Garden & 20.06 & 17.17 & 20.31 & 19.7 & 20.1 & 20.34 & 21.66 \\
\hline Central Zealand & Roskilde & Viby & Garden & 25.58 & 24.22 & 25.83 & 26 & 29.29 & 26.89 & 25.07 \\
\hline Central Zealand & Roskilde & Jyllinge & Soil & 10.04 & 9.82 & 10.01 & 8.24 & 9.14 & 8.86 & 7.84 \\
\hline Central Zealand & Roskilde & Roskilde & Soil & 10.08 & 6.79 & 9.05 & 8.22 & 9.88 & 9.44 & 8.22 \\
\hline Central Zealand & Roskilde & Viby & Soil & 9.01 & 7.63 & 8.14 & 5.73 & 7.68 & 6.91 & 9.92 \\
\hline Central Zealand & Roskilde & Jyllinge & C\&D & 22.61 & 21.81 & 23.79 & 24.52 & 24.83 & 31.44 & 24.92 \\
\hline Central Zealand & Roskilde & Roskilde & $C \& D$ & 29.22 & 26.59 & 27.16 & 25.12 & 29.25 & 29.27 & 30.46 \\
\hline Central Zealand & Roskilde & Viby & $\mathrm{C} \& \mathrm{D}$ & 23.8 & 24.17 & 25.74 & 28.07 & 24.42 & 27.79 & 24.37 \\
\hline
\end{tabular}




\begin{tabular}{|c|c|c|c|c|c|c|c|c|c|c|}
\hline Central Zealand & Roskilde & Jyllinge & SMC & 10 & 13.17 & 9.66 & 10 & 10.81 & 10.71 & 9.72 \\
\hline Central Zealand & Roskilde & Roskilde & SMC & 12.43 & 28.77 & 15.33 & 15.25 & 14.22 & 15.32 & 13.4 \\
\hline Central Zealand & Roskilde & Viby & SMC & 10.66 & 14.59 & 10.9 & 10.71 & 10.83 & 12.92 & 9.77 \\
\hline Central Zealand & Roskilde & Jyllinge & Bulky & 11.35 & 8.08 & 11.31 & 13.76 & 14.36 & 8.57 & 7.57 \\
\hline Central Zealand & Roskilde & Roskilde & Bulky & 13.58 & 7.64 & 13.98 & 16.42 & 13.4 & 10.01 & 5.87 \\
\hline Central Zealand & Roskilde & Viby & Bulky & 13.45 & 9.62 & 12.35 & 11.84 & 13.58 & 9.15 & 11.89 \\
\hline Central Zealand & Roskilde & Jyllinge & OW & 7.5 & 8.71 & 7.38 & 9.86 & 5.99 & 5.19 & 7.15 \\
\hline Central Zealand & Roskilde & Roskilde & OW & 7.17 & 7.08 & 7.01 & 8.05 & 5.44 & 4.68 & 4.79 \\
\hline Central Zealand & Roskilde & Viby & OW & 10.05 & 10.86 & 8.56 & 8.87 & 6.12 & 5.55 & 6.76 \\
\hline Djursland & Syddjurs & Ebeltoft & Fibres & 3.3 & 2.9 & 3.17 & 3.18 & 2.87 & 3.16 & 3.32 \\
\hline Djursland & Syddjurs & Hornslet & Fibres & 1.81 & 1.84 & 1.71 & 1.99 & 1.67 & 1.8 & 2.19 \\
\hline Djursland & Syddjurs & Knebel & Fibres & 2.3 & 2.73 & 2.4 & 2.2 & 2.15 & 2.15 & 2.74 \\
\hline Djursland & Syddjurs & Ryomgaard & Fibres & 1.43 & 1.41 & 1.54 & 1.74 & 1.57 & 1.68 & 2.12 \\
\hline Djursland & Syddjurs & Ebeltoft & Plastic & 0.8 & 0.92 & 0.99 & 0.98 & 0.87 & 0.95 & 0.99 \\
\hline Djursland & Syddjurs & Hornslet & Plastic & 0.58 & 1.03 & 1.16 & 1.12 & 1.13 & 1.12 & 1.08 \\
\hline Djursland & Syddjurs & Knebel & Plastic & 0.72 & 0.57 & 0.85 & 1 & 1.04 & 1.09 & 1.1 \\
\hline Djursland & Syddjurs & Ryomgaard & Plastic & 0.76 & 0.98 & 0.97 & 0.97 & 0.84 & 1.06 & 1.02 \\
\hline Djursland & Syddjurs & Ebeltoft & Metal & 4.12 & 4.61 & 4.6 & 4.58 & 4.52 & 4.85 & 4.64 \\
\hline Djursland & Syddjurs & Hornslet & Metal & 4.49 & 4.84 & 4.77 & 4.92 & 4.89 & 5.42 & 4.88 \\
\hline Djursland & Syddjurs & Knebel & Metal & 4.81 & 5.01 & 5.17 & 5.34 & 5.39 & 5.4 & 5.34 \\
\hline Djursland & Syddjurs & Ryomgaard & Metal & 4.92 & 5.29 & 5.36 & 5.73 & 5.48 & 5.78 & 5.75 \\
\hline Djursland & Syddjurs & Ebeltoft & Wood & 7.07 & 6.62 & 6.96 & 7.11 & 7.12 & 7.71 & 8.23 \\
\hline Djursland & Syddjurs & Hornslet & Wood & 6.89 & 7.04 & 7.48 & 7.67 & 7.75 & 8.45 & 8.64 \\
\hline Djursland & Syddjurs & Knebel & Wood & 7.4 & 7.18 & 7.49 & 7.54 & 7.04 & 7.52 & 8.91 \\
\hline Djursland & Syddjurs & Ryomgaard & Wood & 7.12 & 6.73 & 7.66 & 7.81 & 8.05 & 8.96 & 9.44 \\
\hline Djursland & Syddjurs & Ebeltoft & Glass & 1.01 & 1.62 & 1.85 & 1.85 & 1.8 & 1.74 & 2.36 \\
\hline Djursland & Syddjurs & Hornslet & Glass & 0.67 & 1.46 & 1.65 & 1.77 & 1.76 & 2.13 & 2.22 \\
\hline Djursland & Syddjurs & Knebel & Glass & 1.07 & 1.81 & 2.28 & 2.15 & 1.94 & 2.37 & 2.41 \\
\hline Djursland & Syddjurs & Ryomgaard & Glass & 0.75 & 1.32 & 1.66 & 1.92 & 1.8 & 2.12 & 2.37 \\
\hline Djursland & Syddjurs & Ebeltoft & Garden & 34.97 & 36.14 & 38.17 & 39 & 39.86 & 39.31 & 38.92 \\
\hline Djursland & Syddjurs & Hornslet & Garden & 26.95 & 28.69 & 29.93 & 28 & 29.41 & 30.47 & 30.14 \\
\hline Djursland & Syddjurs & Knebel & Garden & 25.87 & 27.19 & 30.82 & 29.99 & 31.2 & 31.13 & 30.58 \\
\hline
\end{tabular}




\begin{tabular}{|c|c|c|c|c|c|c|c|c|c|c|}
\hline Djursland & Syddjurs & Ryomgaard & Garden & 27.43 & 26.83 & 28.93 & 26.7 & 31.19 & 29.43 & 29.33 \\
\hline Djursland & Syddjurs & Ebeltoft & Soil & 6.57 & 4.9 & 3.56 & 2.82 & 3.9 & 3.81 & 2.89 \\
\hline Djursland & Syddjurs & Hornslet & Soil & 14.61 & 9.59 & 9.21 & 9.29 & 9.51 & 7.81 & 9.17 \\
\hline Djursland & Syddjurs & Knebel & Soil & 4.86 & 2.82 & 2.83 & 2.68 & 2.43 & 2.29 & 2.29 \\
\hline Djursland & Syddjurs & Ryomgaard & Soil & 8.01 & 5.36 & 6.59 & 4.65 & 5.05 & 4.43 & 5.4 \\
\hline Djursland & Syddjurs & Ebeltoft & $\mathrm{C} \& \mathrm{D}$ & 12.4 & 13.51 & 12.57 & 12.2 & 12.28 & 11.83 & 11.98 \\
\hline Djursland & Syddjurs & Hornslet & $C \& D$ & 14.55 & 15.9 & 15.43 & 15.65 & 14.95 & 14.62 & 14.66 \\
\hline Djursland & Syddjurs & Knebel & C\&D & 16.41 & 18.38 & 15.21 & 14.86 & 17.35 & 14.96 & 13.52 \\
\hline Djursland & Syddjurs & Ryomgaard & $\mathrm{C} \& \mathrm{D}$ & 14.6 & 17.67 & 15.44 & 16.81 & 14.13 & 15.03 & 14.09 \\
\hline Djursland & Syddjurs & Ebeltoft & SMC & 5.55 & 5.75 & 6.02 & 6.31 & 5.81 & 5.76 & 5.28 \\
\hline Djursland & Syddjurs & Hornslet & SMC & 6.65 & 6.81 & 6.91 & 6.9 & 6.49 & 6.31 & 5.54 \\
\hline Djursland & Syddjurs & Knebel & SMC & 6.21 & 6.28 & 6.11 & 5.57 & 5.79 & 5.6 & 6.41 \\
\hline Djursland & Syddjurs & Ryomgaard & SMC & 6.16 & 6.5 & 6.59 & 7.23 & 6.8 & 6.59 & 6.44 \\
\hline Djursland & Syddjurs & Ebeltoft & Bulky & 3.4 & 3.66 & 3.41 & 3.75 & 3.57 & 3.27 & 3.17 \\
\hline Djursland & Syddjurs & Hornslet & Bulky & 2.71 & 2.76 & 2.85 & 2.93 & 2.72 & 2.61 & 2.88 \\
\hline Djursland & Syddjurs & Knebel & Bulky & 4.68 & 3.85 & 3.75 & 4.35 & 3.55 & 3.59 & 3.74 \\
\hline Djursland & Syddjurs & Ryomgaard & Bulky & 3.82 & 3.89 & 4.22 & 4.37 & 4.45 & 3.63 & 3.38 \\
\hline Djursland & Syddjurs & Ebeltoft & OW & 20.82 & 19.37 & 18.69 & 18.23 & 17.4 & 17.61 & 18.22 \\
\hline Djursland & Syddjurs & Hornslet & OW & 20.09 & 20.05 & 18.9 & 19.75 & 19.71 & 19.25 & 18.59 \\
\hline Djursland & Syddjurs & Knebel & OW & 25.68 & 24.17 & 23.09 & 24.32 & 22.13 & 23.91 & 22.97 \\
\hline Djursland & Syddjurs & Ryomgaard & OW & 24.99 & 24.01 & 21.05 & 22.08 & 20.64 & 21.29 & 20.65 \\
\hline Djursland & Norddjurs & Glesborg & Fibres & 1.33 & 1.38 & 1.3 & 1.24 & 1.45 & 1.25 & 1.41 \\
\hline Djursland & Norddjurs & Grenaa & Fibres & 1.79 & 1.38 & 1.25 & 1.26 & 1.07 & 1.33 & 1.83 \\
\hline Djursland & Norddjurs & Roende & Fibres & 1.5 & 2.17 & 1.81 & 1.92 & 1.81 & 1.94 & 2.44 \\
\hline Djursland & Norddjurs & Tirstrup & Fibres & 1.34 & 0.93 & 1.36 & 1.47 & 1.51 & 1.35 & 2.07 \\
\hline Djursland & Norddjurs & Glesborg & Plastic & 0.89 & 0.77 & 1.08 & 1.13 & 1.12 & 1.08 & 1.13 \\
\hline Djursland & Norddjurs & Grenaa & Plastic & 0.69 & 0.81 & 0.65 & 0.72 & 0.63 & 0.75 & 0.85 \\
\hline Djursland & Norddjurs & Roende & Plastic & 0.54 & 0.81 & 0.77 & 0.85 & 0.89 & 0.82 & 0.97 \\
\hline Djursland & Norddjurs & Tirstrup & Plastic & 0.78 & 0.87 & 0.98 & 1.31 & 1.28 & 1.17 & 1.48 \\
\hline Djursland & Norddjurs & Glesborg & Metal & 5.11 & 5.15 & 5.42 & 4.95 & 5.11 & 5.37 & 5.69 \\
\hline Djursland & Norddjurs & Grenaa & Metal & 3.96 & 4.25 & 3.48 & 3.15 & 3.13 & 4.24 & 4.56 \\
\hline Djursland & Norddjurs & Roende & Metal & 3.37 & 4.24 & 3.99 & 4.41 & 4.57 & 4.77 & 4.86 \\
\hline
\end{tabular}




\begin{tabular}{|c|c|c|c|c|c|c|c|c|c|c|}
\hline Djursland & Norddjurs & Tirstrup & Metal & 5.07 & 5.54 & 4.12 & 4.94 & 5.1 & 5.41 & 6.29 \\
\hline Djursland & Norddjurs & Glesborg & Wood & 6.54 & 6.85 & 7.04 & 7.4 & 6.57 & 7.71 & 8.26 \\
\hline Djursland & Norddjurs & Grenaa & Wood & 7.55 & 7.69 & 6.64 & 6.31 & 5.87 & 7.49 & 9.1 \\
\hline Djursland & Norddjurs & Roende & Wood & 5.88 & 6.88 & 6.16 & 7.45 & 7.53 & 7.95 & 7.68 \\
\hline Djursland & Norddjurs & Tirstrup & Wood & 6.72 & 7.03 & 7.36 & 7.48 & 7.94 & 8.12 & 8.04 \\
\hline Djursland & Norddjurs & Glesborg & Glass & 0.7 & 1.53 & 1.73 & 1.92 & 2.15 & 2.25 & 1.87 \\
\hline Djursland & Norddjurs & Grenaa & Glass & 0.68 & 1.29 & 1.43 & 1.27 & 1.27 & 1.54 & 1.85 \\
\hline Djursland & Norddjurs & Roende & Glass & 0.7 & 2.24 & 3.17 & 2.32 & 1.99 & 2.03 & 2.77 \\
\hline Djursland & Norddjurs & Tirstrup & Glass & 0.52 & 1.39 & 1.48 & 1.91 & 1.48 & 2.45 & 2.71 \\
\hline Djursland & Norddjurs & Glesborg & Garden & 31.33 & 32.62 & 33.09 & 33.38 & 37.15 & 34.32 & 36.47 \\
\hline Djursland & Norddjurs & Grenaa & Garden & 24.19 & 24.94 & 35.27 & 42.12 & 47.47 & 35.24 & 29.77 \\
\hline Djursland & Norddjurs & Roende & Garden & 29.46 & 32.32 & 32.66 & 31.3 & 33.01 & 31.89 & 31.42 \\
\hline Djursland & Norddjurs & Tirstrup & Garden & 29.63 & 29.52 & 29.53 & 28.39 & 29.92 & 27.29 & 29.45 \\
\hline Djursland & Norddjurs & Glesborg & Soil & 3.09 & 2.18 & 3.41 & 3.19 & 3.12 & 3.1 & 3.59 \\
\hline Djursland & Norddjurs & Grenaa & Soil & 13.42 & 11.24 & 10.09 & 8.92 & 7.47 & 10.39 & 10.89 \\
\hline Djursland & Norddjurs & Roende & Soil & 14.1 & 7.98 & 8.84 & 6.51 & 9.33 & 7.84 & 7.79 \\
\hline Djursland & Norddjurs & Tirstrup & Soil & 5.27 & 4.15 & 6.04 & 4.28 & 4.64 & 4.97 & 3.34 \\
\hline Djursland & Norddjurs & Glesborg & $C \& D$ & 12.79 & 13.99 & 14.12 & 13.65 & 11.95 & 13.37 & 11.65 \\
\hline Djursland & Norddjurs & Grenaa & $C \& D$ & 15.92 & 17.6 & 16.07 & 13.63 & 13.26 & 14.6 & 14.9 \\
\hline Djursland & Norddjurs & Roende & $C \& D$ & 15.4 & 15.53 & 15.99 & 16.73 & 15.33 & 16.23 & 14.63 \\
\hline Djursland & Norddjurs & Tirstrup & $C \& D$ & 16.88 & 15.43 & 16.8 & 16.24 & 16.56 & 16.79 & 12.87 \\
\hline Djursland & Norddjurs & Glesborg & SMC & 6.58 & 6.24 & 6.15 & 6.21 & 5.44 & 5.42 & 5.19 \\
\hline Djursland & Norddjurs & Grenaa & SMC & 5.52 & 5.71 & 4.75 & 4.23 & 3.72 & 4.6 & 4.83 \\
\hline Djursland & Norddjurs & Roende & SMC & 5.76 & 5.72 & 5.64 & 6.21 & 5.73 & 5.47 & 5.41 \\
\hline Djursland & Norddjurs & Tirstrup & SMC & 6.64 & 6.39 & 6.28 & 6.32 & 6.13 & 5.62 & 5.9 \\
\hline Djursland & Norddjurs & Glesborg & Bulky & 4.27 & 3.8 & 3.78 & 3.57 & 3.34 & 3.55 & 3.74 \\
\hline Djursland & Norddjurs & Grenaa & Bulky & 3.33 & 3.33 & 2.92 & 2.51 & 2.22 & 2.62 & 3.15 \\
\hline Djursland & Norddjurs & Roende & Bulky & 3.35 & 2.88 & 2.92 & 3.12 & 2.71 & 2.63 & 2.88 \\
\hline Djursland & Norddjurs & Tirstrup & Bulky & 3.17 & 2.45 & 2.83 & 2.89 & 2.75 & 2.39 & 2.86 \\
\hline Djursland & Norddjurs & Glesborg & OW & 27.37 & 25.49 & 22.87 & 23.36 & 22.6 & 22.57 & 21 \\
\hline Djursland & Norddjurs & Grenaa & OW & 22.96 & 21.76 & 17.45 & 15.89 & 13.89 & 17.2 & 18.27 \\
\hline Djursland & Norddjurs & Roende & OW & 19.94 & 19.25 & 18.05 & 19.19 & 17.1 & 18.44 & 19.15 \\
\hline
\end{tabular}




\begin{tabular}{|c|c|c|c|c|c|c|c|c|c|c|}
\hline Djursland & Norddjurs & Tirstrup & OW & 23.97 & 26.3 & 23.21 & 24.76 & 22.69 & 24.44 & 24.98 \\
\hline Silkeborg & Silkeborg & Gjern & Fibres & 2.18 & 2.56 & 3.68 & 3.7 & 4 & 3.3 & 3.72 \\
\hline Silkeborg & Silkeborg & Tandskov & Fibres & 2.65 & 2.78 & 3.35 & 3.22 & 3.73 & 3.34 & 3.46 \\
\hline Silkeborg & Silkeborg & Them & Fibres & 3.02 & 2.74 & 3.17 & 3.97 & 3.89 & 4.17 & 3.63 \\
\hline Silkeborg & Silkeborg & Tietgensvej & Fibres & 5.41 & 2.89 & 3.53 & 4.68 & 4.5 & 3.85 & 4.35 \\
\hline Silkeborg & Silkeborg & Gjern & Plastic & 0.07 & 0.08 & 1.1 & 1.07 & 1.29 & 1.46 & 1.62 \\
\hline Silkeborg & Silkeborg & Tandskov & Plastic & 0.11 & 0.16 & 1.15 & 1.07 & 1.14 & 1.27 & 1.17 \\
\hline Silkeborg & Silkeborg & Them & Plastic & 0 & 0 & 0.73 & 1.7 & 1.36 & 1.32 & 1.24 \\
\hline Silkeborg & Silkeborg & Tietgensvej & Plastic & 0.25 & 0.22 & 0.92 & 1.11 & 1.13 & 1.18 & 1.21 \\
\hline Silkeborg & Silkeborg & Gjern & Metal & 5.12 & 4.9 & 4.79 & 4.44 & 4.16 & 4.76 & 4.47 \\
\hline Silkeborg & Silkeborg & Tandskov & Metal & 3.98 & 3.81 & 3.56 & 3.78 & 3.59 & 3.44 & 4.01 \\
\hline Silkeborg & Silkeborg & Them & Metal & 5.27 & 5.27 & 4.39 & 4.71 & 4.11 & 4.75 & 4.64 \\
\hline Silkeborg & Silkeborg & Tietgensvej & Metal & 3.87 & 3.75 & 3.87 & 3.64 & 3.63 & 3.79 & 3.66 \\
\hline Silkeborg & Silkeborg & Gjern & Wood & 4.24 & 9.88 & 10.14 & 10.61 & 11.77 & 12.78 & 13.26 \\
\hline Silkeborg & Silkeborg & Tandskov & Wood & 6.32 & 8.24 & 9.66 & 10.45 & 10.42 & 10.07 & 9.57 \\
\hline Silkeborg & Silkeborg & Them & Wood & 6.1 & 7.08 & 10.01 & 10.77 & 12.4 & 11.28 & 12.2 \\
\hline Silkeborg & Silkeborg & Tietgensvej & Wood & 6.2 & 8.22 & 9.42 & 11.29 & 11.82 & 11.78 & 11.84 \\
\hline Silkeborg & Silkeborg & Gjern & Glass & 0.96 & 1.99 & 2.19 & 2.13 & 2.34 & 2.28 & 2.25 \\
\hline Silkeborg & Silkeborg & Tandskov & Glass & 1.75 & 1.97 & 2.14 & 2.24 & 2.2 & 2.08 & 2.15 \\
\hline Silkeborg & Silkeborg & Them & Glass & 0 & 0 & 2.81 & 2.87 & 2.75 & 2.84 & 2.59 \\
\hline Silkeborg & Silkeborg & Tietgensvej & Glass & 2.29 & 2.6 & 2.89 & 2.8 & 2.74 & 2.77 & 2.74 \\
\hline Silkeborg & Silkeborg & Gjern & Garden & 24.05 & 23.86 & 24.34 & 23.22 & 24.55 & 26.17 & 25.42 \\
\hline Silkeborg & Silkeborg & Tandskov & Garden & 25.17 & 26.35 & 28.54 & 28.28 & 29.54 & 28.34 & 27.33 \\
\hline Silkeborg & Silkeborg & Them & Garden & 29.19 & 30.12 & 30.8 & 27.32 & 26.66 & 27.41 & 27.66 \\
\hline Silkeborg & Silkeborg & Tietgensvej & Garden & 26.54 & 27.76 & 28.37 & 25.31 & 26.88 & 27.21 & 26.91 \\
\hline Silkeborg & Silkeborg & Gjern & Soil & 8.43 & 8.43 & 12 & 10.55 & 10.51 & 10.46 & 10.42 \\
\hline Silkeborg & Silkeborg & Tandskov & Soil & 9.15 & 10.66 & 10.43 & 9.48 & 10.46 & 11.5 & 10.78 \\
\hline Silkeborg & Silkeborg & Them & Soil & 13.39 & 11.38 & 11.67 & 12.13 & 12.99 & 11.63 & 11.68 \\
\hline Silkeborg & Silkeborg & Tietgensvej & Soil & 11.07 & 10.66 & 10.57 & 13.22 & 13.14 & 11.98 & 11.5 \\
\hline Silkeborg & Silkeborg & Gjern & $\mathrm{C} \& \mathrm{D}$ & 25.64 & 26.37 & 22.99 & 25.97 & 24.93 & 22.32 & 22.99 \\
\hline Silkeborg & Silkeborg & Tandskov & $C \& D$ & 20.14 & 23.34 & 22.54 & 21.55 & 19.97 & 22.2 & 21.68 \\
\hline Silkeborg & Silkeborg & Them & C\&D & 17.91 & 18.02 & 18.66 & 20.08 & 21.68 & 21.23 & 22.15 \\
\hline
\end{tabular}




\begin{tabular}{|c|c|c|c|c|c|c|c|c|c|c|}
\hline Silkeborg & Silkeborg & Tietgensvej & $C \& D$ & 20.33 & 21.11 & 21.34 & 20.5 & 20.54 & 21.39 & 22.45 \\
\hline Silkeborg & Silkeborg & Gjern & SMC & 10.46 & 9.86 & 7.94 & 7.88 & 7.27 & 6.38 & 5.53 \\
\hline Silkeborg & Silkeborg & Tandskov & SMC & 9.89 & 9.33 & 7.12 & 7.56 & 6.77 & 6.57 & 6.16 \\
\hline Silkeborg & Silkeborg & Them & SMC & 10.26 & 10.89 & 7.73 & 7.19 & 5.89 & 6.22 & 4.65 \\
\hline Silkeborg & Silkeborg & Tietgensvej & SMC & 11.12 & 10.97 & 9.54 & 8.78 & 7.38 & 7.02 & 6.5 \\
\hline Silkeborg & Silkeborg & Gjern & Bulky & 8.71 & 4.26 & 3.66 & 3.29 & 2.9 & 3.66 & 3.97 \\
\hline Silkeborg & Silkeborg & Tandskov & Bulky & 6.92 & 4.91 & 3.54 & 3.58 & 3.44 & 3.54 & 3.98 \\
\hline Silkeborg & Silkeborg & Them & Bulky & 8.37 & 7.57 & 3.38 & 2.84 & 2.39 & 3.09 & 3.08 \\
\hline Silkeborg & Silkeborg & Tietgensvej & Bulky & 5.43 & 4.5 & 3.05 & 2.43 & 2.16 & 2.55 & 2.62 \\
\hline Silkeborg & Silkeborg & Gjern & OW & 10.14 & 7.8 & 7.18 & 7.14 & 6.29 & 6.42 & 6.36 \\
\hline Silkeborg & Silkeborg & Tandskov & OW & 13.94 & 8.45 & 7.97 & 8.78 & 8.74 & 7.64 & 9.72 \\
\hline Silkeborg & Silkeborg & Them & OW & 6.5 & 6.93 & 6.66 & 6.42 & 5.87 & 6.07 & 6.49 \\
\hline Silkeborg & Silkeborg & Tietgensvej & OW & 7.48 & 7.32 & 6.49 & 6.22 & 6.07 & 6.47 & 6.23 \\
\hline
\end{tabular}

Table S15: Detail composition based on 59 waste fractions for each regions from 2010 to 2016 (wet mass composition \%)

\begin{tabular}{|c|c|c|c|c|c|c|c|c|}
\hline Area & Fractions & 2010 & 2011 & 2012 & 2013 & 2014 & 2015 & 2016 \\
\hline \multirow[t]{17}{*}{ Greater Copenhagen } & Anti-freeze & 0.000 & 0.000 & 0.000 & 0.000 & 0.000 & 0.000 & 0.000 \\
\hline & Asbestos waste & 0.600 & 0.600 & 0.600 & 0.600 & 0.500 & 0.500 & 0.500 \\
\hline & Asphalt residues & 0.100 & 0.600 & 0.500 & 0.400 & 0.400 & 0.500 & 0.300 \\
\hline & Batteries & 0.100 & 0.100 & 0.100 & 0.100 & 0.100 & 0.100 & 0.100 \\
\hline & Brics & 0.000 & 0.000 & 0.000 & 0.100 & 1.200 & 2.400 & 2.400 \\
\hline & Brics, blocks and tiles & 10.700 & 0.200 & 0.200 & 0.400 & 0.300 & 0.300 & 0.300 \\
\hline & Bulky & 7.900 & 1.200 & 0.400 & 0.000 & 0.000 & 0.000 & 0.000 \\
\hline & Cables & 0.000 & 0.000 & 0.000 & 0.000 & 0.100 & 0.100 & 0.100 \\
\hline & Chemichal fertilizer & 0.000 & 0.000 & 0.000 & 0.000 & 0.000 & 0.000 & 0.000 \\
\hline & Clinical waste & 0.000 & 0.000 & 0.000 & 0.000 & 0.000 & 0.000 & 0.000 \\
\hline & Concrete & 2.300 & 12.600 & 13.000 & 12.100 & 12.700 & 12.900 & 12.400 \\
\hline & Cooling and heating white goods & 1.000 & 0.900 & 1.000 & 1.000 & 0.900 & 1.100 & 1.100 \\
\hline & Dense plastics & 0.000 & 0.000 & 0.200 & 0.700 & 0.800 & 0.800 & 0.900 \\
\hline & Fire extinguisher & 0.000 & 0.000 & 0.000 & 0.000 & 0.000 & 0.000 & 0.000 \\
\hline & Fireworks & 0.000 & 0.000 & 0.000 & 0.000 & 0.000 & 0.000 & 0.000 \\
\hline & Furniture & 0.100 & 0.000 & 0.000 & 1.300 & 1.400 & 1.300 & 1.400 \\
\hline & Garden herbicides and pesticides & 0.000 & 0.000 & 0.000 & 0.000 & 0.000 & 0.000 & 0.000 \\
\hline
\end{tabular}




\begin{tabular}{|c|c|c|c|c|c|c|c|c|}
\hline & Garden waste & 12.300 & 12.900 & 11.900 & 12.600 & 13.000 & 12.600 & 12.900 \\
\hline & Gas bottles & 0.000 & 0.000 & 0.000 & 0.000 & 0.000 & 0.000 & 0.000 \\
\hline & Glass packaging & 0.300 & 0.200 & 0.000 & 0.000 & 0.000 & 0.000 & 0.000 \\
\hline & Gypsum waste & 2.500 & 2.500 & 2.300 & 2.400 & 2.500 & 2.400 & 2.500 \\
\hline & Landfilled waste & 10.300 & 1.300 & 1.300 & 1.400 & 1.400 & 1.300 & 2.200 \\
\hline & Lighting equipment & 0.000 & 0.000 & 0.000 & 0.000 & 0.000 & 0.000 & 0.000 \\
\hline & Mattresses & 0.400 & 0.600 & 0.900 & 0.100 & 0.000 & 0.000 & 0.000 \\
\hline & Metal containers and cans & 4.700 & 4.400 & 4.200 & 4.100 & 3.800 & 3.800 & 4.100 \\
\hline & Mineral oil & 0.000 & 0.000 & 0.000 & 0.000 & 0.000 & 0.200 & 0.600 \\
\hline & Miscellaneous combustible & 12.400 & 11.000 & 9.600 & 9.400 & 8.700 & 8.100 & 7.800 \\
\hline & Other wood & 0.600 & 0.500 & 0.600 & 0.300 & 0.000 & 0.000 & 0.000 \\
\hline & Paints & 0.400 & 0.400 & 0.500 & 0.500 & 0.600 & 0.600 & 0.700 \\
\hline & Paper and board & 2.600 & 2.400 & 2.300 & 2.400 & 2.300 & 2.200 & 2.400 \\
\hline & Plastic packaging & 0.100 & 0.100 & 0.100 & 0.100 & 0.100 & 0.100 & 0.100 \\
\hline & Plastics films & 0.000 & 0.000 & 0.000 & 0.000 & 0.000 & 0.000 & 0.000 \\
\hline & Polystyrene & 0.000 & 0.000 & 0.000 & 0.000 & 0.000 & 0.000 & 0.000 \\
\hline & Polyvinylchloride (PVC) & 0.200 & 0.200 & 0.100 & 0.000 & 0.000 & 0.000 & 0.000 \\
\hline & Printer cartridges & 0.000 & 0.000 & 0.000 & 0.000 & 0.000 & 0.000 & 0.000 \\
\hline & Sanitary ware & 3.100 & 7.400 & 8.100 & 8.300 & 8.900 & 9.300 & 8.300 \\
\hline & Small household appliances & 1.600 & 1.500 & 1.400 & 1.400 & 1.200 & 1.200 & 1.100 \\
\hline & Soil & 14.100 & 14.000 & 15.400 & 14.100 & 14.500 & 14.500 & 13.900 \\
\hline & Solvent-based waste & 0.000 & 0.000 & 0.000 & 0.000 & 0.000 & 0.000 & 0.000 \\
\hline & Spray can & 0.000 & 0.000 & 0.000 & 0.000 & 0.000 & 0.000 & 0.000 \\
\hline & Textiles & 0.200 & 0.200 & 0.300 & 0.400 & 0.400 & 0.400 & 0.500 \\
\hline & Tiles & 5.700 & 8.200 & 8.400 & 8.300 & 6.100 & 4.300 & 4.300 \\
\hline & Treated wood & 0.100 & 0.200 & 0.200 & 0.200 & 0.200 & 0.300 & 0.300 \\
\hline & Tyres & 0.200 & 0.200 & 0.200 & 0.200 & 0.200 & 0.200 & 0.200 \\
\hline & Untreated wood & 4.300 & 14.300 & 15.000 & 15.800 & 16.100 & 16.900 & 17.300 \\
\hline & Waste exchange & 0.000 & 0.000 & 0.000 & 0.100 & 0.600 & 0.600 & 0.300 \\
\hline & Windows glass & 1.100 & 1.200 & 1.100 & 1.100 & 1.000 & 1.100 & 1.000 \\
\hline & Windows glass with PCB & 0.000 & 0.000 & 0.100 & 0.000 & 0.000 & 0.000 & 0.000 \\
\hline & & 100 & 100 & 100 & 100 & 100 & 100 & 100 \\
\hline Djursland & Asbestos waste & 3.500 & 4.100 & 3.400 & 3.100 & 3.100 & 3.400 & 3.200 \\
\hline & Brics & 0.000 & 0.000 & 0.000 & 0.000 & 0.000 & 0.300 & 1.200 \\
\hline & Bulky & 3.500 & 3.300 & 3.300 & 3.300 & 3.000 & 3.000 & 3.200 \\
\hline & Cables & 0.100 & 0.100 & 0.100 & 0.100 & 0.100 & 0.100 & 0.100 \\
\hline & Clinical waste & 0.000 & 0.000 & 0.000 & 0.000 & 0.000 & 0.000 & 0.000 \\
\hline & Concrete & 13.700 & 15.000 & 14.200 & 13.600 & 13.100 & 13.100 & 11.500 \\
\hline & Cooling and heating white goods & 1.500 & 1.300 & 1.400 & 1.700 & 1.200 & 1.400 & 1.100 \\
\hline & Dense plastics & 0.600 & 0.700 & 0.700 & 0.700 & 0.700 & 0.700 & 0.800 \\
\hline & Ferrous metal & 4.300 & 4.700 & 4.400 & 4.400 & 4.400 & 4.900 & 5.000 \\
\hline & Garden waste & 28.500 & 29.600 & 33.100 & 34.400 & 37.500 & 33.300 & 32.300 \\
\hline
\end{tabular}




\begin{tabular}{|c|c|c|c|c|c|c|c|c|}
\hline & Gas bottles & 0.100 & 0.100 & 0.200 & 0.100 & 0.100 & 0.000 & 0.000 \\
\hline & Glass packaging & 0.300 & 0.400 & 0.300 & 0.400 & 0.300 & 0.600 & 0.800 \\
\hline & Gypsum waste & 1.100 & 1.100 & 1.000 & 1.000 & 0.900 & 1.100 & 1.000 \\
\hline & Landfilled waste & 11.900 & 10.700 & 9.600 & 8.600 & 7.500 & 7.800 & 7.700 \\
\hline & Miscellaneous combustible & 6.000 & 6.100 & 5.800 & 5.800 & 5.400 & 5.500 & 5.500 \\
\hline & Paper and board & 1.900 & 1.900 & 1.800 & 1.800 & 1.700 & 1.800 & 1.900 \\
\hline & Plastics films & 0.100 & 0.200 & 0.200 & 0.300 & 0.200 & 0.200 & 0.300 \\
\hline & Polyvinylchloride (PVC) & 0.200 & 0.000 & 0.200 & 0.200 & 0.200 & 0.200 & 0.200 \\
\hline & Small household appliances & 1.500 & 1.500 & 0.400 & 1.600 & 1.300 & 1.500 & 1.800 \\
\hline & Soil & 9.700 & 6.900 & 7.000 & 6.000 & 6.200 & 6.400 & 6.500 \\
\hline & Textiles & 0.000 & 0.000 & 0.000 & 0.000 & 0.000 & 0.000 & 0.300 \\
\hline & Thermal insulation materials & 0.000 & 0.000 & 0.000 & 0.200 & 0.300 & 0.300 & 0.300 \\
\hline & Treated wood & 3.700 & 4.000 & 4.000 & 3.900 & 4.200 & 4.600 & 4.900 \\
\hline & Tires & 0.300 & 0.300 & 0.300 & 0.300 & 0.300 & 0.400 & 0.300 \\
\hline & Untreated wood & 7.000 & 7.100 & 7.000 & 7.200 & 7.000 & 7.900 & 8.700 \\
\hline & Waste accumulators & 0.100 & 0.100 & 0.100 & 0.100 & 0.100 & 0.100 & 0.100 \\
\hline & Windows glass & 0.400 & 1.200 & 1.500 & 1.400 & 1.400 & 1.300 & 1.400 \\
\hline & & 100 & 100 & 100 & 100 & 100 & 100 & 100 \\
\hline \multirow[t]{24}{*}{ Central Zealand } & Asbestos waste & 2.100 & 1.900 & 1.700 & 1.800 & 1.900 & 1.600 & 1.700 \\
\hline & Asphalt residues & 0.100 & 0.200 & 0.100 & 0.200 & 0.200 & 0.200 & 0.100 \\
\hline & Bigbags & 0.000 & 0.000 & 0.000 & 0.000 & 0.000 & 0.000 & 0.000 \\
\hline & Brics, blocks and tiles & 12.300 & 15.000 & 21.100 & 17.400 & 19.500 & 20.300 & 20.100 \\
\hline & Bulky & 11.700 & 8.400 & 12.900 & 13.100 & 11.900 & 9.500 & 7.900 \\
\hline & Cables & 0.000 & 0.000 & 0.000 & 0.000 & 0.000 & 0.000 & 0.100 \\
\hline & Concrete & 10.200 & 7.100 & 1.400 & 4.200 & 3.000 & 2.800 & 2.800 \\
\hline & Ferrous metal & 4.100 & 4.300 & 4.300 & 3.900 & 4.300 & 4.300 & 4.600 \\
\hline & Garden waste & 26.000 & 24.200 & 25.700 & 25.300 & 25.800 & 25.500 & 25.900 \\
\hline & Gas bottles & 0.100 & 0.100 & 0.000 & 0.600 & 0.100 & 0.000 & 0.000 \\
\hline & Glass packaging & 1.000 & 1.100 & 1.300 & 0.900 & 0.900 & 1.000 & 0.700 \\
\hline & Gypsum waste & 1.500 & 1.400 & 1.400 & 1.500 & 1.500 & 1.600 & 1.600 \\
\hline & Landfilled waste & 2.900 & 2.400 & 1.800 & 1.200 & 0.900 & 0.700 & 0.600 \\
\hline & Large household Appliances & 0.400 & 0.900 & 0.700 & 2.600 & 0.100 & 0.200 & 0.200 \\
\hline & Mineral oil & 0.800 & 0.600 & 0.900 & 1.300 & 1.200 & 1.200 & 1.100 \\
\hline & Miscellaneous combustible & 12.000 & 17.900 & 12.300 & 12.600 & 13.600 & 14.200 & 11.100 \\
\hline & Mixed waste & 0.000 & 0.100 & 0.000 & 0.000 & 0.000 & 0.000 & 0.000 \\
\hline & Non-ferrous metal & 0.000 & 0.000 & 0.000 & 0.000 & 0.000 & 0.000 & 0.000 \\
\hline & Paper and board & 2.300 & 2.000 & 2.100 & 2.100 & 1.900 & 1.900 & 1.800 \\
\hline & Pastics composite & 0.000 & 0.000 & 0.000 & 0.000 & 0.000 & 0.000 & 0.000 \\
\hline & Plastics films & 0.000 & 0.000 & 0.000 & 0.100 & 0.100 & 0.100 & 0.100 \\
\hline & Polyvinylchloride (PVC) & 0.300 & 0.200 & 0.300 & 0.400 & 0.500 & 0.500 & 0.500 \\
\hline & Residual Household waste & 0.100 & 0.100 & 0.000 & 0.000 & 0.000 & 0.000 & 0.000 \\
\hline & Sanitary ware & 0.000 & 0.000 & 0.000 & 0.700 & 0.900 & 0.900 & 0.800 \\
\hline
\end{tabular}




\begin{tabular}{|c|c|c|c|c|c|c|c|c|}
\hline & Slugde & 0.000 & 0.000 & 0.000 & 0.000 & 0.000 & 0.000 & 0.000 \\
\hline & Small household appliances & 0.500 & 1.400 & 1.000 & 0.000 & 0.000 & 0.000 & 0.000 \\
\hline & Soil & 8.600 & 7.800 & 7.900 & 7.300 & 8.200 & 8.100 & 8.100 \\
\hline & Treated wood & 1.300 & 1.200 & 1.200 & 1.300 & 1.500 & 1.400 & 1.700 \\
\hline & Tires & 0.400 & 0.400 & 0.300 & 0.200 & 0.400 & 0.400 & 0.400 \\
\hline & Untreated wood & 0.100 & 0.000 & 0.000 & 0.000 & 0.000 & 2.300 & 6.600 \\
\hline & Waste accumulators & 0.000 & 0.000 & 0.000 & 0.000 & 0.000 & 0.100 & 0.100 \\
\hline & Windows glass & 1.200 & 1.200 & 1.400 & 1.400 & 1.400 & 1.500 & 1.300 \\
\hline & & 100 & 100 & 100 & 100 & 100 & 100 & 100 \\
\hline \multirow[t]{31}{*}{ Silkeborg } & Asbestos waste & 2.400 & 0.500 & 0.500 & 0.600 & 0.500 & 0.400 & 1.100 \\
\hline & Batteries & 0.000 & 0.100 & 0.000 & 0.100 & 0.100 & 0.100 & 0.100 \\
\hline & Brics, blocks and tiles & 19.500 & 20.400 & 19.400 & 19.000 & 18.600 & 19.200 & 19.700 \\
\hline & Bulky & 6.700 & 5.000 & 3.400 & 3.000 & 2.800 & 3.100 & 3.400 \\
\hline & Cables & 0.000 & 0.000 & 0.100 & 0.100 & 0.100 & 0.100 & 0.100 \\
\hline & CDs and DVDs & 0.000 & 0.000 & 0.000 & 0.000 & 0.000 & 0.000 & 0.000 \\
\hline & Dense plastics & 0.100 & 0.100 & 0.700 & 0.800 & 0.700 & 0.700 & 0.800 \\
\hline & Ferrous metal & 4.000 & 3.900 & 3.700 & 3.700 & 3.600 & 3.800 & 4.000 \\
\hline & Garden waste & 26.000 & 27.000 & 28.300 & 26.500 & 27.700 & 27.600 & 27.000 \\
\hline & Glass packaging & 1.000 & 1.200 & 1.500 & 1.500 & 1.400 & 1.500 & 1.500 \\
\hline & Gypsum waste & 0.900 & 1.000 & 1.100 & 1.100 & 1.000 & 1.100 & 1.100 \\
\hline & Landfilled waste & 4.300 & 2.600 & 1.900 & 1.900 & 1.900 & 1.800 & 1.700 \\
\hline & Metal containers and cans & 0.100 & 0.100 & 0.100 & 0.200 & 0.100 & 0.000 & 0.000 \\
\hline & Miscellaneous combustible & 10.500 & 10.100 & 8.100 & 8.000 & 6.900 & 6.700 & 6.000 \\
\hline & Paper and board & 3.500 & 2.500 & 3.000 & 3.400 & 3.600 & 3.200 & 3.400 \\
\hline & Plastics films & 0.000 & 0.000 & 0.200 & 0.300 & 0.300 & 0.400 & 0.300 \\
\hline & Plastics garden furniture & 0.000 & 0.000 & 0.000 & 0.000 & 0.100 & 0.100 & 0.100 \\
\hline & Polystyrene & 0.000 & 0.000 & 0.100 & 0.100 & 0.100 & 0.000 & 0.000 \\
\hline & Polyvinylchloride (PVC) & 0.100 & 0.200 & 0.200 & 0.200 & 0.200 & 0.200 & 0.200 \\
\hline & Sanitary ware & 0.100 & 0.900 & 1.200 & 1.400 & 1.300 & 1.500 & 1.400 \\
\hline & Small household appliances & 1.700 & 2.700 & 3.000 & 3.000 & 2.700 & 2.600 & 2.500 \\
\hline & Soil & 10.300 & 10.500 & 10.800 & 11.300 & 11.700 & 11.600 & 11.100 \\
\hline & Textiles & 0.200 & 0.300 & 0.500 & 0.500 & 0.500 & 0.500 & 0.400 \\
\hline & Thermal insulation materials & 0.000 & 0.000 & 0.000 & 0.000 & 0.000 & 0.000 & 0.000 \\
\hline & Treated wood & 1.300 & 1.300 & 1.100 & 1.200 & 1.400 & 1.400 & 1.600 \\
\hline & Tyres & 0.400 & 0.400 & 0.400 & 0.400 & 0.300 & 0.300 & 0.400 \\
\hline & Untreated wood & 6.000 & 8.300 & 9.700 & 10.800 & 11.300 & 11.100 & 11.100 \\
\hline & Waste accumulators & 0.100 & 0.100 & 0.100 & 0.100 & 0.100 & 0.100 & 0.100 \\
\hline & Windows glass & 0.700 & 0.800 & 1.000 & 1.000 & 1.000 & 0.900 & 0.900 \\
\hline & Windows glass with $\mathrm{PCB}$ & 0.000 & 0.000 & 0.000 & 0.000 & 0.000 & 0.000 & 0.000 \\
\hline & & 100 & 100 & 100 & 100 & 100 & 100 & 100 \\
\hline
\end{tabular}



Table S16: Distribution of plastic bags used to bring the waste at the household waste recycling centres

\begin{tabular}{lcccc}
\hline \multirow{2}{*}{$\begin{array}{l}\text { Household waste Recycling } \\
\text { centers }\end{array}$} & Without & Mixed & Clear & Black \\
\cline { 2 - 5 } Dalskov & 50 & 18 & 23 & 9 \\
Hvidovre & 41 & 19 & 36 & 3 \\
Hvidovre_II & 42 & 24 & 26 & 7 \\
Jyllinge & 56 & 18 & 19 & 7 \\
Lemvig & 53 & 26 & 11 & 10 \\
Tandskov & 55 & 19 & 21 & 6 \\
Viby & 57 & 12 & 27 & 5 \\
\hline
\end{tabular}

Table S17: Summary of miscellaneous waste composition based on disposal waste containers

\begin{tabular}{lcccc}
\hline \multirow{2}{*}{ Parameters } & \multicolumn{3}{c}{ Type of waste containers } & \\
\cline { 2 - 5 } n & Mixed transparent & Loose & Transparent & Black \\
vars & 7.00 & 7.00 & 7.00 & 7.00 \\
maximum & 1.00 & 1.00 & 1.00 & 1.00 \\
mean & 25.90 & 56.80 & 36.00 & 9.60 \\
median & 19.36 & 50.53 & 23.41 & 6.69 \\
minimum & 18.80 & 53.00 & 23.50 & 6.90 \\
q1 & 11.90 & 41.50 & 11.50 & 3.30 \\
q3 & 17.65 & 45.90 & 20.00 & 5.35 \\
kurtosis & 21.80 & 55.30 & 26.45 & 8.15 \\
mad & -1.29 & -1.75 & -1.00 & -1.59 \\
max & 1.78 & 4.60 & 4.60 & 2.82 \\
range & 25.90 & 56.80 & 36.00 & 9.60 \\
sd & 14.00 & 15.30 & 24.50 & 6.30 \\
se & 4.64 & 6.43 & 7.56 & 2.22 \\
skew & 1.75 & 2.43 & 2.86 & 0.84 \\
trimmed & -0.03 & -0.48 & 0.09 & -0.16 \\
\hline
\end{tabular}

Table S18: Summary of misplaced waste in small miscellaneous combustible waste

\begin{tabular}{lcc}
\hline Descriptive statistics & Misplaced & Small Miscellaneous Combustible \\
\hline $\mathrm{n}$ & 7 & 7 \\
vars & 1 & 1 \\
mean & 67.5 & 32.5 \\
sd & 9.7 & 9.7 \\
median & 64.2 & 35.8 \\
mad & 12.5 & 12.5 \\
trimmed & 67.5 & 32.5 \\
min & 55.8 & 19.0 \\
max & 81.0 & 44.2 \\
range & 25.2 & 25.2 \\
kurtosis & -1.9 & -1.9 \\
skew & 0.1 & -0.1 \\
se & 3.7 & 3.7 \\
\hline
\end{tabular}


Table S19 Univariate Box-Pierce test considering percentage composition

\begin{tabular}{|c|c|c|c|c|c|c|c|c|c|}
\hline Affaldsselskab & Kommune & HWRCs & Bulky & C\&D & Garden & OW & $\mathrm{SC}$ & Soil & TWF \\
\hline \multirow[b]{8}{*}{ Djursland } & Norddjurs & Glesborg & 0.0725 & 0.4736 & 0.0000 & 0.0001 & 0.0002 & 0.5821 & 0.0001 \\
\hline & Norddjurs & Grenaa & 0.2808 & 0.2240 & 0.1022 & 0.3186 & 0.0153 & 0.6070 & 0.1637 \\
\hline & Norddjurs & Roende & 0.0001 & 0.0014 & 0.0000 & 0.6070 & 0.0000 & 0.8818 & 0.0001 \\
\hline & Norddjurs & Tirstrup & 0.1176 & 0.0216 & 0.0311 & 0.3476 & 0.0004 & 0.8612 & 0.5491 \\
\hline & Syddjurs & Ebeltoft & 0.0090 & 0.0503 & 0.0000 & 0.0222 & 0.0000 & 0.7402 & 0.0005 \\
\hline & Syddjurs & Hornslet & 0.0000 & 0.6389 & 0.0000 & 0.0023 & 0.0000 & 0.2535 & 0.0017 \\
\hline & Syddjurs & Knebel & 0.8816 & 0.9647 & 0.0000 & 0.1518 & 0.0000 & 0.5222 & 0.0439 \\
\hline & Syddjurs & Ryomgaard & 0.0130 & 0.0231 & 0.0000 & 0.0000 & 0.0000 & 0.7626 & 0.0000 \\
\hline \multirow{4}{*}{ Silkeborg } & Silkeborg & Tandskov & 0.0000 & 0.1054 & 0.0000 & 0.0011 & 0.0000 & 0.0011 & 0.0000 \\
\hline & Silkeborg & Tietgensvej & 0.0000 & 0.2715 & 0.0000 & 0.0000 & 0.0000 & 0.0000 & 0.0000 \\
\hline & Silkeborg & Gjern & 0.0000 & 0.1480 & 0.0000 & 0.0006 & 0.0000 & 0.0829 & 0.0000 \\
\hline & Silkeborg & Them & 0.0000 & 0.0269 & 0.0005 & 0.0286 & 0.0000 & 0.0051 & 0.0000 \\
\hline \multirow{10}{*}{$\begin{array}{l}\text { Greater } \\
\text { Copenhagen }\end{array}$} & Dragoer & Dragoer & 0.0000 & 0.0057 & 0.0000 & 0.0000 & 0.0000 & 0.0000 & 0.0000 \\
\hline & Frederiksberg & Bispeengen & 0.7922 & 0.4451 & 0.0000 & 0.1891 & 0.0550 & 0.0023 & 0.8770 \\
\hline & Hvidovre & Hvidovre & 0.0000 & 0.0000 & 0.0000 & 0.0000 & 0.0000 & 0.0000 & 0.0000 \\
\hline & Koebenhavn & Borgervaenget & 0.4133 & 0.0078 & 0.3887 & 0.0138 & 0.0602 & 0.9655 & 0.5983 \\
\hline & Koebenhavn & Chrisiania & 0.0000 & 0.2586 & 0.2339 & 0.0004 & 0.8880 & 0.0302 & 0.6199 \\
\hline & Koebenhavn & Kulbanevej & 0.0000 & 0.0000 & 0.0009 & 0.0000 & 0.0000 & 0.0000 & 0.0000 \\
\hline & Koebenhavn & Vasbygade & 0.0000 & 0.0004 & 0.0328 & 0.0000 & 0.0000 & 0.5113 & 0.0000 \\
\hline & Koebenhavn & Vermlandsgade & 0.0000 & 0.0000 & 0.0015 & 0.0000 & 0.0000 & 0.0000 & 0.0000 \\
\hline & Taarnby & Kirstinehoej & 0.0000 & 0.0000 & 0.0000 & 0.0000 & 0.0000 & 0.0000 & 0.0000 \\
\hline & Taarnby & Vaegtergangen & 0.0000 & 0.8406 & 0.0000 & 0.2754 & 0.0000 & 0.0984 & 0.0000 \\
\hline \multirow{14}{*}{ Central Zealand } & Kalundborg & Bregninge & 0.0000 & 0.0007 & 0.0000 & 0.0092 & 0.0002 & 0.0005 & 0.0000 \\
\hline & Kalundborg & Goerlev & 0.0439 & 0.1942 & 0.0000 & 0.0001 & 0.0007 & 0.0015 & 0.0000 \\
\hline & Kalundborg & Hoeng & 0.0008 & 0.6586 & 0.1207 & 0.2275 & 0.0011 & 0.0002 & 0.0001 \\
\hline & Kalundborg & Kalundborg & 0.0000 & 0.0038 & 0.0000 & 0.0523 & 0.0000 & 0.0004 & 0.0096 \\
\hline & Kalundborg & Sejeroe & 0.5824 & 0.0622 & 0.8210 & 0.2448 & 0.2808 & 0.0060 & 0.0553 \\
\hline & Kalundborg & Ubby & 0.4432 & 0.0434 & 0.0713 & 0.8824 & 0.0020 & 0.4779 & 0.1002 \\
\hline & Koege & Bjaeverskov & 0.0006 & 0.4046 & 0.0011 & 0.0003 & 0.0000 & 0.0651 & 0.5371 \\
\hline & Koege & Koege & 0.0000 & 0.1841 & 0.0002 & 0.3070 & 0.0000 & 0.0000 & 0.0140 \\
\hline & Lejre & Torkilstrup & 0.0000 & 0.4045 & 0.0000 & 0.0002 & 0.0000 & 0.0000 & 0.0000 \\
\hline & Roskilde & Jyllinge & 0.0000 & 0.2086 & 0.0009 & 0.2412 & 0.0006 & 0.0000 & 0.0004 \\
\hline & Roskilde & Roskilde & 0.0000 & 0.0892 & 0.0003 & 0.0544 & 0.0000 & 0.0000 & 0.0004 \\
\hline & Roskilde & Viby & 0.0002 & 0.7676 & 0.0065 & 0.0026 & 0.0033 & 0.0048 & 0.0003 \\
\hline & Stevns & Haarlev & 0.0059 & 0.1766 & 0.2734 & 0.0191 & 0.0004 & 0.0039 & 0.0000 \\
\hline & Stevns & Store_Hedding & 0.0003 & 0.0662 & 0.0003 & 0.0250 & 0.0001 & 0.0383 & 0.0000 \\
\hline
\end{tabular}


Table S20 Univariate Box-Pierce test considering waste generation (mass)

\begin{tabular}{|c|c|c|c|c|c|c|c|c|c|}
\hline Affaldsselskab & Kommune & Genbrugspladser & Bulky & C\&D & Garden & OW & $\mathrm{SC}$ & Soil & TWF \\
\hline \multirow[b]{8}{*}{ Djursland } & Norddjurs & Glesborg & 0.0029 & 0.0000 & 0.0000 & 0.0019 & 0.0002 & 0.8754 & 0.0058 \\
\hline & Norddjurs & Grenaa & 0.0004 & 0.0000 & 0.1755 & 0.0003 & 0.0003 & 0.8121 & 0.0043 \\
\hline & Norddjurs & Roende & 0.0000 & 0.0000 & 0.0000 & 0.0000 & 0.0000 & 0.8915 & 0.0000 \\
\hline & Norddjurs & Tirstrup & 0.0463 & 0.0000 & 0.0000 & 0.0166 & 0.0005 & 0.9727 & 0.0003 \\
\hline & Syddjurs & Ebeltoft & 0.0077 & 0.0000 & 0.0000 & 0.0035 & 0.0687 & 0.8886 & 0.0051 \\
\hline & Syddjurs & Hornslet & 0.0690 & 0.0000 & 0.0000 & 0.2558 & 0.0411 & 0.8057 & 0.0102 \\
\hline & Syddjurs & Knebel & 0.0001 & 0.0000 & 0.0000 & 0.0051 & 0.0868 & 0.7089 & 0.1349 \\
\hline & Syddjurs & Ryomgaard & 0.0053 & 0.0000 & 0.0000 & 0.0327 & 0.0102 & 0.8682 & 0.0020 \\
\hline \multirow{4}{*}{ Silkeborg } & Silkeborg & Tandskov & 0.0000 & 0.0000 & 0.0000 & 0.0004 & 0.0000 & 0.0000 & 0.0000 \\
\hline & Silkeborg & Tietgensvej & 0.0000 & 0.0000 & 0.0000 & 0.0079 & 0.0000 & 0.0000 & 0.0227 \\
\hline & Silkeborg & Gjern & 0.0000 & 0.0302 & 0.0000 & 0.9182 & 0.0267 & 0.0023 & 0.0000 \\
\hline & Silkeborg & Them & 0.0000 & 0.0174 & 0.0000 & 0.0457 & 0.0000 & 0.0015 & 2.4840 \\
\hline \multirow{10}{*}{$\begin{array}{l}\text { Greater } \\
\text { Copenhagen }\end{array}$} & Dragoer & Dragoer & 0.0000 & 0.0000 & 0.0000 & 0.0000 & 0.0001 & 0.0000 & 0.0000 \\
\hline & Frederiksberg & Bispeengen & 0.0086 & 0.0000 & 0.0000 & 0.0000 & 0.0000 & 0.0000 & 0.0000 \\
\hline & Hvidovre & Hvidovre & 0.0000 & 0.0000 & 0.0000 & 0.0000 & 0.0000 & 0.0000 & 0.0000 \\
\hline & Koebenhavn & Borgervaenget & 0.0049 & 0.0000 & 0.0325 & 0.4107 & 0.0016 & 0.0159 & 0.0000 \\
\hline & Koebenhavn & Chrisiania & 0.0049 & 0.7613 & 0.2002 & 0.0797 & 0.0038 & 0.0254 & 0.0174 \\
\hline & Koebenhavn & Kulbanevej & 0.0000 & 0.0000 & 0.0000 & 0.0000 & 0.0000 & 0.0000 & 0.0000 \\
\hline & Koebenhavn & Vasbygade & 0.0000 & 0.0000 & 0.7143 & 0.0000 & 0.0000 & 0.0063 & 0.0000 \\
\hline & Koebenhavn & Vermlandsgade & 0.0000 & 0.0000 & 0.0000 & 0.0000 & 0.0000 & 0.0000 & 0.0000 \\
\hline & Taarnby & Kirstinehoej & 0.0000 & 0.0000 & 0.0000 & 0.0000 & 0.0000 & 0.0000 & 0.0000 \\
\hline & Taarnby & Vaegtergangen & 0.0000 & 0.0021 & 0.0000 & 0.1496 & 0.0000 & 0.0458 & 0.0007 \\
\hline \multirow{14}{*}{ Roskilde } & Kalundborg & Bregninge & 0.0000 & 0.0000 & 0.0000 & 0.1628 & 0.0000 & 0.0000 & 0.0003 \\
\hline & Kalundborg & Goerlev & 0.0000 & 0.0200 & 0.0000 & 0.0006 & 0.0000 & 0.0000 & 0.0057 \\
\hline & Kalundborg & Hoeng & 0.0000 & 0.6949 & 0.0000 & 0.7201 & 0.0131 & 0.0000 & 0.0000 \\
\hline & Kalundborg & Kalundborg & 0.0000 & 0.0010 & 0.0000 & 0.0097 & 0.0000 & 0.0000 & 0.3676 \\
\hline & Kalundborg & Sejeroe & 0.5814 & 0.1841 & 0.8504 & 0.2030 & 0.6379 & 0.0004 & 0.3664 \\
\hline & Kalundborg & Ubby & 0.0009 & 0.0005 & 0.0000 & 0.4380 & 0.0192 & 0.0190 & 0.9741 \\
\hline & Koege & Bjaeverskov & 0.0057 & 0.0000 & 0.0000 & 0.1792 & 0.0008 & 0.0001 & 0.0003 \\
\hline & Koege & Koege & 0.0001 & 0.0087 & 0.0000 & 0.6597 & 0.0001 & 0.0000 & 0.0000 \\
\hline & Lejre & Torkilstrup & 0.0000 & 0.0113 & 0.0000 & 0.5975 & 0.0001 & 0.0000 & 0.0000 \\
\hline & Roskilde & Jyllinge & 0.0000 & 0.0000 & 0.0000 & 0.5271 & 0.0003 & 0.0000 & 0.0000 \\
\hline & Roskilde & Roskilde & 0.0001 & 0.0694 & 0.0000 & 0.8769 & 0.0000 & 0.0000 & 0.0000 \\
\hline & Roskilde & Viby & 0.0000 & 0.0078 & 0.0000 & 0.2960 & 0.0001 & 0.0000 & 0.0000 \\
\hline & Stevns & Haarlev & 0.0051 & 0.0215 & 0.1192 & 0.7459 & 0.0044 & 0.0002 & 0.0676 \\
\hline & Stevns & Store_Hedding & 0.0001 & 0.0805 & 0.0000 & 0.1183 & 0.0214 & 0.0001 & 0.0002 \\
\hline
\end{tabular}


Figure S1: Graph showing the number of visitors per households and per population

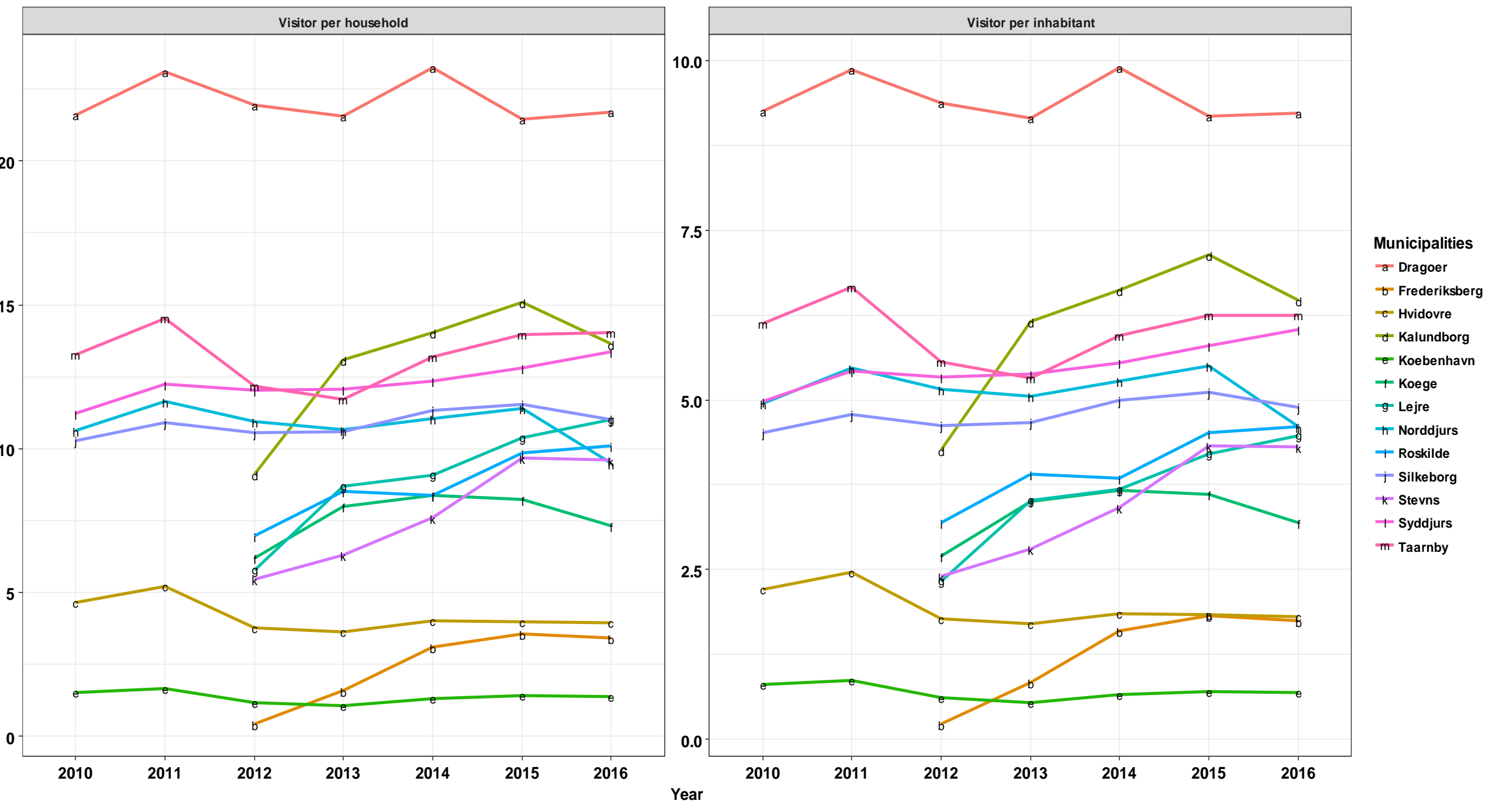


Figure S2: Graph showing the waste generation rates as function of number of visitors, households and person in municipalities

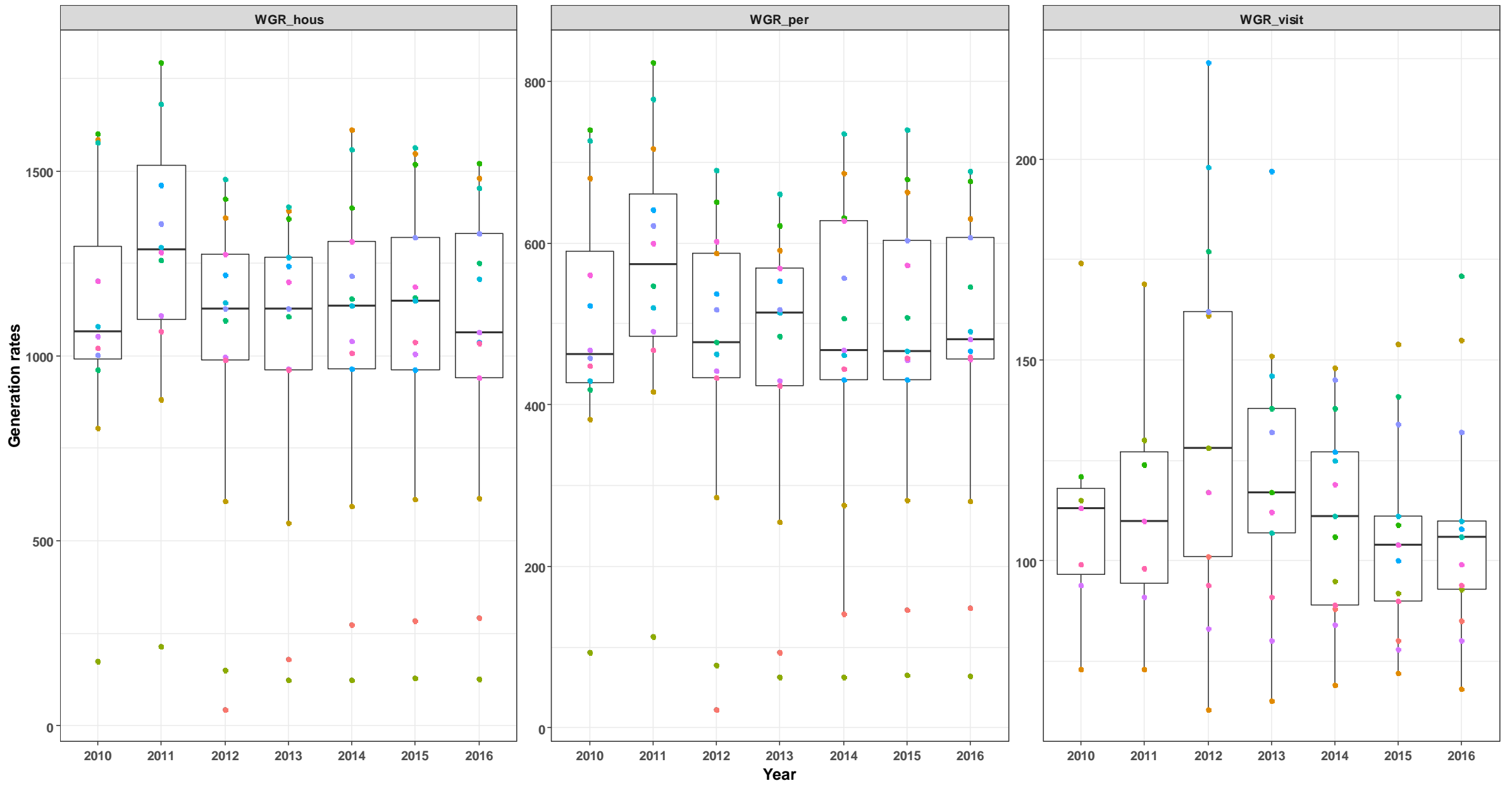

- Dragoer

Hvidovre

Koebenhavn

- Taarnby

Koege

- Kalundborg

Lejre

Stevns

Roskilde

- Syddjurs

- Norddjurs

Silkeborg 
Figure S3: Percentage of single-family houses and people living in single-family houses

Series wrc_2[, i]

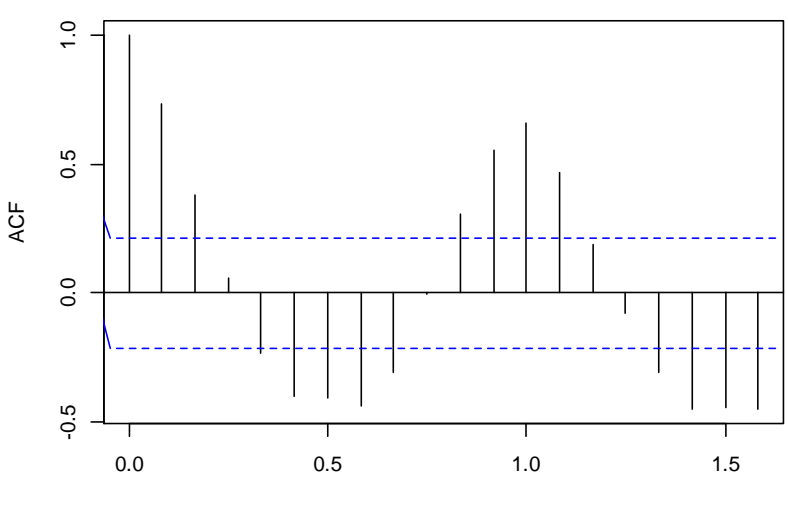

Lag

Series wrc_2[, i]

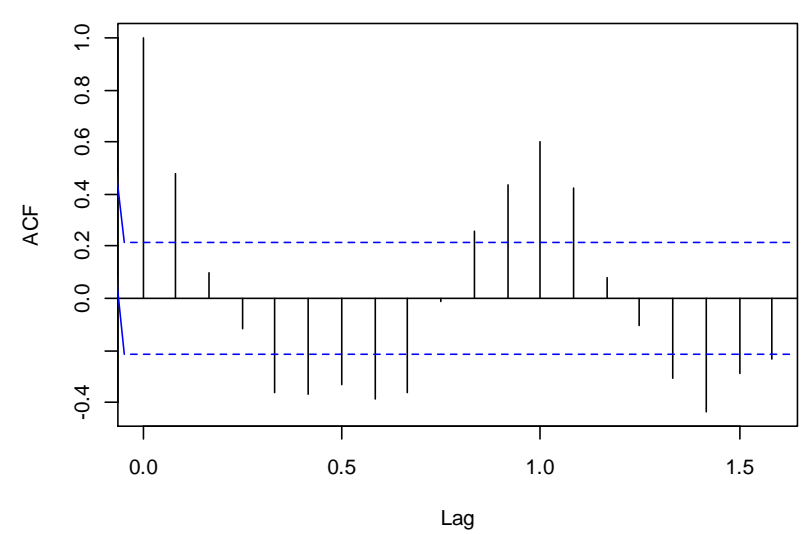

Series wrc_2[, i]

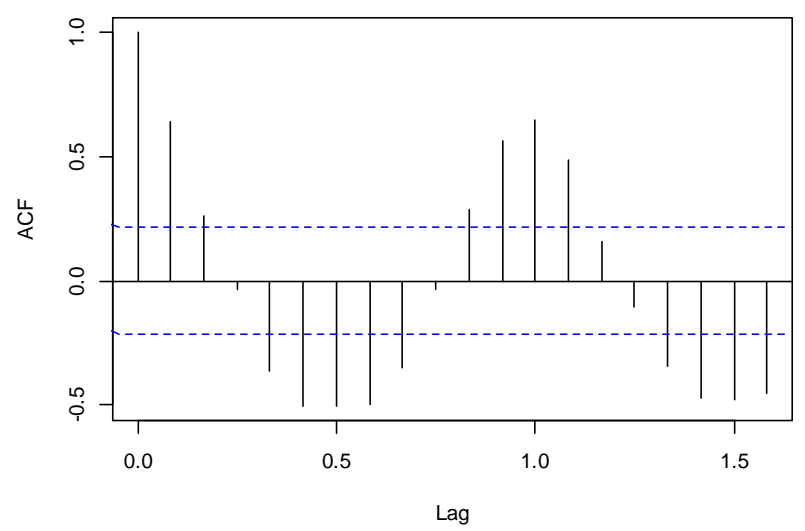

Series wrc_2[, i]

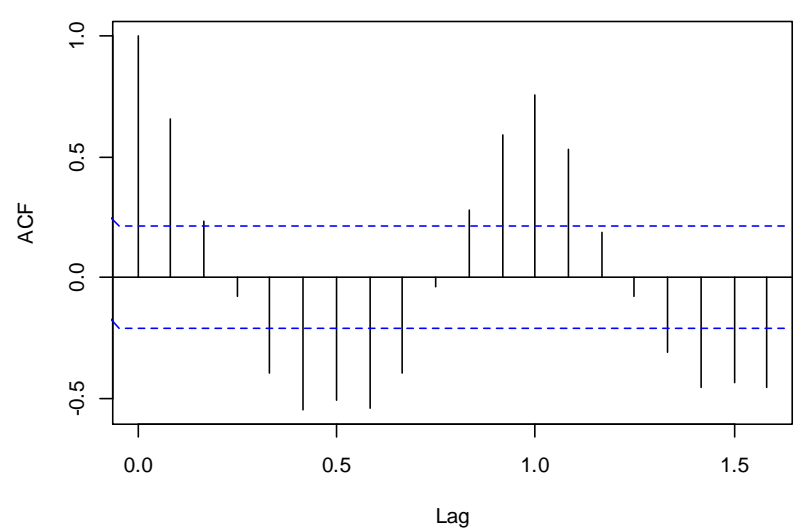




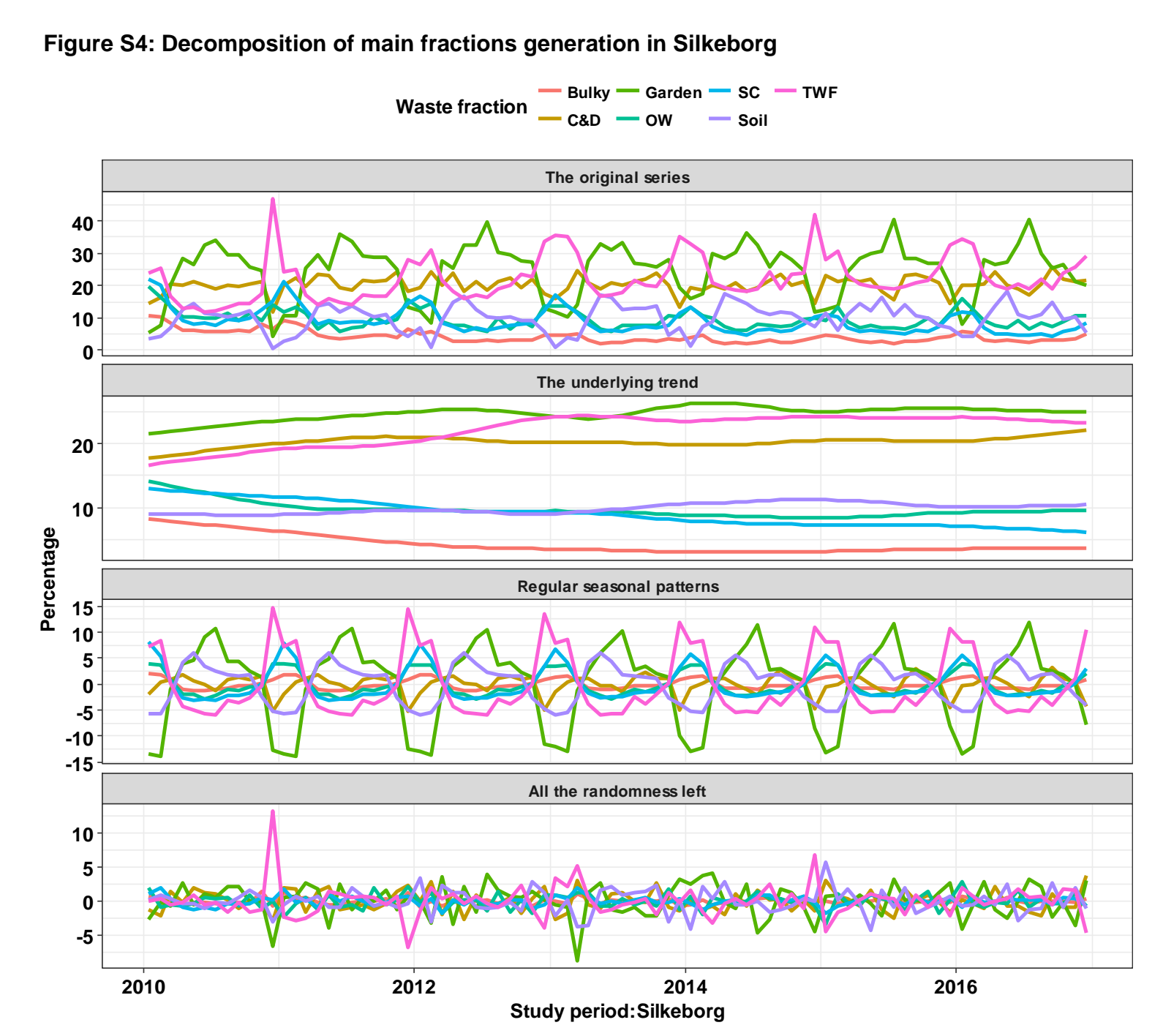


Figure S5: Decomposition of main fractions generation in Greater Copenhagen

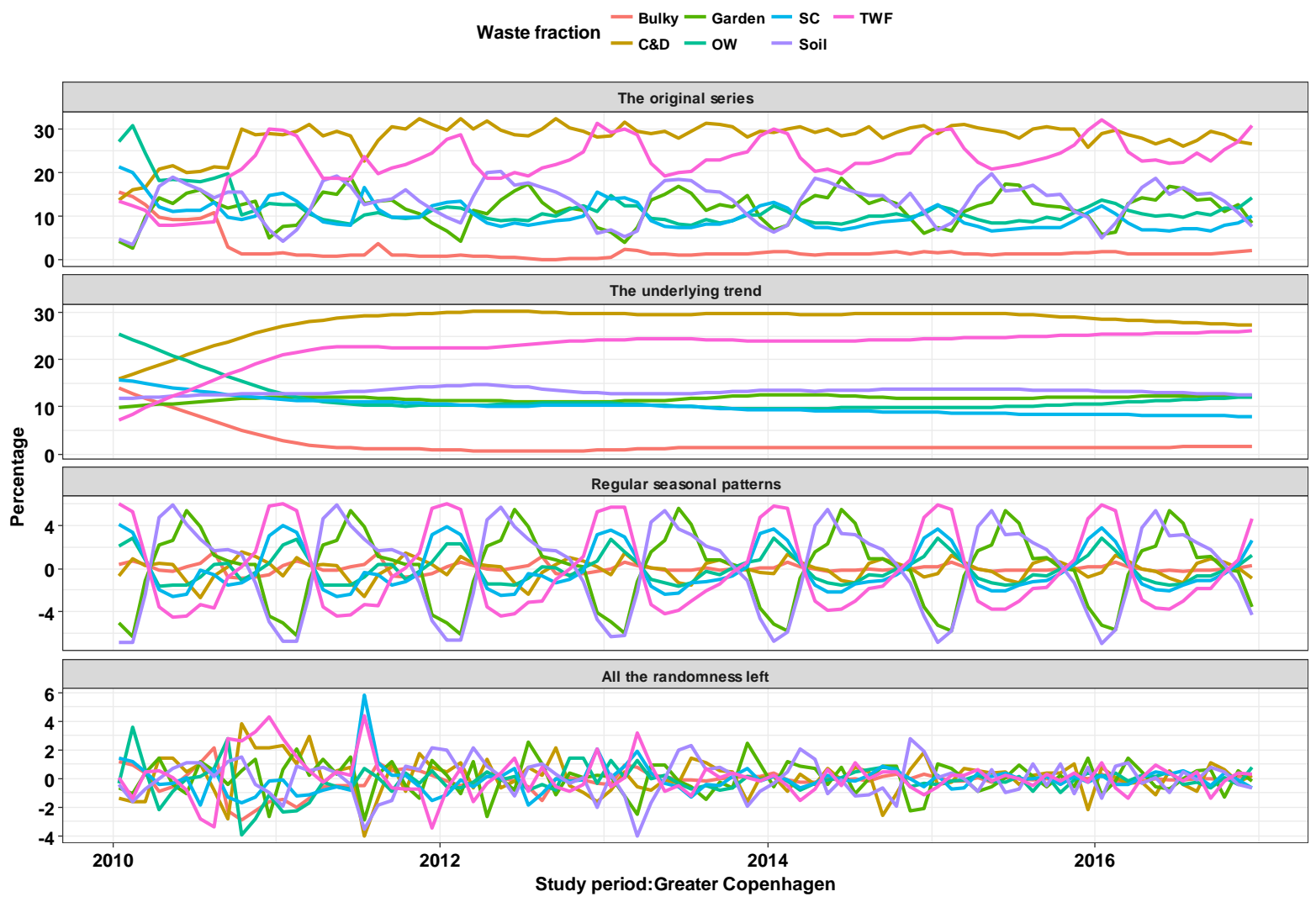


Figure S6: Decomposition of main fractions generation in Central Zealand

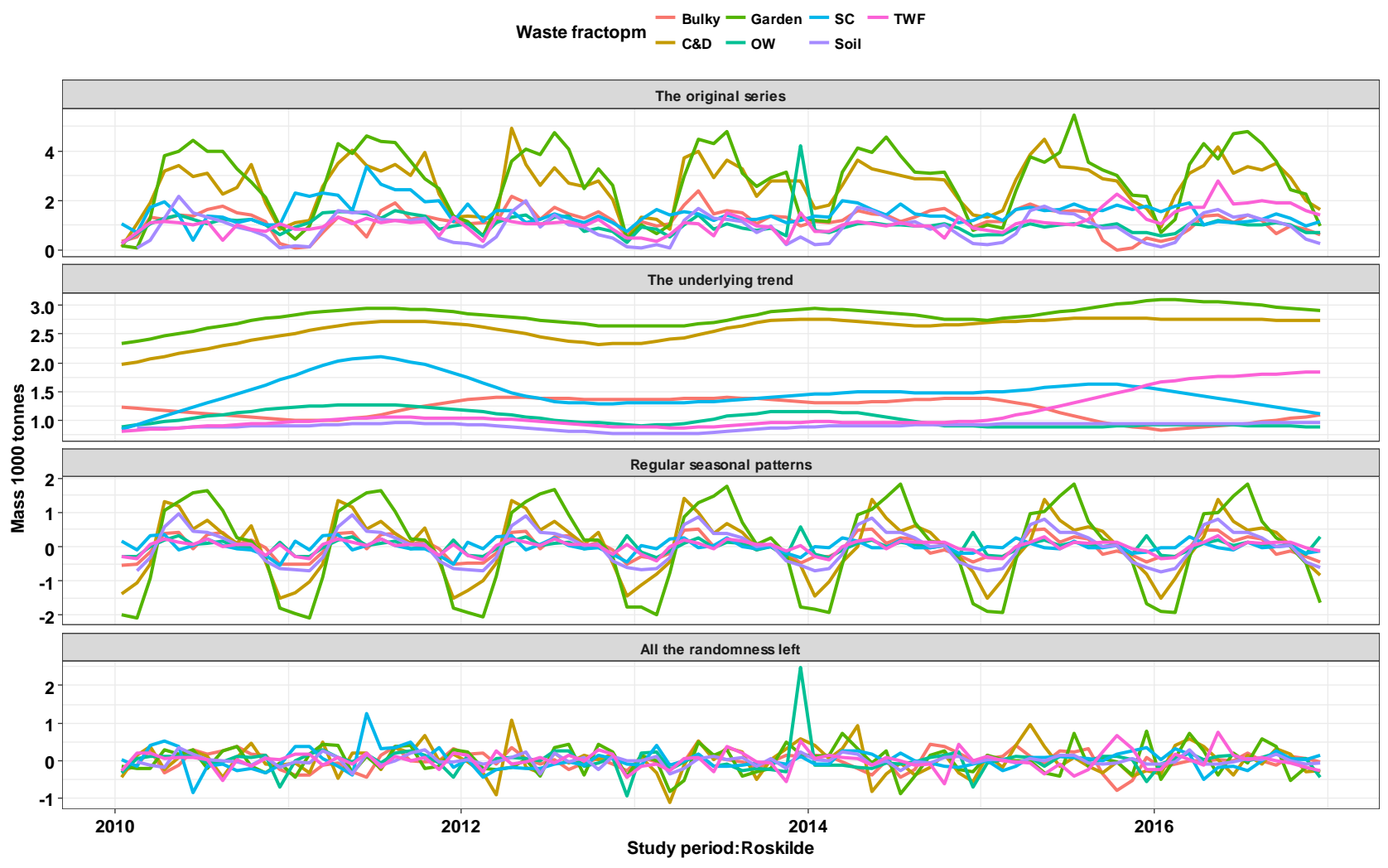


Figure S7: Decomposition of main fractions generation in Djursland

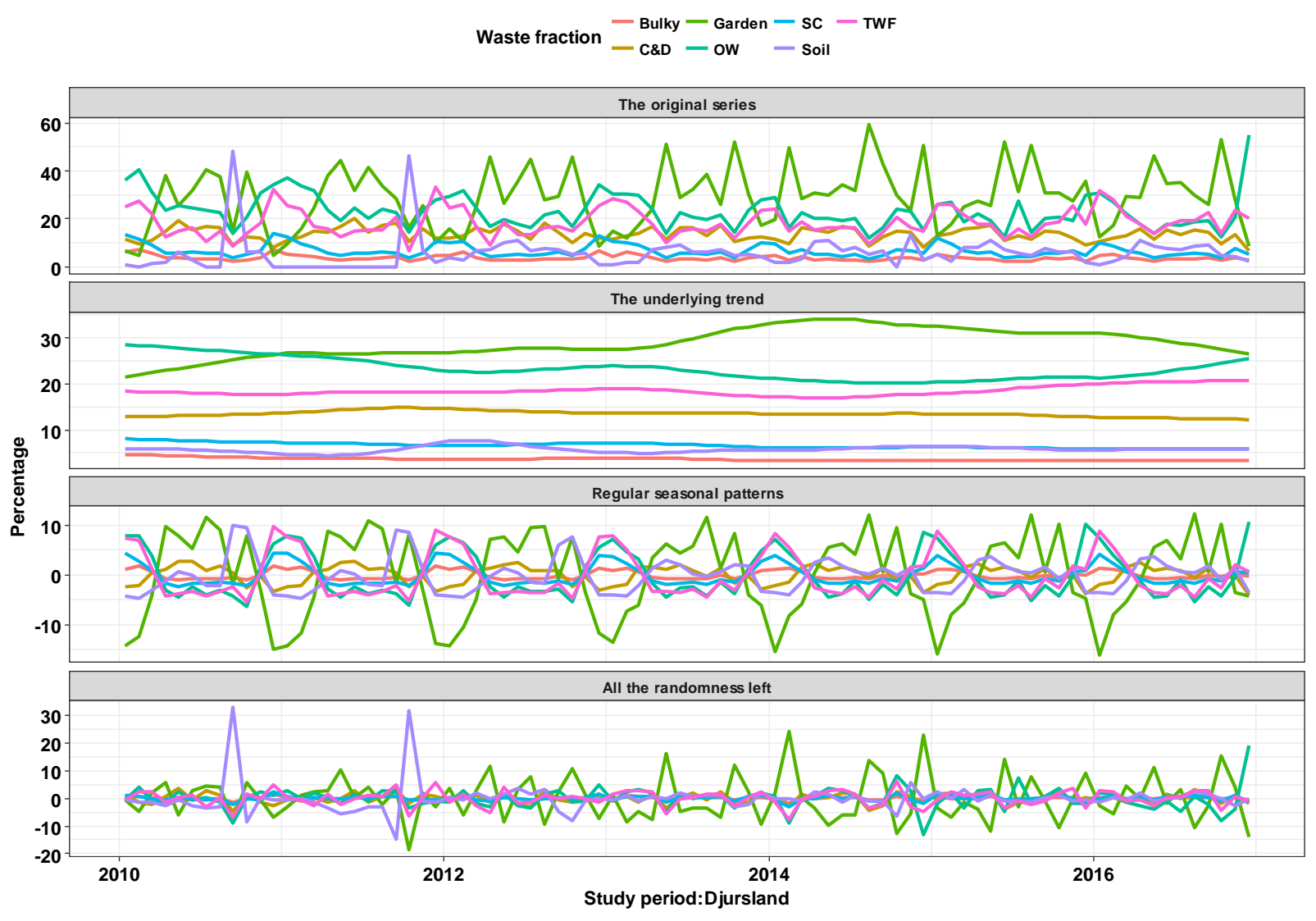


Figure S8: Plot from the multivariate Q-statistics and their $p$-values of aggregated waste generation in study areas. The dashed line denotes type I errors of $5 \%$.

p-values of Ljung-Box statistics

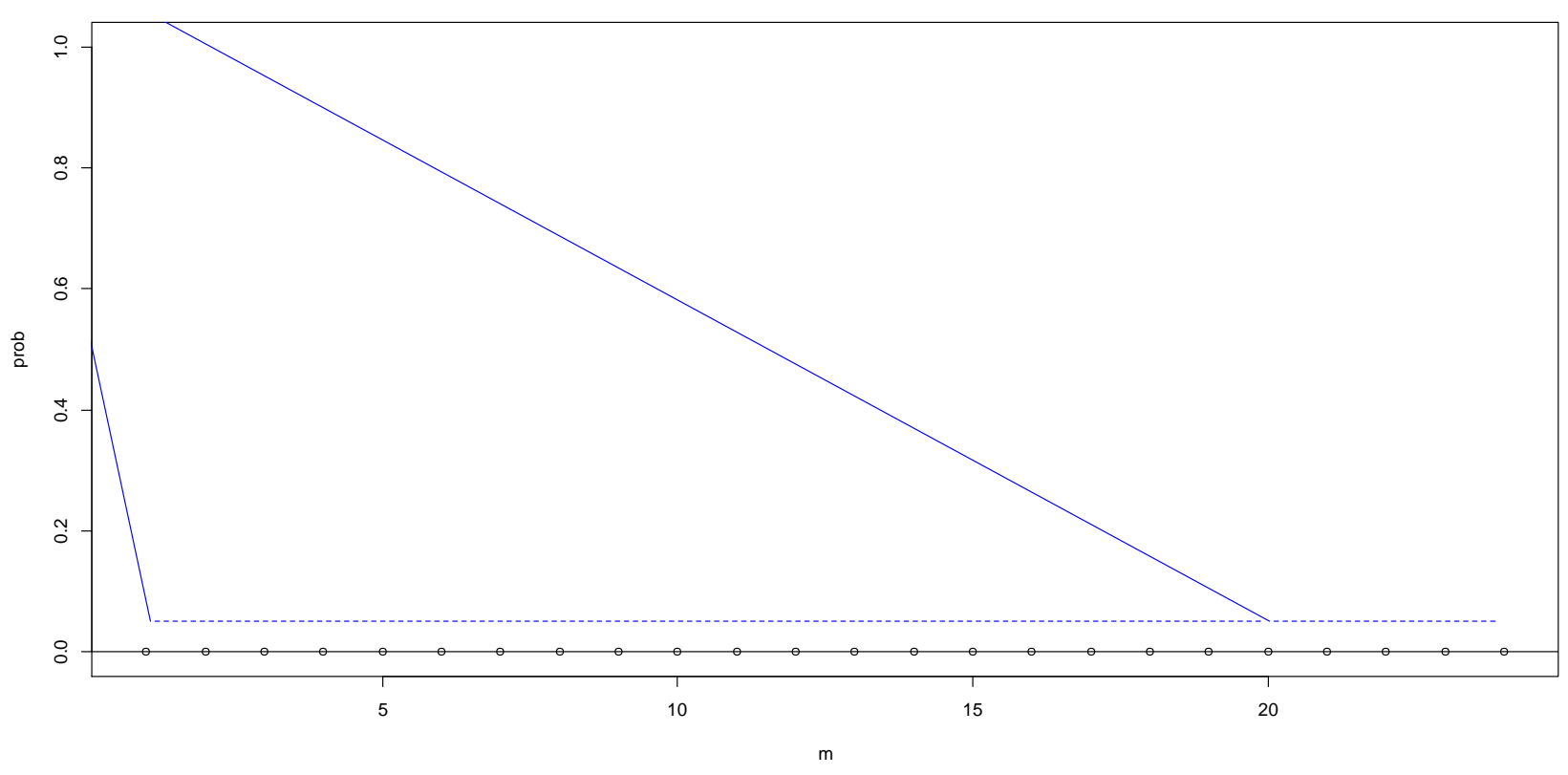


Figure S9: Seasonal generation of total waste at household waste recycling centers
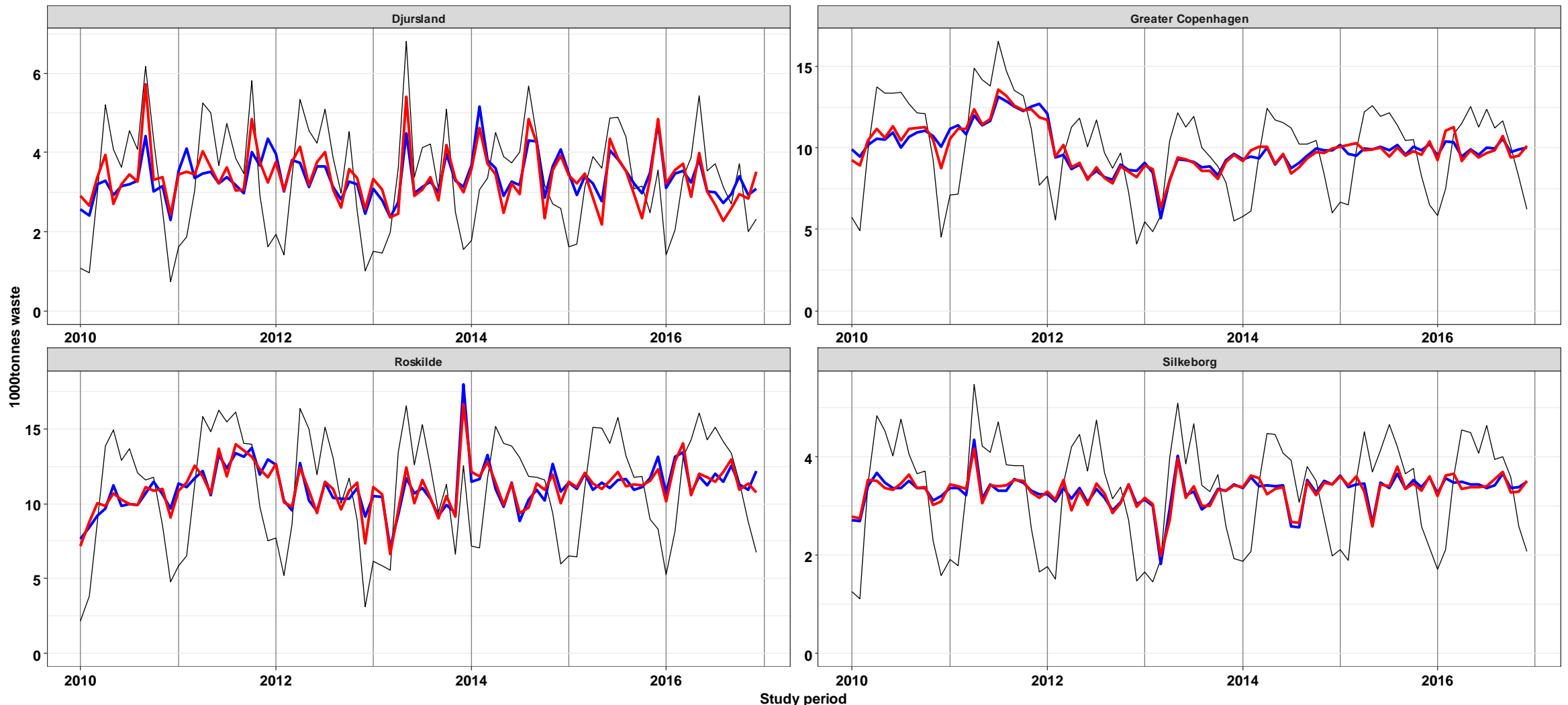
Figure S10: Seasonal generation of total waste at household waste recycling centers

- Bulky - Garden - SC - TWF

Waste fractions - C\&D - ow - soil

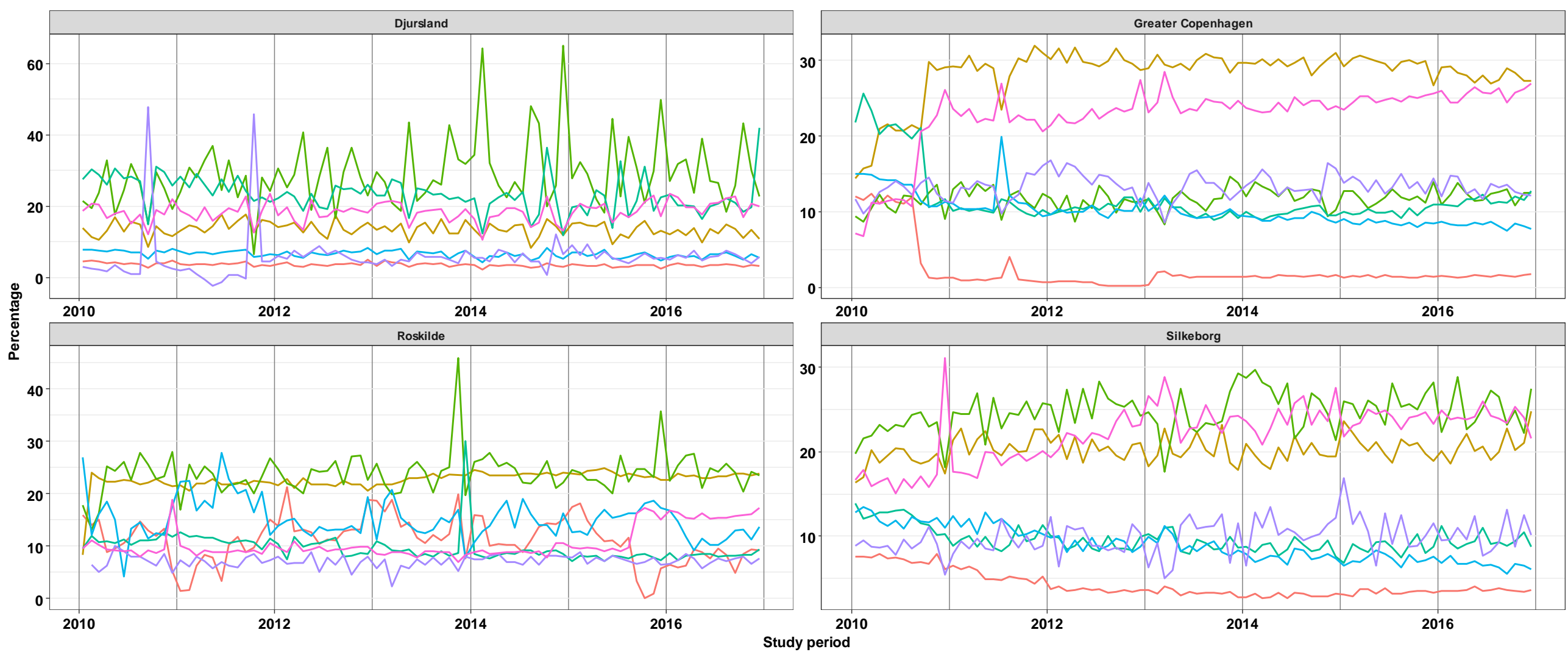


Figure S11: Waste generation cross-correlation plots for the period 2010-2016. The dashed lines indicate pointwise $95 \%$ confidence intervals
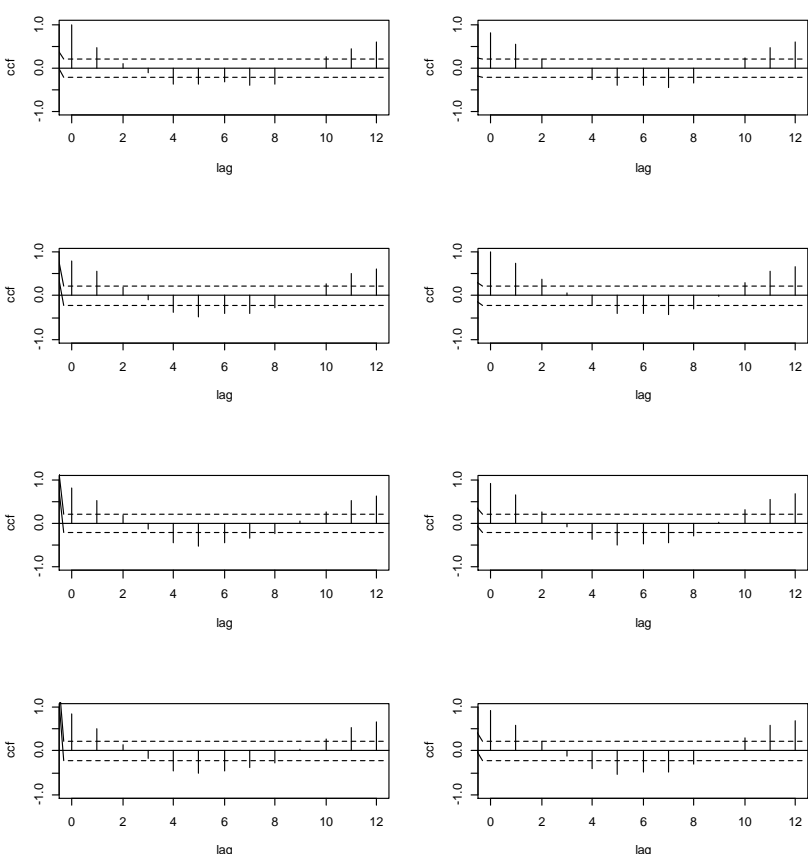
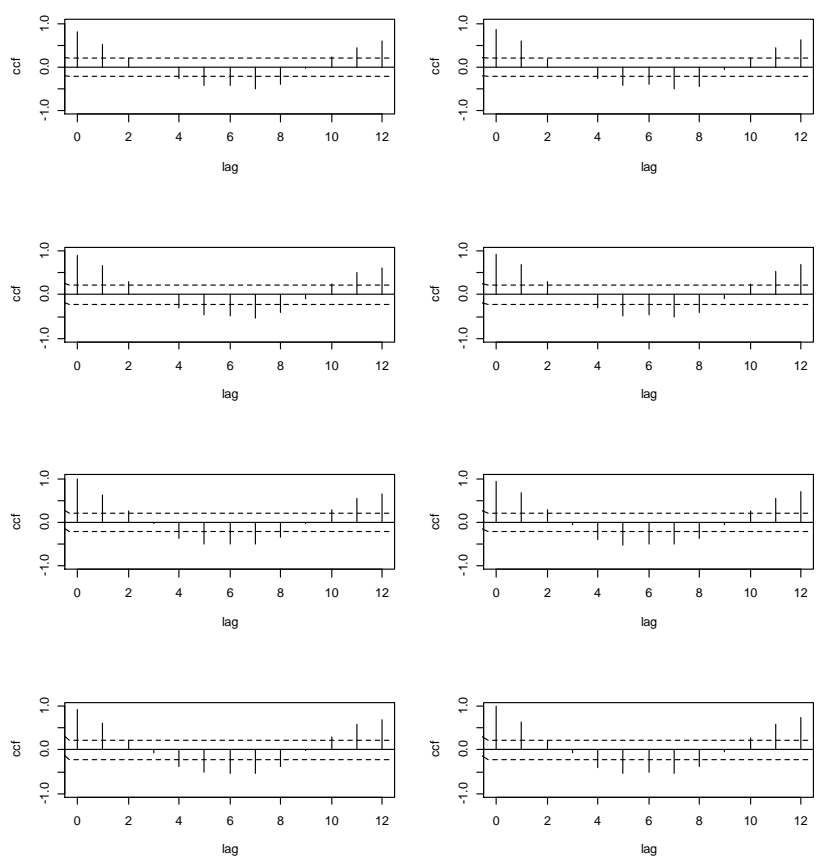
Figure S12: Waste treatment forms cross-correlation plots for the period 2010-2016. The dashed lines indicate pointwise $95 \%$ confidence intervals

Incineration

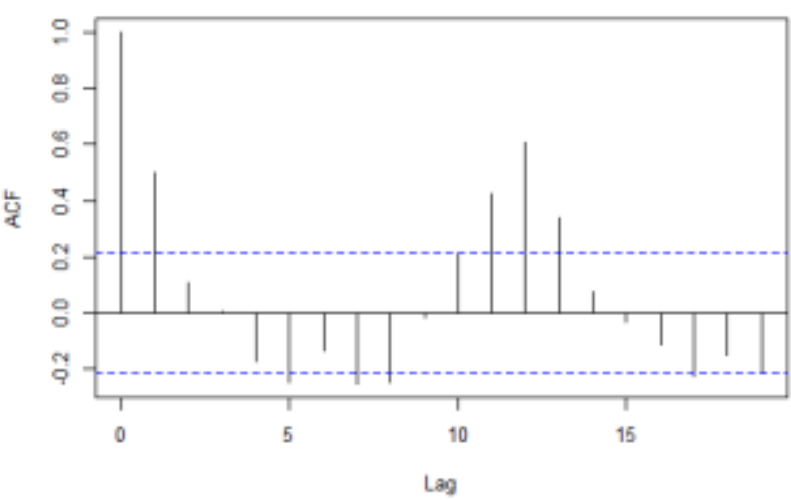

Recycling

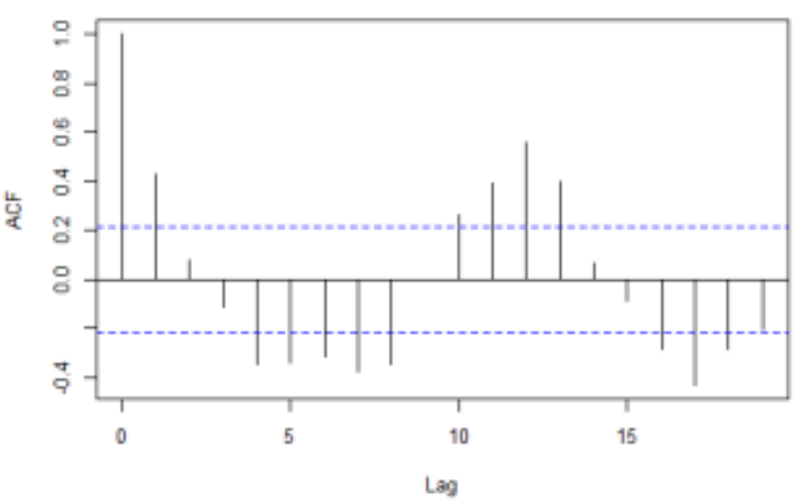

Other

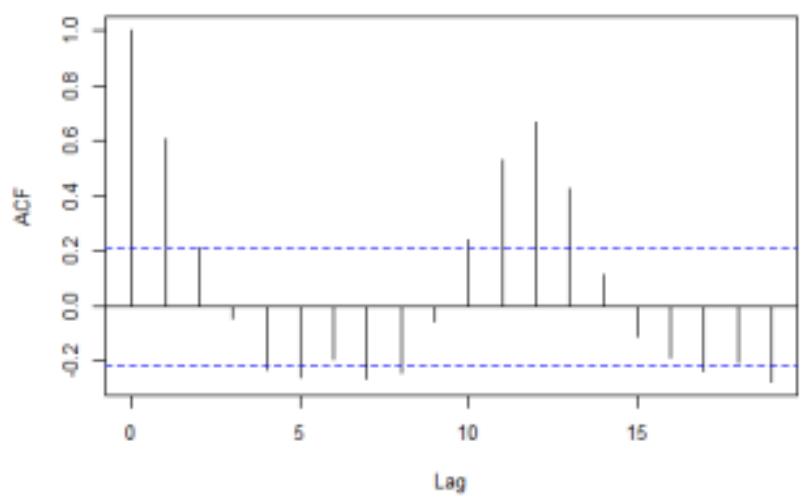


rm(list $=\mathrm{ls}()) \# \#$ Clear all history

"\%!in\%" <- function(x,table) match(x,table, nomatch = 0) == 0\#\#Function enabling

\#to exclude or omit multiple rows

\#Loading library

library(tidyverse)

library(lubridate)

library(forcats)

library(ggseas)

library(magrittr)

library(stringr)

library(stringi)

library(readxl)

library(mapDK)\#This package requires "devtools" \#

\#install.packages("devtools")

\#library(devtools)

\#devtools::install_github("sebastianbarfort/mapDK")

library(mapproj)

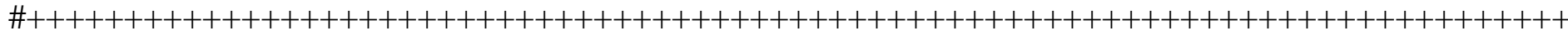

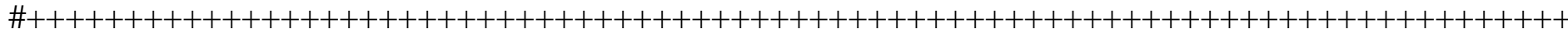

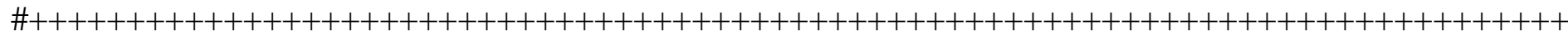

\#+++++++++++++++++++++++++++++++++++++++++++++++++++++++++++++++++++++++++++++++

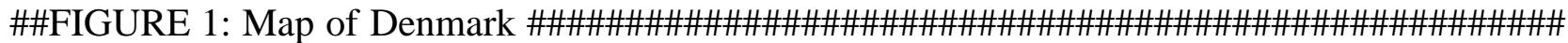

\#\#Loading data

\#Population from DST BOL103

DK_2018 <-read_excel("H:/Paper and Article/Paper_HRC/Arkive/data_all/DK_2018.xlsx", range = "B3:K696", col_types = c("text","numeric","text", "numeric", "numeric", "numeric", "numeric", "numeric",

"numeric", "numeric"))

\#Data cleaning and calculation to obtain the number of population and

\#inhabitants for all municipalities in Denmark

DK_population=

DK_2018\%>\%

mutate(Kommune=str_replace_all(Kommune, c("æ"="ae","å"="aа","ø"="oe","А̊"="Аa","Ø"="Oe","Æ"="Аe")))\%>\%

gather(Year,Pop, '2010`:'2016`)\%>\%

mutate(Pep=Pop*Antal_pers)\%>\%select(-Antal,-Antal_pers)\%>\%

group_by(Year,Kommune)\%>\%summarise(Household=sum(Pop),Person=sum(Pep))

\#\#Select only data for 2016

DK_population2016=

DK_population\%>\%

filter(Year\%in\% "2016")

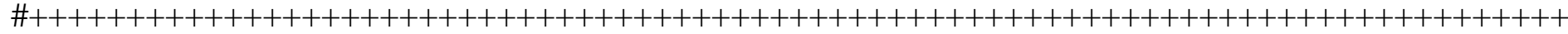
$\mathrm{Z}+++++++++++++++++++++++++++++++++++++++++++++++++++++++++++++++++++++++++++++++$ \#\#Loading data for geocode for all HWRC \#\#

geocode_2018 <-read_excel("H:/Paper and Article/Paper_HRC/Arkive/data_all/geocode_2018.xlsx", sheet $=$ "Sheet2")

\#\#\#Cleaning data process for geocode of HWRC \#geocode_2018\%>\%View()\# To view all dataset 
\#geocode_2018\%>\%head()\#to view the first 10 rows

\#\#Cleaning a data and calculations:

maps_data2=

geocode_2018\%>\%

mutate(Address=str_replace_all(Address,

c("æ"="ae","å"="aа","ø"="oe","Å"="Aa","Ø"="Oe","Æ"="Ae")))\%>\%

\#Replcace all danish letters with english letter

mutate( Returned address`=str_replace_all( Returned address`,

c("æ"="аe","å"="aа","Ø"="oe","Å"="Aa","Ø"="Oe","Æ"="Аe")))\%>\%

separate(Address, c("Street","Nummer","Country"),

sep $=$ ",", remove $=$ FALSE, convert $=$ FALSE) $\%>\%$

\#Replcace all danish letters with english letter

select(-Country,-`Returned address`)\%>\%

\#drop columns: countries and Returned address

distinct(Address,.keep_all = TRUE)\%>\%\#eleminate duplicate given address

distinct(Latitude,Longitude,.keep_all = TRUE)\%>\%\#eleminate duplicate for altitued

mutate(By=str_extract(Nummer, "[a-z,A-Z]+" ))\%>\%as.data.frame()\%>\%

\#extract only letter in the string (column)

mutate(Post=str_extract(Nummer, "[0-9]+" ))\%>\%as.data.frame()\%>\%

\#extract only number in the string (column)

distinct(By,Street,.keep_all = TRUE)\%>\%\#eleminate duplicate for city and street

filter(Address\%!in\% c("Borgervaenget 29, 2100 Koebenhavn Oe, Danmark",

"Graaskegaardevej 6, 8400 Ebeltoft, Danmark"))\%>\%\#exclude this HWRC

\#because of change in address. The new address is Sibeliusgade 80

mutate(New_area=ifelse(By\%in\%c("Kastrup","Hvidovre","Frederiksberg",

"Koebenhavn","Dragoer","Valby"),"Greater Copenhagen",

ifelse(By\%in\%c("Silkeborg","Them"),"Silkeborg",

ifelse(By\%in\%c("Eskebjerg","Kalundborg","Koege","Roskilde","Haarlev","Hoeng",

"Sejeroe","Store","Kirke","Bjaeverskov","Goerlev","Jyllinge"),"Central Zealand",

ifelse(By\%in\% c("Anholt","Allingaabro","Ebeltof","Roende",

"Glesborg","Grenaa","Hornslet","Knebel","Ryomgaard"),"Djursland",

"Not included")))))\%>\%

mutate(New_area=ifelse(Address\%in\%c("Rugvaenget 1, 4490 Jerslev Sj, Danmark",

"Vestergade 25B, 4130 Viby Sjaelland",

"Brohaven 2, Jyllinge, Danmark"),

"Central Zealand",paste0(New_area)))\%>\%

mutate(sorting=ifelse(Address \%in\%

c("Tandskovvej 17, 8600 Silkeborg, Danmark",

"Tietgensvej 7, 8600 Silkeborg, Danmark",

"Herjedalgade 2-4, 2300 Koebenhavn S, Danmark",

"Avedoereholmen 97, 2650 Hvidovre, Danmark",

"Kalorievej 2, 8500 Grenaa, Danmark",

"Vestergade 25B, 4130 Viby Sjaelland",

"Brohaven 2, Jyllinge, Danmark"),"WS","Not"))

\#end of the first data cleaning

unique(maps_data2\$New_area)

\#\#Forced the order of variables

maps_data2\$New_area=factor(maps_data2\$New_area,levels $=\mathrm{c}($

"Greater Copenhagen","Central Zealand","Djursland","Silkeborg","Not included"))

$\mathrm{\#}++++++++++++++++++++++++++++++++++++++++++++++++++++++++++++++++++++++++++++$

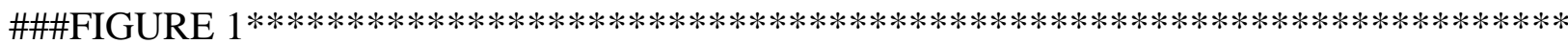

mapDK(values = "Person", id = "Kommune", data = DK_population2016)+

geom_point(data =maps_data2, 
aes(Longitude,Latitude,group=New_area,

colour=New_area,shape=sorting),size=3,fill="black",

alpha $=\mathrm{I}(0.7))+$

scale_shape_manual $($ values $=c(20,17))+$

scale_color_manual(values=c("red","green","magenta","blue","grey"))+

theme(axis.text = element_text("'))+

theme_bw ()$^{+}$

labs(x="Longitude",y="Latitude",colour="Study areas",

group="Number of inhabitant per municipality")+

theme(text =element_text $($ colour = "black", face = "bold", size = 12) $)$

\#End of Figure 1

\#\%\%\%\%\%\%\%\%\%\%\%\%\%\%\%\%\%\%\%\%\%\%\%\%\%\%\%\%\%\%\%\%\%\%\%\%\%\%\%\%\%\%\%\%\%\%\%\%\%\%\%\%\%\%\%\%\%

\%\%\%\%\%\%\%\%\%\%\%\%\%\%\%\%\%\%\%\%\%\%

\#+++++++++++++++++++++++++++++++++++++++++++++++++++++++++++++++++++++++++++++++

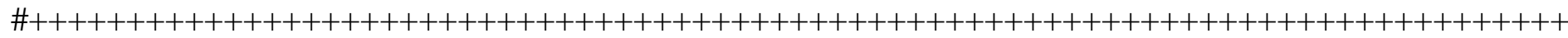

\#+++++++++++++++++++++++++++++++++++++++++++++++++++++++++++++++++++++++++++++++

\#\#Loading data

wrc2018_v8= read.table("H:/Paper and Article/Paper_HRC/Arkive/data_all/wrc2018_v8.txt")

wrc2018_v8=wrc2018_v8\%>\%

mutate(date1=ymd( paste(Year, Months, "15", sep="-")))\%>\%

mutate(Kommune=fct_recode(Kommune,

"Koebenhavn"="Copenhagen"))\#\%

\#create a new column of type date

wrc2018_v8\$Months=factor(wrc2018_v8\$Months,levels=c("Jan","Feb","Mar","Apr","Maj"

,"Jun","Jul","Aug","Sep","Okt","Nov","Dec"))

\#Arrange the order of months

month=c("Jan","Feb","Mar","Apr","Maj",

"Jun","Jul","Aug","Sep","Okt","Nov","Dec")

wrc2018_v8\%>\%View()

\#\#Order of municipalities

muni=c("Frederiksberg","Dragoer","Hvidovre",

"Koebenhavn","Taarnby","Koege","Kalundborg","Lejre",

"Stevns","Roskilde","Syddjurs","Norddjurs","Silkeborg")

\#\#Table 2: Total waste per year per recycling centre\#****************************

Table2 $=$

wrc2018_v8\%>\%

group_by(Year,Affaldsselskab,Kommune,Genbrugspladser)\%>\%

summarise(Tot=sum(Maengde) $\%>\%$

spread(Year,Tot,fill $=0) \%>\%$

ungroup ()$\%>\% \#$ ungroup grouping factor

mutate $($ Kommune=factor $($ Kommune,levels $=$ muni $)) \%>\%$

\#set a new order of municipalities

arrange(Kommune)\#show Kommune with desired order

\#+++

Table2_update $=$

wrc2018_v8\%>\%

group_by(Year,Affaldsselskab)\%>\%

summarise(Tot $=$ sum(Maengde) $) \%>\%$

spread(Year,Tot,fill $=0) \%>\%$

mutate(Affaldsselskab=factor(Affaldsselskab, 
levels = c("Greater Copenhagen","Central Zealand","Djursland","Silkeborg")))\#show Kommune with desir

Table2_update\%>\%View()

\#+++++

\#\#Export Table 2 in Excel file

library(writexl)

write_xlsx(Table2, "H:/Paper and Article/Paper_HRC/Results/2018-05-Results/Table2.xlsx")

\#End of Table 2

\#\%\%\%\%\%\%\%\%\%\%\%\%\%\%\%\%\%\%\%\%\%\%\%\%\%\%\%\%\%\%\%\%\%\%\%\%\%\%\%\%\%\%\%\%\%\%\%\%\%\%\%\%\%\%\%\%\% \%\%\%\%\%\%\%\%\%\%\%\%\%\%\%\%\%\%\%\%\%\%

\#\%\%\%\%\%\%\%\%\%\%\%\%\%\%\%\%\%\%\%\%\%\%\%\%\%\%\%\%\%\%\%\%\%\%\%\%\%\%\%\%\%\%\%\%\%\%\%\%\%\%\%\%\%\%\%\%\% \%\%\%\%\%\%\%\%\%\%\%\%\%\%\%\%\%\%\%\%\%\%

\#+++++++++++++++++++++++++++++++++++++++++++++++++++++++++++++++++++++++++++++++

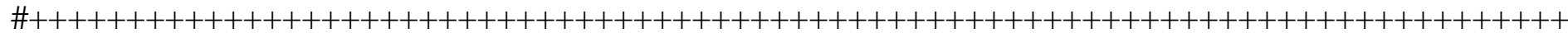

\#\#Figure 2

\#\#This code generate a table which can be used to make Figure 2 in Excel

wrc2018_v8\%>\%

group_by(Year,Affaldsselskab)\% $>\%$

summarise(Tot=sum(Maengde)/1000)\%>\%\#1000Mg

mutate(Affaldsselskab=fct_recode(Affaldsselskab,

"Central Zealand"="Roskilde"))\%>\%

ungroup()\%>\%\#ungroup grouping factor

mutate(Affaldsselskab=factor(Affaldsselskab,

levels = c("Greater Copenhagen","Central Zealand","Djursland","Silkeborg")))\%>\%

spread(Affaldsselskab,Tot,fill = 0)\%>\%iew()\#show Kommune with desired order

\#\#End of table enabling to build Figure 2

\#\%\%\%\%\%\%\%\%\%\%\%\%\%\%\%\%\%\%\%\%\%\%\%\%\%\%\%\%\%\%\%\%\%\%\%\%\%\%\%\%\%\%\%\%\%\%\%\%\%\%\%\%\%\%\%\%\% \%\%\%\%\%\%\%\%\%\%\%\%\%\%\%\%\%\%\%\%\%\%

\#\%\%\%\%\%\%\%\%\%\%\%\%\%\%\%\%\%\%\%\%\%\%\%\%\%\%\%\%\%\%\%\%\%\%\%\%\%\%\%\%\%\%\%\%\%\%\%\%\%\%\%\%\%\%\%\%\% \%\%\%\%\%\%\%\%\%\%\%\%\%\%\%\%\%\%\%\%\%\%

\#++++++++++++++++++++++++++++++++++++++++++++++++++++++++++++++++++++++++++++++

\#Table 3

\#\#Aggregated (yearly) Data per year for visitors, inhabitants and area

\#Visitors and mass of waste for Greater Copenhagen, RenoDjurs and Silkeborg

para2 <- read.csv("H:/Paper and Article/Paper_HRC/arkive/data_all/para2.txt", sep="')

\#\#Loading monthly waste data from Central Zealand and Silkeborg

visit_v1 <-read_excel("H:/Paper and Article/Paper_HRC/Arkive/data_all/visit_v1.xlsx")

visit_v1=visit_v1\%>\% mutate(date1=ymd( paste(Year, Months, "15", sep="-")))\#\%>

visit_v1\$Months=factor(visit_v1\$Months,levels=c("Jan","Feb","Mar","Apr","Maj"

,"Jun","Jul","Aug","Sep","Okt","Nov","Dec"))

\#+++++++++++++++++++++++++++++++++++++++++++++++++++++++++++++++++++++++++++++++

\#\#data for housing type distribution:

\#\#Loading number of person and housing type for all Denmark from 2010-2017

DK_houses <-read_excel("H:/Paper and Article/Paper_HRC/Arkive/data_all/DK_houses.xlsx", range $=$ "B3:L300")

DK_houses\% $>\%$ head()

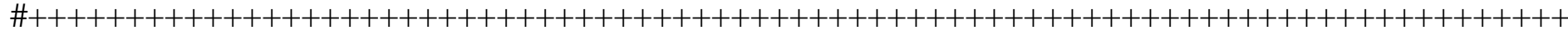

\#Import data for areal of municipalities from 2010 to 2016

\#http://www.statistikbanken.dk/statbank5a/selectvarval/saveselections.asp

library(stringr)

DK_areal <- read_excel("H:/Paper and Article/Paper_HRC/Arkive/data_all/DK_areal.xlsx", range = "A3:J102", col_types = c("text", 
"numeric", "numeric", "numeric",

"numeric", "numeric", "numeric",

"numeric", "numeric", "numeric"))

\#\#Cleaning data

Dk_area $=$

DK_areal\%>\%

mutate $($ Kommune $=$

str_replace_all(Name,\#Find all danish characters and replace them with

\#The tricky part is to remember to have mutate at the beginning

\#For more inf. page 215, $\mathrm{R}$ for Data Science

c("æ"="ae","å"="aa","ø"="oe","Å"="Аa","Ø"="Oe","Æ"="Аe")))\%>\%

gather(Year,area_km2, ’2008`:2016”)

\#\#Table containing data for Silkeborg and central zealand

table3_1=

wrc2018_v8\%>\%

mutate(Year=as.character(Year) $) \%>\%$

filter(Affaldsselskab\%in\% c("Roskilde","Silkeborg"))\%>\%

mutate(Affaldsselskab=fct_recode(Affaldsselskab,

"Central Zealand"="Roskilde"))\%>\%

group_by(Year,Affaldsselskab,Kommune)\%>\%

summarise(Tot=sum(Maengde) $) \% \%$

\#calculate mass

left_join(Dk_area[,-1],by = c("Year", "Kommune"))\%>\%

\#add areal for each municipality

\#Afterwards, we added a table cointing number of visitors

left_join(visit_v1\%>\%

filter(Affaldsselskab\%in\% c("Central Zealand","Silkeborg"))\%>\%

group_by(Year,Affaldsselskab,Kommune)\%>\%

summarise(Visitors=sum(Visit))) $\%>\%$

\#The next step is to add data of inhabitant and number of houses

left_join(DK_population)

\#\#show the first 10 lines of the results

table3_1\%>\%head()

\#\#Table containing data for Greater Copenhagen and Djursland

table3_2=

wrc2018_v8\%>\%

mutate(Year=as.character(Year))\%>\%

filter(Affaldsselskab\%!in\% c("Roskilde","Silkeborg"))\%>\%

group_by(Year,Affaldsselskab,Kommune)\%>\%

summarise(Tot=sum(Maengde) $\%>\%$

\#calculate mass

left_join(Dk_area[,-1],by = c("Year", "Kommune"))\%>\%

\#add areal for each municipality

\#Afterwards, we added a table (para2) cointing number of visitors,inhabitant

\#total mass per year, number of household

left_join(para2\%>\%

mutate(Year=as.character(Year) $) \%>\%$

filter(Affaldsselskab\%!in\% c("Silkeborg"))\%>\%

mutate(Affaldsselskab=fct_recode(Affaldsselskab,

"Greater Copenhagen"="ARC", 
"Djursland"="RenoDjurs"))\%>\%

select(-Areal_km2))

\#++++++++++++++++++++++++++++++++++++++++++++++++++++++++++++++++++++++++++++++++

\#\#\#show the first 10 lines of the results

table3_2_1<-

table3_2\%>\%View()

group_by(Year,Affaldsselskab)\%>\%

summarise(Tot=sum(Tot),

area_km2=sum(area_km2),

Maengde $=$ sum(Maengde),

Visitors $=$ sum(Visitors),

Household=sum(Household),

Person=sum(Person))

\#++++++++++++++++++++++++++++++++++++++++++++++++++++++++++++++++++++++++++++++

\#Combine all data

Table3=

table3_2_1\%>\%

bind_cols(Maengde $=$ rep $(0$, nrow $($ table3_2_1) $)) \%>\%$

bind_rows(table3_2_1)\% $>\%$

mutate(WGR_hous=(Tot/Household)*1000,

WGR_per $=($ Tot $/$ Person $) * 1000$,

WGR_visit $=($ Tot/Visitors $) * 1000) \%>\%$

select(Year,Affaldsselskab,WGR_hous,WGR_per,WGR_visit)\%>\%

gather(Parameters, Values,WGR_hous:WGR_visit)\%>\%View()

tidyr::spread(Year,Values)\%>\%ungroup()\%>\%

mutate(Affaldsselskab=factor(Affaldsselskab,

levels = c("Greater Copenhagen","Central Zealand","Djursland","Silkeborg")))\%>\%

arrange(Affaldsselskab,Kommune)\%>\%

mutate_if(is.numeric, funs(round(., 0)))\#\#Rounding numbers to zero

\#+++++++++++++++++++++++++++++++++++++++++++++++++++++++++++++++++++++++++++++++

table3_2_update $=$

wrc2018_v8\%>\%

mutate(Year=as.character(Year))\%>\%

filter(Affaldsselskab\%!in\% c("Roskilde","Silkeborg"))\%>\%

group_by(Year,Affaldsselskab)\%>\%

summarise(Tot=sum(Maengde) $\%>\%$

\#calculate mass

left_join(Dk_area[,-1],by = c("Year"))\%>\%

\#add areal for each municipality

\#Afterwards, we added a table (para2) cointing number of visitors,inhabitant

\#total mass per year, number of household

left_join(para2\%>\%

mutate(Year=as.character(Year) $) \%>\%$

filter(Affaldsselskab\%!in\% c("Silkeborg"))\%>\%

mutate(Affaldsselskab=fct_recode(Affaldsselskab,

"Greater Copenhagen"="ARC",

"Djursland"="RenoDjurs"))\%>\%

select(-Areal_km2))

Table3_update $=$

table3_1\%>\%

bind_cols(Maengde $=$ rep $(0$, nrow(table3_1) $)) \%>\%$

bind_rows(table3_2)\%>\% 


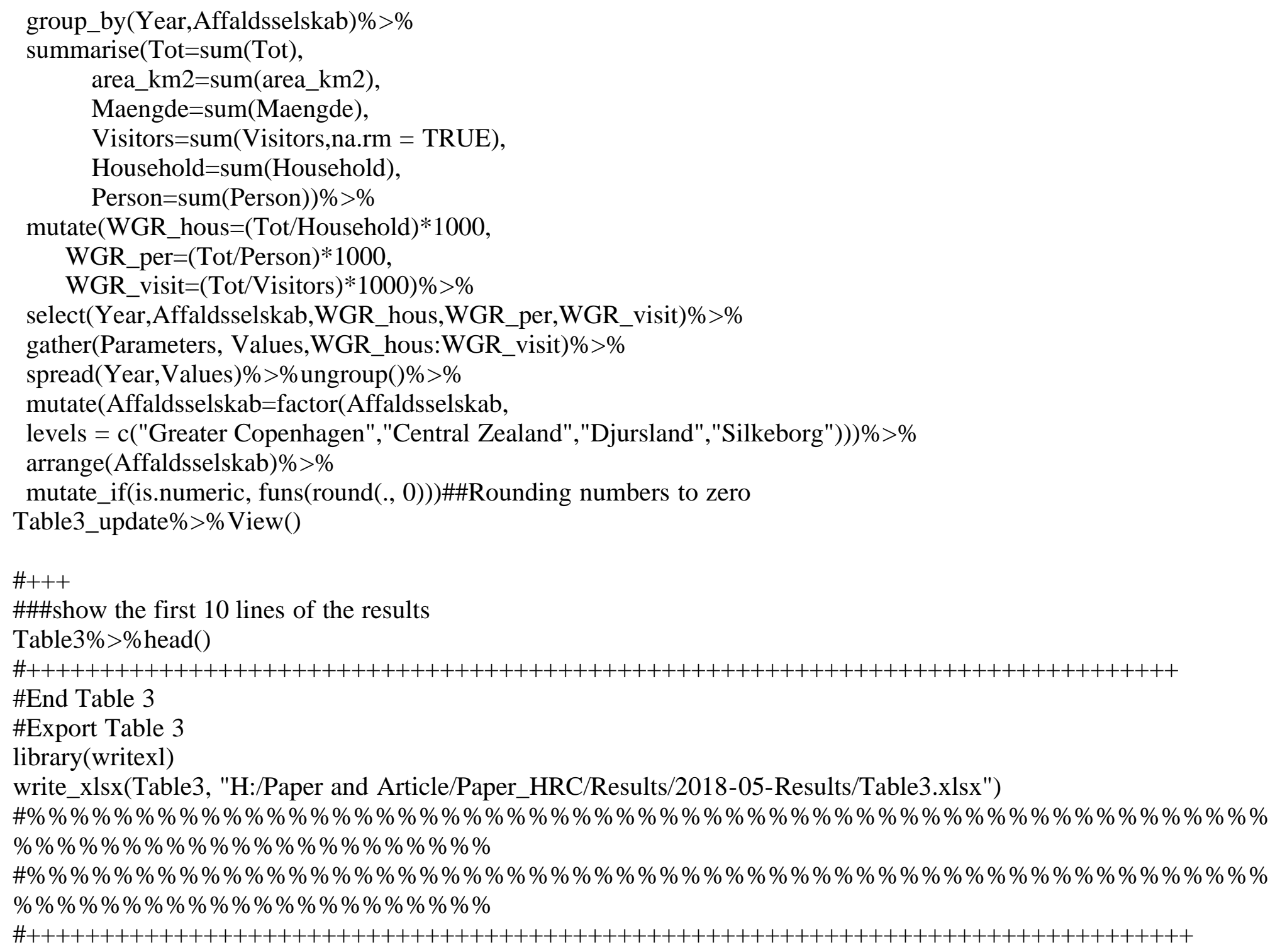




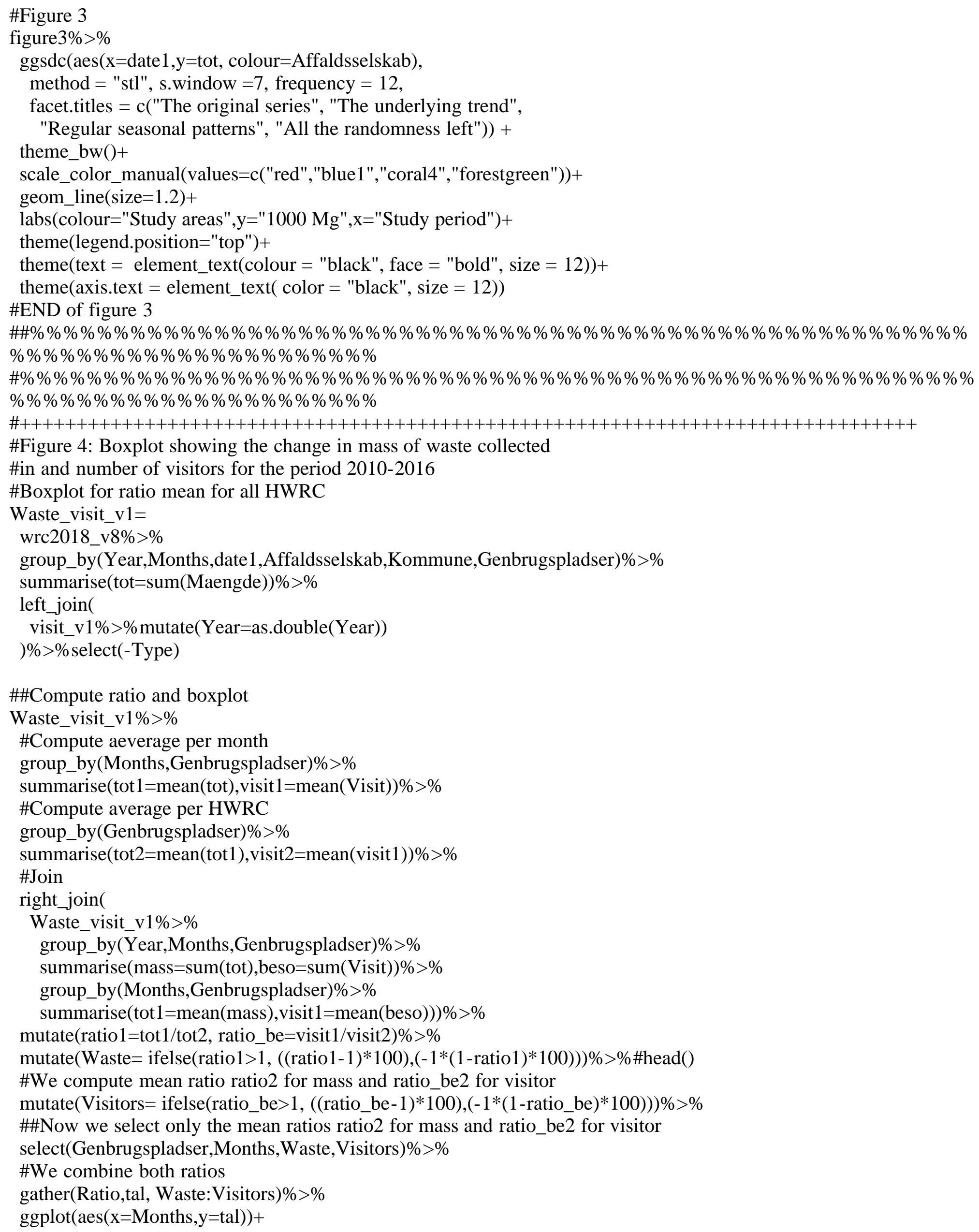


geom_boxplot(aes(colour=Ratio),position=position_dodge(.9))+

stat_summary(fun.y=mean, colour="darkred", geom="point",

aes(group=Ratio), position=position_dodge(.8),

shape $=18$, size $=3$, show_guide $=$ FALSE $)+$

geom_hline $($ yintercept $=0$, colour="red",size $=1)+$

theme_bw()+

theme(legend.position="top")+

labs(x="Months", $\mathrm{y}=$ "Mean ratio in \%")+

theme(text =element_text(colour = "black", face = "bold", size =12))

\#END of figure 4

$\mathrm{\# ++++++++++++++++++TOOOOOOO} \mathrm{BE}$

CHECKED +++++++++++++++++++++++++++++++++++++++++++++++

\#\#\%\%\%\%\%\%\%\%\%\%\%\%\%\%\%\%\%\%\%\%\%\%\%\%\%\%\%\%\%\%\%\%\%\%\%\%\%\%\%\%\%\%\%\%\%\%\%\%\%\%\%\%\%\%\%\% $\% \% \% \% \% \% \% \% \% \% \% \% \% \% \% \% \% \% \%$

\#\%\%\%\%\%\%\%\%\%\%\%\%\%\%\%\%\%\%\%\%\%\%\%\%\%\%\%\%\%\%\%\%\%\%\%\%\%\%\%\%\%\%\%\%\%\%\%\%\%\%\%\%\%\%\%\%\% $\% \% \% \% \% \% \% \% \% \% \% \% \% \% \% \% \% \%$

\#+++++++++++++++++++++++++++++++++++++++++++++++++++++++++++++++++++++++++++++++

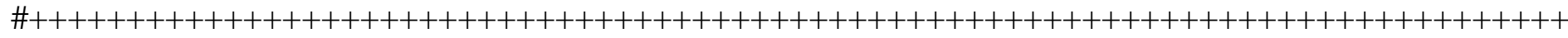

\#\#Figure 8

install.packages("ggtern")

library(ggtern)

figure8=

wrc2018_v8\%>\%

mutate(Affaldsselskab=fct_recode(Affaldsselskab,

"Central Zealand"="Roskilde"))\%>\%

group_by(Year,Affaldsselskab,Genbrugspladser,Treatment)\%>\%\#head()

summarise(Tot=sum(Maengde)/1000)\%>\%\#head()

group_by(Year,Affaldsselskab,Genbrugspladser) \%>\%

transmute(Treatment, per_waste $=($ Tot $/ \operatorname{sum}($ Tot $) * 100)) \%>\%$

spread(Treatment, per_waste, fill $=0$ )

\#filter(Affaldsselskab \%in\% c("Greater Copenhagen"))\%>\%

fig8a $=$

wrc2018_v8\%>\%

mutate(Affaldsselskab=fct_recode(Affaldsselskab,

"Central Zealand"="Roskilde"))\%>\%

group_by(Year,Affaldsselskab,Genbrugspladser,Treatment)\%>\%\#head()

summarise(Tot=sum(Maengde)/1000)

ternaryplot(figure8[,c("Incineration","Other","Recycling")])

ggtern(data=figure8, aes( $\mathrm{y}=$ Incineration, $\mathrm{x}=$ Other, $\mathrm{z}=$ Recycling $))+$

geom_mask ()$+$

scale_shape_manual(values $=c(49: 55))+$

geom_point(aes(colour $=$ factor(Year),

shape=factor(Affaldsselskab,levels $=c($ "Greater Copenhagen",

"Central Zealand","Djursland","Silkeborg") )),size=2) +

\#scale_shape_manual(values $=c(103,99,100,115))+$

theme_bw ()+

theme_rgbw ()$+$

theme_rgbw(base_size $=11$,base_family $=$ "')+ 
labs(shape="Study areas",colour="Years")+

scale_T_continuous $($ limits $=c(0.0, .8))+$

scale_L_continuous $($ limits $=c(0.0,0.8))+$

scale_R_continuous $($ limits $=c(0.2,1))$

\#END of Figure 7

\#\#\%\%\%\%\%\%\%\%\%\%\%\%\%\%\%\%\%\%\%\%\%\%\%\%\%\%\%\%\%\%\%\%\%\%\%\%\%\%\%\%\%\%\%\%\%\%\%\%\%\%\%\%\%\%\%\%

\%\%\%\%\%\%\%\%\%\%\%\%\%\%\%\%\%\%\%\%\%\%

\#\%\%\%\%\%\%\%\%\%\%\%\%\%\%\%\%\%\%\%\%\%\%\%\%\%\%\%\%\%\%\%\%\%\%\%\%\%\%\%\%\%\%\%\%\%\%\%\%\%\%\%\%\%\%\%\%\%

\%\%\%\%\%\%\%\%\%\%\%\%\%\%\%\%\%\%\%\%\%\%

unique(wrc2018_v8\$Fraction)\%>\%View()

\#Data for correlation analysis

library(readxl)

DK_all <-read_excel("H:/Paper and Article/Paper_HRC/data_all/DK_all.xlsx", sheet = "Sheet1")

table2_4=

table3_1\%>\%

bind_cols(Maengde $=$ rep $(0$, nrow $($ table3_1) $)) \%>\%$

bind_rows(table3_2)\%>\%

left_join (

DK_all\%>\%\#\#Data cleaning and combination

mutate $($ Kommune $=$

str_replace_all(Kommune,\#Find all danish characters and replace them with

\#The tricky part is to remember to have mutate at the beginning

\#For more inf. page 215, $\mathrm{R}$ for Data Science

c("æ"="ae","å"="aa","ø"="oe","Å"="Aa","Ø"="Oe","Æ"="Ae")))\%>\%

gather(Year, Values, ‘2010``2017`)\%>\%

mutate(Pers=fct_recode(Pers,

"7 personer"="7 personer og derover"))\%>\%

separate(Pers,c("Number_pe","Per"),sep = " ")\%>\%

mutate(Number_pe=as.double(Number_pe))\% $\% \%$

mutate(Housing_type=ifelse(Housing\%in\% "Etageboliger","MF","SF"))\%>\%

mutate $(\mathrm{Pp}=$ Values*Number_pe $) \%>\%$

group_by(Housing_type,Kommune,Year)\%>\%

summarise(Person=sum(Pp),Household=sum(Values) $) \%>\%$

gather(Paaa, tot,c(Person,Household) $\%>\%$

unite(Housing,Housing_type,Paaa,sep = "_")\%>\%

spread(Housing,tot))

write_xlsx(table2_4, "H:/Paper and Article/Paper_HRC/Results/2018-05-Results/table2_4.xlsx")

\#\#SM Figure

\#Number of visitor per household and number of visitors per inhabitant

table2_4\%>\%

mutate(visit_ho=Visitors/Household,visit_per=Visitors/Person)\% $\% \%$ 
select(Year,Affaldsselskab,Kommune,visit_ho,visit_per)\%>\%

gather(visti,ratio,c(visit_ho,visit_per))\%>\%

ungroup()\%>\%

rename("Municipalities"="Kommune")\%>\%

mutate(visti=fct_recode(visti,

"Visitor per household"="visit_ho",

"Visitor per inhabitant"="visit_per"))\%>\%

ggplot(aes(Year,ratio))+

geom_line(aes(group=Municipalities, colour=Municipalities),size=1.2)+

scale_shape_manual(values=97:109)+

geom_point(aes(group=Municipalities, shape=Municipalities),size=3)+

facet_wrap $(\sim$ factor(visti), scales $=$ "free")+

theme_bw ()$^{+}$

\#labs(colour="Municipalities",shap)+

theme(text = element_text $($ colour = "black", face = "bold", size = 12) $)+$

theme $($ axis.text $=$ element_text $($ color $=$ "black", size $=12))$

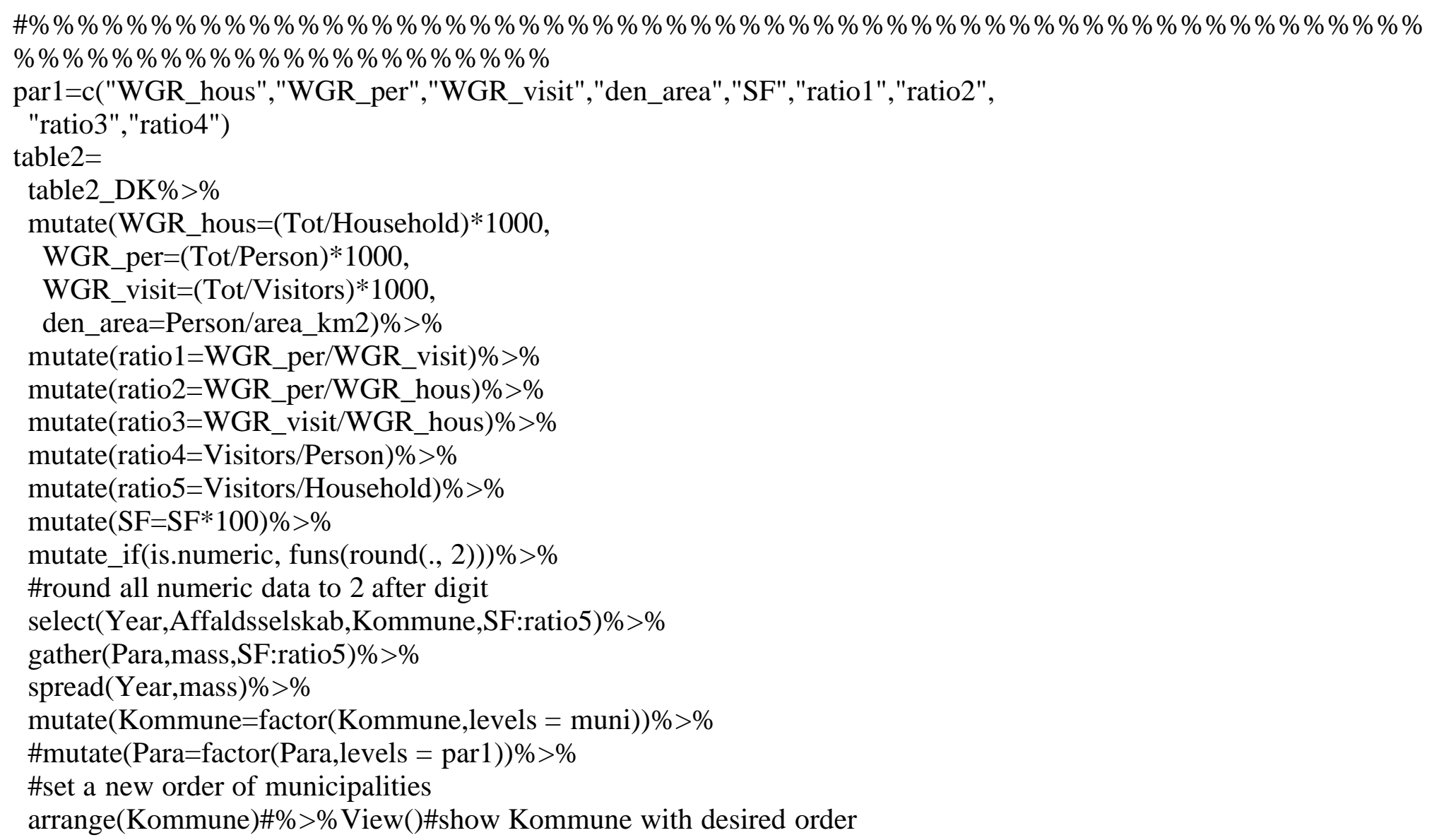


library(psych)

\#\#Summary: descriptive statisics

table2\% $\% \%$

gather(Year,Mass, `2010``2016`)\%>\%

group_by(Para)\%>\%

do(describe(.\$Mass) $) \%>\%$

mutate(CV=sd/mean*100)

wrc2018_v8\%>\%

group_by(Year,Affaldsselskab,Genbrugspladser,Treatment)\%>\%

summarise(tot=sum(Maengde) $\%>\%$

group_by(Year,Affaldsselskab,Genbrugspladser)\%>\%

transmute(Treatment, Co1=tot/sum(tot)*100)\%>\%\#head()

group_by(Year,Treatment)\%>\%

do(describe(.\$Co1))\%>\%View()

\#++++++Graph showing descriptive statistics

table2\%>\%

filter(Para\%in\% c("WGR_per","WGR_visit","WGR_hous"))\%>\%

gather(Year,Mass, '2010`:2016”)\%>\%

mutate(Para=fct_recode(Para,

"WGR Person"="WGR_per","WGR Houseold"="WGR_hous",

"WGR Visitor"="WGR_visit"))\%>\%

ggplot(aes( $\mathrm{x}=$ factor(Year), $\mathrm{y}=$ Mass))+

scale_shape_manual(values=1:nlevels(table2\$Kommune))+

geom_boxplot()+

geom_point(aes(shape=Kommune $))+$

facet_wrap $(\sim$ Para,scales $=$ "free" $)+$

theme_bw( $)+$

labs(colour="Municipalities",x="Year",y="Generation rates")+

\#labs(x="Number of inhabitants per km2", $y=$ "ratio")+

\#theme(legend.position="top")+

theme(text =element_text $($ colour = "black", face = "bold", size = 12)

\#++++++Graph showing descriptive statistics

table2\% $>\%$

filter(Para\%in\% c("WGR_per","WGR_visit","WGR_hous"))\%>\%

gather(Year,Mass, '2010`:2016')\%>\%

mutate(Para=fct_recode(Para,

"WGR Person"="WGR_per","WGR Houseold"="WGR_hous",

"WGR Visitor"="WGR_visit"))\%>\%

ggplot(aes( $\mathrm{x}=$ factor(Year), $\mathrm{y}=$ Mass))+

\#scale_shape_manual(values=1:nlevels(table2\$Kommune))+

geom_boxplot()+

geom_point(aes(color=Kommune) $)+$

facet_wrap $(\sim$ Para,scales $=$ "free")+

theme_bw( $)^{+}$

labs(colour="Municipalities",x="Year",y="Generation rates")+

\#labs(x="Number of inhabitants per km2", $y=$ "ratio")+

\#theme(legend.position="top")+

theme $($ text =element_text $($ colour = "black", face = "bold", size = 12) $)$ 
\#Graph to show

table2_DK\% $>\%$

mutate(WGR_hous=(Tot/Household)*1000,

WGR_per=(Tot/Person)*1000,

WGR_visit $=($ Tot/Visitors $) * 1000$,

den_area=Person/area_km2)\% $\% \%$

mutate(ratio1=WGR_per/WGR_visit)\%>\%

mutate(ratio2=ratio1/den_area)\% $>\%$

mutate_if(is.numeric, funs(round(., 2)))\% $\%$ \%

ggplot(aes(x=den_area,y=ratio1))+geom_point()+

geom_text(aes(label $=$ Kommune),hjust $=0$, vjust $=0)+$

geom_smooth(method="loess")+theme_bw()+

theme_bw()+

\#labs(x="Number of inhabitants per km2", $y=$ "ratio")+

\#theme(legend.position="top")+

theme(text =element_text $($ colour = "black", face = "bold", size = 12) $)$

\#+++++++++++++++++++++++++++++++++++++++++++

table2_DK\%>\%

mutate(WGR_hous=(Tot/Household)*1000,

WGR_per $=($ Tot/Person $) * 1000$,

WGR_visit $=($ Tot/Visitors $) * 1000$,

den_area=Person/area_km2)\% $>\%$

mutate(ratio1=WGR_per/WGR_visit)\%>\%

mutate(ratio2=ratio1/den_area)\% $>\%$

mutate_if(is.numeric, funs(round(., 2)) $\%>\%$

$\operatorname{ggplot}($ aes $(\mathrm{x}=$ Visitors, $\mathrm{y}=(\mathrm{SF} *$ Household $)))+$ geom_point ()$^{+}$

geom_text(aes(label=Kommune),hjust $=0$, vjust $=0)+$

geom_smooth(method="lm")+theme_bw()+

theme_bw( $)+$

\#labs(x="Number of inhabitants per km2", $y=$ "ratio")+

\#theme(legend.position="top")+

theme(text =element_text $($ colour = "black", face $=$ "bold", size $=12)$ )

$\mathrm{Z}+++++++++++++++++++++++++++++++++++++++++++++++++++++++++++++++++++++++++++++++$

$\mathrm{\# +++++++++++++++++++++++++++++++++++++++++++++++++++++++++++++++++++++++++++++++}$

table2_DK\% $>\%$

mutate(WGR_hous=(Tot/Household)*1000,

WGR_per=(Tot/Person)*1000,

WGR_visit $=($ Tot/Visitors $) * 1000$,

den_area=Person/area_km2)\%>\%

mutate(ratio1=WGR_per/WGR_visit)\% $>\%$

mutate(ratio2=ratio1/den_area) $\%>\%$

mutate_if(is.numeric, funs(round(., 2)))\%>\%

$\operatorname{ggplot}(\operatorname{aes}(\mathrm{x}=$ ratio1,y=(SF), colour=factor(Year $)))+$

geom_point ()$^{+}$

geom_text(aes(label=Kommune),hjust $=0$, vjust $=0)+$

geom_smooth(method="lm")+theme_bw()+

theme_bw( $)+$

\#labs(x="Number of inhabitants per km2", $y=$ "ratio")+

\#theme(legend.position="top")+

theme $($ text =element_text $($ colour = "black", face = "bold", size = 12) $)$ 
mutate(WGR_hous=(Tot/Household)*1000,

WGR_per $=($ Tot/Person $) * 1000$,

WGR_visit $=($ Tot/Visitors $) * 1000$,

den_area=Person/area_km2)\% $\% \%$

mutate(ratio1=WGR_per/WGR_visit)\%>\%

mutate(ratio2=ratio1/den_area) $\%>\%$

mutate_if(is.numeric, funs(round(., 2)))\%>\%

$\operatorname{ggplot}(\operatorname{aes}(\mathrm{x}=$ ratio1, $\mathrm{y}=(\mathrm{SF})))+$

geom_point ()$^{+}$

geom_text(aes(label=Kommune),hjust $=0$, vjust $=0)+$

geom_smooth(method="lm")+theme_bw()+

theme_bw ()$^{+}$

\#labs(x="Number of inhabitants per km2", $y=$ "ratio")+

\#theme(legend.position="top")+

theme(text =element_text(colour = "black", face = "bold", size =12))

\#+++++++++++++++++++++++++++++++++++++++++++++++++++++++++++++++++++++++++++++++

table2_DK\% $>\%$

mutate(WGR_hous=(Tot/Household)*1000,

WGR_per=(Tot/Person)*1000,

WGR_visit=(Tot/Visitors)*1000,

den_area=Person/area_km2)\% $\% \%$

mutate(ratio1=WGR_per/WGR_visit)\%>\%

mutate(ratio2=ratio1/den_area)\% $>\%$

mutate_if(is.numeric, funs(round(., 2)))\%>\%

$\operatorname{ggplot}(\operatorname{aes}(\mathrm{x}=($ den_area $), \mathrm{y}=$ ratio1 $))+$

geom_point ()$^{+}$

geom_text(aes(label=Kommune),hjust $=0$, vjust $=0)+$

geom_smooth(method="lm")+theme_bw()+

theme_bw ()$^{+}$

\#labs(x="Number of inhabitants per km2", $y=$ "ratio")+

\#theme(legend.position="top")+

theme(text =element_text $($ colour = "black", face = "bold", size = 12) $)$

\#+++++++++++++++++++++++++++++++++++++++++++++++++++++++++++++++++++++++++++++++

table2_DK\% $>\%$

mutate(WGR_hous=(Tot/Household)*1000,

WGR_per=(Tot/Person)*1000,

WGR_visit=(Tot/Visitors)*1000,

den_area $=$ Person/area_km2)\% $\% \%$

mutate(ratio1=WGR_per/WGR_visit)\%>\%

mutate(ratio2=ratio1/den_area)\% $>\%$

mutate_if(is.numeric, funs(round(., 2)))\%>\%

ggplot(aes(x=Visitors,y=SF))+geom_point()+

geom_text(aes(label=Kommune),hjust $=0$, vjust $=0)+$

\#geom_smooth(method="lm")+theme_bw()+

theme_bw( $)^{+}$

\#labs(x="Number of inhabitants per km2", y="ratio")+

\#theme(legend.position="top")+

theme $($ text =element_text $($ colour = "black", face = "bold", size = 12) 
\#Build a 3 D plot

library(scatterplot3d)

scatterplot3d(table2_4\$Visitors, table2_4\$SF_house, log(table2_4\$den_area))

pairs(data.frame(table2_4\$Visitors, table2_4\$SF_house, log(table2_4\$den_area)),

panel=panel.smooth, oma $=\operatorname{rep}(2,4))$

\#3Testing factors affecting the number of visitors

$\#++++++$ This is the best

fm1 $=\operatorname{lm}\left((\right.$ Visitors $) \sim\left(S F \_h o u s e\right)+\log ($ den_area $)$, data =table2_4 )

$\mathrm{fm} 2=\operatorname{lm}(($ Visitors $) \sim($ SF_house $)$, data $=$ table2_4 $)$

fm3 $=\operatorname{lm}\left((\right.$ Visitors $) \sim\left(S F \_h o u s e\right)+\left(M F \_h o u s e\right)+\log ($ den_area $)$, data =table2_4 )

anova(fm3,fm1)

$\operatorname{cbind}(\operatorname{coef}(\mathrm{fm}))$

summary $\left(\operatorname{lm}\left((\right.\right.$ Visitors $) \sim\left(S F \_h o u s e\right)+\left(M F \_h o u s e\right)+\log ($ den_area $)$, data =table2_4 $\left.)\right)$

summary $\left(\operatorname{lm}\left((\right.\right.$ Visitors $) \sim\left(S F \_h o u s e\right)+\log ($ den_area $)$, data =table2_4 ))

anova(lm((Visitors) I(SF*Person)+log(den_area), data =table2_4 ))

drop1(lm((Visitors) I(SF*Person)+log(den_area), data =table2_4 ))

confint(lm((Visitors) I(SF*Person)+log(den_area), data =table2_4 ))

\#\%\%\%\%\%\%\%\%\%\%\%\%\%\%\%\%\%\%\%\%\%\%\%\%\%\%\%\%\%\%\%\%\%\%\%\%\%\%\%\%\%\%\%\%\%\%\%\%\%\%\%\%\%\%\%\%\%

\%\%\%\%\%\%\%\%\%\%\%\%\%\%\%\%\%\%\%\%\%\%

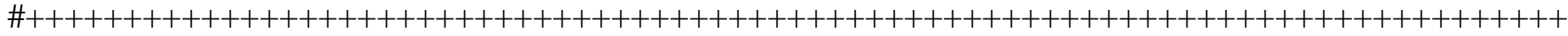

$\mathrm{Z}+++++++++++++++++++++++++++++++++++++++++++++++++++++++++++++++++++++++++++++++$

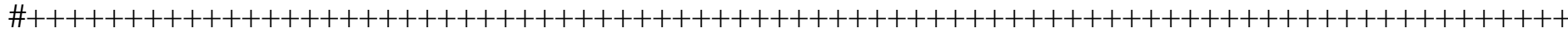

\#Decomposition of waste generation for the four areas

library(ggseas)

library(lubridate)

yearcol=c("\#d11141","\#00b159", "\#00aedb","\#f37735","\#ffc425","\#cccccc","\#8c8c8c")

$\mathrm{Z}+++++++++++++++++++++++++++++++++++++++++++++++++++++++++++++++++++++++++++++++$

\#\#All waste

wrc2018_v8\$Affaldsselskab=factor(wrc2018_v8\$Affaldsselskab,,

levels = c("Greater Copenhagen","Central Zealand","Djursland","Silkeborg"))

wrc2018_v8\%>\%

mutate(date1=ymd( paste(Year, Months, "15", sep="-")))\%>\%

group_by(date1,Affaldsselskab)\%>\%\#head()

summarise(tot=sum(Maengde) 1000 )\%>\%head()

mutate(Affaldsselskab=fct_recode(Affaldsselskab,

"Central Zealand"="Roskilde"))\%>\%head()

ggsdc(aes( $\mathrm{x}=$ date1,y=tot, colour=Affaldsselskab),

method $=$ "stl", s.window $=7$, frequency $=12$,

facet.titles = c("The original series", "The underlying trend",

"Regular seasonal patterns", "All the randomness left")) +

theme_bw()+

geom_line $($ size $=1.2)+$

labs(colour="Study areas",y="1000 Mg",x="Study period")+

theme(legend.position="top")+

theme(text $=$ element_text $($ colour $=$ "black", face $=$ "bold", size = 12) $)+$

theme $($ axis.text $=$ element_text $($ color $=$ "black", size $=12)$ )

$\mathrm{Z}+++++++++++++++++++++++++++++++++++++++++++++++++++++++++++++++++++++++++++++++$

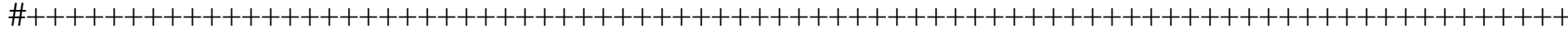
$\mathrm{Z}+++++++++++++++++++++++++++++++++++++++++++++++++++++++++++++++++++++++++++++++$ \#Combine waste data and number of visitors 
Waste_visit_v1=

wrc2018_v8\%>\%

group_by(Year,Months,date1,Affaldsselskab,Kommune,Genbrugspladser)\%>\%

summarise(tot $=$ sum(Maengde) $) \%>\%$

left_join(

visit_v1\%>\%mutate(Year=as.double(Year))

)\%> \%select(-Type)

\#\#Compute ratio and boxplot

Waste_visit_v1\%>\%

\#Compute aeverage per month

group_by(Months,Genbrugspladser)\%>\%

summarise(tot1=mean(tot), visit1=mean(Visit)) $\%>\%$

\#Compute average per HWRC

group_by(Genbrugspladser)\%>\%

summarise(tot2=mean(tot1), visit2=mean(visit1) $\%>\%$

\#Join

right_join(

Waste_visit_v1\%>\%

group_by(Year,Months,Genbrugspladser)\% $\% \%$

summarise(mass $=$ sum(tot),beso $=$ sum(Visit) $\%>\%$

group_by(Months,Genbrugspladser)\%>\%

summarise(tot1=mean(mass), visit1=mean(beso)) $) \%>\%$

mutate(ratio1=tot1/tot2, ratio_be=visit1/visit2)\% $>\%$

mutate $($ Waste $=$ ifelse $($ ratio1 $>1,(($ ratio1-1)*100), $(-1 *(1-$ ratio1)*100) $)) \%>\% \#$ head()

\#We compute mean ratio ratio2 for mass and ratio_be2 for visitor

mutate(Visitors= ifelse(ratio_be $>1$, ((ratio_be-1)*100),(-1*(1-ratio_be)*100)) $) \%>\%$

\#\#Now we select only the mean ratios ratio2 for mass and ratio_be2 for visitor

select(Genbrugspladser,Months,Waste,Visitors)\%>\%

\#We combine both ratios

gather(Ratio,tal, Waste:Visitors) $\%>\%$

ggplot(aes( $\mathrm{x}=$ Months, $\mathrm{y}=$ tal $))+$

geom_boxplot(aes(colour=Ratio),position=position_dodge(.9))+

stat_summary(fun. $y=$ mean, colour="darkred", geom="point",

aes(group=Ratio), position=position_dodge(.8),

shape $=18$, size $=3$, show_guide $=$ FALSE $)+$

geom_hline $($ yintercept $=0$, colour="red",size $=1)+$

theme_bw ()$^{+}$

theme(legend.position="top")+

labs(x="Months", $y=$ "Mean ratio in \%")+

theme $($ text $=$ element_text $($ colour $=$ "black", face $=$ "bold", size = 12) $)$

\#\%\%\%\%\%\%\%\%\%\%\%\%\%\%\%\%\%\%\%\%\%\%\%\%\%\%\%\%\%\%\%\%\%\%\%\%\%\%\%\%\%\%\%\%\%\%\%\%\%\%\%\%\%\%\%\%\% \%\%\%\%\%\%\%\%\%\%\%\%\%\%\%\%\%\%\%\%\%\%\%

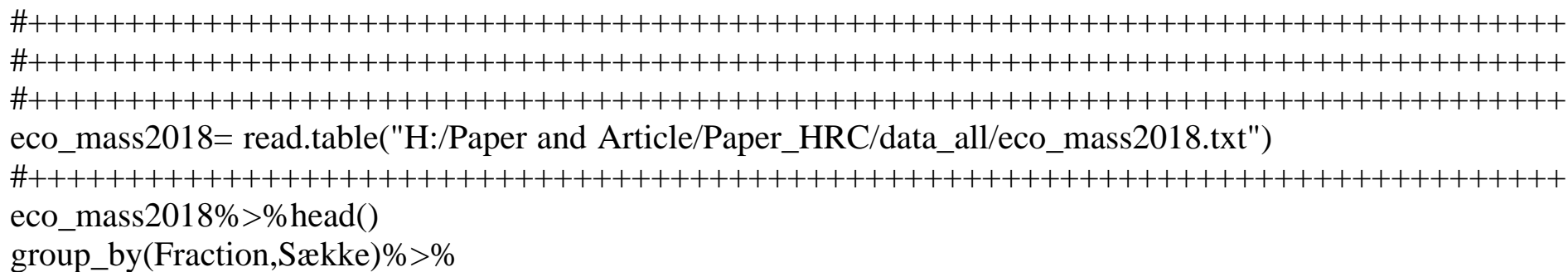




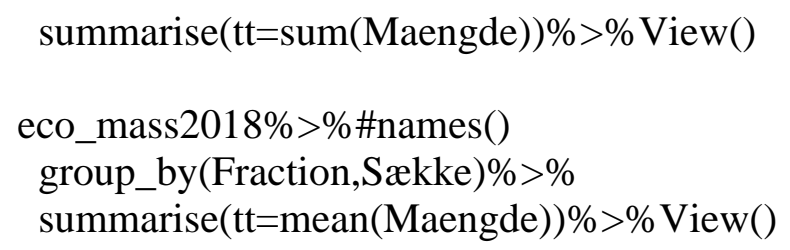


"Grenaa"="Lemvig",

"Vermlandsgade"="Hvidovre_II"))\%>\%

mutate(Treatment=ifelse(Fraction\%in\%c("Residual Household waste",

"Miscellaneous combustible"),"Incineration",

ifelse(Fraction\%in\% c("Untreated wood",

"Landfilled waste","Small household appliances",

"Chemichal fertilizer","Treated wood",

"Polyvinylchloride (PVC)"),"Other","Recycling")))\%>\%

group_by(Genbrugspladser,Treatment,type)\%>\%

summarise(tot1=sum(Maengde) $\%>\%$

group_by(Genbrugspladser,type)\%>\%

transmute(Treatment,tot1_per=tot1/sum(tot1))\#\%>\%head()

)$\%>\% \#$ head()

mutate(Pot0=tot1_per*Mass)

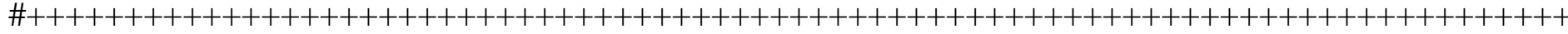

\#

potent_dat $=$

Inc_port\%>\%select(-c(Mass,tot1_per))\%>\%

rename(Mass $=$ Pot 0$) \%>\%$

select(Year,Genbrugspladser, Treatment,type,Mass)\%>\%

mutate(Treatment=as.factor(Treatment))\%>\%

bind_rows(

wrc_eco $\%>\%$

add_column(type=rep("old",nrow(wrc_eco)) $) \%>\%$

mutate(Pot0='2016`)\%> $>$ select(-c(per_Tom,Pot) $\%>\%$ \#head()

gather(Year,Mass,-c(Fraktioner:Genbrugspladser,type))\%>\%

group_by(Year,Genbrugspladser,Treatment,type)\%>\%

summarise(Mass=sum(Mass))\% $\% \%$

mutate(Me2=ifelse(Year \%in\% "Pot0"\&Treatment \%in\% "Incineration","0","1"))\%>\%

filter(Me2\%in\%"1")\%>\%select(-Me2)

)$\%>\#$

group_by(Year,Genbrugspladser,Treatment)\%>\%

summarise(MassMg=sum(Mass))\%>\%spread(Year,MassMg)

\#Suite of calcuati

library(ggtern)

Yearcol=c("\#140A00","\#2121D9", "\#9999FF", "\#D92121", "\#21D921", "\#FFFF4D", "\#FF9326","\#963700")

tt1=c("black","navy","orangered","purple4","blue","deeppink4","green4","red")

potent_dat $\%>\%$ as.data.frame()\% $\% \%$

gather(Year,Mass, -c(Genbrugspladser,Treatment))\%>\%\#head()

spread(Treatment,Mass)\%>\%\#head()

ggtern(aes( $\mathrm{y}=$ Incineration, $\mathrm{x}=$ Other, $\mathrm{z}=$ Recycling $))+$

geom_mask ()$+$

scale_shape_manual(values $=1: 7)+$

geom_point(aes(colour $=$ factor $($ Year $)$, shape $=$ Genbrugspladser $),$ size $=2)+$

\#geom_line $($ aes $($ colour $=$ factor $($ Genbrugspladser $))+$

theme_bw ()$+$ theme_rgbw ()$+$

theme_rgbw(base_size $=11$, base_family $=$ "')+

scale_colour_manual $($ values $=\mathrm{tt} 1)+$

\#scale_colour_brewer(palette="set2")+

\#scale_colour_gradient(colours=rainbow(8))+

labs(shape $=$ "Areas",colour="Years")+

scale_T_continuous $($ limits $=\mathrm{c}(0.0, .5))+$

scale_L_continuous $($ limits $=c(0.0,0.5))+$ 
scale_R_continuous $($ limits $=c(0.5,1))$

\#\#Reduce to three years

potent_dat $\%>\%$ as.data.frame()\% $\% \%$

gather(Year,Mass,-c(Genbrugspladser, Treatment))\%>\%\#head()

spread(Treatment,Mass)\%>\%filter(Year\%in\%c("2015","2016","Pot0"))\%>\%

ggtern(aes( $\mathrm{y}=$ Incineration, $\mathrm{x}=$ Other, $\mathrm{z}=$ Recycling $)+$

geom_mask ()$+$

scale_shape_manual(values $=c(49: 55))+$

geom_point(aes(colour $=$ factor(Year),shape=Genbrugspladser),size=4) +

\#geom_line(aes(colour $=$ factor(Genbrugspladser $)))^{+}$

theme_bw ()$+$ theme_rgbw ()$+$

theme_rgbw(base_size $=11$,base_family $=$ "'")+

scale_colour_manual(values = c("blue","green4","red"))+

\#scale_colour_brewer(palette="set2")+

\#scale_colour_gradient(colours=rainbow(8))+

labs(shape="Areas",colour="Years")+

scale_T_continuous $($ limits $=c(0.0, .5))+$

scale_L_continuous $($ limits $=c(0.0,0.5))+$

scale_R_continuous $($ limits $=c(0.5,1))$

\#Total Waste per area

Area_year=wrc2018_v8 \%>\%

group_by(Year,Affaldsselskab)\% $>\%$

summarise(waste_year=sum(Maengde)/1000)\%>\%

spread(Affaldsselskab,waste_year,fill = 0)

setwd("H:/Paper and Article/Paper_HRC/Results")

library(writexl)

write_xlsx(Area_year, "Area_year.xlsx")

\#\#Exclude gardening waste

wrc2018_v8\%>\%\#distinct(Fraction)\%>\%arrange(Fraction)

filter(Fraction\%!in\% "Garden waste")\%>\%

mutate(date1=ymd( paste(Year, Months, "15", sep="-")))\%>\%

group_by(date1,Affaldsselskab)\%>\%\#head()

summarise(tot=sum(Maengde)/1000)\%>\%\#head()

ggsdc(aes( $\mathrm{x}=$ date $1, \mathrm{y}=$ tot, colour $=$ Affaldsselskab),

method $=$ "stl", s. window $=7$, frequency $=12$,

facet.titles = c("The original series", "The underlying trend",

"Regular seasonal patterns", "All the randomness left")) +

theme_bw ()+

geom_line $($ size $=1.2)+$

labs(colour="Study areas",y="1000 Mg (Without garden waste)",x="Study period")+

theme(legend.position="top")+

theme $($ text $=$ element_text $($ colour $=$ "black", face $=$ "bold", size = 12) $)+$

theme $($ axis.text $=$ element_text $($ color $=$ "black", size $=12)$ )

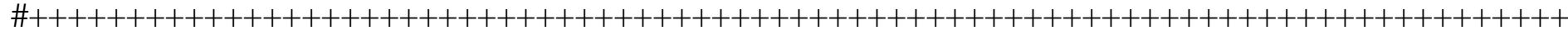

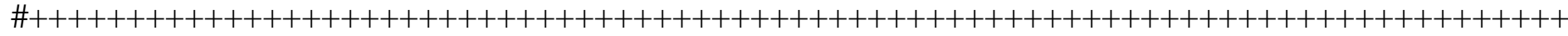
\#+++++++++++++++++++++++++++++++++++++++++++++++++++++++++++++++++++++++++++++++ \# Ratio analysis: it is based on arithmetic mean ratio \#Cowpertwait and Metcalfe, (2009), page9 


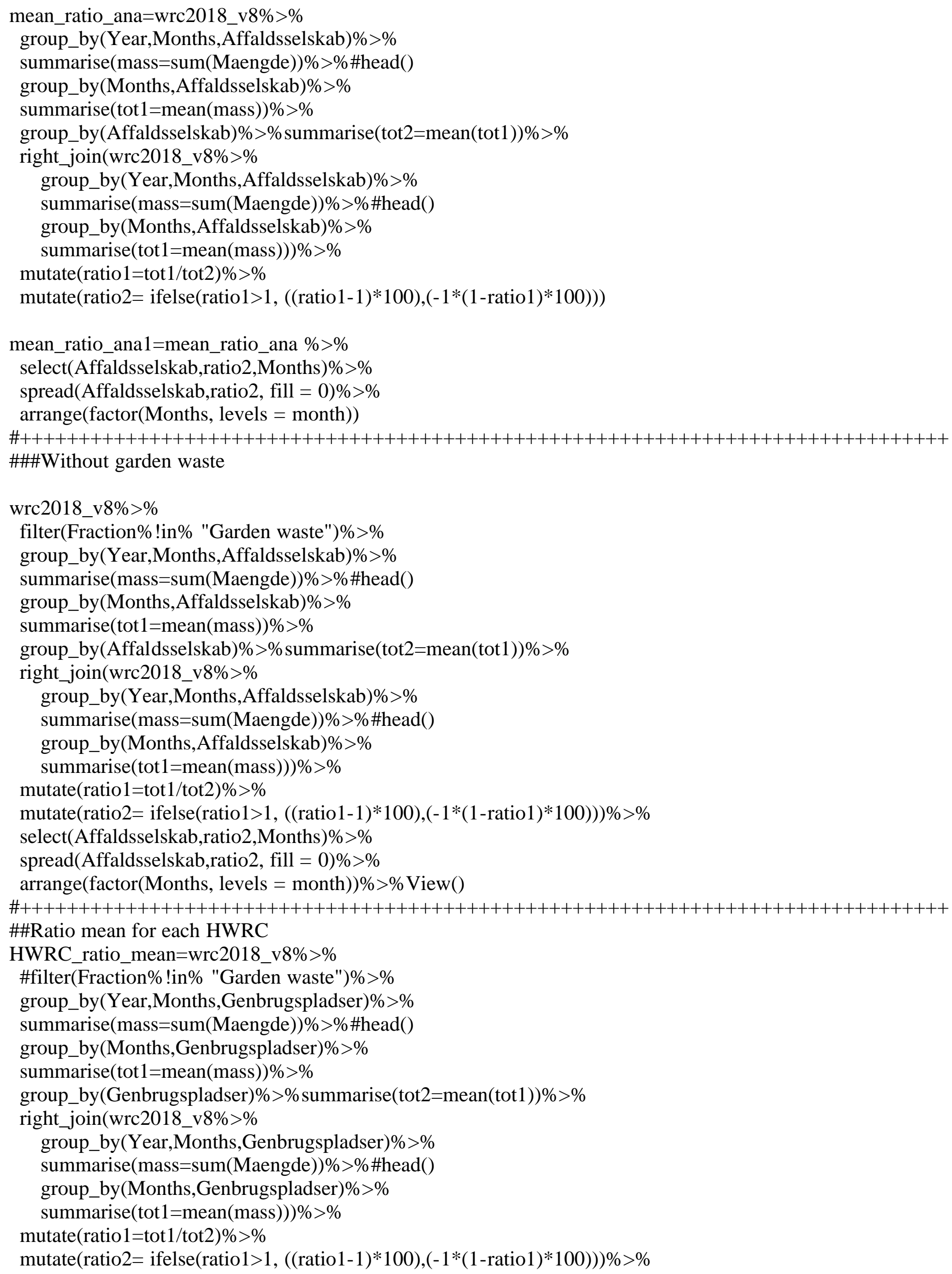


select(Genbrugspladser,ratio2,Months) $\%>\%$

spread(Genbrugspladser,ratio2, fill $=0$ ) $\%>\%$

arrange (factor(Months, levels = month) $\# \%>\% \operatorname{View}()$

setwd("H:/Paper and Article/Paper_HRC/Results")

library(writexl)

write_xlsx(HWRC_ratio_mean, "HWRC_ratio_mean.xlsx")

\#+++++++++++++++++++++++++++++++++++++++++++++++++++++++++++++++++++++++++++++++

\#Boxplot for ratio mean for all HWRC

wrc2018_v8\%>\%

\#filter(Fraction\%!in\% "Garden waste")\%>\%

group_by(Year,Months,Genbrugspladser)\%>\%

summarise(mass=sum(Maengde))\% $\% \% \#$ head()

group_by(Months,Genbrugspladser)\%>\%

summarise(tot1=mean(mass) $\%>\%$

group_by(Genbrugspladser)\%>\%summarise(tot2=mean(tot1))\%>\%

right_join(wrc2018_v8\%>\%

group_by(Year,Months,Genbrugspladser)\%>\%

summarise(mass $=$ sum(Maengde) $) \%>\% \#$ head()

group_by(Months, Genbrugspladser)\%>\%

summarise(tot1=mean(mass) $)) \%>\%$

mutate(ratio1=tot $1 /$ tot 2$) \%>\%$

mutate $($ ratio2 $=$ ifelse $($ ratio1 $>1,(($ ratio1-1)*100), $(-1 *(1-$ ratio1 $) * 100))) \%>\% \#$ head()

ggplot(aes( $\mathrm{x}=$ Months, $\mathrm{y}=$ ratio2 $))+$

geom_boxplot( $)+$

stat_summary(fun.y=mean, colour="darkred", geom="point",

shape $=18$, size $=3$, show_guide $=$ FALSE $)+$

geom_hline(yintercept $=0$, colour="red")+

labs(x="Months",y="Mean ratio")+

theme_classic()+

labs(x="Months", $\mathrm{y}=$ "Mean ratio in \%")+

theme(text =element_text $($ colour = "black", face = "bold", size = 12)

\#+++++++++++++++++++++++++++++++++++++++++++++++++++++++++++++++++++++++++++++++

\#Boxplot for ratio mean for all HWRC subdivided in area

wrc2018_v8\%>\%

\#filter(Fraction\%!in\% "Garden waste")\%>\%

group_by(Year,Months,Affaldsselskab,Genbrugspladser)\%>\%

summarise(mass=sum(Maengde) $\%>\% \#$ head()

group_by(Months,Affaldsselskab,Genbrugspladser)\%>\%

summarise(tot1=mean(mass) $\%>\%$

group_by(Affaldsselskab,Genbrugspladser)\%>\%summarise(tot2=mean(tot1))\%>\%

right_join(wrc2018_v8\%>\%

group_by(Year,Months,Affaldsselskab,Genbrugspladser)\%>\%

summarise(mass=sum(Maengde) $\%$ \% $\%$ \#head()

group_by(Months,Affaldsselskab,Genbrugspladser)\%>\%

summarise(tot1=mean(mass) $)$ )\% $>\%$

mutate(ratio1=tot $1 /$ tot 2$) \%>\%$

mutate $($ ratio2 $=$ ifelse $($ ratio1 $>1,(($ ratio1-1)*100), $(-1 *(1-$ ratio1)*100) $)) \%>\% \#$ head()

\#filter(ratio2<100)\% $>\%$

ggplot(aes( $\mathrm{x}=$ Months, $\mathrm{y}=$ ratio2 $))+$

geom_boxplot(aes(fill=factor(Affaldsselskab)),size=.5)+

stat_summary(fun.y=mean, colour="darkred", geom="point",

aes(group=Affaldsselskab), position=position_dodge(.7),

shape $=18$, size $=3$, show_guide $=$ FALSE +

geom_hline(yintercept $=0$, colour="red",size=1)+ 


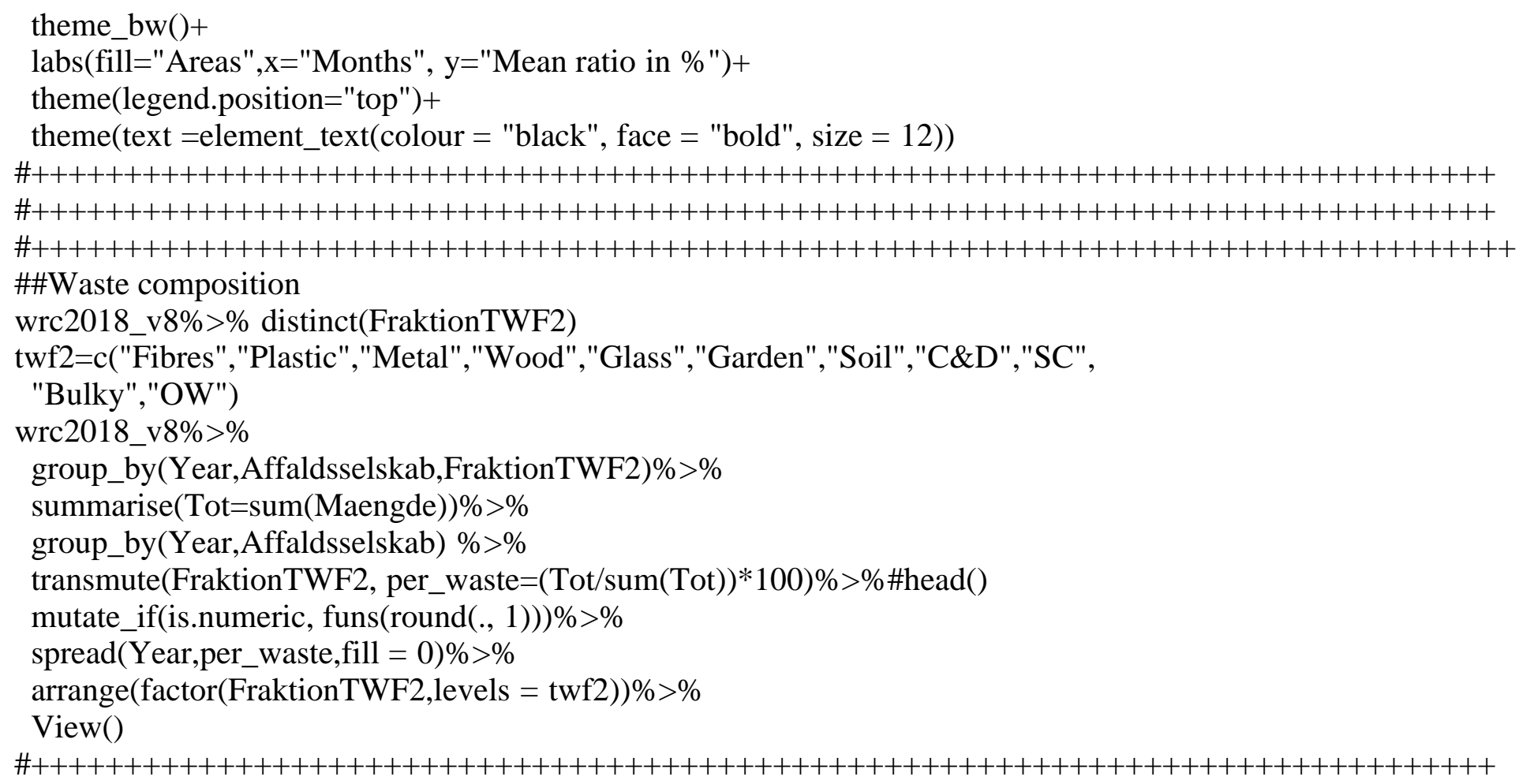




\begin{abstract}
summarise(Tot=sum(Maengde) $\%>\%$
\end{abstract}
group_by(Year,Affaldsselskab,Kommune,Genbrugspladser) \%>\%

transmute(Fraktioner2, per_waste $=($ Tot $/$ sum(Tot) $) * 100) \%>\%$

mutate_if(is.numeric, funs(round(., 1)))\%>\%

spread(Fraktioner2,per_waste,fill $=0$ )

setwd("H:/Paper and Article/Paper_HRC/Results")

library(writexl)

write_xlsx(Biplot_Fraktioner2, "Biplot_Fraktioner2.xlsx")

Biplot_Fraktioner2\%>\%head()

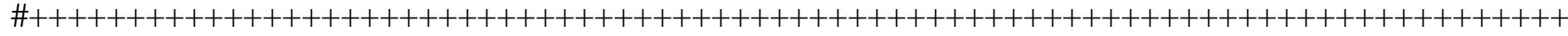

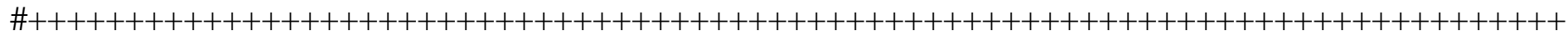
\#+++++++++++++++++++++++++++++++++++++++++++++++++++++++++++++++++++++++++++++++ selk=c("Copenhagen","Roskilde","Djursland","Silkeborg")

bar_treat $=$ wrc2018_v8\%>\%

group_by(Year,Affaldsselskab,Treatment)\%>\%\#head() summarise(Tot=sum(Maengde)/1000)\%>\%\#head()

group_by(Year,Affaldsselskab) \%>\%

transmute(Treatment, per_waste=(Tot/sum(Tot)*100))\%>\%\#head()

ggplot(aes(x=factor(Year),y=(per_waste),

fill=factor(Treatment,levels = c("Other","Incineration","Recycling"))))+

geom_bar(position="fill",stat="identity")+ \#geom_label(stat = "fill_labels")+

facet_wrap $(\sim$ factor(Affaldsselskab,levels $=$ selk $)$, scales $=$ "free")+

theme_bw ()$^{+}$

theme(legend.text=element_text(size=12))+

theme(legend.position="top")+

scale_y_continuous(labels = function(x) paste0(x*100, "\%"))+

scale_fill_manual("Waste treatments", values = c("blue","red","green4"))+

guides(title="Waste treatments", fill = guide_legend $($ nrow $=1))+$

theme(text $=$ element_text $($ colour = "black", face = "bold", size = 12) $)+$

theme $($ axis.text $=$ element_text $($ color $=$ "black", size =12) $)+$

theme(legend.text=element_text(size=12))+

theme(strip.text = element_text $($ color $=$ "black", size $=12)$,

strip.background = element_rect(color="black",fill="white"))+

ylab("Composition")+ xlab("Years")

ggsave("bar_treat.wmf",width $=30$, height $=20$, units = "cm")

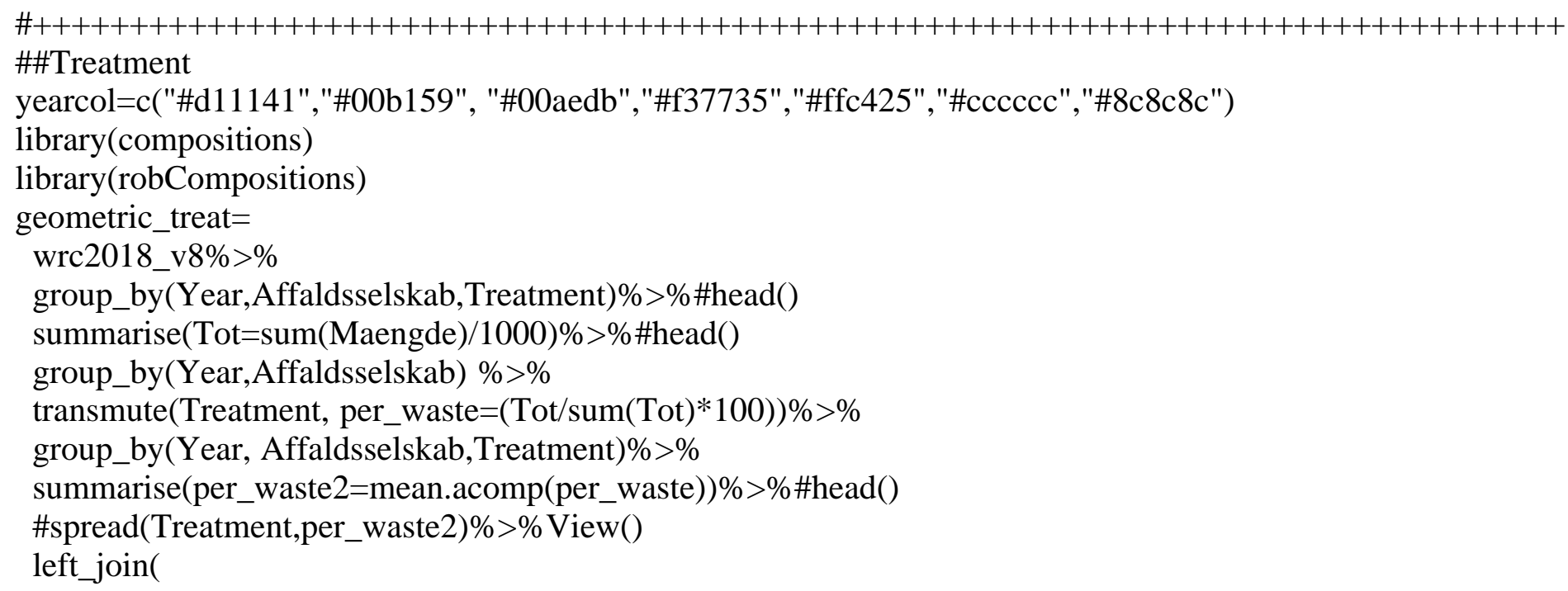


wrc2018_v8\%>\%

group_by(Year,Affaldsselskab,Treatment)\%>\%\#head()

summarise(Tot=sum(Maengde) $/ 1000) \%>\%$

group_by(Year,Affaldsselskab) \%>\%

transmute(Treatment, per_waste $=($ Tot $/$ sum(Tot) $* 100)) \%>\%$

group_by(Affaldsselskab,Treatment)\% $\% \%$

summarise(per_waste3=mean.acomp(per_waste)))\% $\% \%$

mutate(ratio=log(per_waste2/per_waste3) $\%>\%$

mutate(tto=exp(ratio) $) \%>\% \#$ head()

mutate(procent=ifelse (tto $>1$,round((tto-1)*100,0),

-round((1-tto)*100,0)))\%>\%\#head()

ggplot(aes(x=factor(Treatment,levels = c("Recycling", "Incineration","Other")),

$\mathrm{y}=$ ratio,fill=factor(Year) $))+$

geom_bar(position="dodge", stat="identity")+

geom_text(aes(x=factor(Treatment,levels = c("Recycling","Incineration","Other")),

$\mathrm{y}=$ ratio, $\mathrm{ymax}=$ ratio,

label $=$ procent,procent $=$ procent +.05 ,

\#hjust=ifelse( $\operatorname{sign}($ procent $)>0,1,0)$

vjust $=0$ ),

colour="black",position =position_dodge $($ width=0.85) $)+$

labs(colour="År")+ylab("Ratio")+xlab("Waste fraction")+

facet_wrap $(\sim$ factor(Affaldsselskab,levels = selk $)$ +\#scales = "free"

theme_bw ()$^{+}$

theme(legend.text=element_text(size=12))+

theme(legend.position="top")+

scale_fill_manual("Years", values = yearcol)+

guides(title="Years", fill = guide_legend $($ nrow $=1))+$

ylab("Ratio")+xlab("Affaldsfraktioner")+

theme $($ text $=$ element_text $($ colour $=$ "black", face $=$ "bold", size $=14))+$

theme $($ axis.text $=$ element_text $($ color $=$ "black", size $=12))+$

theme(legend.text=element_text(size=12))+

ylab("Log-ratio")+

xlab("Waste fractions")

ggsave("geometric_treat.wmf",width $=20$, height $=20$, units $=$ "cm")

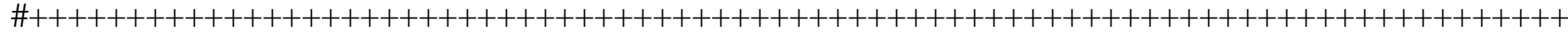

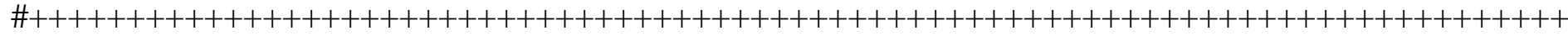
\#+++++++++++++++++++++++++++++++++++++++++++++++++++++++++++++++++++++++++++++++ library(ggtern)

wrc2018_v8\%>\%

group_by(Year,Affaldsselskab,Genbrugspladser,Treatment)\%>\%\#head()

summarise(Tot=sum(Maengde)/1000)\%>\%\#head()

group_by(Year,Affaldsselskab,Genbrugspladser) \%>\%

transmute(Treatment, per_waste $=($ Tot $/$ sum $($ Tot $) * 100)) \%>\%$

spread(Treatment, per_waste, fill $=0) \%>\%$

ungroup ()$\%>\%$

mutate(Affaldsselskab=fct_recode(Affaldsselskab,

"Central Zealand"="Roskilde"))\%>\%

\#filter(Affaldsselskab \%in\% c("Greater Copenhagen"))\%>\%

ggtern(aes( $\mathrm{y}=$ Incineration, $\mathrm{x}=$ Other, $\mathrm{z}=$ Recycling $)$ )+

geom_mask ()$+$

scale_shape_manual(values $=c(49: 55))+$

geom_point(aes(colour = factor(Year),

shape $=$ factor(Affaldsselskab,levels $=\mathrm{c}($ "Copenhagen", 
"Roskilde","Djursland","Silkeborg") )),size=2) +

\#scale_shape_manual(values $=c(103,99,100,115))+$

theme_bw()+

theme_rgbw ()$+$

theme_rgbw(base_size $=11$,base_family $=$ "'")+

labs(shape="Areas")+

scale_T_continuous $($ limits $=\mathrm{c}(0.0,8))+$

scale_L_continuous $(\text { limits }=\mathrm{c}(0.0,0.8))^{+}$

scale_R_continuous $(\operatorname{limits}=\mathrm{c}(0.2,1))$

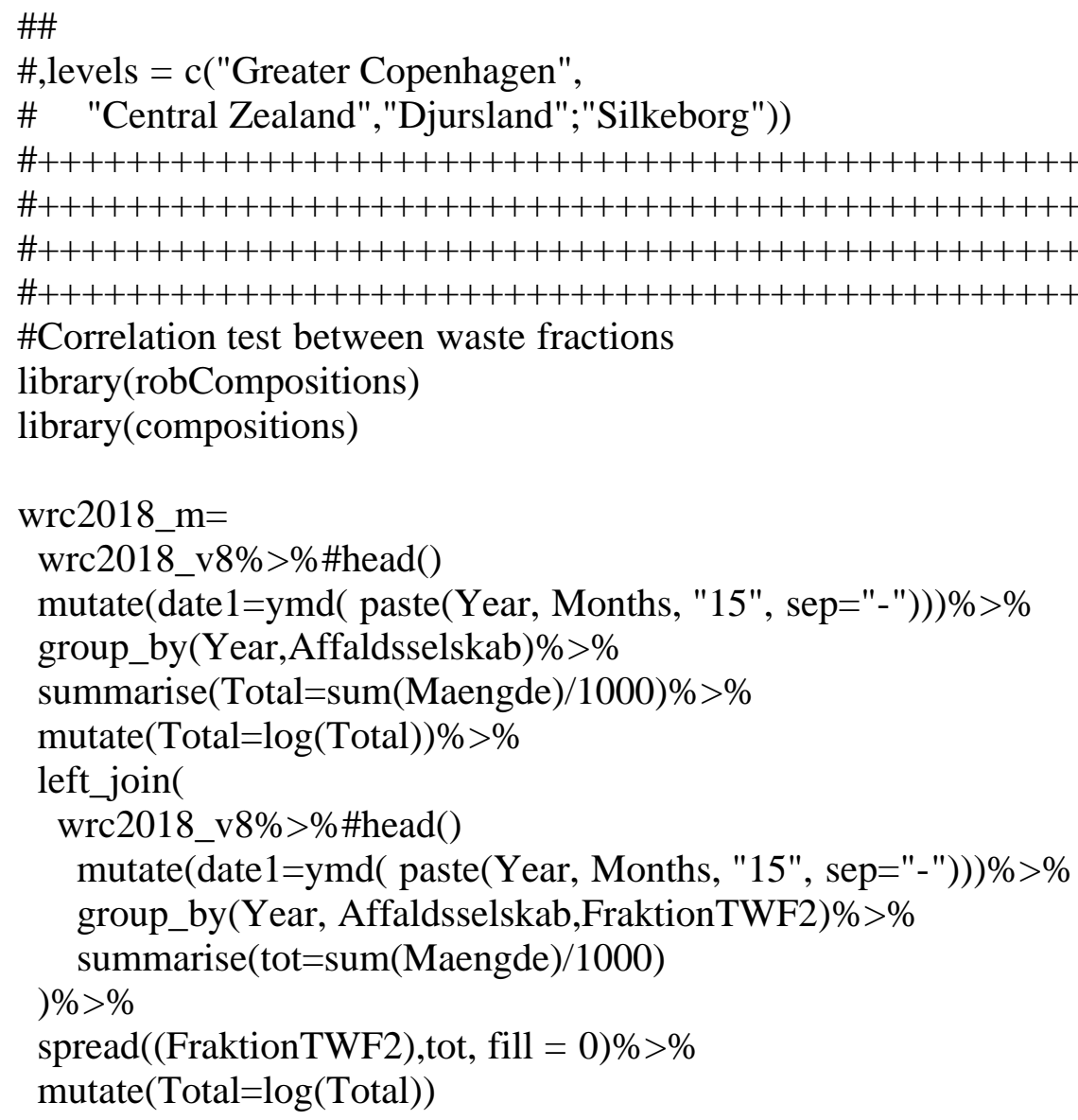




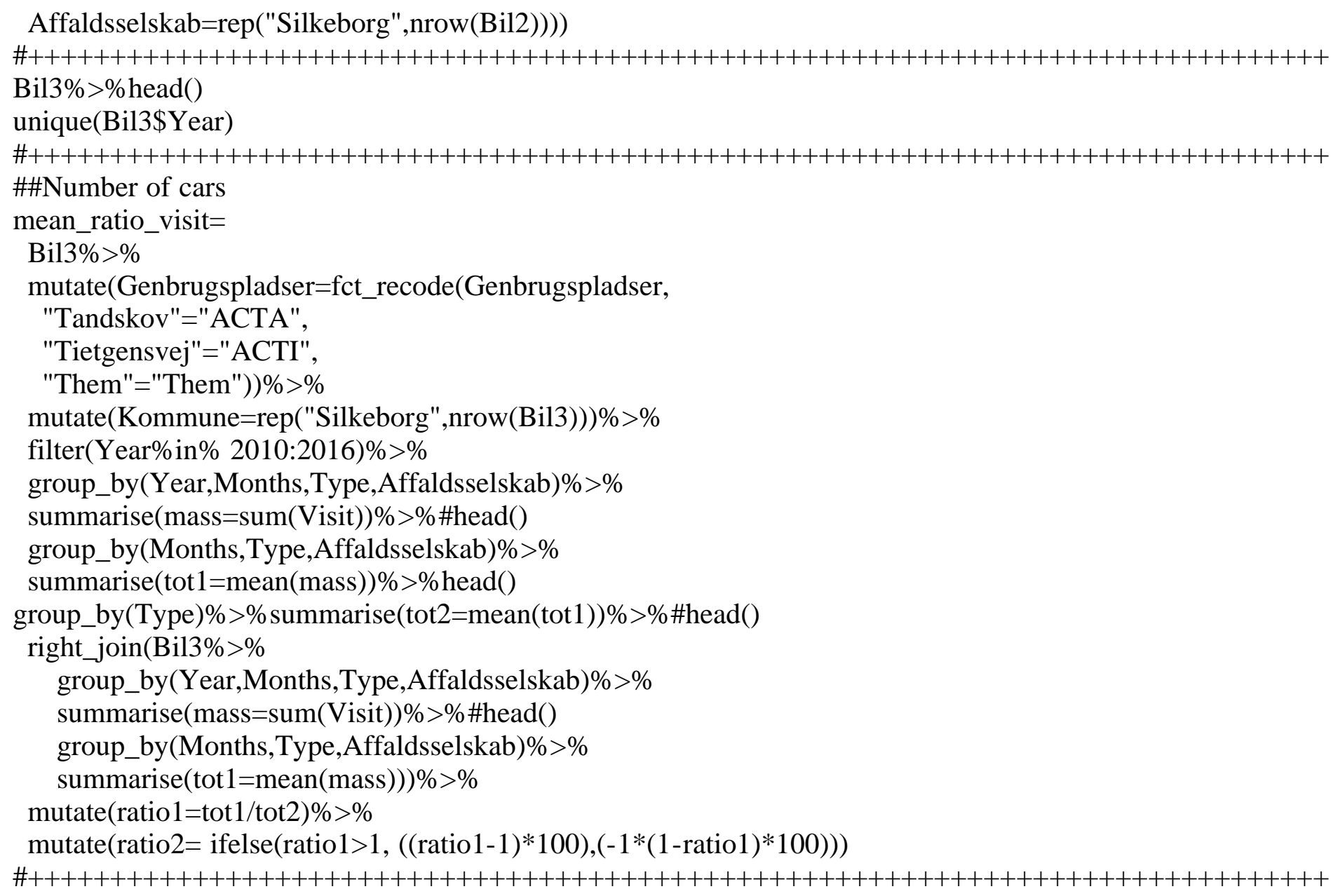




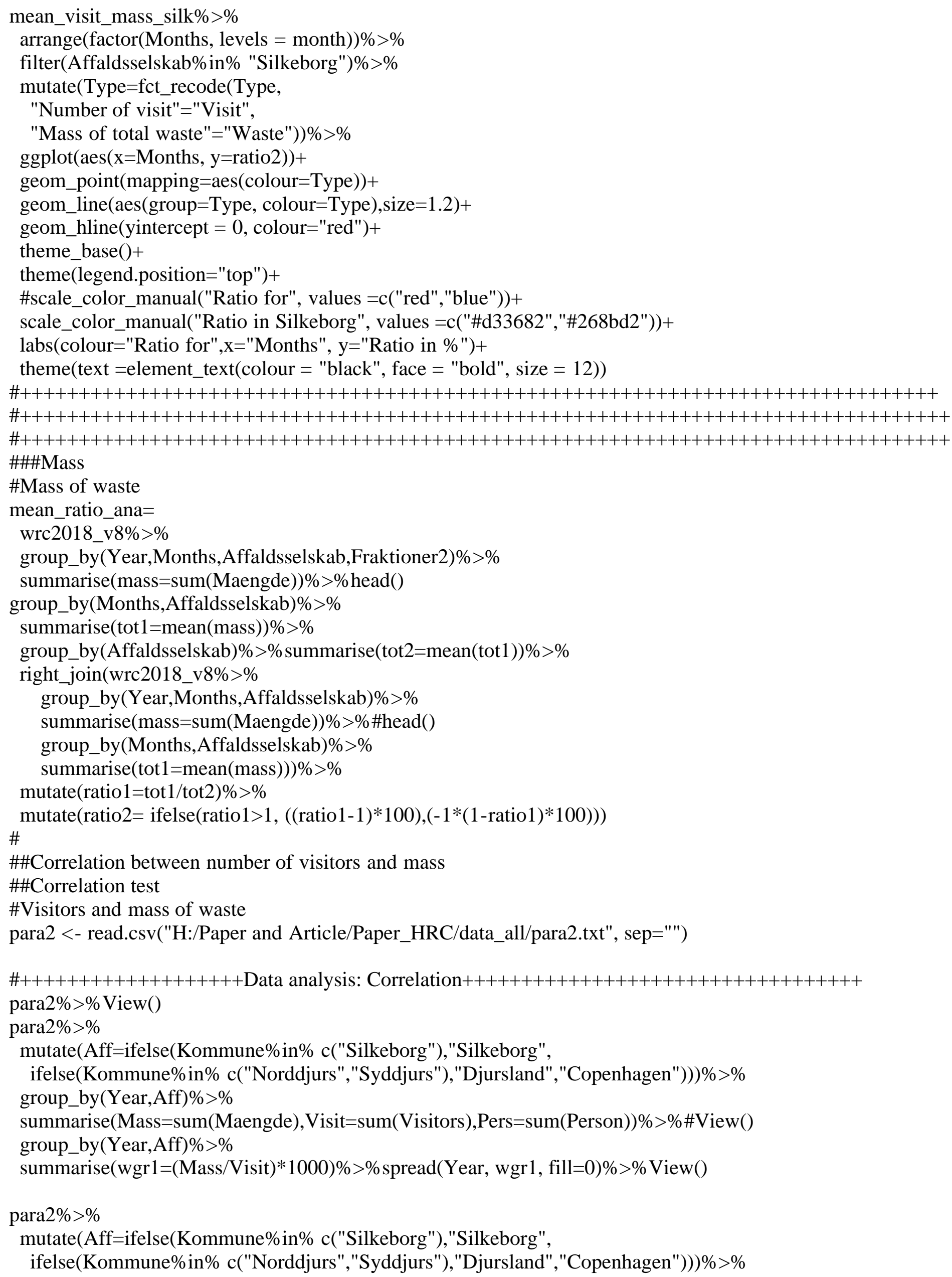


\#\#Waste treatment forms

wrc2018_v8\%>\%

group_by(Year,Months,Affaldsselskab,Treatment)\%>\%

summarise(mass=sum(Maengde)) $\%>\%$

group_by(Months,Affaldsselskab,Treatment)\%>\%

summarise(tot1=median(mass)) $\%>\%$

group_by(Affaldsselskab,Treatment)\%>\%summarise(tot2=median(tot1))\%>\%

right_join(wrc2018_v8\%>\%

group_by(Year,Months,Affaldsselskab,Treatment)\%>\%

summarise(mass=sum(Maengde))\%>\%\#head()

group_by(Months,Affaldsselskab,Treatment)\%>\%

summarise(tot1=median(mass)) $\%>\%$

mutate(ratio1=tot $1 /$ tot 2$) \%>\%$

mutate $($ ratio2= ifelse $($ ratio1 $>1,(($ ratio1-1)*100), $(-1 *(1-$ ratio1 $) * 100))) \%>\%$

ggplot(aes(Months,ratio2, colour=Treatment))+

geom_line(aes(group=Treatment, colour=Treatment),size=1.2)+geom_point()+

facet_wrap $(\sim$ Affaldsselskab, scales $=$ "free")+

geom_hline(yintercept $=0$, colour="red")

\#\#Waste fraction: Fraktioner2

wrc2018_v8\%>\%

group_by(Year,Months,Affaldsselskab,Fraktioner2)\%>\%

summarise(mass=sum(Maengde))\% $>\%$

group_by(Months,Affaldsselskab,Fraktioner2)\%>\%

summarise(tot1=median(mass))\%>\%

group_by(Affaldsselskab,Fraktioner2)\%>\%summarise(tot2=median(tot1))\%>\%

right_join(wrc2018_v8\%>\%

group_by(Year,Months,Affaldsselskab,Fraktioner2)\%>\%

summarise(mass=sum(Maengde))\%>\%\#head()

group_by(Months,Affaldsselskab,Fraktioner2)\%>\%

summarise(tot1=median(mass)) $\%>\%$

mutate(ratio1=tot $1 /$ tot 2$) \%>\%$

mutate $($ ratio2= ifelse $($ ratio1 $>1,(($ ratio1-1)*100), $(-1 *(1-$ ratio1 $) * 100))) \%>\%$

ggplot(aes(Months,ratio2, colour=Fraktioner2))+

geom_line(aes(group=Fraktioner2, colour=Fraktioner2),size=1.2)+geom_point()+

facet_wrap $(\sim$ Affaldsselskab, scales = "free" $)+$

geom_hline(yintercept $=0$, colour="red")

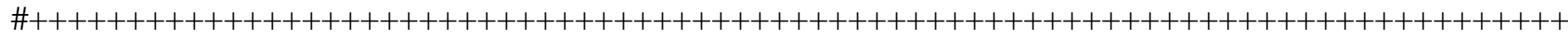

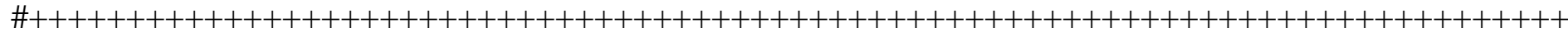

\#data:

library(readxl)

"\%! in\%" <- function(x,table) match(x,table, nomatch =0) == 0

library(tidyverse)

library(lubridate)

library(forcats)

library(forecast)

library(ggfortify)

library(ggseas)

\#+++++

visit_v1 <-read_excel("H:/Paper and Article/Paper_HRC/data_all/visit_v1.xlsx")

visit_v1=visit_v1\%>\% mutate(date1=ymd( paste(Year, Months, "15", sep="-")))\#\%>

visit_v1\$Months=factor(visit_v1\$Months,levels=c("Jan","Feb","Mar","Apr","Maj"

,"Jun","Jul","Aug","Sep","Okt","Nov","Dec")) 
wrc2018_v8= read.table("H:/Paper and Article/Paper_HRC/data_all/wrc2018_v8.txt")

wrc2018_v8=wrc2018_v8\%>\% mutate(date1=ymd( paste(Year, Months, "15", sep="-")))

wrc2018_v8\$Months=factor(wrc2018_v8\$Months,levels=c("Jan","Feb","Mar","Apr","Maj"

,"Jun","Jul","Aug","Sep","Okt","Nov","Dec"))

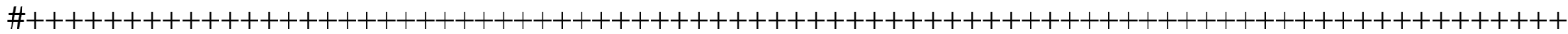

visit_v1\%>\%group_by(Year,Affaldsselskab,Kommune)\%>\%

summarise(Visit=sum(Visit))\%>\%head()

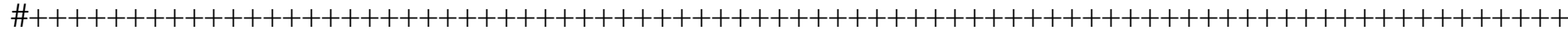

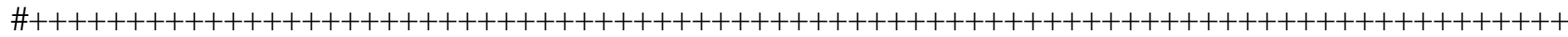

\#Combine waste data and number of visitors

waste_visit $=$ wrc2018_v8\%>\%

mutate(date1=ymd( paste0(Year, Months, "15", sep="-")))\%>\%\#transform to date

group_by(Year,Months,date1,Affaldsselskab)\%>\%

summarise (Total=sum(Maengde) $) \%>\%$

left_join(\#combine two tables

wrc2018_v8\%>\%\#head()

mutate(date1=ymd( paste0(Year, Months, "15", sep="-")))\%>\%\#transform to date

group_by(Year,Months,date1, Affaldsselskab,Fraktioner2)\%>\%

summarise(tot=sum(Maengde) $)$ ) $\%>\%$

left_join(\#combine two tables

visit_v1\%>\%

mutate(Affaldsselskab=fct_recode(Affaldsselskab,

"Roskilde"="Central Zealand"))\%>\%\#rename a row

mutate(date1=ymd( paste0(Year, Months, "15", sep="-")))\%>\%

mutate(Year=as.double(Year),Months=as.factor(Months))\%>\%

group_by(Year,Months,date1,Affaldsselskab)\%>\%

summarise(Visit=sum(Visit)) $\%>\%$

filter(Affaldsselskab\%!in\% c("Copenhagen","Djursland"),

Year\%!in\% c("2010","2011","2018"))\%>\%

spread(Fraktioner2,tot,fill $=0$ )

$\mathrm{Z}+++++++++++++++++++++++++++++++++++++++++++++++++++++++++++++++++++++++++++++++$

\#\#Table 2: Waste generation rates

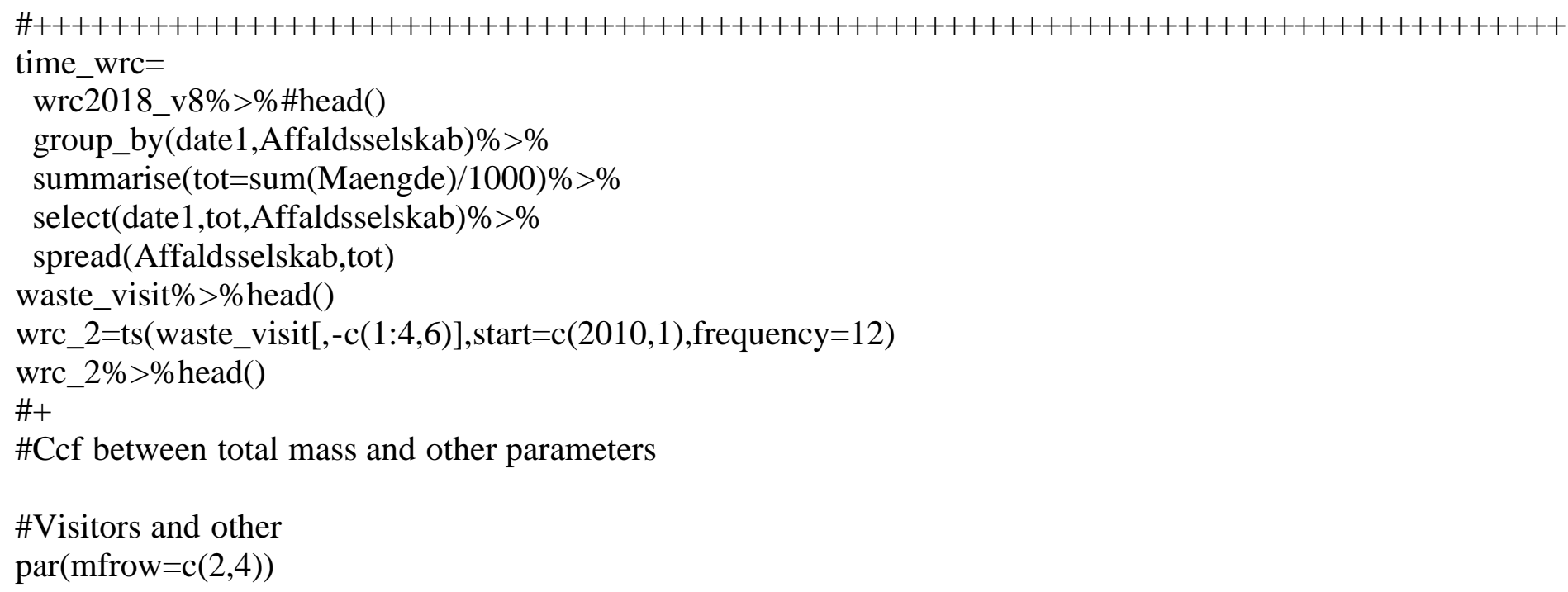




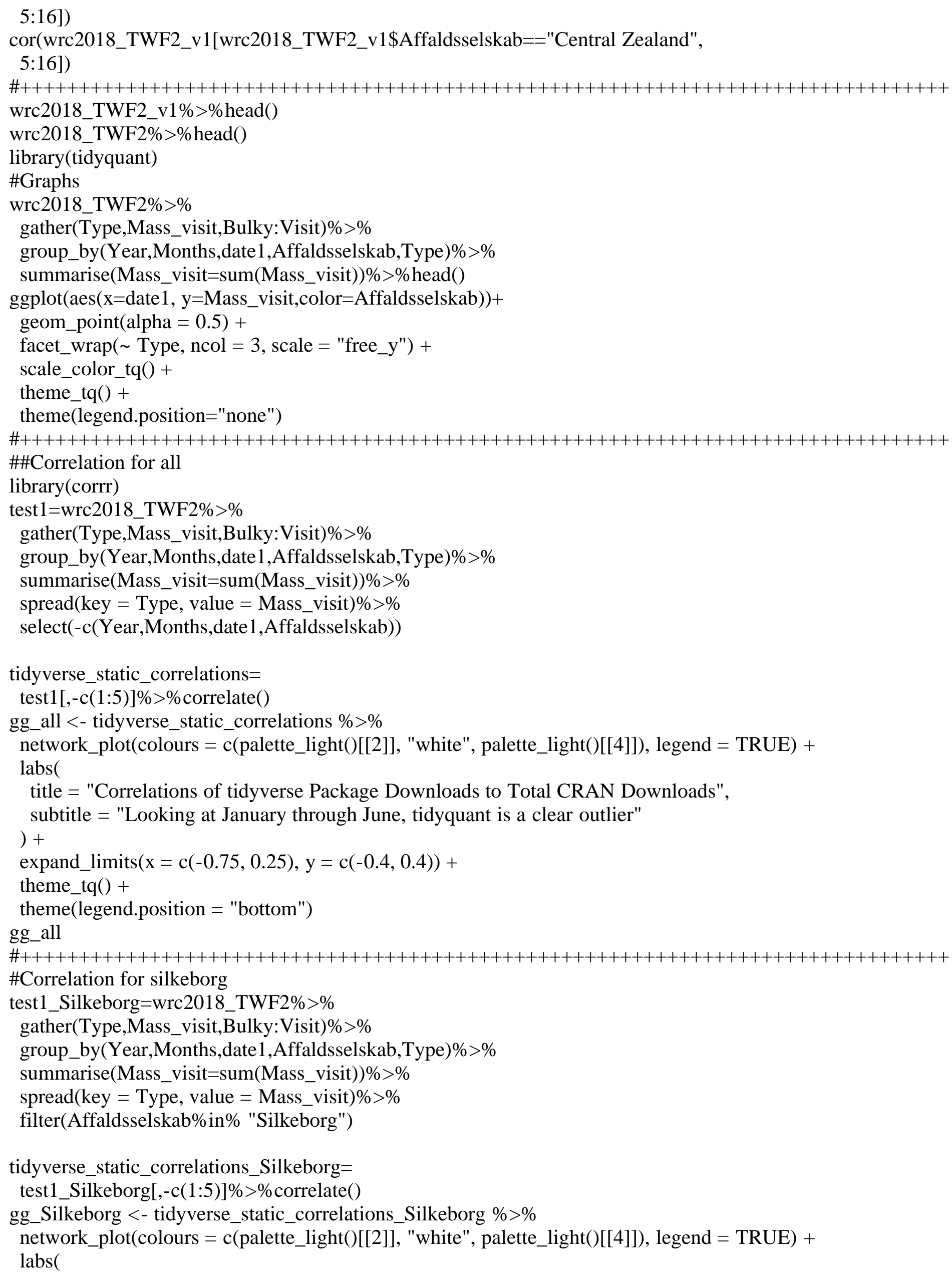


title = "Correlations of tidyverse Package Downloads to Total CRAN Downloads",

subtitle = "Looking at January through June, tidyquant is a clear outlier"

)+

expand_limits $(\mathrm{x}=\mathrm{c}(-0.75,0.25), \mathrm{y}=\mathrm{c}(-0.4,0.4))+$

theme_tq( $)+$

theme(legend.position = "bottom")

gg_Silkeborg

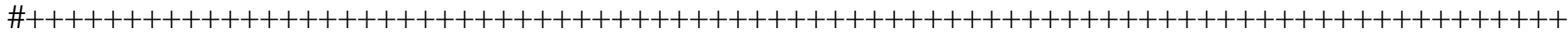

\#Correlation for Central Zealand

test1_Central_Zealand=wrc2018_TWF2\%>\%

gather(Type,Mass_visit,Bulky:Visit)\%>\%

group_by(Year,Months,date1,Affaldsselskab,Type)\%>\%

summarise(Mass_visit=sum(Mass_visit) $\%>\%$

spread $($ key $=$ Type, value $=$ Mass_visit $) \%>\%$

filter(Affaldsselskab\%in\% "Central Zealand")

tidyverse_static_correlations_Central_Zealand=

test1_Central_Zealand[,-c(1:5)]\%>\%correlate()

gg_Central_Zealand <- tidyverse_static_correlations_Central_Zealand \% $>\%$

network_plot(colours = c(palette_light()[[2]], "white", palette_light()[[4]]), legend = TRUE) +

labs(

title = "Correlations of tidyverse Package Downloads to Total CRAN Downloads",

subtitle = "Looking at January through June, tidyquant is a clear outlier"

)+

expand_limits $(\mathrm{x}=\mathrm{c}(-0.75,0.25), \mathrm{y}=\mathrm{c}(-0.4,0.4))+$

theme_tq ()$+$

theme(legend.position = "bottom")

gg_Central_Zealand

\#++++++++++++++++++++++++++++++++++++++++++++++++++++++++++++++++++++++++++++++++

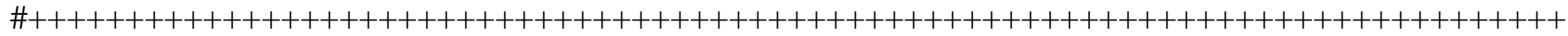

\#+++++++++++++++++++++++++++++++++++++++++++++++++++++++++++++++++++++++++++++++

\#data:

library(readxl)

visit_v1 <-read_excel("H:/Paper and Article/Paper_HRC/data_all/visit_v1.xlsx")

wrc2018_v8= read.table("H:/Paper and Article/Paper_HRC/data_all/wrc2018_v8.txt")

\#Group data number of visitors and mass per waste frations

Visit_all=

visit_v1\%>\%head()

mutate(Year=as.integer(Year))\% $>\%$

mutate(Months=as.factor(Months))\%>\%

mutate(Affaldsselskab=as.factor(Affaldsselskab))\%>\%

mutate(Kommune $=$ as.factor $($ Kommune $) \%>\%$

select(-c(Type))\%>\%

group_by(Year,Months,Affaldsselskab)\%>\%

summarise(Visit=sum(Visit))\%>\%

filter(Year\%!in\% c("2017","2018"))

Visit_all\%>\%View()

\#ONly waste for Silkebord and Roskilde from 2012 to 2016 


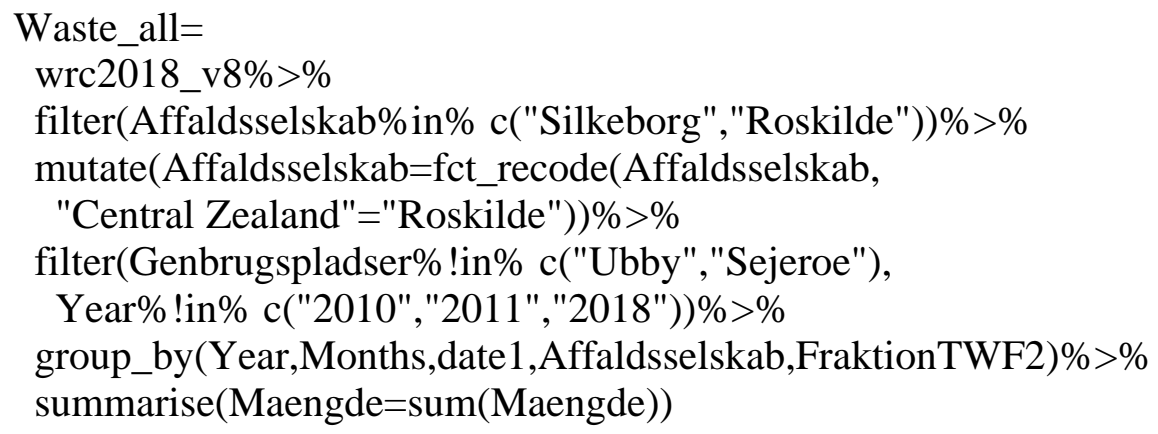




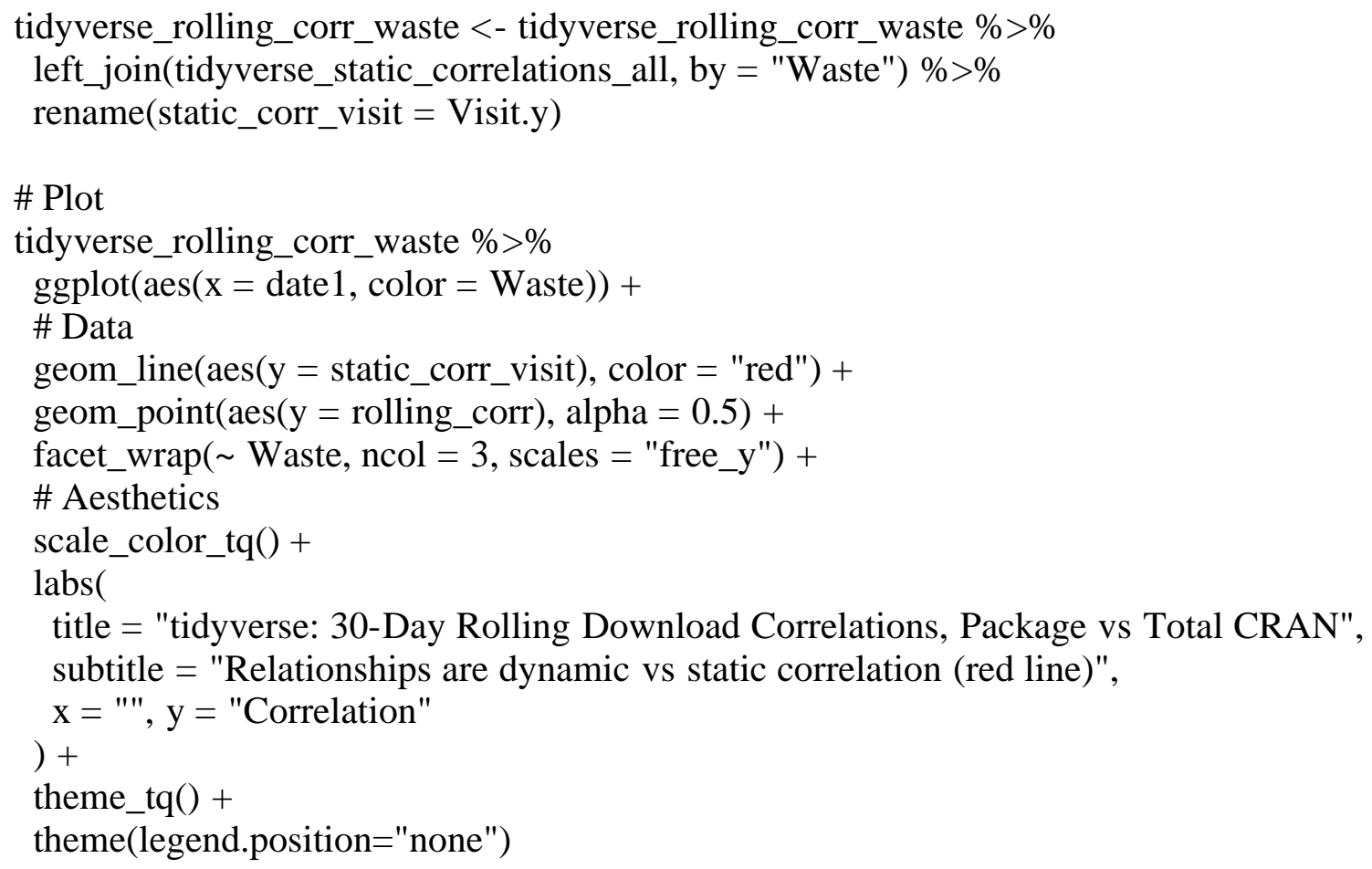


ggplot(aes(x=factor(Treatment,levels = c("Recycling","Incineration","Other")),

$\mathrm{y}=$ ratio,fill=factor(Months)) $)+$

geom_bar(position="dodge", stat="identity")+

geom_text(aes(x=factor(Treatment,levels = c("Recycling","Incineration","Other")),

$\mathrm{y}=$ ratio, $\mathrm{ymax}=$ ratio,

label $=$ procent,procent $=$ procent +.05 ,

\#hjust=ifelse(sign(procent) $>0,1,0$ )

vjust $=0$ ),

colour="black",position =position_dodge $($ width $=0.85))+$

labs(colour="År")+ylab("Ratio")+xlab("Waste fraction")+

facet_wrap $(\sim$ factor(Affaldsselskab,levels $=$ selk $))+\#$ +\#cales = "free"

theme_bw( $)+$

theme(legend.text=element_text(size $=12))^{+}$

theme(legend.position="top")+

scale_fill_manual("Months", values = yearcol)+

guides(title="Months", fill = guide_legend $($ nrow $=1))+$

ylab("Ratio")+xlab("Affaldsfraktioner")+

theme $($ text $=$ element_text $($ colour $=$ "black", face $=$ "bold", size $=14))+$

theme $($ axis.text $=$ element_text $($ color $=$ "black", size $=12))+$

theme(legend.text=element_text(size $=12))^{+}$

ylab("Log-ratio")+

xlab("Waste fractions")

ggsave("geometric_treat.wmf",width $=20$, height $=20$, units $=$ "cm")

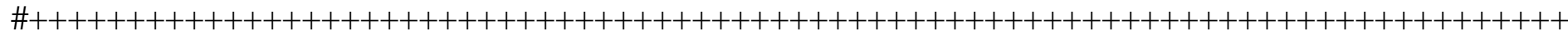

seascomp $=$

wrc2018_v8\%>\%

group_by(Year,Months,Affaldsselskab,Kommune,Genbrugspladser,FraktionTWF2)\%>\%

summarise(tot=sum(Maengde) $) \% \%$

spread(FraktionTWF2,tot)

library(writexl)

write_xlsx(seascomp, "H:/Paper and Article/Paper_HRC/Results/2018-05-Results/seascomp.xlsx")

seascomp_djur=seascomp\%>\%filter(Affaldsselskab\%in\%"Djursland" )

anova(Im(ilr((seascomp_djur[,5:15])) Months, data=seascomp_djur))

seascomp $\%>\%$ head()

\#++++++++++++

library(compositions)

fitt $=\operatorname{lm}(\mathrm{il}(($ seascomp[,5:15])) Months, data=seascomp)

anova $(\operatorname{lm}(\mathrm{i} \operatorname{li}(($ seascomp[,5:15])) Months, data=seascomp $))$

anova(lm(ilr((seascomp[seascomp\$Affaldsselskab=="Djursland",5:15])) Months, data=seascomp))

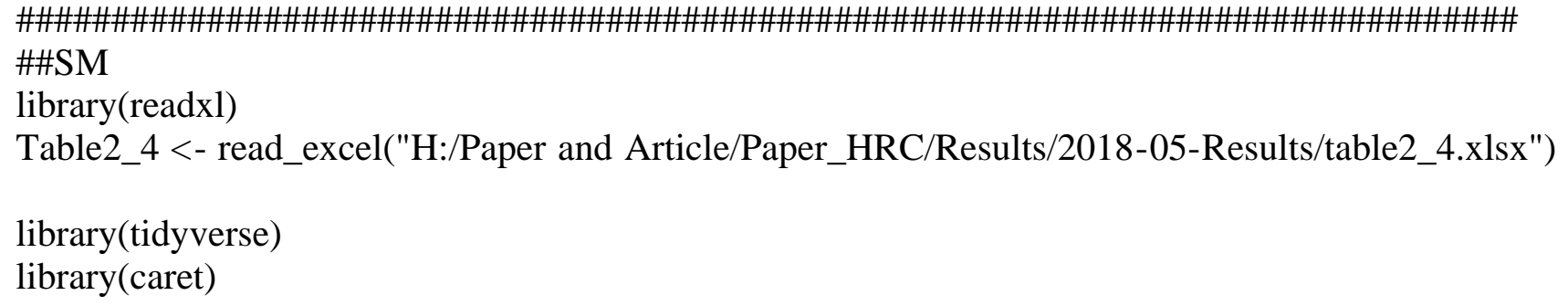


library(corrplot)

library(corrr)

library(broom)

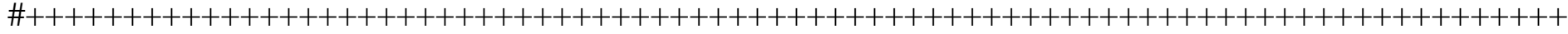

SMTable2=

Table2_4\%>\%

mutate(Visi_house=Visitors/Household,Visi_pers=Visitors/Person)\% $\%$ \%

select(Year,Affaldsselskab,Kommune,Visi_house,Visi_pers)\%>\%

gather(Vaa, vaaa, c(Visi_house,Visi_pers))\%>\%spread(Year,vaaa)\%>\%

mutate(Affaldsselskab=factor(Affaldsselskab,

levels = c("Greater Copenhagen","Central Zealand","Djursland","Silkeborg")))\%>\%

mutate(Kommune=factor(Kommune,levels = c("Frederiksberg","Dragoer","Hvidovre",

"Koebenhavn","Taarnby","Koege","Kalundborg","Lejre",

"Stevns","Roskilde","Syddjurs","Norddjurs","Silkeborg")))\%>\%

arrange(Affaldsselskab,Kommune)\%>\%replace(., is.na(.), "-")\%>\%

mutate_if(is.numeric, funs(round(., 2)))

SMTable2\%>\%View()

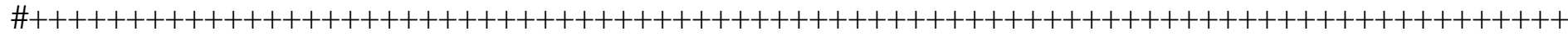

\#\#Population split into single and multi family

DK_all <-read_excel("H:/Paper and Article/Paper_HRC/data_all/DK_all.xlsx",

sheet $=$ "Sheet1")

DK_2010_2017=

DK_all\%>\%

mutate(Kommune=str_replace_all(Kommune,

c("æ"="аe","å"="aа","ø"="oe","Аี"="Aa","Ø"="Oe","Æ"="Ae")))\%>\%\#change danish

\#letters to english

mutate(Pers=fct_recode(Pers,

"7 personer"="7 personer og derover"))\%>\%\#recode and change some rows

separate(Pers, c("Numb_pers","Just_pers"),

sep $=$ " ", remove $=$ FALSE, convert $=$ FALSE $) \%>\% \#$ separate a column

mutate(Numb_pers=as.double(Numb_pers))\%>\%

mutate(housing_type=ifelse(Housing\%in\% c("Etageboliger"),"MF","SF"))\%>\%

select(-House,-Person,-Just_pers) $\%>\%$

gather(Year,Tot, '2010`:2017`)\%>\%

mutate(Number_pers=Numb_pers*Tot)\%>\%

group_by(Kommune,housing_type,Year)\%>\%

summarise(Household=sum(Tot),Person=sum(Number_pers)) $\%>\%$

gather(Hus_pers,antal,Household:Person) $\%>\%$

unite(Housing,housing_type,Hus_pers,sep = "_")\%>\%

spread(Housing,antal)

SMTable3=

DK_2010_2017\%>\%

mutate(Household=MF_Household+SF_Household)\% $\% \%$

mutate(Person=MF_Person+SF_Person) $\%>\%$

mutate(House_pers=Person/Household,

House_pers_SF=SF_Person/SF_Household,

House_pers_MF=MF_Person/MF_Household)\% $\% \%$

select(Year,Kommune,House_pers,House_pers_SF,House_pers_MF)\% $\% \%$

gather(Vaa, vaaa, c(House_pers,House_pers_SF,House_pers_MF))\% $>\%$

spread(Year,vaaa)\%>\% 
filter(Kommune\%in\%c("Frederiksberg","Dragoer","Hvidovre",

"Koebenhavn","Taarnby","Koege","Kalundborg","Lejre",

"Stevns","Roskilde","Syddjurs","Norddjurs","Silkeborg"))\%>\%

ungroup ()$\%>\%$

mutate(Kommune=factor(Kommune,levels = c("Frederiksberg","Dragoer","Hvidovre",

"Koebenhavn","Taarnby","Koege","Kalundborg","Lejre",

"Stevns","Roskilde","Syddjurs","Norddjurs","Silkeborg")))\%>\%

arrange(Kommune)\% $\% \%$

\#replace(., is.na(.), "-")\%>\%

mutate_if(is.numeric, funs(round(., 2)))

SMTable3\%>\%View()

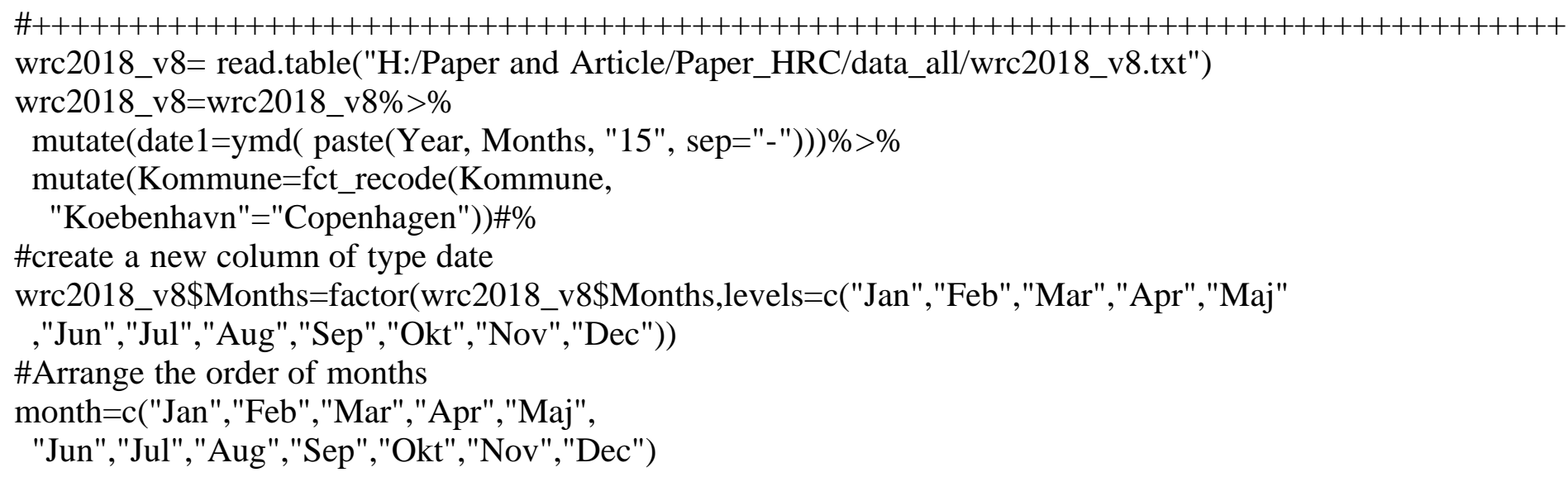


arrange(Affaldsselskab,Kommune,FraktionTWF2)\%>\%

mutate_if(is.numeric, funs(round(., 2)))

SMTable11\%>\%View()

\#Export Table 3

library(writexl)

write_xlsx(SMTable11, "H:/Paper and Article/Paper_HRC/Results/2018-05-Results/SMTable11.xlsx")

\#SM Table 11

\#\#

Table2_4\%>\%

select( Visitors, area_km2,Household, Person, MF_Household, MF_Person, SF_Household, SF_Person)\%>\% correlate() $\%>\%$ focus(Visitors)

\#+++++++++++++++++++++++++++++++++++++++++++++++++++++++++++++++++++++++++++++++

Table2_4\%>\%

group_by(Affaldsselskab)\% $>\%$

\#select( Visitors, area_km2,Household, Person, MF_Household, MF_Person, SF_Household, SF_Person)\% $\%$ \% do(tidy(cor(.\$Visitors,.\$Household)))

\section{.\$Person,}

.\$MF_Household,.\$MF_Person,.\$SF_Household,.\$SF_Person

\#+++++++++++++++++++++++++++++++++++++++++++++++++++++++++++++++++++++++++++++++ regressions $<-$ Table2_4\%>\% group_by(Affaldsselskab) $\%>\%$

do(fit $=\operatorname{lm}($ Visitors $\sim$ area_km2+Household + Person+ MF_Household+MF_Person+ SF_Household+ SF_Person, .)) regressions

regressions $\%>\%$ tidy(fit)\% $\% \% \operatorname{View}()$

regressions $\%>\%$ glance(fit)

\#+++++++++++++++++++++++++++++++++++++++++++++++++++++++++++++++++++++++++++++++

regressions $1<-$ Table2_4 \%>\% group_by(Affaldsselskab) \%>\%

do(fit1 = $\operatorname{lm}(\log ($ Visitors $)$ area_km2+log(Household) $+\log ($ Person $)+\log ($ MF_Household)+log(MF_Person) + regressions1 $\log ($ SF_Household $)+\log ($ SF_Person $),)$.

regressions $1 \%>\%$ tidy(fit1)\%>\%View()

regressions $\%>\%$ glance(fit)

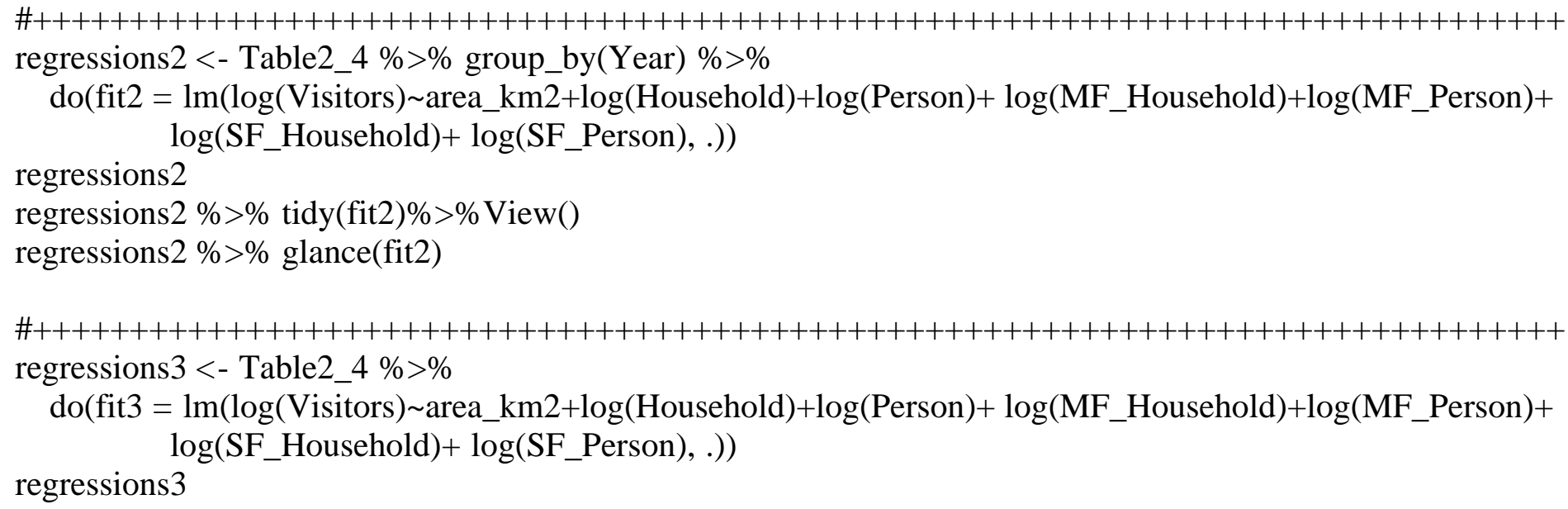


regressions3 \%>\% tidy(fit3)\%>\%View()

regressions $3 \%>\%$ glance(fit3)

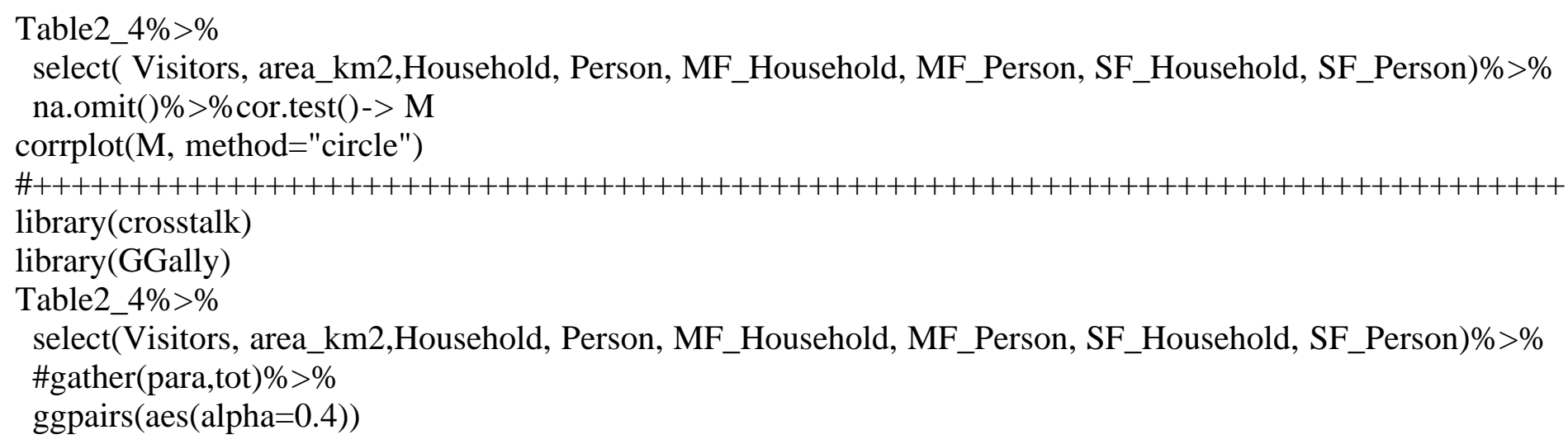


rf1 <- train(Visitors ., data = Table_test, method = "rf")

class $(\operatorname{lm} 1)$

attributes $(\operatorname{lm} 1)$

$\operatorname{lm} 1$ \$finalModel

rf1\$finalModel

summary(lm1\$finalModel)\$r.squared

$\operatorname{lm} 1$ \$results

rf1\$results

fitted <- predict $(\operatorname{lm} 1)$

model_mars $=$ train(Visitors ., data=Table_test, method='earth')

plot(model_mars, main="Model Accuracies with MARS")

varimp_mars <- varImp(model_mars)

plot(varimp_mars, main="Variable Importance with MARS")

\#+++++++++

samp <- createDataPartition(Table_test\$Visitors, $\mathrm{p}=0.8$, list $=$ FALSE)

training $<-$ Table_test[samp,]

testing $<-$ Table_test[-samp,]

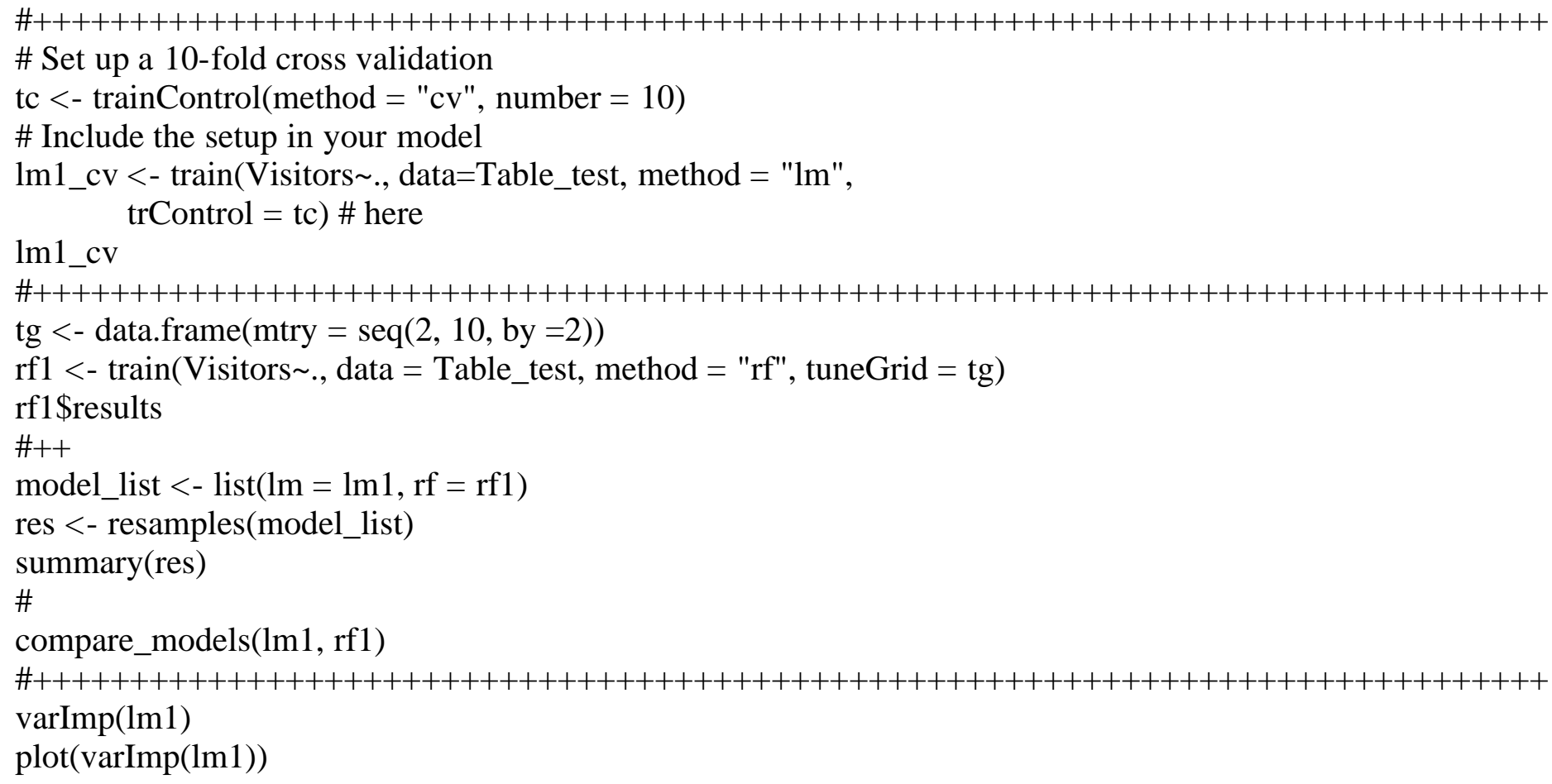

